\title{
THE STAPL PARALLEL CONTAINER FRAMEWORK
}

\author{
A Dissertation \\ by \\ ILIE GABRIEL TANASE
}

\begin{abstract}
Submitted to the Office of Graduate Studies of Texas A\&M University in partial fulfillment of the requirements for the degree of

DOCTOR OF PHILOSOPHY
\end{abstract}

December 2010

Major Subject: Computer Science 


\title{
THE STAPL PARALLEL CONTAINER FRAMEWORK
}

\author{
A Dissertation \\ by \\ ILIE GABRIEL TANASE
}

\begin{abstract}
Submitted to the Office of Graduate Studies of
Texas A\&M University

in partial fulfillment of the requirements for the degree of

DOCTOR OF PHILOSOPHY
\end{abstract}

\begin{abstract}
Approved by:
Co-Chairs of Committee, Lawrence Rauchwerger

Nancy M. Amato

Committee Members, Jennifer Welch

Marvin Adams

Head of Department, Valerie E. Taylor
\end{abstract}

December 2010

Major Subject: Computer Science 


\begin{abstract}
The STAPL Parallel Container Framework. (December 2010)

Ilie Gabriel Tanase, B.S., Polytechnic University Bucharest, Romania;

M.S., Polytechnic University of Bucharest, Romania

Co-Chairs of Advisory Committee: Dr. Lawrence Rauchwerger

Dr. Nancy M. Amato
\end{abstract}

The Standard Template Adaptive Parallel Library (STAPL) is a parallel programming infrastructure that extends $\mathrm{C}++$ with support for parallelism. STAPL provides a run-time system, a collection of distributed data structures (pContainers) and parallel algorithms (pAlgorithms), and a generic methodology for extending them to provide customized functionality.

Parallel containers are data structures addressing issues related to data partitioning, distribution, communication, synchronization, load balancing, and thread safety. This dissertation presents the STAPL Parallel Container Framework (PCF), which is designed to facilitate the development of generic parallel containers. We introduce a set of concepts and a methodology for assembling a pContainer from existing sequential or parallel containers without requiring the programmer to deal with concurrency or data distribution issues. The STAPL PCF provides a large number of basic data parallel structures (e.g., pArray, pList, pVector, pMatrix, pGraph, pMap, pSet). The STAPL PCF is distinguished from existing work by offering a class hierarchy and a composition mechanism which allows users to extend and customize the current container base for improved application expressivity and performance.

We evaluate the performance of the STAPL pContainers on various parallel machines including a massively parallel CRAY XT4 system and an IBM P5-575 cluster. We show that the pContainer methods, generic pAlgorithms, and different applica- 
tions, all provide good scalability on more than $10^{4}$ processors. 
To my wife, Aniela who is always by my side

To my parents 


\section{ACKNOWLEDGMENTS}

First of all I would like to thank my advisors, Dr. Lawrence Rauchwerger and Dr. Nancy Amato, for their continuous support and encouragement throughout my $\mathrm{PhD}$ studies. It has been a long and difficult road with numerous challenges. However together we managed to overcome them no matter how difficult they seemed to be initially. Throughout the years I have learned from them the importance of the high level discussions on why is our research relevant, what distinguishes us from previous and other similar projects and what is the general direction we want to stir our project. As a fresh graduate student I remember being always eager to get things done as soon as possible. From them I have learned that the implementation is not necessarily the most important part. A careful design and documentation that can be discussed and argued for, is more important than the implementation itself. The skills they thought me helped me throughout my PhD studies and hopefully I passed some of this knowledge to the undergraduate and graduate students that I mentored.

I would like to thank my committee members, Dr. Marvin Adams and Dr. Jennifer Welch, who have been of great help with both their comments and creative ideas. With Dr. Adams I interacted while developing a large scale parallel transport application that involved a large number of people from different area of expertise like Computer Science, Nuclear Engineering and Math. I learned from him the importance of providing the right layers of abstractions so that Nuclear Engineering students not familiar with parallel programming can still write efficient programs. From Dr. Jennifer Welch I learned the importance of formally specifying a distributed system behavior so that there is no ambiguity for users when interacting with such a system.

Throughout the graduate school I had the opportunity to interact with a large number of colleagues that all have contributed in one way or another to my education 
as a scientist. First I would like to thank all my STAPL colleagues: Alin Jula, Ping An, Paul Thomas, Silvius Rus, Chidambareswaran Raman, Tao Huang, Paul Thomas, Steven Saunders, William McLendon, Lidia Smith, Timmie Smith, Nathan Thomas, Xiabing Xu, Antal Buss, Adam Fidel, Ioannis Papadopoulos, Shuai Ye, Harshvardhan, Mani Zandifar, Jeremy Vu, Olga Pearce, Tarun Jain, Shishir Sharma. For Mauro Bianco, postdoc in our group, special thanks as he carefully read my design documents and help me clarify the aspects that were not well explained. Special thanks to all my colleagues that were next to me during the long nights we spent in the office while working on papers before deadlines. I will never forget those times.

As part of a large research group I also had the opportunity to interact with students in various areas of research. By getting involved with their work on motion planning, protein folding and particle transport, and listening to their needs in terms of data structure support I greatly improved the usability of my work as presented in this thesis. I would like to thank Shawna Thomas, Lydia Tapia, Sam Ade Jacobs, Roger Pearce, Jae Chang, Teresa Bailey and Alex Maslowski. Also I would like to thank you Anna Tikhonova, Jessie Berlin and Anthony Nowak, undergraduate students that I had the opportunity to mentor throughout the years.

For my late master thesis advisor, Irina Athanasiu, I have only words of gratitude. She first introduced me to the world of research and gave me the opportunity to teach as a teaching assistant at the Polytechnic University of Bucharest, Romania. As a fresh graduate student I still remember the emotions I had while teaching colleagues that were only one year younger than me. Irina first mentioned to me that I can enroll in a PhD program abroad and she recommended me Texas A\&M University and Dr. Lawrence Rauchwerger. Thank you, Irina!

Finally a big thank you to all my family for their help and support. Aniela, my wife, has been always by my side supporting me in the difficult times and sharing 
with me the numerous happy moments we had throughout graduate school. Thank you to my children, David and Daria, for their always joyful mood. They were always the highlight of my day when coming home tired from school after long debugging sessions. Thank you to my father and my late mother for their constant guidance during my first years of school. I am grateful to them for helping me realize at a very young age the importance and the joy of learning. I am trying to pass on to my kids the values they taught me throughout the years. 


\section{TABLE OF CONTENTS}

CHAPTER

INTRODUCTION . . . . . . . . . . . . . . . . . . . . 1

A. Outline . . . . . . . . . . . . . . . 3

II RELATED WORK $\ldots \ldots \ldots \ldots \ldots \ldots \ldots$

III STAPL OVERVIEW $\ldots \ldots \ldots \ldots \ldots \ldots \ldots$

A. STAPL pView Concept . . . . . . . . . . . . . 13

B. Runtime System . . . . . . . . . . . . . . . . . . 18

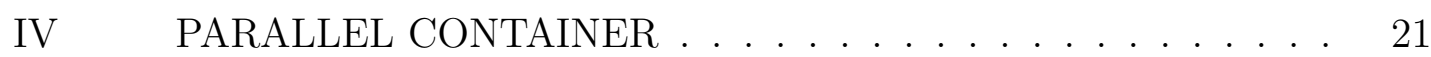

A. pContainer Requirements . . . . . . . . . . . . . . 21

B. pContainer Definition . . . . . . . . . . . . . . 23

1. Set Theory Definitions . . . . . . . . . . . . . 24

2. pContainer Domain . . . . . . . . . . 26

3. Ordered Domain . . . . . . . . . . . . . 26

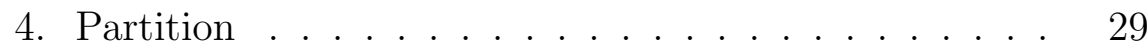

5. Ordered Partition of Total Ordered Domains . . . . 30

C. pContainer Composability . . . . . . . . . . . . . 32

V PARALLEL CONTAINER FRAMEWORK $\ldots \ldots \ldots \ldots$

A. pContainer Framework Design . . . . . . . . . . 38

B. pContainer Interfaces . . . . . . . . . . . . . . . . 39

C. Shared Object View Implementation _. . . . . . . . . . 42

1. Base Container Interface . . . . . . . . . . . . 46

2. Location Manager Interface . . . . . . . . . . . 48

3. Domain Interface . . . . . . . . . . . . . . . 49

4. Partition Interface . . . . . . . . . . . . . 51

5. Partition Mapper Interface . . . . . . . . . . . . 54

6. Data Distribution Manager . . . . . . . . . . . 55

D. Specification for pContainer Framework Concepts . . . . 59

1. pContainer Base . . . . . . . . . . . . . . . 59

2. Static pContainer . . . . . . . . . . . 62

3. Dynamic pContainer . . . . . . . . . . . . . . . 63

4. Indexed pContainer . . . . . . . . . . . . 63 
CHAPTER $\quad$ Page

5. Associative pContainer ......... . . . 67

6. Relational pContainer . . . . . . . . . . . . 68

7. Sequence pContainer . . . . . . . . . . . . 70

E. Integrating all Concepts using pArray Example . . . . . . 71

F. pContainers Implemented in the Framework . . . . . . . . 74

G. pContainer Support for Redistribution . . . . . . . . . 77

1. Data Marshaling . . . . . . . . . . . . . 79

H. pContainer Customization using Traits . . . . . . . . . 80

VI THREAD SAFETY . . . . . . . . . . . . . 83

A. pContainer Thread Safety Design . . . . . . . . . . . 84

B. Data Distribution Manager . . . . . . . . . . . . 85

C. Thread Safety Manager . . . . . . . . . . . . 86

D. Partition Locking Specification . . . . . . . . . . . . . 88

E. pArray and pMatrix .............. . . 89

F. pList . . . . . . . . . . . . . . . . 90

G. Associative pContainers . . . . . . . . . . . . 90

H. pGraph ..................... . . 91

VII MEMORY CONSISTENCY MODEL . . . . . . . . . . . . . . 92

A. pContainer Interfaces . . . . . . . . . . . . . . . . 93

B. Completion Guarantees . . . . . . . . . . . . . . . 95

C. Memory Consistency Conditions . . . . . . . . . . . 98

1. pContainer Default Memory Consistency Model . . . . 98

D. Memory Consistency Example . . . . . . . . . . . . . . 102

E. Other Memory Consistency Models . . . . . . . . . . . . . 103

1. The Default pContainer MCM is not Sequentially Consistent . . . . . . . . . . . . 103

2. The Default pContainer MCM is not Processor Consistent . . . . . . . . . . . . 105

3. Modifying the Default pContainer MCM . . . . . . . . 105

F. pContainer Method: Developer Side . . . . . . . . . . 107

G. Consistency of Other pContainer Methods . . . . . . . . . 110

H. Enforcing Synchronization Points Automatically . . . . . . 111

VIII PCONTAINER PERFORMANCE EVALUATION . . . . . . . 113

A. Experimental Setup . . . . . . . . . . . . . . . . 113

B. Evaluation of pContainer Methods . . . . . . . . . . 114 
C. Evaluation of Generic Algorithms . . . . . . . . . . . . 115

D. Specific Applications . . . . . . . . . . . . . 115

IX THE STAPL PARRAY . . . . . . . . . . . . 116

A. Example . . . . . . . . . . . . . . . 117

B. The pArray Specification . . . . . . . . . . . . . . 118

C. pArray Partitions . . . . . . . . . . . . . . . . . . 119

D. pArray Customization . . . . . . . . . . . . 120

E. Performance Evaluation . . . . . . . . . . . . . 121

1. Methods ................. . . 122

2. Algorithms . . . . . . . . . . . . . . . 128

F. Memory Consumption Study . . . . . . . . . . . . . 130

X THE STAPL PLIST . . . . . . . . . . . . 133

A. pList Example . . . . . . . . . . . . . . . . . . 134

B. pList Specification . . . . . . . . . . . . . . . 134

C. pList Design and Implementation . . . . . . . . . . . . 136

D. Performance Evaluation . . . . . . . . . . . . . . 140

E. pList Method Evaluation . . . . . . . . . . . . . . . . . . . 141

F. pAlgorithm Comparison . . . . . . . . . . . . . . . . . . 142

G. Comparison of Dynamic Data Structures in STAPL . . . . 144

H. Application using pList: Euler Tour . . . . . . . . . . . 145

XI THE STAPL PGRAPH . . . . . . . . . . . . . . . . . 149

A. pGraph Example . . . . . . . . . . . . . . . . 150

B. pGraph Concepts and Interfaces . . . . . . . . . . . . . 151

C. pGraph Class Hierarchy . . . . . . . . . . . . . . . . 153

D. pGraph Design and Implementation . . . . . . . . . . . . . 157

E. pGraph pViews . . . . . . . . . . . . . . . . . 159

F. Performance Evaluation . . . . . . . . . . . . . . . . 161

1. pGraph Methods Evaluation . . . . . . . . . . 161

2. Evaluation of Address Translation Mechanisms . . . . 164

3. pGraph Algorithms . . . . . . . . . . . . . . 167

4. Page Rank . . . . . . . . . . . . . . . . . . . . . 172

XII ASSOCIATIVE PCONTAINERS . . . . . . . . . . . 173

A. Associative pContainer Specification . . . . . . . . . . 174

B. Associative pContainer Design and Implementation . . . . 176 
CHAPTER

C. Performance Evaluation . . . . . . . . . . . . . . . . 179

1. MapReduce . . . . . . . . . . . . . . . . 179

2. Generic Algorithms . . . . . . . . . . . . 180

XIII PCONTAINER COMPOSITION EVALUATION . . . . . . 182

XIV CONCLUSION AND FUTURE WORK . . . . . . . . . 186

REFERENCES . . . . . . . . . . . . . . . . . . . . . . . . . 189

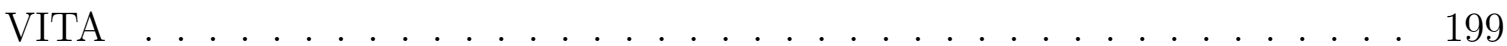




\section{LIST OF TABLES}

TABLE

I Comparison with related projects. . . . . . . . . . . . 8

II STAPL pViews and corresponding operations. . . . . . . . . . . . . 16

III Base container interface. . . . . . . . . . . . . . . . 47

IV Location manager interface. . . . . . . . . . . . . . . . 48

$\mathrm{V} \quad$ Ordered domain interface. . . . . . . . . . . . . 50

VI Finite ordered domain interface. . . . . . . . . . . . . . . 51

VII Partition base interface. . . . . . . . . . . . . . . 52

VIII Ordered partition interface. . . . . . . . . . . . 53

IX Partition mapper interface. . . . . . . . . . . . . . . 54

X Data distribution manager interface. . . . . . . . . 58

XI Base pContainer interface. . . . . . . . . . . . . . 60

XII Static pContainer interface. . . . . . . . . . . . 62

XIII Dynamic pContainer interface. . . . . . . . . . . . 63

XIV Indexed pContainer interface. . . . . . . . . . . . . 64

XV Indexed partition interface. . . . . . . . . . . . 65

XVI Associative pContainer interface. . . . . . . . . . . . 67

XVII Relational pContainer interface. ............. 68

XVIII Sequence pContainer interface. . . . . . . . . . . 70

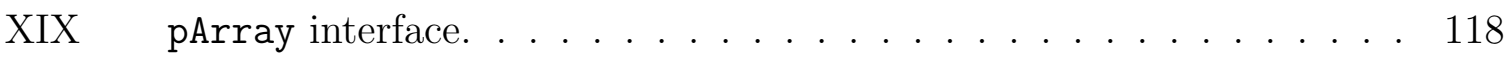

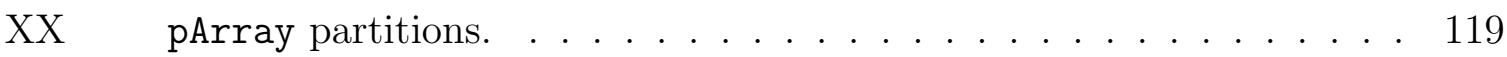


TABLE

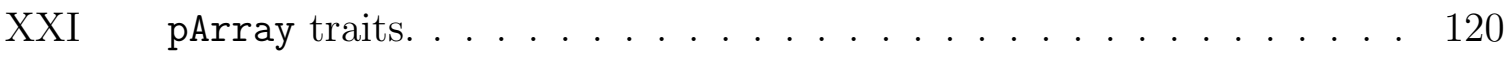

XXII pArray memory consumption. . . . . . . . . . . . . . 130

XXIII Theoretical memory usage for pArray. . . . . . . . . . . . 131

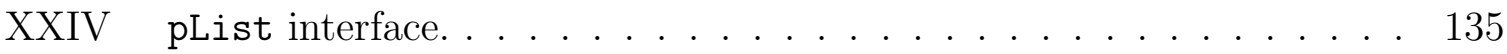

XXV Vertex reference interface. . . . . . . . . . . . . . . 152

XXVI Edge reference interface. . . . . . . . . . . . . . . . 153

XXVII pGraph interface. . . . . . . . . . . . . . . . 155

XXVIII Associative pContainers interface. . . . . . . . . . . . 175 


\section{LIST OF FIGURES}

FIGURE

STAPL overview

Overlap pView example. . . . . . . . . . . . . . . . . . 17

Composed pContainers. . . . . . . . . . . . . . . . . 34

The invoke method of the data distribution manager. . . . . . . 56

10 Example of pContainer deployment on two locations. . . . . . . . . 71

11 Pseudocode of pArray set() method. . . . . . . . . 73

12 pContainers inheritance. . . . . . . . . . . . . 74

13 Redistribution for two given partitions. . . . . . . . . . 78

14 Marshaling interfaces. . . . . . . . . . . . . . . 80

15 pContainer template arguments. . . . . . . . . . . . 81

16 pGraph customization. . . . . . . . . . . . . . 82

$17 \quad$ Generic invoke method implementation with locking statements. . . 87

18 User, pContainer, run time system interaction. . . . . . . . . . 93

19 STAPL program execution. . . . . . . . . . . . . 94

20 Completion guarantees. . . . . . . . . . . . . . . . . 97 
21 Asynchronous methods ordering. . . . . . . . . . . . . . 101

22 Relaxed completion order: (a) Operations on different pContainer elements receive their acknowledgments out of order (b) Dekker's mutual exclusion. . . . . . . . . . . . . . . . . . . . . . . . . 104

23 Processor consistency counter example. . . . . . . . . . . 106

24 Kernel used to evaluate the performance of pContainer methods. . . 114

25 Derivation chain for pArray. . . . . . . . . . . 116

26 pArray example. . . . . . . . . . . . . . . . . 117

27 pArray constructor execution time for various input sizes on (a) CRAY4 and (b) P5-CLuster. . . . . . . . . . . . . . . . . . 121

28 CRAY4: pArray local method invocations for various container sizes. 123

29 CRAY4: pArray methods for various input sizes. . . . . . . . . . . 124

30 CRAY4: pArray methods set_element, get_element and split phase get_element. . . . . . . . . . . . . . 125

31 pArray methods for various percentage of remote invocations. . . . . 126

32 CRAY4: pArray local and remote method invocations for various container sizes. . . . . . . . . . . . . . . . . . . 127

33 Execution times for generic algorithms on CRAY4 for a pArray with 20M elements per processor. . . . . . . . . . . . . . . . 129

34 CRAY4: pArray memory usage study. . . . . . . . . . . . . 132

35 Derivation chain for pList. . . . . . . . . . . 133

36 pList example. . . . . . . . . . . . . . . 135

37 Different partitions and mappings for pList. . . . . . . . . 138

38 pList method implementation. . . . . . . . . . . . . 140 
39 Execution times for pList methods. . . . . . . . . . . . . . 141

40 Execution times for p_for_each, p_generate, p_accumulate algorithms on CRAY4 for pArray and pList. . . . . . . . . . . . . . . 142

41 P5-CLUSTER: Weak scaling for p_for_each allocating processes on the same nodes when possible (curve a) or in different nodes (curve b). . . . . . . . . . . . . . . . . . . . 143

42 Comparison pList and pVector dynamic data structures using a mix of 10M operations (read/write/insert/delete). . . . . . . . . . . . 144

43 Weak scaling of Euler Tour algorithm. . . . . . . . . . . . . 147

44 Execution times for Euler Tour and its applications using a tree made by a single binary tree with 500k or $1 \mathrm{M}$ subtrees per processor. 148 pGraph example. . . . . . . . . . . . . . . . . . . . . 150

46 Graph hierarchy of concepts. . . . . . . . . . . . . . 154

$47 \quad$ pGraph pViews example. . . . . . . . . . . . . . . . 159

48 pGraph pViews: (a) pGraph partitioned pView, (b) region_pview, (c) inner_pview and $(\mathrm{d})$ boundary_pview. . . . . . . . . . . . . . . 160

49 CRAY4: Evaluation of static and dynamic pGraph methods while using the SSCA2 graph generator. . . . . . . . . . . . . 162

50 P5-CLUSTER: Evaluation of static and dynamic pGraph methods while using the SSCA2 graph generator. . . . . . . . . . . . 163

$51 \quad$ Find sources in a directed pGraph using static, dynamic with forwarding and dynamic with no forwarding partitions. . . . . . . . 165

52 Comparison of various pGraph partitions. . . . . . . . . . . 166

53 CRAY4: Execution times for different pGraph algorithms. . . . . . 168

54 CRAY4: pGraph algorithms. . . . . . . . . . . . . . 169

55 P5-CLUSTER: Execution times for different pGraph algorithms. . . . 170 
xviii

FIGURE

Page

$56 \quad$ Page rank for two different input meshes: 1500x1500 and 15x150000. 172

57 Associative pContainer: (a) derivation from the framework base classes (b) associative pContainers internal hierarchy. . . . . . . . . 174

58 Value based partition for sorted associative pContainers. . . . . . 177

59 MapReduce used to count the number of occurrences of every word in Simple English Wikipedia website (1.5GB). . . . . . . . . . . 179

60 CRAY4: Scalability for generic algorithms when using associative pContainers. . . . . . . . . . . . . . . . . . . . 181

61 Example of pContainer composition and nested pAlgorithm invocation. . . . . . . . . . . . . . . . . . . . 183

62 Comparison of pArray<pArray<>> $(p a<p a>)$, plist<pArray<>> $($ plist $<p a>$ ) and pMatrix on computing the minimum value for each row of a matrix. . . . . . . . . . . . . . . . . . . . . . . . . . 184 


\section{CHAPTER I}

\section{INTRODUCTION}

Parallel programming is becoming mainstream due to the increased availability of multiprocessor and multicore architectures and the need to solve larger and more complex problems. The Standard Template Adaptive Parallel Library (STAPL) [16, $4,67,5,68,52,63,67,65,64,15,13,66]$ is being developed to help programmers address the difficulties of parallel programming. STAPL is a parallel $\mathrm{C}++$ library with functionality similar to STL, the ISO adopted C++ Standard Template Library [49]. STL is a collection of basic algorithms, containers and iterators that can be used as high-level building blocks for sequential applications. Similar to STL, STAPL provides a collection of parallel algorithms (pAlgorithms), parallel and distributed containers (pContainers) [63, 65, 64, 15, 66], and pViews to abstract the data access in pContainers. STAPL provides the building blocks for writing parallel programs and the mechanisms (glue) to put them together in large programs. An essential building block for such a generic library is its data structures. Sequential libraries such as STL [49], BGL [30], and MTL [28], provide the user with a collection of data structures and algorithms that simplifies the application development process. Similarly, STAPL provides the Parallel Container Framework (PCF) to facilitate the development of pContainers which are parallel and concurrent data structures.

pContainers are containers that are distributed across a parallel machine and accessed concurrently. A large number of parallel data structures have been proposed in the literature. They are often complex data structures, addressing issues related to data partitioning, distribution, communication, synchronization, load balancing,

The journal model is IEEE Transactions on Automatic Control. 
and thread safety. The complexity of building such structures for every parallel program is one of the main impediments to parallel program development. To alleviate this problem we have developed the STAPL Parallel Container Framework (PCF). It consists of a collection of elementary pContainers and methods to specialize, or compose them into pContainers of arbitrary complexity. Thus, instead of building their distributed containers from scratch in an ad-hoc fashion, programmers can use inheritance to derive new specialized containers and composition to generate complex data structures. Moreover, the PCF provides the mechanisms to enable any container, sequential or parallel, to be used in a distributed fashion without requiring the programmer to deal with concurrency mechanisms such as data distribution or thread safety. Furthermore, when composed, these containers carry over, at every level, their "interesting" features for parallelism.

The STAPL PCF presented in this thesis makes several novel contributions.

- Modular design: Provides a set of classes and rules for using them to build new pContainers and customize existing ones.

- Composition: Supports composition of pContainers that allows the recursive development of complex pContainers that support nested parallelism.

- Interoperability: Provides mechanisms to generate a wrapper for any data structure, sequential or parallel, enabling it to be used in a distributed, concurrent environment.

- Library: It provides a library of basic pContainer constructed using the PCF as initial building blocks.

Some important properties of pContainers supported by the PCF are noted below. 
Shared object view. Each pContainer instance is globally addressable. This supports ease of use, relieving the programmer from managing and dealing with the distribution explicitly, unless desired.

Arbitrary degree and level of parallelism. For pContainers to provide scalable performance on shared and/or distributed memory systems they must support an arbitrary, tunable degree of parallelism, e.g., number of threads. Moreover, given the importance of hierarchical (nested) parallelism for current and foreseeable architectures, it is important for composed pContainers to allow concurrent access to each level of their hierarchy.

Instance-specific customization. The pContainers in the PCF can be dynamic and irregular and can adapt (or be adapted by the user) to their environment. The PCF facilitates the design of pContainers that support advanced customizations so that they can be easily adapted to different parallel applications or even different computation phases of the same application. For example, a pContainer can dynamically change its data distribution or adjust its thread safety policy to optimize the access pattern of the algorithms accessing the elements. Alternatively, the user can request certain policies and implementations which can override the provided defaults or adaptive selections.

Portions of this dissertation have been published in the following papers [63, 65, $15,64,13,68]$.

\section{A. Outline}

The dissertation is outlined as follows. In Chapter II we present related work. In Chapter III we provide an overview of STAPL discussing the main modules of the library and emphasizing the ones that the pContainers will interact directly with: 
parallel view (pView) and run time system (RTS). Chapter IV introduces the notion of a parallel container as a parallel and distributed data structure describing the required functionality that it needs to provide to improve parallel programming productivity. Chapter V provides a detailed description of the Parallel Container Framework and of the individual modules that make up a parallel container. Chapter V, Section E shows an example of STAPL pContainer implemented using the framework describing how all modules introduced in Chapter V interact to provide the overall functionality of a parallel array (pArray) data structure.

Chapter V, Section D introduces the base pContainers provided by the framework. Chapter VI discusses the thread safety support provided by default by all STAPL pContainers and introduces the interfaces that advanced users need to customize in order to implement custom thread safety policies. Chapter VII describes the memory consistency model provided by the pContainers and specifies how additional models can be implemented.

Starting with Chapter VIII we describe individual pContainers implemented using the $\mathrm{PCF}$ and an evaluation of their performance. We describe their derivation relation from the classes of the framework, their interfaces and show experimental results or both pContainer methods and pAlgorithms. Chapter VIII introduces the general methodology used to evaluate the pContainer methods and application performance. Chapter IX discusses the pArray, Chapter X the pList, Chapter XI the pGraph and Chapter XII associative pContainers. Experimental results evaluating the pContainer composition are included in Chapter XIII. We conclude with some final remarks in Chapter XIV. 


\section{CHAPTER II}

\section{RELATED WORK}

There is a large body of work in the area of parallel and distributed data structures with projects aiming at shared memory architectures, distributed memory architectures or both. Parallel programming languages $[19,18,17,70]$ typically provide built in arrays and provide minimal guidance to the user on how to develop their own specific parallel data structures. STAPL pContainers are generic data structures and this characteristic is shared by a number of existing projects such as PSTL [39], TBB [37], and POOMA [53].

There are several parallel libraries that have similar goals to STAPL. Some of the libraries provide application specific data structures that are fine tuned for certain applications. While they achieve high efficiency, lack of generality makes them hard to use in different applications. A large amount of effort has been put into regular data structures, like arrays and matrices, to make them suitable for parallel programming. Irregular data structures, like graph, tree, etc., are not widely studied for this purpose.

PSTL (Parallel Standard Template Library) [39, 40] explores the same underlying philosophy as STAPL, which is to extend the C++ STL for parallel programming. PSTL emphasizes regular data structures, such as vector, multidimensional array, etc., which are more suited for scientific computation. The underlying runtime system provides support for explicit shared memory operations, such as put and get. The data distribution mechanism allows for regular distribution (block distribution) and the containers are treated as place holders for data accessed by stand alone parallel algorithms. PSTL project is unfortunately not maintained anymore. The Amelia Vector Template Library (AVTL) [59], provides a parallel vector data structure, which can be distributed in an uniform fashion. STAPL is different that both PSTL and AVTL, 
providing a larger variety of data structures integrated uniformly in a framework.

Hierarchically Tiled Arrays (HTA) [8, 9] is introduced as a useful programming paradigm where the user writes programs in a single threaded fashion and the data structure takes care of parallelization in a transparent manner. HTA is a parallel data container whose data is partitioned in tiles and can be distributed across different computation servers. Operations on the data are dispatched to the servers owning the data. The most representative characteristic of an HTA is the support for hierarchical partitioning and indexing. Through a flexible indexing scheme for its tiles and elements HTAs allows communication to be expressed as array assignments. Hierarchical data structures can provide more flexibility to the user to express its algorithms, can be used to improve data locality (e.g., tiling[45]), and to express nested parallelism[10]. Some of the HTA concepts have been adopted in STAPL, like providing hierarchical views of the data available in pContainers.

POOMA[53] is a $\mathrm{C}++$ library designed to provide a flexible environment for data parallel programming of scientific applications. POOMA provides a collection of parallel data types together with a set of algorithms geared specifically toward scientific applications. STAPL shares similar goals with POOMA. Code developed using POOMA is intended to be portable, efficient, allows rapid application development by reusing existing components. The data structures provided by POOMA are referred to as "Global Data Types". They are similar to STAPL pContainers but they are oriented toward scientific computing. POOMA provides n-dimensional arrays, vector and matrix classes. STAPL's pContainer infrastructure is more generic providing a larger variety of data structures like graphs, list, hash maps.

Multiphase Specifically Shared Array (MSA) [21] is proposed as a data structure that allows the users to benefit from having a distributed data structure with shared memory interface. MSA is an important data structure developed using Charm++ 
language[41]. To improve the performance of accessing the elements of the MSA the authors optimize for three access patterns that are common in many algorithms: read only, write many and accumulate. The authors emphasize that while a read write mode would be more general it is often hard to guarantee memory consistency without performance loss. The access modes can be interchanged at certain points in the program (synchronization points).

There has been significant research in the field of parallel and concurrent data structures. Much work has focused on providing efficient locking mechanisms and methodologies for transforming existing sequential data structures into concurrent data structures [20, 24, 26, 33, 34]. Valois [69] was one of the first to present a nonblocking singly-linked list data structure by using Compare\&Swap (CAS) synchronization primitives rather than locks. The basic idea is to use auxiliary nodes between each ordinary node to solve the concurrency issues. Subsequent work [31, 48, 27, 51] proposes different concurrent list implementations for shared memory architectures, emphasizing the benefits on non-blocking implementations in comparison with lock based solutions. Investigations of concurrent hash tables [24, 26, 48] and search trees (the most common internal representation for maps and sets) [43, 47] explore efficient storage schemes, different lock implementations, and different locking strategies (e.g., critical sections, non-blocking, wait-free [33]), especially in the context of shared memory architectures. In contrast, the STAPL pContainers are designed to be used in both shared and distributed memory environments and addresses the additional complexity required to manage the data distribution. Ideas pioneered in these papers can be integrated in our framework for efficient concurrent access on a shared memory location.

UPC[25], Titanium[70], Chapel[17] and X10[18] are several other languages that are part of the large family of Partitioned Global Address Space (PGAS) languages. 
They all provide minimal support for parallel data structures in the form of regular arrays. Dynamic data structures like graphs, maps, hash maps are often user responsability with no explicit support from the language. All these languages recognizes the importance of allowing users to customize the data distribution and they provide appropriate support for parallel arrays. In [22] it is mentioned that Chapel intends to provide support for distributions for dynamic data structures, a feature that is supported in our framework.

Table I.: Comparison with related projects.

\begin{tabular}{|c|c|c|c|c|c|}
\hline $\begin{array}{l}\text { Features/ } \\
\text { Project }\end{array}$ & $\begin{array}{l}\text { Paradigm } \\
1\end{array}$ & Architecture & Adaptive & Generic & $\begin{array}{l}\text { Data } \\
\text { Distribution }\end{array}$ \\
\hline STAPL & S/MPMD & Shared/Dist & Yes & Yes & Auto/User \\
\hline PSTL & SPMD & Shared/Dist & No & Yes & Auto \\
\hline Charm++ & MPMD & Shared/Dist & No & Yes & User \\
\hline CILK & S/MPMD & Shared/Dist & No & No & User \\
\hline NESL & S/MPMD & Shared/Dist & No & Yes & User \\
\hline POOMA & SPMD & Shared/Dist & No & Yes & User \\
\hline SPLIT-C & SPMD & Shared/Dist & $\mathrm{Np}$ & No & User \\
\hline $\mathrm{X} 10$ & $\mathrm{~S} / \mathrm{MPMD}$ & Shared/Dist & No & Yes & Auto \\
\hline Chapel & S/MPMD & Shared/Dist & No & Yes & Auto \\
\hline Titanium & S/MPMD & Shared/Dist & No & No & Auto \\
\hline Intel TBB & SPMD & Shared & No & Yes & Auto \\
\hline
\end{tabular}

1 SPMD - Single Program Multiple Data, MPMD - Multiple Program Multiple Data

The STAPL PCF differs from the other languages and libraries by focusing on developing a generic infrastructure that will efficiently provide a shared memory abstraction for pContainers. The framework automates, in a very configurable way, aspects relating to data distribution and thread safety. We emphasize on interoperability with other languages and libraries [15], and we use a compositional approach 
where existing data structures (sequential or concurrent, e.g., TBB containers) can be used as building blocks for implementing parallel containers. We include in Table I a comparison between STAPL and a number of other projects according to a number of criteria. While all the libraries above share a common goal, to make parallel programming easier, STAPL pContainer distinguish itself by placing emphasis on general (regular and irregular) data structures (vector, list, graph, hash, etc), flexible mechanisms to specify data distribution, a shared object view programming paradigm with implicit communication, adaptivity support for both algorithms and containers. 


\section{CHAPTER III}

\section{STAPL OVERVIEW}

STAPL $[16,4,67,5,68,52]$ is a framework for parallel code development in $\mathrm{C}++$. Its core is a library of $\mathrm{C}++$ components with interfaces similar to the (sequential) ISO $\mathrm{C}++$ standard library [49]. STAPL offers to the parallel system programmer a shared object view of the data space. The objects are distributed across the memory hierarchy which can be shared and/or distributed address spaces. Internal STAPL mechanisms assure an automatic translation from one space to another, presenting a unified address space to the less experienced user. For more experienced users, the local/remote distinction of accesses can be exposed and performance enhanced for a specific application or application domain. To exploit large hierarchical systems, such as BlueGene [50], Cray XT5 [56], STAPL allows for (recursive) nested parallelism.

The STAPL infrastructure consists of platform independent and platform dependent components that are revealed to the programmer at an appropriate level of detail through a hierarchy of abstract interfaces (see Figure 1). The platform independent components include the core parallel library, and an abstract interface to the communication library and run-time system. The core STAPL library consists of pAlgorithms (parallel algorithms) and pContainers (distributed data structures) [66]. Important aspects of all STAPL components are extendability and composability. For example, users can extend and specialize STAPL pContainers (using inheritance) and/or compose them. For example, STAPL users can employ pContainers of pContainers in pAlgorithms which may themselves call pAlgorithms.

pContainers, the distributed counterpart of STL containers, are thread-safe, concurrent objects, i.e., shared objects that provide parallel methods that can be invoked concurrently. They are composable and extensible by users via inheritance. Cur- 


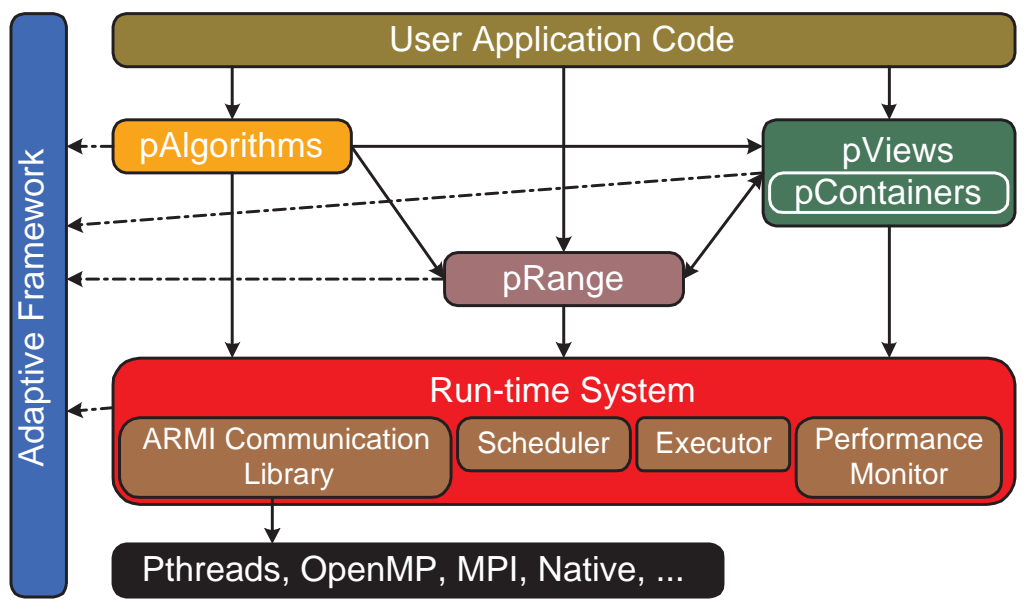

Fig. 1. STAPL overview.

rently, STAPL provides counterparts of all STL containers (e.g., pArray[63], pVector, pList [65], pMap[64], etc.), and pContainers that do not have STL equivalents: parallel matrix (pMatrix [15]) and parallel graph (pGraph). pContainers provide two kinds of methods to access their data: methods which are semantically equivalent to their sequential counterpart and methods which are specific to parallel computations. For example, STAPL provides an insert_async method that can return control to the caller before its execution completes. While a pContainer's data may be distributed, pContainers offer the programmer a shared object view, i.e., they are shared data structures with a global address space. This is provided by an internal object translation mechanism which can transparently locate both local and remote elements. The physical distribution of a pContainer data can be assigned automatically by STAPL or can be user-specified.

A pAlgorithm is the parallel equivalent of an STL algorithm. STAPL currently includes a large collection of parallel algorithms, including parallel counterparts of STL 
algorithms, pAlgorithms for important parallel algorithmic techniques (e.g., prefix sums [38], the Euler tour technique [38]), and some for use with STAPL extensions to STL (i.e., graph algorithms for the pGraph). Analogous to STL algorithms that use iterators, STAPL pAlgorithms are written in terms of pViews $[13,14]$. Briefly, pViews allow the same pContainer to present multiple interfaces to its users, e.g., enabling the same pMatrix to be 'viewed' (or used) as a row-major or column-major matrix or even as linearized vector.

pAlgorithms are represented by pRanges. Briefly, a pRange is a graph whose vertices are tasks and the edges the dependencies, if any, between them. A task includes both work (represented by what we call workfunctions) and data (from pContainers, generically accessed through pViews). The executor, itself a distributed shared object, is responsible for the parallel execution of computations represented by pRanges; as tasks complete, the executor updates dependencies, identifies tasks that are ready for execution, and works with the scheduler to determine which tasks to execute. Nested parallelism can be created by invoking a pAlgorithm from within a task.

The platform dependent STAPL components are mainly contained in the STAPL runtime system (RTS) $[54,55,57,58]$, which provides the API to the OS and several important functions. The RTS includes the communication abstractions that are used by the higher level STAPL components.

In the following two sections we provide more details regarding the STAPL pView concept and the runtime system because they are required to properly describe pContainer functionality. 


\section{A. STAPL pView Concept}

Decoupling of data structures and algorithms is a common practice in generic programming. STL, the $\mathrm{C}++$ Standard Template Library, obtains this abstraction by using iterators, which provide a generic interface for algorithms to access data which is stored in containers. This mechanism enables the same algorithm to operate on multiple containers. In STL, different containers support various types of iterators that provide appropriate functionality for the data structure, and algorithms can specify which types of iterators they can use. The major capability provided by the iterator is a mechanism to traverse the data of a container.

The STAPL pView $[13,14]$ generalizes the iterator concept by providing an abstract data type (ADT) for the data it represents. While an iterator corresponds to a single element, a pView corresponds to a collection of elements. Also, while an iterator primarily provides a traversal mechanism, pViews provide a variety of operations as defined by the ADT. For example, all STAPL pViews support size() operations that provide the number of elements represented by the pView. A STAPL pView can provide operations to return new pViews. For example, a pMatrix supports access to rows, columns, and blocks of its elements through row, column and blocked pViews, respectively.

A primary objective of the pViews is that they are designed to enable parallelism. In particular, each ADT supported by STAPL provides random access to collections of its elements. The size of these collections can be dynamically controlled and typically depends on the desired degree of parallelism. For example, the pList pView provides

concurrent access to segments of the list, where the number of segments could be set to match the number of parallel processes. The pView provides random access to a partitioned data space. This capability is essential for the scalability of STAPL 
programs. To mitigate the potential loss of locality incurred by the flexibility of the random access capability, pViews provide, to the degree possible, a remapping mechanism of a user specified pView to the collection's physical distribution (known as the native pView).

In this section, we first introduce the pView concept and then explain how it can be generalized for the parallel and distributed environment of STAPL. A pView is a class that defines an abstract data type (ADT) for the collection of elements it represents. As an ADT, a pView provides operations to be performed on the collection, such as read, write, insert, and delete.

pViews have reference semantics, meaning that a pView does not own the actual elements of the collection but simply references to them. The collection is typically stored in a pContainer to which the pView refers; this allows a pView to be a relatively light weight object as compared to a container. However, the collection could also be another pView, or an arbitrary object that provides a container interface. With this flexibility, the user can define pViews over pViews, and also pViews that generate values dynamically, read them from a file, etc.

All the operations of a pView must be routed to the underlying collection. To support this, a mapping is needed from elements of the pView to elements of the underlying collection. This is done by assigning a unique identifier to each pView element (assigned by the pView itself); the elements of the collection must also have unique identifiers. Then, the pView specifies a mapping function from the pView's domain (the union of the identifiers of the pView's elements) to the collection's domain (the union of the identifiers of the collection's elements).

More formally, a pView $\mathcal{V}$ is a tuple

$$
\mathcal{V} \stackrel{\text { def }}{=}(\mathcal{C}, \mathcal{D}, \mathcal{F}, \mathcal{O})
$$


where $\mathcal{C}$ represents the underlying collection, $\mathcal{D}$ defines the domain of $\mathcal{V}, \mathcal{F}$ represents the mapping function from $\mathcal{V}$ 's domain to the collection's domain, and $\mathcal{C}$ is the set of operations provided by $\mathcal{V}$.

To support parallel use, the $\mathcal{C}$ and $\mathcal{D}$ components of the pView can be partitioned so that they can be used in parallel. Also, most generally, the mapping function $\mathcal{F}$ and the operations $\mathcal{O}$ can be different for each component of the partition. That is, $\mathcal{C}=\left\{c_{0}, c_{1}, \ldots, c_{n-1}\right\}, \mathcal{D}=\left\{d_{0}, d_{1}, \ldots, d_{n-1}\right\}, \mathcal{F}=\left\{f_{0}, f_{1}, \ldots, f_{n-1}\right\}$, and $\mathcal{O}=\left\{o_{0}, o_{1}, \ldots, o_{n-1}\right\}$. This is a very general definition and not all components are necessarily unique. For example, the mapping functions $f_{i}$ and the operations $o_{i}$ may often be the same for all $0 \leq i<n$. The tuples $\left(c_{i}, d_{i}, f_{i}, o_{i}\right)$ are called the base views (bViews) of the pView $\mathcal{V}$. The pView supports parallelism by enabling random access to its bViews, which can then be used in parallel by pAlgorithms.

Note that we can generate a variety of pViews by selecting appropriate components of the tuple. For instance, it becomes straightforward to define a pView over a subset of elements of a collection, e.g., a pView of a block of a pMatrix or a pView containing only the even elements of an array. As another example, pViews can be implemented that transform one operation into another. This is analogous to backinserter iterators in STL, where a write operation is transformed into a push back invocation in a container.

Example. A common concept in generic programming is a one-dimensional array of size $n$ supporting random access. The pView corresponding to this will have an integer domain $\mathcal{D}=[0, n)$ and operations $\mathcal{O}$ including the random access read and write operators. This pView can be applied to any container by providing a mapping function $\mathcal{F}$ from the domain $\mathcal{D}=[0, n)$ to the desired identifiers of the container. If the container provides the operations, then they can be inherited using the mechanisms 
Table II. STAPL pViews and corresponding operations. tranform_pview implements an overridden read operation that returns the value produced by a user specified function, the other operations depends on the pView the transform pView is applied to. insert_any refers to the special operations provided by STAPL pContainers that insert elements in unspecified positions.

\begin{tabular}{|c|c|c|c|c|c|c|c|}
\hline & 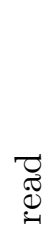 & 志 & $\Xi$ & 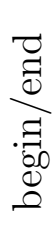 & 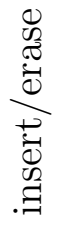 & 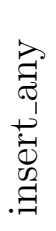 & $\underset{G}{G}$ \\
\hline array_1d_pview & $\mathrm{X}$ & $\mathrm{X}$ & $\mathrm{X}$ & $\mathrm{X}$ & & & \\
\hline array_1d_ro_pview & $\mathrm{X}$ & & $\mathrm{X}$ & $\mathrm{X}$ & & & \\
\hline static_list_pview & $\mathrm{X}$ & & & $\mathrm{X}$ & & & \\
\hline list_pview & $\mathrm{X}$ & $\mathrm{X}$ & & $\mathrm{X}$ & $\mathrm{X}$ & $\mathrm{X}$ & \\
\hline matrix_pview & $\mathrm{X}$ & $\mathrm{X}$ & $\mathrm{X}$ & & & & \\
\hline graph_pview & $\mathrm{X}$ & $\mathrm{X}$ & & & $\mathrm{X}$ & $\mathrm{X}$ & $\mathrm{X}$ \\
\hline strided_1D_pview & $\mathrm{X}$ & $\mathrm{X}$ & $\mathrm{X}$ & $\mathrm{X}$ & & & \\
\hline transform_pview & $\mathrm{O}$ & & - & - & & & \\
\hline balanced_pview & $\mathrm{X}$ & & $\mathrm{X}$ & $\mathrm{X}$ & & & \\
\hline overlap_pview & $\mathrm{X}$ & & $\mathrm{X}$ & $\mathrm{X}$ & & & \\
\hline native_pview & $\mathrm{X}$ & & $\mathrm{X}$ & $\mathrm{X}$ & & & \\
\hline
\end{tabular}

provided in the base pView in STAPL. If new behavior is needed, then the developer can implement it explicitly.

Table II shows an initial list of pViews available in STAPL. Some special cases of pViews are particularly useful in the context of parallel programming. For instance the single-element partition, where the domain of the collection is split into single elements and all mapping functions are identity functions. This is the default partition adopted by STAPL when calling a pAlgorithm to express maximum parallelism.

Other pViews that can be defined include the balanced pView where the data is split into a given number of chunks, and the native pView, where the partitioner 
Overlap pView of $A[0,10]$

For $c=2, l=2$, and $r=1$,

$i$ th element is $A[c \cdot i, c \cdot i+4]$

elements of the overlap pView:

$A[0,4], A[2,6], A[4,8], A[6,10]$

Fig. 2. Overlap pView example. The input is the pContainer $A[0,10]$.

takes information directly from the underlying container and provides bViews that are aligned with the pContainer distribution. This turns out to be very useful in the context of STAPL. Another pView heavily used in STAPL is the overlap pView, in which one element of the pView overlaps another element. This pView is naturally suited for specifying many algorithms, such as adjacent differences, string matching, etc. As an example, we can define an overlap pView for a one-dimensional array $A[0, n-1]$ using three parameters, $c$ (core size), $l$ (left overlap), and $r$ (right overlap), so that the $i$ th element of the overlap pView $v^{o}[i]$ is $A[c \cdot i, c \cdot i+l+c+r-1]$. See example in Figure 2.

The native pView is a pView whose partitioned domain $\mathcal{D}$ matches the data partition of the underlying collection, allowing references to its data to be local. The balanced pView partitions the data set into a user specified number of pieces. This pView can be used to balance the amount of work in a parallel computation. If STAPL algorithms can use balanced or native pViews, then performance is greatly enhanced.

All STAPL pContainers provide native pViews that have the same interface as 
the pContainer. For example, pArray provides array_1d_pview, pList provides p_list_pview, pVector provides p_vector_pview, pGraph provides p_graph_pview, simple associative pContainers provide p_set_pview, pair associative pContainers provide $\mathrm{p}$ _map_pview and pMatrix provides array_2d_pview. Additional pViews with certain ADT can be defined on top of existing data structures. For example a pGraph pView can be defined on top of a pArray of list of edges as shown in [14].

\section{B. Runtime System}

The STAPL runtime system (RTS) $[54,55,57,58]$ is the only platform specific component of the library that needs to be ported to each target architecture. It provides a communication and synchronization library (ARMI), an executor, and a scheduler of the tasks of the pRanges. The RTS is not intended to be used directly by the STAPL user or library developer.

The RTS provides locations as an abstraction of processing elements in a system. A location is a component of a parallel machine that has a contiguous address space and has associated execution capabilities (e.g., threads). Different locations can communicate exclusively through ARMI, the Adaptive Remote Method Invocation library, which represents the communication layer of the RTS. Special types of objects, called p_objects, implement the basic concept of a shared object. The representative of a p_object in each location has to register with the RTS to enable Remote Method Invocations (RMIs) between the representative objects. This is the reason why all the parallel objects in STAPL inherit from the base p_object class. RMIs enable the exchange of data between locations and the transfer of the computation from one location to another.

RMIs are divided into two classes: asynchronous RMIs and synchronous RMIs. 
The former execute a method on a registered object in a remote location without waiting for its termination, while the latter block waiting for the termination of the invoked method. A mechanism is provided to asynchronously execute methods that return values to the caller. As parallel machine sizes reach processor counts into the millions, it becomes essential for algorithms to be implemented using only asynchronous RMIs. In STAPL, these operations implement computation migration, which allows scalability for very large numbers of processors. We also provide sync_rmis for completeness, but their use is discouraged. The RTS guarantees that requests from a location to another location are executed in order of invocation at the source location.

The RTS provides RMI versions of common aggregate operations. These primitives come in two flavors: one-sided, in which a single requesting location invokes the execution of a method in all others, eventually receiving a result back, and collective, in which all locations participate in the execution of the operation. All the RMI operations, point-to-point, single-sided, and collective, are defined within communication groups, thus enabling nested parallelism. Collective operations have the same semantics as the traditional MPI collective operations. The provided operations include broadcast, reduce, and fence. The fence operation, called rmi_fence, when completed, guarantees that no pending RMIs are still executing in the group where it is called. This is essential for guaranteeing correctness of phases of computations that have to be completed before the next one can start.

The RTS provides some optimizations to use bandwidth and reduce overhead. The major techniques used are aggregation, that packs multiple requests to a given location into a single message, and combining, that supports the repetitive execution of the same method in a given location without incurring a large overhead for object construction and function calls. Memory management and the number of messages 
aggregated are managed by the RTS adaptively according to the application needs. Another RTS component, the executor, has the role of executing task graphs corresponding to pAlgorithms. The executor identifies sets of independent tasks to be executed, and schedules them according to the customizable scheduler module. From its perspective, the executor treats incoming RMI requests and algorithmic tasks as RTS tasks. Tasks can be assigned to execution threads and they are considered independent. 


\section{CHAPTER IV}

\section{PARALLEL CONTAINER}

Data structures are essential building blocks of any generic programming library. Sequential libraries like STL [49], Leda[44], BGL [60], and MTL [61, 28], provide to the user a collection of data structures and algorithms. For simple regular data structures such as arrays, vectors, and lists, the implementation may be relatively straightforward. More complicated data structures such as matrices (MTL) or graphs (BGL) require the developer to consider a modular design with different functional units that allows customization of different aspects of the data structure to improve the performance of the algorithms. For example, the users of a matrix may want dense or sparse storage and the layout in memory to be row or column oriented.

In a multiprocessor environment, the complexity of a data structure increases due to a number of challenges which are not present in sequential computing. For example, there are issues related to data management such as partitioning, distribution, communication, synchronization, load balancing, and thread safety that have to be considered. To minimize the user's effort in dealing with all these factors, we have developed the STAPL Parallel Container Framework (PCF) which consists of a set of formally defined concepts and a methodology for developing generic parallel containers starting from sequential, STL-like containers. Users, by implementing the appropriate interfaces, can assemble with minimal effort a data structure that will provide methods to build and access a distributed collection of elements.

\section{A. pContainer Requirements}

Design requirements of the STAPL pContainers developed within the PCF include: scalable performance, a clearly specified memory consistency model, a shared object 
view, thread safety, composition, and adaptivity.

- Scalable performance. pContainers must provide scalable performance on shared and/or distributed memory systems. The performance of the pContainer methods must achieve the best known parallel complexity. This is obtained by efficient algorithms coupled with non-replicated, distributed data structures that allow a degree of concurrent access proportional to the degree of desired parallelism, e.g., the number of threads.

- Thread safety and memory consistency model. When needed, the pContainer must be able to provide thread safe behavior and it must respect a well specified memory consistency model as discussed in Chapter VI and Chapter VII, respectively. When a level of a pContainer is distributed across shared memory where multiple threads can access and modify it, the PCF must ensure thread safety.

- Shared object view. Each pContainer instance is globally addressable, i.e., it provides a shared memory address space (Chapter V, Section C). Individual pContainer elements can be accessed from any computation thread independent of their physical location. This supports ease of programming, allowing programmers to ignore the distributed aspects of the container if they so desire.

- Composition. The capability to compose pContainers (i.e., build pContainers of pContainers) provides a natural way to express and exploit nested parallelism while preserving locality. New pContainers can be created by composing existing pContainers, e.g., a pVector of pLists, which would be one way to implement an adjacency list representation of a graph. This feature is not supported by other general purpose parallel libraries. pContainer composition is 
discussed in Section C.

- Adaptivity. A design requirement of the STAPL pContainer is that it can easily be adapted to the data, the computation and the system. For example, different storage options can be used for dense or sparse matrices or graphs or the data distribution may be modified during program execution if access patterns change.

\section{B. pContainer Definition}

A STAPL $p$ Container is a distributed data structure that holds a finite collection of elements $\mathcal{C}$, each with a unique global identifier (GID), their associated storage $\mathcal{S}$, and an interface $\mathcal{O}$ (methods or operations) that can be applied to the collection. The interface $\mathcal{O}$ specifies an Abstract Data Type (ADT), and typically includes methods to read, write, insert or delete elements and methods that are specific to the individual container (e.g., splice for a pList or out_degree for a pGraph vertex).

The pContainer also includes meta information supporting data distribution: a domain $\mathcal{D}$, that is the union of the GIDs of the container's elements, and a mapping $\mathcal{F}$ from the container's domain to the storage. To support parallel use in a distributed setting, the collection $\mathcal{C}$ and the domain $\mathcal{D}$ are partitioned in a manner that is aligned with the storage of the container's elements. The sets $\mathcal{C}, \mathcal{D}$ and $\mathcal{S}$ are isomorphic. For each element of the collection $\mathcal{C}$ there is a unique GID in the domain $\mathcal{D}$ and a unique memory storage in $\mathcal{S}$.

Definition 1. A pContainer is defined as:

$$
p C \stackrel{\text { def }}{=}(\mathcal{C}, \mathcal{D}, \mathcal{F}, \mathcal{O}, \mathcal{S})
$$

The tuple $(\mathcal{C}, \mathcal{D}, \mathcal{F}, \mathcal{O})$ is known as the native pView of the pContainer. As in- 
troduced in Chapter III, Section A and described in more detail in [14], STAPL pViews generalize the iterator concept and enable parallelism by providing random access to collections of their elements. In pViews, the partition of $\mathcal{D}$ can be dynamically controlled and depends on the needs of the algorithm (e.g., a column-based partition of a pMatrix for an algorithm that processes the matrix by columns) and the desired degree of parallelism (e.g., one partition for each core). The native pView associated with a pContainer is a special view in which the partitioned domain $\mathcal{D}$ is aligned with the distribution of the container's data. Performance is enhanced for algorithms that can use native pViews.

We formally introduce now the concepts that were briefly introduced in this section: Global Identifier (GID), Domain, and Partition. Since data structures are collections of elements we start first by introducing some elementary theory about sets and then properly introduce the PCF concepts used to implement a distributed collection of elements.

\section{Set Theory Definitions}

We often refer in this document to the notion of a collection of elements or identifiers and discuss different properties associated with them. In this section we introduce the notions of set and ordered set to facilitate subsequent discussions about containers.

Definition 2. A set is a collection $S$, of distinct objects $\left\{e_{0}, e_{1}, \ldots, e_{i} \ldots\right\}$, which are called the elements of $S$. If $e$ is an element (or member) of $S$, we write $e \in S$.

For example, we can define sets of integer numbers $S_{I}=\{2,7,5\}$, strings $S_{s}=$ $\left\{{ }^{\prime}\right.$ Red $\left.^{\prime},{ }^{\prime} B l u e^{\prime},{ }^{\prime} B l a c k^{\prime}\right\}$ or memory addresses $S_{A}=\{0 x a 0,0 x a 4,0 x a 8\}$.

Definition 3. For a given type $T$, the universe of type $T$ (Universe $(T)$ ) denotes the maximal set of distinct elements that are of type $T$. 
Definition 4. The cardinality $|S|$ of a set $S$ is the number of elements of $S$. If the cardinality of a set is finite, then we call it a finite set. If the cardinality is infinite, then we call it an infinite set. We use \{\} or $\phi$ to denote an empty set, and its cardinality is zero.

We introduce the notion of relations between elements of a set to express ordering and traversals of the elements of a set:

Definition 5. A set of elements $S$, is called a partial ordered set if there is a binary relation $R$ defined on it that is reflexive $(\forall a \in R, a R a$ ), antisymmetric (if $a R b$ and $b R a$ then $a=b$ ) and transitive (if $a R b$ and $b R c$ then $a R c$ ). If the relation $R$ is antisymmetric, transitive and total $(\forall a, b \in S, a R b$ or $b R a)$, than it is a total ordered set.

For example, for $S_{i}=\{2,7,5\}$, we can define a total ordered set by associating correspondingly the $\leq$ relation on integer numbers. For $S_{s}=\{$ Red", "Blue", "Black" $\}$ we can define a total order relation using lexicographical compare for strings.

For a finite total ordered set we can define the following useful notions:

Definition 6. The first, last, next, prev element and unique enumeration (linearization) imposed by a total order relation, $R$, on a finite set $S$, are defined as follows:

1. The first element of the set, first $: S \rightarrow S$, first $(S)=x$, such that $\forall z \in$ $S, x R z$

2. The last element of the set, last $: S \rightarrow S, \operatorname{last}(S)=y$, such that $\forall z \in S, z R y$

3. The next element of an element, next $: S \rightarrow S$, next $(x)=y$, such that $x$ Ry and there is no other $z \in S, x R z$ and $x R y$

4. The previous element of an element, prev $: S \rightarrow S$, prev $(x)=y$, such that $x$ Ry and there is no other $z \in S, y R z$ and $z R x$ 
5. A unique enumeration (linearization) imposed by $R$. Starting from the first element $e_{0}$ there is a unique enumeration $e_{0} R e_{1} R \ldots R e_{n-1}$ that contains all elements in S.

Linearizations are used to specify various traversals of the elements in a set. As described in the following sections linearizations will be used by a pContainer to specify various traversals of its elements.

\section{2. pContainer Domain}

For each pContainer element there is a unique identifier or GID associated with it.

Definition 7. A domain is a set of GIDs.

Throughout $\mathrm{PCF}$ we use various types of domains with different properties that we properly introduce here. For a static container, the number of elements is fixed at the construction time. For dynamic containers, the number of elements, in general, is bounded only by the amount of available storage, and so in principle can be infinite. We distinguish then, for dynamic pContainers, the pContainer's domain which is the universe of all GIDs that will identify its elements and the pContainer's domain instance which is the set of GIDs that identifies the current elements of the pContainer. Hence, the dynamic pContainer domain is infinite while the domain instance is always a finite domain.

\section{Ordered Domain}

The domain as defined in Section 2 does not specify any requirement on the order of the GIDs it contains. To specify an order we introduce the notion of ordered domain.

Definition 8. A domain with a binary relation $R$ on its elements that is reflexive, antisymmetric and transitive is called a partial ordered domain. If the relation $R$ is 
antisymmetric, transitive and total than we have a total ordered domain. We will use the notation $O D \stackrel{\text { def }}{=}(D, R)$ to represent an ordered domain.

In this document, whenever we refer to ordered domains they are total ordered domains, unless otherwise specified. An often used relation is the total order $\leq$ on integers. For example for a domain $D=\{2,1,3\}$ we can have $(D, \leq)=\{1 \leq 2 \leq 3\}$ as an ordered domain. An ordered pContainer domain is often used in the PCF to specify how the pContainer's data is organized in memory and to specify a linear traversal order for the elements.

\section{Example of Domains used by pContainers}

The domain is an important concept that users and developers can interact with while using the PCF. Different pContainers require specific domains and specific implementations to guarantee certain properties, such as the time to perform an operation. In the following, we describe the domains that are provided and used by our framework:

\section{Finite Domains:}

1. An enumeration of individual elements.

Examples: $D=\{1,3,2\}$ or $D=\{a, c, b\}$

Enumerations are considered unordered. A straightforward order can be implied for these domains, by considering the order in which they are specified. Other orders such as $\leq$ or $\geq$ are possible;

2. Range: a sub-domain of a bigger domain. We will represent a range by a first element, a last element and a next operator that allows us to enumerate all its GIDs. 
Examples: $1 D$ Range $=\{[0 . .2), \leq\}=\{0,1\}$ as a sub-domain of the integers domain.

2 DRange $=[(0,0),(2,2))$. A 2DRange can be ordered row-wise or column-wise to obtain a total ordered domain. 2dRange_row $=\{(0,0),(0,1),(1,0),(1,1)\}$ and 2dRange_column $=\{(0,0),(1,0),(0,1),(1,1)\}$

Infinite Domains:

1. Open ordered domains for associative containers, $\left\{\left([\right.\right.$ key 1, key 2$)$, lexicographicalcompare $\left.\left(\leq_{s}\right)\right\}$

Example: Strings domain defined on a set of characters, between "a" and "c", $\{[" a ", " c ")$, lexicographical order $)\}$, contains an infinity of elements (e.g., \{"a","aa", , aaa", "ab","aba", ..\}).

Domains defined as compositions of existing domains:

1. Cartesian products over ordered domains, Ordered $=\left(\left[\left(D_{1}, \leq_{1}\right),\left(D_{2}, \leq_{2}\right)\right], \quad R=\right.$ lexicographical order based on $\left.\leq_{1}, \leq_{2}\right)$

Example: Ordered $D=([(0 . .10, \leq),(0 . .10, \leq)], s R=$ lexicographical order over integer pairs. The gids are pairs $(i, j)$ and $(i, j) \leq(p, q)$ if $i<p$ or if $i==p$ and $j \leq q$

2. Set operations on exiting ordered domains, $O D_{3}=O D_{1}$ op $O D_{2}$ where op $=$ $\{\cap, \cup,-\}$ and the restrictions that $O D_{1}$ and $O D_{2}$ are defined over the same type of GIDs. If $O D_{1}$ and $O D_{2}$ are ordered according to the same relation $R$, then $O D_{3}$ is also ordered according to $\mathrm{R}$.

3. Filtered domain:

$$
D=\left(D_{1}, \text { filter }_{-} \text {function }\right)=\{y \mid y \in D 1 \text { and filter_function }(y)=\text { true }\}
$$


Ex: $D=(([0 . .10], \leq), f=$ every_second_element $)$ defines every second element in the enumeration of the original domain according to $\leq$.

For the ordered domains we mentioned that the last gid is not part of the domain. This last element is a convention (e.g., predefined value type) that has the property that all other elements of the domain are in relation with it. For integral types this value is predefined by our framework but for other gid types the users will have to define the last element as part of the domain interface.

\section{Partition}

The pContainer manages a distributed storage where individual locations store a subset of its elements. The pContainer uses a partition to group its elements in individual units of storage. The partition specifies a decomposition of a domain into sub-domains and how to map an individual GID to the sub-domain that contains it. Later, in Chapter V, Section C.4, we will describe additional functionality that partitions provide.

Definition 9. A partition $P=\left\{D_{0}, D_{1}, . ., D_{n-1}\right\}$ of a domain $D$ is a collection of sub-domains of $D$, such that:

1. $D=D_{0} \cup D_{1} \cup \ldots \cup D_{n-1}$ (the union of the elements of all sub-domains is the set of elements of the original domain)

2. $D i \cap D j=\emptyset, \forall i, j, 0<=i, j<n, i \neq j$ (sub-domains are disjoint)

3. The sub-domain set, $\left\{D_{0}, D_{1}, . ., D_{n-1}\right\}$ is ordered by the relation $\leq$ over domain indices $\left(D_{0} \leq D_{1} \leq, . ., \leq D_{n-1}\right)$.

Partition properties (interface):

1. Partition a domain D into a set of sub-domains according to Definition 9. 
2. Specify the cardinality of the sub-domain set (e.g., how many sub-domains the partition defines).

3. Given an element, identify the sub-domain to which it is associated.

\section{Ordered Partition of Total Ordered Domains}

For a STAPL pContainer that is the parallel equivalent of a STL container we need to support a total order among its elements in order to provide a linearization of its data. For this reason we describe in this section the notion of an ordered partition that is used by the pContainer to provide a linearization of its data.

Definition 10. A partition of a total ordered domain $O D(D, R), P=\left\{\left(D_{0}, R\right), \ldots\right.$, $\left.\left(D_{n-1}, R\right)\right\}$ is an Ordered Partition $(O P)$ if $\forall D_{i} \in P,\left(D_{i}, R\right)$ are total ordered domains and there is a relation $R_{D}$ across the domains of $P$, such that, $\forall x, y \in D$ and $x R y$ then either:

1. $x$ and $y$ belong to the same sub-domain $D_{i}$ and $x R y$ OR

2. $x$ and $y$ belong to different sub-domains $D_{i}$ and $D_{j}$ respectively, and $D_{i} R_{D} D_{j}$

We are using the notation $\left(P, R, R_{D}\right)$ to denote an ordered partition. An ordered partition of a total ordered domain preserves the order among elements from the domain to the sub-domains.

Definition 11. Given a totally ordered domain $O D \equiv(D, R)$ we define the split as a blocked partition $P=\left\{D_{0}, \ldots, D_{n-1}\right\}$ of $\mathrm{D}$ such that $D_{0}$ contains the first $\left|D_{0}\right|$ elements from $\mathrm{D}$ according to $\mathrm{R}, D_{1}$ the next $\left|D_{1}\right|$ elements from $\mathrm{D}$, etc.

The split is a blocking of the unique enumeration of a total ordered domain and it is an important way of defining a partition that preserves the relation between elements in the original domain. For example, for the totally ordered domain 
$([0,10), \leq)$ we can have $P=\left\{D_{0}=([0,5), \leq), D_{1}=([5,10), \leq)\right\}$ and $R_{D} \equiv D_{0} \leq D_{1}$ as a possible split.

A split of a total ordered domain and the relation $R_{D} \equiv D_{0} \leq \ldots \leq D_{n-1}$ induces an ordered partition. The split can be seen as a top-down procedure of specifying an ordered partition for an ordered domain. Next we show how to specify an ordered domain corresponding to a set of ordered domains.

Lemma 1. Given a set of ordered domains $P=\left\{\left(D_{0}, R\right), \ldots,\left(D_{n-1}, R\right)\right\}$, and a total relation across the domains, $R_{D}$, then $P$ and $R_{D}$ defines an ordered partition of the total ordered domain $\mathrm{OD}(\mathrm{D}, \mathrm{R})$, where $D=\bigcup_{i=0 . . n-1} D_{i}$ and $\mathrm{R}$ is defined as:

1. if $x$ and $y$ belong to the same domain $D_{i}$ then $x R y \equiv x R y$

2. if $x$ and $y$ belong to different domains $D_{i}$ and $D_{j}$ then $x R y \equiv D_{i} R_{D} D_{j}$

The proof for Lemma 1 is immediate from the definition of the ordered domains. To exemplify how Lemma 1 is useful let us consider two totally ordered domains $D_{0}=\{" R e d ", "$ Blue" $\}, R_{0}="$ Red" $\leq$ "Blue" and $D_{1}=\{"$ Black", "White" $\}$, $R_{1}="$ Black" $\leq$ "White" and $R_{D}=D_{1} \leq D_{0}$. Then $\left\{\left(D_{0}, R_{0}\right),\left(D_{1}, R_{1}\right)\right\}, R_{D}$ is an ordered partition of the totally ordered domain $D=\{"$ Red", "Blue","Black","White" $\}, R=$ "Red" $\leq$ "Blue" $\leq$ "Black" $\leq$ "White" 


\section{C. pContainer Composability}

There are many common data structures that are naturally described as compositions of existing structures. For example, a pVector of pLists provides a natural adjacency list representation of a graph. To enable the construction and use of such data structures, we require that the composition of pContainers be a pContainer, i.e., that pContainers are closed under composition.

An important feature of composed pContainers is that they support hierarchical parallelism in a natural way - each level of the nested parallel constructs can work on a corresponding level of the pContainer hierarchy. If well matched by the machine hierarchy, this can preserve existing locality and improve scalability.

In this section, we examine the properties of the composed pContainer, and analyze the relations between the pContainers that are composed and the final composed pContainer. As a simple example, consider a pArray of pArrays. The following code is used to declare the composed pArray and correspondingly resize each of the nested pArrays to obtain the hierarchy depicted in Figure 3 :

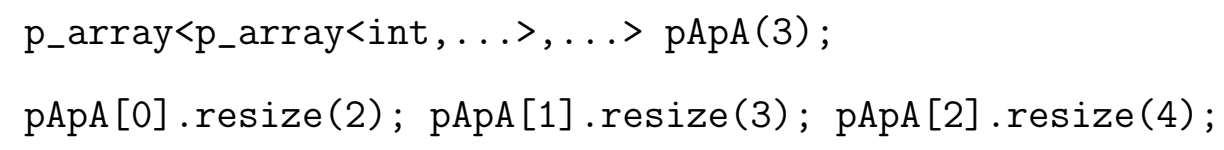

The composed data structure obtained using composition can be thought of as a single data structure whose domain, interface, storage, etc., are compositions of the corresponding modules at the two levels of the hierarchy. For example, the domains of the nested pArrays depicted in Figure 3 are $\mathcal{D}_{10}=\{0,1\}, \mathcal{D}_{11}=\{0,1,2\}$ and $\mathcal{D}_{12}=$ $\{0,1,2,3\}$ and the domain of the outer pArray is $\mathcal{D}_{0}=\{0,1,2\}$. The composed data structure however can be viewed as a data structure by itself where the domain consists of the following GIDs: $\mathcal{D}_{\text {composed }}=\{(0,0),(0,1),(1,0),(1,1),(1,2),(2,0),(2,1)$, $(2,2),(2,3)\}$. We formally describe the domain of the composed pContainer as the 


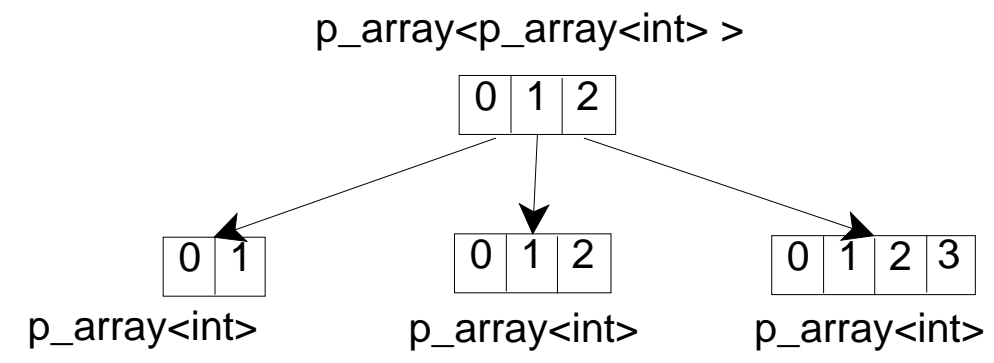

Fig. 3. Composed pArray of pArrays.

union of the cross products of each element in $\mathcal{D}$ with the corresponding domains of the nested pContainers.

$$
\begin{aligned}
\mathcal{D}_{\text {composed }} & =\bigcup_{i \in \mathcal{D}}\left(\left\{\mathcal{D}_{0}[i]\right\} \times \mathcal{D}_{1 i}\right) \\
& =\left(\{0\} \times \mathcal{D}_{10}\right) \cup\left(\{1\} \times \mathcal{D}_{11}\right) \cup\left(\{2\} \times \mathcal{D}_{12}\right) \\
& =\{(0,0),(0,1),(1,0),(1,1),(1,2),(2,0),(2,1),(2,2),(2,3)\}
\end{aligned}
$$

The corresponding interface of the composed container can be thought of as the composition of the interfaces of the pArrays that are composed. For example accessing the element corresponding to GID $(1,0)$ in the composed container can be done with the following invocation: pApA.get_element(1).get_element(0). The method of the composed pArray is naturally an application in series of methods at both levels of the hierarchy.

We define the height of a composed pContainer as the number of pContainers present in the composed type. For a pContainer whose element is a non pContainer type, the height is defined as one. For the composed pArray example in Figure 3, the height of the composition is two. The composition can be done an arbitrary 

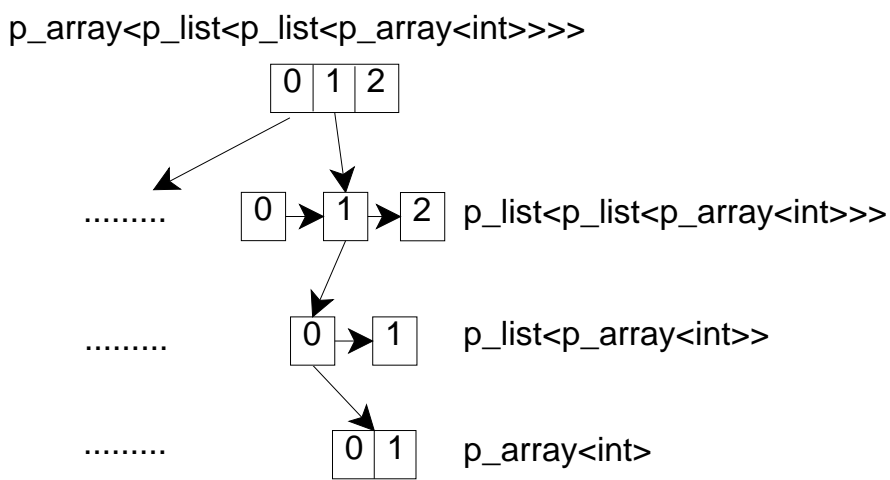

Fig. 4. Composed pContainers.

number of times and include various types of pContainers. For example, composing a pArray $<$ pList $<\mathrm{T}>>$ with a pList $<$ pArray $<\mathrm{T}>>$ results in the composed pContainer pArray $<$ pList $<$ pList $<$ pArray $<$ T $>>>>$ with height four. In Figure 4, we include the hierarchical organization of a possible instance of such a composed pContainer.

In the remainder of this section we formalize the process of composing two arbitrary pContainers that themselves can be the result of a previous composition.

Definition 12. Let $p C_{1}=\left(\mathcal{C}_{1}, \mathcal{D}_{1}, \mathcal{F}_{1}, \mathcal{O}_{1}, \mathcal{S}_{1}\right)$ and $p C_{2}=\left(\mathcal{C}_{2}, \mathcal{D}_{2}, \mathcal{F}_{2}, \mathcal{O}_{2}, \mathcal{S}_{2}\right)$ be two composed pContainers of height $H_{1}$ and $H_{2}$, respectively. The composed pContainer $p C=p C_{1} \circ p C_{2}$ is of height $H=H_{1}+H_{2}$. In $p C$, each element of $p C_{1}, p C_{1}[i], i \in \mathcal{D}_{1}$, is an instance of $p C_{2}$, called $p C_{2 i}=\left(\mathcal{C}_{2 i}, \mathcal{D}_{2 i}, \mathcal{F}_{2 i}, \mathcal{O}_{2 i}, \mathcal{S}_{2 i}\right)$.

Each component of $p C$ is derived appropriately from the corresponding components of $p C_{1}$ and $p C_{2}$. For example, in the special case when all the mapping functions 
$\mathcal{F}_{2 i}$ and operations $\mathcal{O}_{2 i}$ are the same, we have

$$
\begin{aligned}
\mathcal{D} & =\bigcup_{i \in \mathcal{D}_{1}}\left(\left\{\mathcal{D}_{1}[i]\right\} \times \mathcal{D}_{2 i}\right) \\
\mathcal{F} & =\left(\mathcal{F}_{1}, \mathcal{F}_{2}\right) \\
\mathcal{O} & =\left(\mathcal{O}_{1}, \mathcal{O}_{2}\right)
\end{aligned}
$$

where, for $(x, y) \in \mathcal{D}, \mathcal{F}(x, y)=\left(\mathcal{F}_{1}, \mathcal{F}_{2}\right)(x, y)=\left(\mathcal{F}_{1}(x), \mathcal{F}_{2}(y)\right)$. The components $\mathcal{C}$ and $\mathcal{S}$ are isomorphic to $\mathcal{D}$ and they are defined similarly (for each element of the collection, there is a unique GID and a unique storage). With this formalism, arbitrarily deep hierarchies can be defined by recursively composing pContainers.

Given a composed pContainer $P C=(\mathcal{C}, \mathcal{D}, \mathcal{F}, \mathcal{O}, \mathcal{S})$, with a hierarchy of height $H$, we need a mapping function to access a pContainer at level $1 \leq h \leq H$. This function is a sub-sequence (prefix) of the tuple of functions $\mathcal{F}, \mathcal{F}^{h}\left(x_{1}, \ldots, x_{h}\right)=$ $\left(\mathcal{F}_{1}\left(x_{1}\right), \mathcal{F}_{2}\left(x_{2}\right), \ldots, \mathcal{F}_{h}\left(x_{h}\right)\right)$. The operations available at level $h$ are $\mathcal{O}_{h}$. The pContainer composition is made without loss of information, preserving the meta information of its components in the same hierarchical manner. For example, if two distributed pContainers are composed, then the distribution information of the initial pContainers will be naturally preserved in the new pContainer. In Figure 4 we show an example of a hierarchy with four levels, where levels are counted from top down. Accessing an element at level 3 of the hierarchy is possible with the following interface:

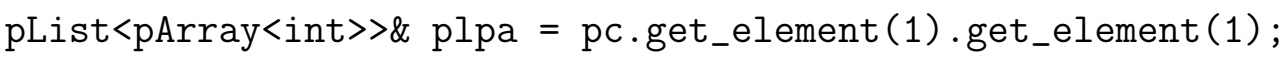

The pList<pArray<int >> reference obtained with the composed method invocation above is possible using the mapping functions of the pContainers at level 1 and 2 of the hierarchy.

A possible research direction for pContainer composition is to allow for (static) 
specialization of the mapping functions if machine information is provided. For example, if the lower (bottom) level of the composed pContainer is distributed across a single shared memory node, then its mapping $\mathcal{F}$ can be specialized for this environment, e.g., some methods may turn into empty function calls. 


\section{CHAPTER V}

\section{PARALLEL CONTAINER FRAMEWORK}

One of the main objectives of the STAPL Parallel Container Framework (PCF) is to simplify the process of developing generic parallel containers as defined in Chapter IV. The PCF is a collection of classes that can be used to construct new pContainers through inheritance and specializations that are customized for the programmer's needs while preserving the properties of the base container. In particular, the PCF can generate a wrapper for any standard data structure, sequential or parallel, that has the meta information necessary to use the data structure in a distributed, concurrent environment. This allows the programmer to concentrate on the semantics of the container instead of its concurrency and distribution management. Thus, the PCF makes developing a pContainer almost as easy as developing its sequential counterpart. Moreover, the PCF facilitates interoperability by enabling the use of parallel or sequential containers from other libraries, e.g., MTL [28], BGL [30] or TBB [36].

STAPL provides a library of pContainers constructed using the PCF. These include counterparts of STL containers (e.g., pVector, pList [65], and associative containers [64] such as pSet, pMap, pHashMap, pMultiSet, pMultiMap) and additional containers such as pArray [63], pMatrix [15], and pGraph.

Novice programmers can immediately use the available data structures with their default settings. More sophisticated parallel programmers can customize or extend the default behavior to further improve the performance of their applications. If desired, this customization can be modified by the programmer for every pContainer instance.

The STAPL Parallel Container Framework has been designed in a modular fashion. This allows developers to implement new pContainers or customize existing 


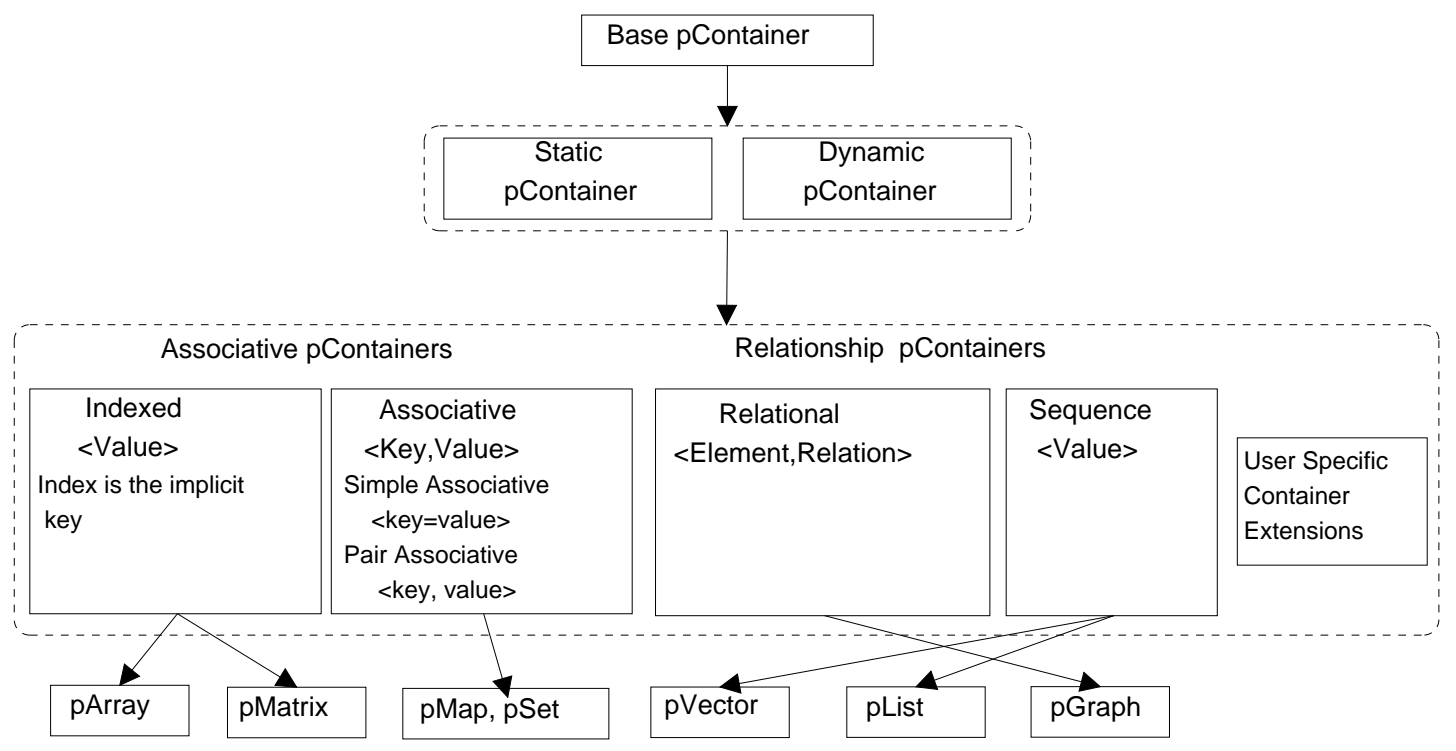

Fig. 5. PCF design.

ones by implementing the appropriate set of interfaces.

\section{A. pContainer Framework Design}

The PCF is designed to allow users to easily build pContainers by inheriting from appropriate modules. It includes a set of base classes representing common data structure features and rules for how to use them to build pContainers. Figure 5 shows the main concepts and the derivation relations between them; also shown are the STAPL pContainers that are defined using those concepts.

All STAPL pContainers derive from p_container_base class. This class is in charge of storing the data using a location-manager and data distribution information using a data-distribution-manager. It provides a simple interface to initialize the pContainer based on the traits class provided as a template argument, and a domain and partition instance. The complete interface is described in Section D.1. 
The remaining classes in the PCF provide additional interfaces and requirements. First, static and dynamic pContainers are classes to indicate if elements can be added to or removed from the pContainer. The property that the number of elements is fixed allows for more efficient implementations of domains, partitions and pViews to be used. The interfaces are discussed in Sections D.2 and D.3.

The next discrimination is between associative (Section D.5) and relational (Section D.6) pContainers. In associative containers, there is an implicit or explicit association between a key and a value. For example, in an array there is an implicit association between the index and the element corresponding to that index; we refer to such (multi-dimensional) arrays as indexed pContainers (Section D.4). In other cases, such as a hashmap, keys must be stored explicitly. The PCF provides an associative base pContainer for such cases. The relational pContainers include data structures that can be expressed as a collection of elements and relations between them. This includes graphs and trees, where the relations are explicit and may have values associated with them (e.g., weights on the edges of a graph), and lists where the relations between elements are implicit.

All classes of the PCF have well defined interfaces as described in Section D and default implementations that can be customized for each pContainer instance using template arguments called traits. This allows users to specialize various aspects, e.g., the data distribution, to improve the performance of their data structures. The pContainer customization using traits is further discussed in Section H.

\section{B. pContainer Interfaces}

STAPL pContainers are commonly extensions of existing sequential data structures and they often support the same interface as the sequential counterpart. However it 
is often the case that a pContainer provides an extended interface to better optimize for parallelism. Relative to a sequential data structure the methods provided by a pContainer have more complex semantics that need to be understood correctly to reason about the correctness of an application that is using them. We distinguish the following categories of methods:

Collective methods: These methods have to be invoked on all locations where there is a pContainer representative in an Single Program Multiple Data (SPMD) fashion. Examples of such methods are constructors, destructors and methods that redistribute the pContainer's data. A location cannot invoke another method until the pending method completes.

Element-wise methods: The methods in this category operate on an individual pContainer element and include methods such as get_element, set_element, split_phase_get_element, insert, erase, etc. The element-wise methods are split in the following three categories based on the guarantees we provide about their completions:

- Synchronous methods: have a return type and guarantee that the method is executed and the result available when they return. A thread cannot invoke another method until the pending method completes.

- Asynchronous methods: have no return value and return immediately to the calling thread. The execution will complete subsequently.

- Split phase methods: execution is similar to Charm++ [41], X10 [18]. The return type of a split phase method is a future that allocates space for the result. The invocation returns immediately to the user. When the get method is invoked on the future, the calling thread will return immediately if the result is available or block until the result arrives. This type of method may benefit an 
application if additional work can be performed while waiting for the result and is provided as an alternative to synchronous methods. If one of the phases of a split method has been invoked, then the thread cannot invoke another method until that phase completes.

Global property methods: These methods include operations returning global properties about the data structure such as size() or empty(). These are not collective, synchronous operations but they do require partial information from all locations where there is a pContainer representative. A thread cannot invoke another method until the pending method completes.

New methods facilitating parallel use: For certain pContainers we extend their interfaces with methods that improve their efficiency in a parallel environment. For example, the insert_anywhere method of a pList adds an element to the pContainer to an unspecified position. The pContainer, in such situations may optimize the insertion to improve load balance and speed.

The STAPL runtime provides a fence construct that when invoked guarantees that all pending pContainer methods are completed. Each category of methods described above provides different guarantees about the completion of the invocations and ordering among them when invoked concurrently from multiple threads. The complete specification of the guarantees provided to the user makes the pContainer memory consistency model (MCM) discussed in detail in Chapter VII.

The information about the particular semantics of an element-wise method is embedded in the return type. For example, the parallel array data structure available in STAPL (described in more detail in Chapter IX) provides a simple interface to access elements based on their indices. The interface contains:

void set_element(gid, value); 
value get_element(gid);

pc_future<value> split_phase_get_element(gid);

Based on the signature a user will be aware that set_element is implemented asynchronously (b/c no return type), get_element is synchronous (returns a value) and split_phase_get_element is implemented using a split phase execution. More details on the semantics of the three methods and guarantees about their completion are included in Chapter VII where we discuss the memory consistency model for pContainers. The performance trade offs between these three categories of methods are discussed for various pContainers in Chapters IX, X, XI and XII.

\section{Shared Object View Implementation}

As depicted in Figure 6, a pContainer stores its elements in a non-replicated fashion in a distributed collection of base containers (bContainers, Section C.1). pContainers can be constructed from any existing container, sequential or parallel, so long as it can support the required interface as specified in Section C.1. The pContainers currently provided in STAPL use the corresponding STL containers (e.g., the STAPL pVector uses the STL vector), containers from other sequential libraries (e.g., MTL for matrices), containers available in libraries developed for multicore (e.g., TBB concurrent containers), or other pContainers. This flexibility allows for code reuse and supports interoperability with other libraries.

The pContainer provides a shared object view that enables programmers to ignore the distributed aspects of the container if they so desire. When a hardware mechanism is not available, the shared object view is provided by a software address resolution mechanism that first identifies the bContainer containing the required element and then invokes the bContainer methods in an appropriate manner to 


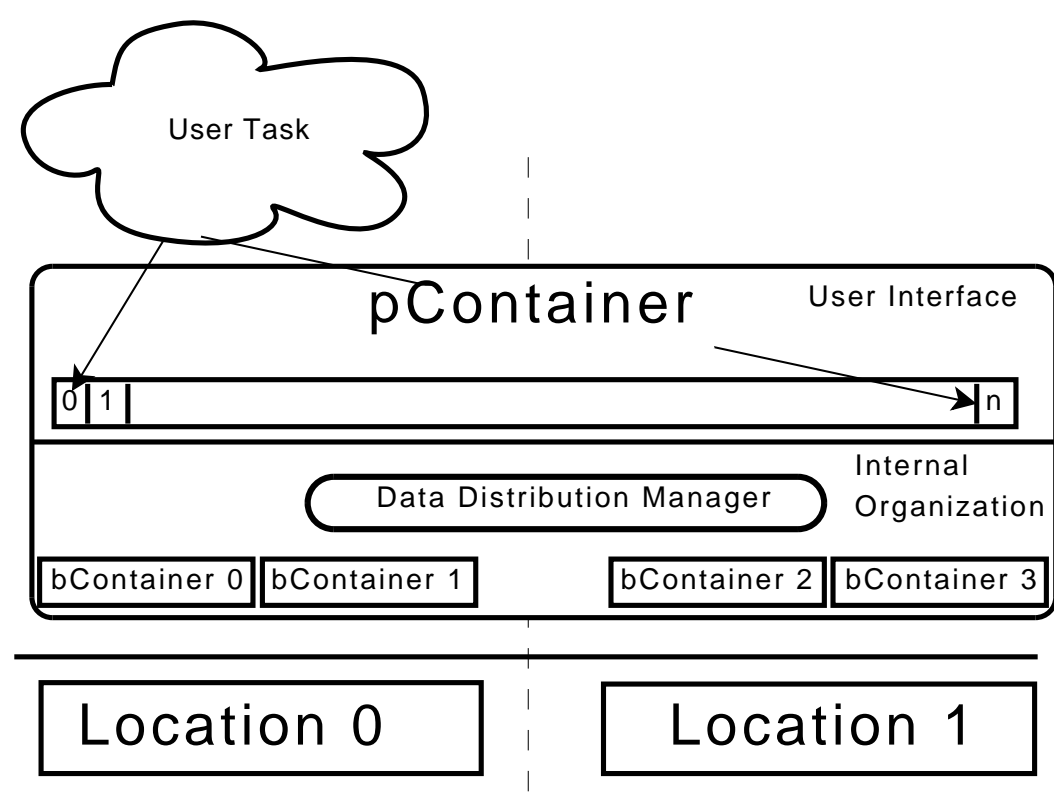

Fig. 6. Shared object view. STAPL pContainer provides a shared object view to the user. Internally it distributes the data across available locations and uses a distribution manager to find where individual elements are stored.

perform the desired operation.

The elements of a pContainer are stored in non-replicated fashion in a distributed collection of bContainers. An important function of the PCF is to provide a shared object view that relieves the programmer from managing and dealing with the distribution explicitly, unless he desires to do so. In this section, we describe how this is done.

The fundamental concept required to provide a shared object view is that each pContainer element has a unique global identifier (GID). The GID provides the shared object abstraction since all references to a given element will use the same GID. Examples of GIDs are indices for pArrays, keys for pMaps, and vertex identifiers for pGraphs. 
The PCF supports the shared object view by providing an address translation mechanism that determines where an element with a particular GID is stored (or should be stored if it does not already exist). We now briefly introduce the PCF components involved in the address translation. The set of GIDs of the elements of a pContainer is the pContainer domain $(\mathcal{D})$. A domain is partitioned into a set of nonintersecting sub-domains by a partition class, itself a distributed object that provides the map $\mathcal{F}$ from a GID to the sub-domain that contains it, i.e., a directory. There is a one-to-one correspondence between a sub-domain and a bContainer. In general, there can be multiple bContainers allocated in a location, where a location denotes a unit of a parallel machine that has a contiguous memory address space and associated execution capabilities (e.g., threads); a location may, but does not have to, be identified with a process address space. Finally, a concept called partition-mapper is used to map a sub-domain (and its corresponding bContainer) to the location where it resides, and a location-manager to manage the bContainers of a pContainer mapped to a given location.

We now describe how a pContainer method is executed using the above concepts. In Figure 7 we show a flowchart of the address resolution procedure. Given the unique GID identifying a pContainer element, the pContainer's partition is queried about the sub-domain associated with the requested GID. If the bContainer (specified by a bContainer identifier, or BCID) is not available, the partition provides information about the location (LOC) where it might be retrieved, and the process is restarted on that location. If the BCID is available and valid, then the partition-mapper receives information about the location where the bContainer resides (LID); if the operation is not local, the method is re-evaluated on that location, otherwise the location-manager provides the proper bContainer address and the operation is performed. 


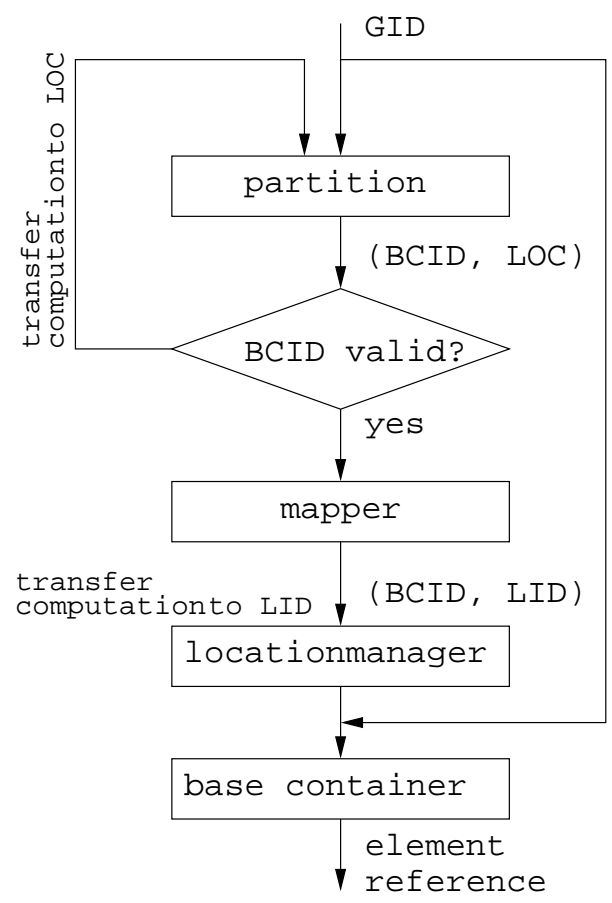

Fig. 7. pContainer address resolution. pContainer modules for performing address resolution to find the element reference corresponding to a given GID.

In dynamic pContainers, the domain may change during execution through the insertion or deletion of elements. To properly update the domain and partition information, pContainer operations are routed to the partition. In general, each method of the pContainer interface has two corresponding methods in the partition class: the where method that returns information about the sub-domain that may include the specified GID, and the execution method that actually performs the operation and updates the partition information if needed. To allow for work migration, the where method can provide an incomplete answer if the sub-domain information is not available on the location where the where method is invoked. In this case, the answer is the identifier of a location that may know the sub-domain information. This mechanism 
is referred to as method forwarding and allows the request to be migrated instead of fetching remote information for the requester. More details on partition functionality and method forwarding are included in Sections C.4 and C.6. Experimental results showing the benefits of method forwarding are included in Chapter XI, Section F.2.

In static pContainers, i.e., containers that do not support the addition and deletion of elements, the domain does not change during execution. In this case, it may be possible to optimize the address translation mechanism. In particular, if the mapping from GID to sub-domain (and hence to bContainer) has a closed form solution, then address translation is immediate and forwarding is not needed.

Next we introduce the specification and the interfaces for all modules briefly described in this section: Base Container, Location Manager, Domain, Partition, Partition Mapper and Data Distribution Manager.

\section{Base Container Interface}

The pContainer allocates a base container (bContainer) for every sub-domain defined by the partition to store the data corresponding to the sub-domain. The bContainer concept specifies a minimal interface that allows for any existing container sequential or parallel to be used as storage for the parallel container. We can not directly use the sequential container because different non standard implementations provide different interfaces for similar functionality. With the base container concept we unify sequential containers such that they can be integrated with the PCF serving as a bridge between existing data structures and PCF. The minimal interface required for a bContainer is included in Table III. 
Table III.: Base container interface.

\begin{tabular}{|c|c|}
\hline Define Types & Description \\
\hline value_type & $\begin{array}{l}\text { The element type stored in the } \\
\text { bContainer. }\end{array}$ \\
\hline reference & $\begin{array}{l}\text { The element reference type. Do not as- } \\
\text { sume that } \mathrm{T} \text { is the reference type. }\end{array}$ \\
\hline gid_type & $\begin{array}{l}\text { The gid type associated with the ele- } \\
\text { ments of the bContainer. }\end{array}$ \\
\hline bcid_type & The bContainer identifier type. \\
\hline domain_type & $\begin{array}{l}\text { The domain type associated with the } \\
\text { bContainer. }\end{array}$ \\
\hline Method & Description \\
\hline $\begin{array}{lll}\text { constructor } & \text { (domain_type*, } & \text { const } \\
\text { bcid_type\&) } & & \end{array}$ & $\begin{array}{l}\text { Construct a bContainer with the given } \\
\text { bContainer id and a given domain }\end{array}$ \\
\hline destructor(void) & $\begin{array}{l}\text { Deallocate the memory space occupied by } \\
\text { the bContainer. }\end{array}$ \\
\hline size_t size () const & $\begin{array}{l}\text { Returns the number of elements in the } \\
\text { bContainer. }\end{array}$ \\
\hline bool empty() const & $\begin{array}{l}\text { Returns true if the bContainers has zero } \\
\text { elements and false otherwise. }\end{array}$ \\
\hline void clear () & $\begin{array}{l}\text { Deallocate the space taken by the } \\
\text { bContainer elements. After clear(), } \\
\text { size() returns zero and empty () returns } \\
\text { true }\end{array}$ \\
\hline bcid_type get_bcid() & Return the bContainer identifier \\
\hline void define_type(typer \&) & $\begin{array}{l}\text { Define type for packing bContainer's } \\
\text { data }\end{array}$ \\
\hline $\begin{array}{l}\text { std::pair }<\text { size_t,size_t }>\text { mem- } \\
\text { ory_size(void) const }\end{array}$ & $\begin{array}{l}\text { Return the memory size used by } \\
\text { bContainer. The first member of the } \\
\text { pair is the memory used by data and the } \\
\text { second argument represent memory used } \\
\text { by metadata }\end{array}$ \\
\hline
\end{tabular}

The main constructor used to instantiate a bContainer takes as input a reference to the associated sub domain from the partition and a BCID. Additional methods are typical for containers and include the size() and empty(), methods to report the memory size and a method for serializing the data of a bContainer. 


\section{Location Manager Interface}

In Definition 4.1 it is shown that a pContainer $p C(\mathcal{C}, \mathcal{D}, \mathcal{F}, \mathcal{O}, \mathcal{S})$, stores elements identified by the GIDs encompassed in the domain $\mathrm{D}$, into a storage $\mathrm{S}$ that is distributed across available locations $L=\left\{L_{0}, L_{1}, \ldots L_{p-1}\right\}$. The pContainer storage consists of a collection of bContainers stored in a distributed fashion across the available locations.

A location may store a sub-set of the bContainers of a pContainer. The pContainer employs within each location a Location Manager to maintain the collection of bContainers. The complete interface is presented in Table IV. It includes methods to add and delete bContainers and methods to access individual bContainers based on their global unique BCID. The bContainers are allocated either by the pContainer or provided from outside when external storage is used. The location manager may use different optimizations for storing bContainers. For example different memory managers may be used to allocate the space required by the bContainers.

Table IV.: Location manager interface.

\begin{tabular}{|l|l|}
\hline Define Type & Description \\
\hline bcontainer_type & bContainer used to store pContainer's data. \\
\hline bcid_type & bContainer identifier type. \\
\hline iterator & $\begin{array}{l}\text { iterator type used when iterating over } \\
\text { bContainers. }\end{array}$ \\
\hline Method & Description \\
\hline default constructor & Initialize an empty location manager \\
\hline $\begin{array}{l}\text { void add_bcontainer (const } \\
\text { bcid_type\&, } \\
\text { bContainer_type*) }\end{array}$ & Add a new bContainer to the pContainer. \\
\hline size_t size() const & $\begin{array}{l}\text { Returns the number of bContainers on the cur- } \\
\text { rent location }\end{array}$ \\
\hline
\end{tabular}


Table IV continued

\begin{tabular}{|l|l|}
\hline Method & Description \\
\hline iterator begin() & Iterator pointing to the first local bContainer. \\
\hline iterator end() & $\begin{array}{l}\text { Iterator pointing one past the last local } \\
\text { bContainer. }\end{array}$ \\
\hline $\begin{array}{l}\text { bcontainer_type* } \\
\text { get_bcontainer(const } \\
\text { bcid_type\&) }\end{array}$ & $\begin{array}{l}\text { Returns a pointer to a given bContainer specified } \\
\text { as argument }\end{array}$ \\
\hline void clear(void) & $\begin{array}{l}\text { Deletes the bContainers and their corresponding } \\
\text { memory }\end{array}$ \\
\hline $\begin{array}{l}\text { pair<size_t,size_t }> \\
\text { memory_size(void) const }\end{array}$ & $\begin{array}{l}\text { Compute the memory size for location manager } \\
\text { meta data and data }\end{array}$ \\
\hline
\end{tabular}

\section{Domain Interface}

Derived from its definition in Chapter IV, Section B.2, a pContainer domain specifies the GID type of the domain, test if a specific GID belongs to the domain, compute its cardinality if finite and provide methods to enumerate its GIDs. The framework most commonly uses ordered domains to describe the set of GIDs of a pContainer and the order among the elements corresponding to the GIDs. An ordered domain implementation supports the additional functionality described next and is required to implement the interface from Table V.

1. Specify the first and the last element of the domain according to a total order $R$. The first element belongs to the domain but the last element does not belong to the domain.

$\forall x \in O D,($ firstRx $)$ and (xRlast) and not (lastRx).

This is a requirement that provides us compatibility with $\mathrm{C}++\mathrm{STL}$, where the end of a range is a convention such that all elements in the range are less than it. 
2. Compare two GIDs according to $R$ (e.g., gid $_{1} R g i d_{2}$ is either true or false).

Table V.: Ordered domain interface.

\begin{tabular}{|l|l|}
\hline Define Type & Description \\
\hline gid_type & The global unique identifier type \\
\hline Method & Description \\
\hline gid_type get_first_gid() const & The first gid of the domain \\
\hline gid_type get_last_gid() const & $\begin{array}{l}\text { The last gid of the domain; a convention } \\
\text { with the property that every other gid of } \\
\text { the domain will be less than it }\end{array}$ \\
\hline bool contains_gid (gid_type_gid) const & $\begin{array}{l}\text { Returns true or false depending if the do- } \\
\text { main is part of the domain }\end{array}$ \\
\hline $\begin{array}{l}\text { bool compare_less_gids (gid_type, } \\
\text { gid_type) const }\end{array}$ & Compare for 'less then' two GIDs \\
\hline gid_type get_invalid_gid() const & $\begin{array}{l}\text { Required to represent NULL/invalid iter- } \\
\text { ators in the pView }\end{array}$ \\
\hline
\end{tabular}

A Finite Ordered Domain (FOD) extends the ordered domain with the following functionality and the interface included in Table VI.

1. Return the number of elements in the domain (e.g., cardinality).

2. The next gid of a gid defined as next :FOD $\rightarrow$ FOD, next $(x)=y$, such that $x R y$ and there is no other $z \in F O D, x R z$ and $z R y$

3. The previous gid of a gid defined as prev :FOD $\rightarrow$ FOD, prev $(x)=$ $y$, such that $x R y$ and there is no other $z \in F O D, y R z$ and $z R x$

4. The $n^{\text {th }}$ gid following a gid defined as advance ${ }_{n}: F O D \rightarrow F O D$, $_{\text {advance }}(x)=$ $y$, such that 
$y=n \operatorname{ext}(\ldots n \operatorname{ext}(n \operatorname{ext}(x)) \ldots)$, ntimes; apply next $n$ times starting with $x$.

5. An unique enumeration imposed by $R$. Starting from the first element $e_{0}$ there is a unique enumeration $e_{0} R e_{1} R \ldots R e_{n-1}$ that contains all elements in FOD.

6. An offset of a gid within the unique enumeration specified by $R$ which is defined as

offset $: F O D \rightarrow N($ natural numbers $)$, offset $(x)=$ niff advance $_{n}($ first $)=$ $x$.

Table VI.: Finite ordered domain interface.

\begin{tabular}{|l|l|}
\hline Define Type & Description \\
\hline gid_type & The global unique identifier type \\
\hline Method & Description \\
\hline size_t size (void) const & The size of the domain \\
\hline gid_type get_next_gid (gid_type) const & $\begin{array}{l}\text { The next gid of the argument according } \\
\text { to the domain ordering relation }\end{array}$ \\
\hline gid_type get_prev_gid (gid_type) const & The previous gid of the argument \\
\hline $\begin{array}{l}\text { gid_type advance (gid_type, size_t n) } \\
\text { const }\end{array}$ & The nth gid after the current one \\
\hline size_t offset (gid_type) const & $\begin{array}{l}\text { The offset of the gid in the linearization } \\
\text { of the domain }\end{array}$ \\
\hline
\end{tabular}

\section{Partition Interface}

In Chapter IV, Sections B.4 and B.5 we introduced the main functionality of the partition. It specifies a decomposition of a domain into a collection of sub-domains. The partition is one of the main functional modules of a pContainer and we envision that this will be the most common mechanism used to customize the behavior of 
the pContainer. In addition to specifying a collection of sub-domains the partition will provide the information regarding the BCID associated with a given GID, and will specify the behavior of individual pContainer methods.

For a dynamic pContainer as described later in Section D.3 the partition will specify the bContainer where a new element will be added and the specific actions that need to happen when the element is added to an individual bContainer. For this reason, we decided that every pContainer method will have two corresponding methods at the partition level describing the bContainer where the method will be executed and how it will be executed. In Section D we include details on the additional interface requirements for the partitions of various pContainer specializations. When atomicity is provided by the framework the partition will additionally specify the locking modes for each individual method in the pContainer interface. This will be discussed in more detail in Chapter VI.

Derived from the partition description and properties introduced in this section we designed the interface included in Table VII.

Table VII.: Partition base interface.

\begin{tabular}{|l|l|}
\hline Define Type & Description \\
\hline domain_type & domain type \\
\hline bcid_type & sub domain identifier type \\
\hline $\begin{array}{l}\text { bcid_type get_info(const GID\&) } \\
\text { const }\end{array}$ & $\begin{array}{l}\text { Returns the bContainer identifier associated with } \\
\text { input GID. }\end{array}$ \\
\hline $\begin{array}{l}\text { const bcids_info_type\& } \\
\text { get_cids_info(void) const }\end{array}$ & $\begin{array}{l}\text { Returns a structure with the information about } \\
\text { the order among BCIDs. }\end{array}$ \\
\hline size_t size() const & Returns the total number of sub domains \\
\hline $\begin{array}{l}\text { void get_sub_domains_sizes( } \\
\text { std::vector }<\text { size_t }>\&) \text { const }\end{array}$ & Return the sizes of the sub domains \\
\hline $\begin{array}{l}\text { void set_domain(domain_type* }) \\
\text { Sets the domain of the partition. The partition } \\
\text { initializes its sub domains to reflect a partition of } \\
\text { the input domain. }\end{array}$ \\
\hline
\end{tabular}


Table VII continued

\begin{tabular}{|l|l|}
\hline Method & Description \\
\hline $\begin{array}{l}\text { domain_type* get_domain() } \\
\text { const }\end{array}$ & Get the domain of the partition. \\
\hline $\begin{array}{l}\text { domain_type* } \\
\text { get_sub_domain(const } \\
\text { bcid_type\&) }\end{array}$ & Get a certain sub domain. \\
\hline $\begin{array}{l}\text { const } \\
\text { std::vector<domain_type* } \\
\text { get_sub_domains() const }\end{array}$ & Get the sub domains of the partition. \\
\hline $\begin{array}{l}\text { void set_partition_mapper (par- } \\
\text { tition_mapper_type*_pm) }\end{array}$ & Set a reference to the associated partition mapper. \\
\hline $\begin{array}{l}\text { partition_mapper_type* } \\
\text { get_partition_mapper(void) }\end{array}$ & Get a reference to the associated partition mapper. \\
\hline size_t memory_size() const & $\begin{array}{l}\text { Compute the memory used. Counted as part of } \\
\text { the pContainer metadata. }\end{array}$ \\
\hline
\end{tabular}

Table VIII.: Ordered partition interface.

\begin{tabular}{|l|l|}
\hline Define Type & Description \\
\hline bcid_type & bContainer identifier type \\
\hline Method & Description \\
\hline bcid_type get_first() & The identifier of the first bContainer. \\
\hline bcid_type get_last() & $\begin{array}{l}\text { Last identifier. } \\
\text { that get_next (last valid bContainer } \\
\text { identifier) returns get_last() }\end{array}$ \\
\hline bcid_type get_next(bcid_type) & $\begin{array}{l}\text { Computes the identifier of the bContainer follow- } \\
\text { ing the one given as argument, according to the } \\
\text { relation order the ordered partition implements }\end{array}$ \\
\hline bcid_type get_prev(bcid_type) & $\begin{array}{l}\text { Computes the identifier of the bContainer before } \\
\text { the one given as argument }\end{array}$ \\
\hline
\end{tabular}

The ordered partition is another concept of the PCF and it requires users to 
provide, as a nested data type, a class encapsulating the order among sub domains. This concept is referred to as bcids_info and has the interface included in Table VIII.

\section{Partition Mapper Interface}

In the PCF a unique bContainer identifier (BCID) is associated with every subdomain of a partition. The partition-mapper of a pContainer provides the mapping from the set of sub domain identifiers (BCIDs) to a set of locations. The pContainer will use the partition mapper to decide the locations where individual bContainers will be allocated and an interface to find where a specific BCID has been mapped. The complete interface is included in Table IX.

Table IX.: Partition mapper interface.

\begin{tabular}{|l|l|}
\hline Define Type & Description \\
\hline bcid_type & bContainer identifier type \\
\hline location_type & $\begin{array}{l}\text { Location identifier type; a type convertible to } \\
\text { armi::location_type }\end{array}$ \\
\hline partition_mapper() & $\begin{array}{l}\text { Default constructor; the mapper is not initialized; } \\
\text { init can be used afterward }\end{array}$ \\
\hline $\begin{array}{l}\text { bool is_local(const bcid_type\& }) \\
\text { const }\end{array}$ & $\begin{array}{l}\text { Returns true or false depending if the argument } \\
\text { BCID is local or not }\end{array}$ \\
\hline $\begin{array}{l}\text { const std::vector<bcid_type }>\& \\
\text { get_local_cids() const }\end{array}$ & Returns the list of local allocated BCIDs. \\
\hline $\begin{array}{l}\text { location_type map(const } \\
\text { bcid_type\& } \\
\text { const }\end{array}$ & $\begin{array}{l}\text { Returns the location where the sub domain iden- } \\
\text { tifier passed as argument may live }\end{array}$ \\
\hline void init(const cids_info_type\&) & $\begin{array}{l}\text { Initialize the partition mapper with information } \\
\text { about the list of BCIDs passed as argument }\end{array}$ \\
\hline size_t get_num_bcontainers() & $\begin{array}{l}\text { Returns the total number of bContainers man- } \\
\text { aged by the current mapper }\end{array}$ \\
\hline size_t memory_size(void) const & $\begin{array}{l}\text { Returns the memory size occupied by this object. } \\
\text { It will be counted by the pContainer as part of } \\
\text { the metadata memory usage }\end{array}$ \\
\hline
\end{tabular}


The PCF provides a set of partition mappers that are briefly introduced next. Assuming the sub-domain identifiers are from 0 to $m-1$ and the location identifiers are from 0 to $L-1$. The cyclic mapper, for which sub-domains are distributed cyclically among locations; blockedmapper, where $m / L$ consecutive sub-domains are mapped in a single location and general mapper that can arbitrary map any sub-domain to any location.

\section{Data Distribution Manager}

The data_distribution manager base class is responsible for managing the pContainer partition and partition mapper. All pContainer methods that deal with elements are forwarded to the data distribution manager. This class uses the partition and the partition mapper to determine the locations and the bContainers where the method will be executed finally.

As shown in Chapter VI, to simplify the pContainer developers effort while interacting with the partition, the partition mapper, and thread safety management, the data-distribution-manager provides a skeleton for any element-wise method that users can customize by providing appropriate functors. The generic method execution support is encapsulated within a set of methods called invoke which are shown in Figure 8. The actions performed inside invoke require cooperation from all previously introduced pContainer modules. The method receives as input a unique method identifier and two functors : FunctorWhere and FunctorAction. Both functors are redirections to the appropriate methods in the partition. The first action performed by invoke is to query the partition for the BCID of the bContainer where the method needs to be invoked (Figure 8, line 5). The partition returns a bContainer info structure that either contains the exact bContainer identifier or a new location where the method needs to be forwarded to be further processed (Figure 8, line 9). If 


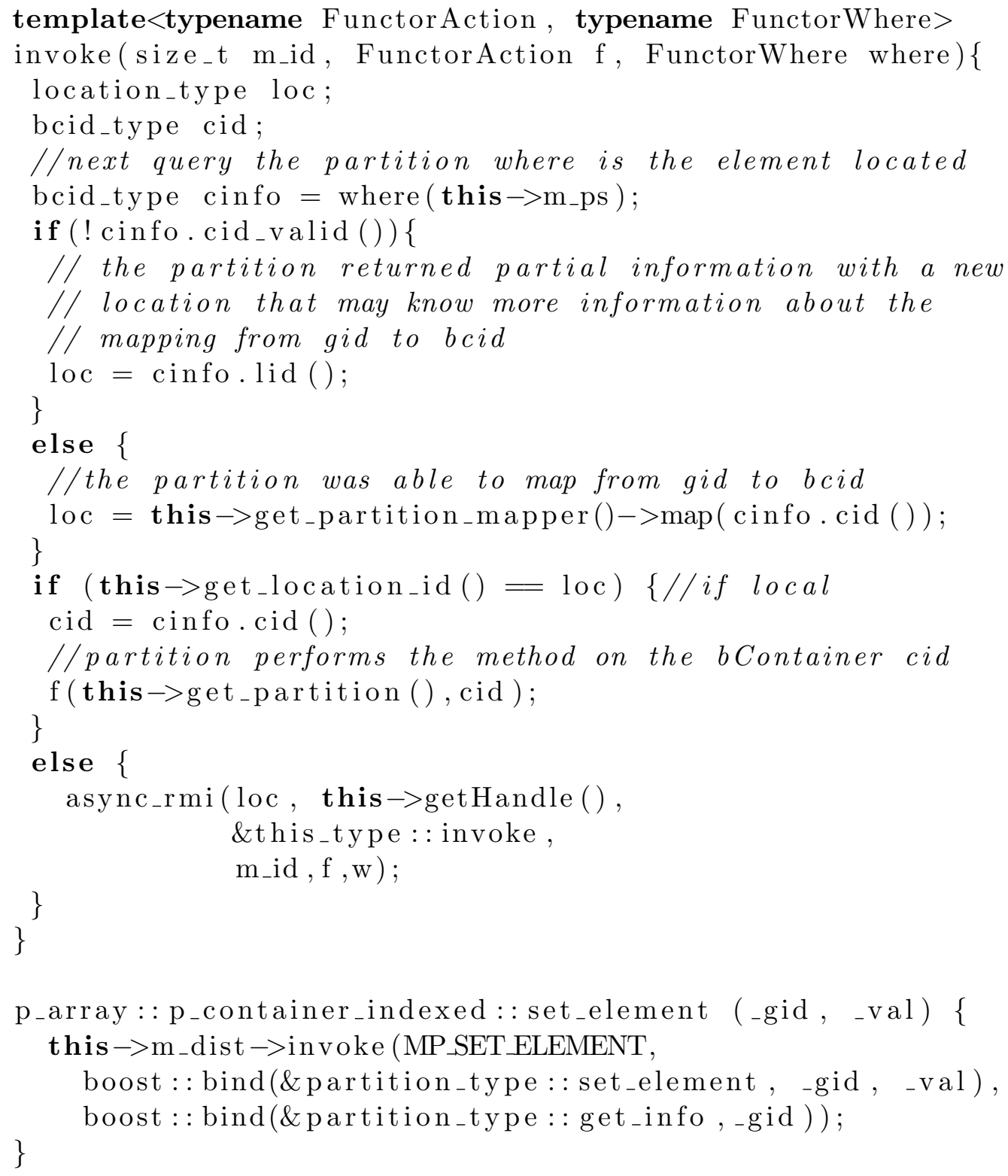

Fig. 8. The invoke method of the data distribution manager. 
the exact BCID is returned by the partition, then the pContainer uses the partition mapper to identify the location where the method needs to be executed or forwarded (Figure 8, line 13). If the identified location is the current one, then the partition will be asked to perform the FunctorAction on the corresponding bContainer (Figure 8 , line 17). If the location is a remote one, than the method will be forwarded and re-executed on that location (Figure 8, line 20).

With this mechanism the pContainer can implement the shared object view. The methods are forwarded and executed on corresponding locations according to the policies specified in the partition class. In Figure 8, starting with line 26, we show the implementation of the pArray set_element method as a simple redirection to the distribution manager invoke method with the FunctorWhere querying the partition get_info and the FunctorAction invoking set_element method of the partition for indexed pContainer.

The invoke methods perform additional actions related to the atomicity of execution that will be discussed in Chapter VI. The data-distribution-manager interface is included in Table X. 
Table X.: Data distribution manager interface.

\begin{tabular}{|c|c|}
\hline Define Types & Description \\
\hline domain_type & The partition domain type. \\
\hline gid_type & The partition GID. \\
\hline bcid_type & The partition BCID. \\
\hline partition_mapper_type & Partition mapper type \\
\hline location_type & The location type \\
\hline partition_type & The partition type \\
\hline location_manager_type & The location manager type \\
\hline ths_manager_type & The thread safety manager type \\
\hline Method & Description \\
\hline data_distribution_base() & $\begin{array}{l}\text { Default constructor; the distribution in- } \\
\text { formation is uninitialized }\end{array}$ \\
\hline $\begin{array}{l}\text { data_distribution_base(domain_type\& } \\
\text { domain, partition_type p) }\end{array}$ & $\begin{array}{l}\text { Initialize the data distribution informa- } \\
\text { tion based on the input domain and par- } \\
\text { tition; the partition mapper will be allo- } \\
\text { cated based on the template argument }\end{array}$ \\
\hline data_distribution_base() & $\begin{array}{l}\text { Destructor in charge of calling the de- } \\
\text { structor of the partition and partition } \\
\text { mapper }\end{array}$ \\
\hline void clear() & $\begin{array}{l}\text { Deallocate the partition and the partition } \\
\text { mapper }\end{array}$ \\
\hline $\begin{array}{l}\text { bcid_type get_info(const gid_type\&_gid) } \\
\text { const }\end{array}$ & $\begin{array}{l}\text { Returns the sub domains that contains } \\
\text { the input GID. }\end{array}$ \\
\hline bool is_local(const gid_type\&_gid) const & $\begin{array}{l}\text { Returns true or false if the GID argument } \\
\text { is mapped on the current location }\end{array}$ \\
\hline $\begin{array}{ll}\text { location_type } & \text { lookup(const } \\
\text { gid_type\&_gid) const } & \end{array}$ & $\begin{array}{l}\text { Returns the location that owns or that } \\
\text { may have more information about the } \\
\text { GID }\end{array}$ \\
\hline partition_type* get_partition() & Returns a pointer to the partition. \\
\hline $\begin{array}{l}\text { partition_mapper_type* } \\
\text { get_partition_mapper }()\end{array}$ & $\begin{array}{l}\text { Returns a pointer to the partition map- } \\
\text { per. }\end{array}$ \\
\hline $\begin{array}{l}\text { template }<\text { typename FunctorAction, } \\
\text { typename FunctorWhere }> \\
\text { void invoke(size_t m_id, const FunctorAc- } \\
\text { tion\& f, const FunctorWhere\& w) }\end{array}$ & $\begin{array}{l}\text { Support for asynchronous method execu- } \\
\text { tion }\end{array}$ \\
\hline $\begin{array}{l}\text { typename FunctorAction::result_type in- } \\
\text { voke_ret(size_t m_id, const FunctorAc- } \\
\text { tion\& f, const FunctorWhere\& w) }\end{array}$ & $\begin{array}{l}\text { Support for synchronous method execu- } \\
\text { tion }\end{array}$ \\
\hline $\begin{array}{l}\text { typename FunctorAction::result_type in- } \\
\text { voke_opaque_ret(size_t m_id, const Func- } \\
\text { torAction\& f, const FunctorWhere\& w) }\end{array}$ & Support for split phase method execution \\
\hline
\end{tabular}


Table X continued

\begin{tabular}{|l|l|}
\hline Method & Description \\
\hline size_t memory_size(void) const & $\begin{array}{l}\text { Memory used by this class and its data } \\
\text { members (partition, partition mapper); } \\
\text { reported as metadata }\end{array}$ \\
\hline
\end{tabular}

The pContainer uses the data distribution (partition, partition mapping), the location manager, and the bContainer, to determine the complete location information (location, memory reference within location) where the data element corresponding to a certain GID is allocated. The data distribution manager, the location manager, and the bContainer are the modules in our framework that allow the pContainer to provide a shared memory view to the user.

\section{Specification for pContainer Framework Concepts}

In this section we introduce the complete interface of the base pContainer classes introduced in Section A and depicted in Figure 5. Each of these concepts will have associated interfaces and requirements for bContainers, Domains, Partitions, Partition Mappings and Location Managers which are discussed in the following sections.

\section{1. pContainer Base}

All STAPL pContainers derive from p_container_base class. This class is in charge of storing the data using a location-manager and data distribution information using a data-distribution-manager. It provides a simple interface to initialize the pContainer based on the traits class provided as a template argument, and a domain and partition instance. The complete interface is described in Table XI and includes constructors, copy constructors that copy both data and metadata, and methods 
to retrieve references to the location-manager and data-distribution-manager. The type of the domain, partition, partition mapper, location-manager are passed to this base class using the template traits class. Users will be able to customize the behavior of this class by passing proper traits. This process will be exemplified in Section H.

Table XI.: Base pContainer interface.

\begin{tabular}{|l|l|}
\hline Template Arguments & Description \\
\hline Traits & pContainer traits; \\
\hline Define type & Description \\
\hline partition & pContainer partition; Specified in the traits. \\
\hline domain & $\begin{array}{l}\text { pContainer domain; Specified in the partition } \\
\text { type. }\end{array}$ \\
\hline location_manager_type & $\begin{array}{l}\text { pContainer location manager; Specified in the } \\
\text { traits. }\end{array}$ \\
\hline distribution_type & $\begin{array}{l}\text { pContainer distribution manager; Specified in the } \\
\text { traits. }\end{array}$ \\
\hline Method & Description \\
\hline constructor & $\begin{array}{l}\text { Collective Operation. Default constructor, regis- } \\
\text { ters the pContainer with RTS. }\end{array}$ \\
\hline $\begin{array}{l}\text { constructor( } \\
\text { p_container_base\& other) }\end{array}$ & $\begin{array}{l}\text { Collective Operation. Registers the pContainer } \\
\text { with RTS and copy the content of the other. }\end{array}$ \\
\hline $\begin{array}{l}\text { void init(domain*, } \\
\text { partition*) }\end{array}$ & $\begin{array}{l}\text { Initialize based on a domain and partition. The } \\
\text { partition is used to define the sub domains and } \\
\text { implicitly the bContainers of the pContainer. }\end{array}$ \\
\hline $\begin{array}{l}\text { location_manager_type* } \\
\text { get_location_manager( })\end{array}$ & $\begin{array}{l}\text { Returns a pointer to the pContainer location } \\
\text { manager }\end{array}$ \\
\hline $\begin{array}{l}\text { distribution_type* } \\
\text { get_distribution() }\end{array}$ & Returns a pointer to the data distribution manager \\
\hline
\end{tabular}

All STAPL pContainers are pObjects, which means that they need to be allocated in an SPMD style on all locations where the pContainer will distribute its 
data. The pContainer will have a representative on all these locations and the union of all pContainer representatives makes the overall pContainer. Having access to a pContainer representative is equivalent to having access to the whole pContainer. According to these requirements, the constructors are collective operations and they are responsible for registering the pContainer with the RTS. The registration will happen in the base class of the p_container_base, p_object. The registration returns a handle object that is stored and used during the pContainer's live time to perform remote method invocations across different locations.

The init method is in charge of the initial setup of the pContainer classes. It takes as argument a domain and a partition. It will ask first the partition to provide a decomposition of the given domain, followed by a query of the partition mapper to decide which of the sub-domains will be mapped to which individual locations. This process happens simultaneously on all locations and subsequently all locations will allocate the bContainers for the local allocated sub domains. The bContainers are then added to the location manager that will further administer them.

All STAPL pContainers are designed to report their memory usage and this is provided by the memory_size method. This is a collective operation which will return a pair containing the metadata size in the first field and the data size in the second field. The p_container_base achieves this by recursively invoking the method size on the data-distribution-manager and location-manager. The size is reported in bytes.

In the following sections we describe the extensions and specializations of the base concepts introduced for the different pContainer specializations proposed in our taxonomy. 


\section{Static pContainer}

The size of a static pContainers is fixed when the pContainer is declared and will not change afterward. All interfaces described for the base classes will be available for static pContainers. The property that the number of elements is fixed allows for more efficient implementations of domains, partitions and pViews to be used. For example partitions and pViews based on closed form solutions can be used with this type of container.

Table XII.: Static pContainer interface.

\begin{tabular}{|l|l|}
\hline Template Arguments & Description \\
\hline Traits & pContainer traits; \\
\hline Method & Description \\
\hline size_t local_size() const & Returns the local size of the pContainer. \\
\hline size_t size() const & Returns the size of the pContainer. \\
\hline bool local_empty() const & Check if all the local bcontainers are empty. \\
\hline bool empty() const & $\begin{array}{l}\text { Check if the pContainer is empty; return true if the } \\
\text { pContainer is empty and false otherwise. }\end{array}$ \\
\hline $\begin{array}{l}\text { template<class Functor }> \\
\text { typename Functor::result_type } \\
\text { apply_get(gid_type i, Functor f) }\end{array}$ & $\begin{array}{l}\text { Apply a functor f to the data corresponding to the } \\
\text { GID; The functor has a return type. }\end{array}$ \\
\hline $\begin{array}{l}\text { template<class Functor> } \\
\text { void apply_set(gid_type i, Functor } \\
\text { f) }\end{array}$ & $\begin{array}{l}\text { Apply a functor f to the data corresponding to the } \\
\text { GID; The functor does not have a return type. }\end{array}$ \\
\hline iterator begin() const & Iterator to the first element of the pContainer. \\
\hline iterator end() const & Iterator pointing one past last valid element. \\
\hline bool is_local(gid_type gid) const & $\begin{array}{l}\text { Returns true or false if the argument GID is local or } \\
\text { not. }\end{array}$ \\
\hline $\begin{array}{l}\text { location_type lookup(gid_type } \\
\text { gid) const }\end{array}$ & $\begin{array}{l}\text { Returns the location where the given GID may be } \\
\text { found. }\end{array}$ \\
\hline $\begin{array}{l}\text { bool is_local(gid_type } \\
\text { cid_type\& bcid) const }\end{array}$ & $\begin{array}{l}\text { Returns true or false if the argument GID is local or } \\
\text { not. }\end{array}$ \\
\hline
\end{tabular}


The additional interface that a static pContainer provides is included in Table XII. It includes query methods for different properties such as size() and empty() methods to obtain references to the first or last element of the pContainer, and references to an element with a given GID.

\section{Dynamic pContainer}

A dynamic pContainer allows elements to be added and deleted from a pContainer. We include in Table XIII the additional interface a dynamic pContainer supports.

Table XIII.: Dynamic pContainer interface.

\begin{tabular}{|l|l|}
\hline Template Arguments & Description \\
\hline Traits & pContainer traits; \\
\hline Method & Description \\
\hline void clear() & $\begin{array}{l}\text { Equivalent to remove all elements; The distribution } \\
\text { and location manager remain valid }\end{array}$ \\
\hline $\begin{array}{l}\text { cid_type } \\
\text { add_bcontainer(bcontainer_type* } \\
\text { c) }\end{array}$ & $\begin{array}{l}\text { Add a bContainer allocated dynamic outside; the } \\
\text { pContainer will just use it without any copying in- } \\
\text { volved }\end{array}$ \\
\hline $\begin{array}{l}\text { cid_type } \\
\text { delete_bcontainer(bcontainer_type* } \\
\text { c) }\end{array}$ & \begin{tabular}{l} 
Delete the requested bContainer. \\
\hline
\end{tabular} \\
\hline
\end{tabular}

\section{Indexed pContainer}

Containers in this category provide an interface to access the elements based on their index. The domains associated with the containers in this category are derived from Cartesian domains or compositions of Cartesian domains.

The indexed pContainer inherits either from a static or dynamic pContainer 
and this is specified in the traits class. The additional interface supported by an indexed pContainer is included in Table XIV.

Table XIV.: Indexed pContainer interface.

\begin{tabular}{|l|l|}
\hline Methods & Description \\
\hline $\begin{array}{l}\text { void set_element(const gid_typ\& } \\
\text { gid, const value_type\& val) }\end{array}$ & $\begin{array}{l}\text { Set the value of an element associated with a certain } \\
\text { gid. }\end{array}$ \\
\hline $\begin{array}{l}\text { value_type get_element(const } \\
\text { gid_type\& gid) const }\end{array}$ & $\begin{array}{l}\text { Get the value of an element associated with a certain } \\
\text { gid }\end{array}$ \\
\hline $\begin{array}{l}\text { pc_future<value_type }> \\
\text { split_phase_get_element(const } \\
\text { gid_type\& gid) const }\end{array}$ & $\begin{array}{l}\text { split phase get element (two phase get element); It } \\
\text { returns a future that can be queried if the value is } \\
\text { available or not }\end{array}$ \\
\hline reference operator[](gid_type gid) & $\begin{array}{l}\text { Returns a reference to the element corresponding to } \\
\text { the GID; }\end{array}$ \\
\hline $\begin{array}{l}\text { value_type operator[](gid_type } \\
\text { gid) const }\end{array}$ & Returns the element \\
\hline
\end{tabular}

Corresponding to the element-wise interface introduced (e.g., set_element, get_element, split_phase_get_element) the partitions of indexed pContainers require the additional interface included in Table XV. The new interface is used by the pContainer indexed as the FunctorAction. For the FunctorWhere the generic get_info method of the partition base class is used as exemplified in Figure 9. 


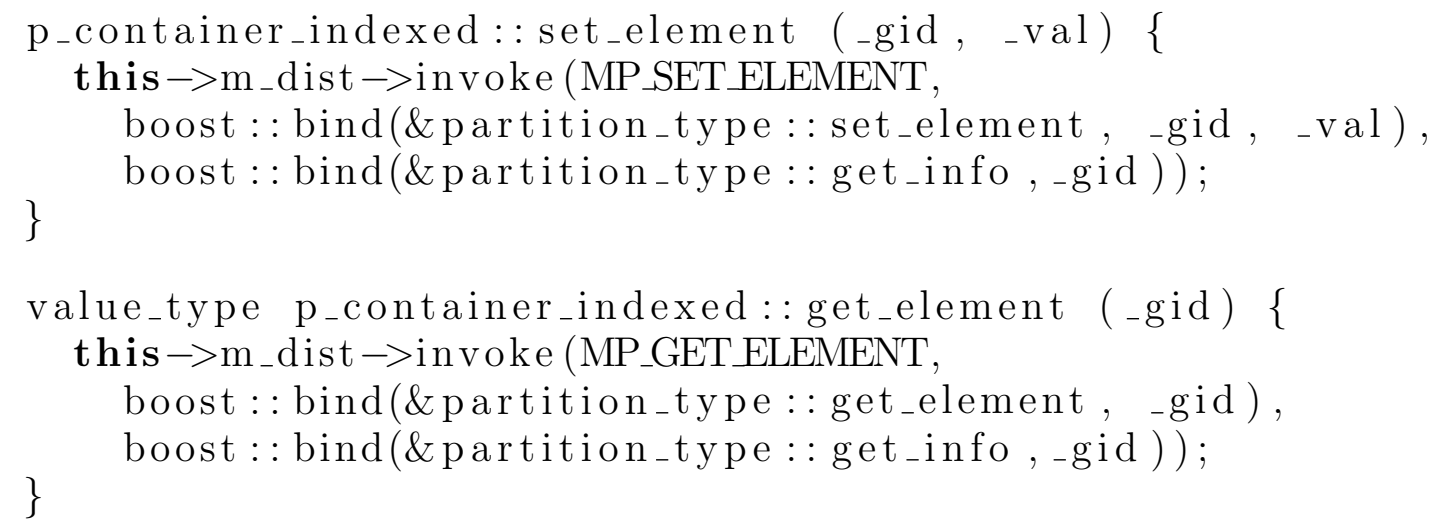

Fig. 9. The pContainer indexed method implementation.

Table XV.: Indexed partition interface.

\begin{tabular}{|l|l|}
\hline Methods & Description \\
\hline $\begin{array}{l}\text { void set_element(const gid_typ\& } \\
\text { gid, const value_type\& val, const } \\
\text { bcid_type\& bcid) }\end{array}$ & $\begin{array}{l}\text { Set the value of an element associated with a certain } \\
\text { gid in the specified bContainer. }\end{array}$ \\
\hline $\begin{array}{l}\text { value_type get_element(const } \\
\text { gid_type\& gid, const bcid_type\& } \\
\text { bcid) const }\end{array}$ & $\begin{array}{l}\text { Get the value of an element associated with a certain } \\
\text { gid }\end{array}$ \\
\hline
\end{tabular}

The domains used with indexed partitions need to implement the finite ordered domain interface as specified in Chapter IV, Section B.3. The framework provides default implementations for one and two dimensional indexed pContainers that are used by pArray, pVector, pMatrix. 
Indexed Partitions:

- partition_balanced: used by pArray. For a given domain of size $N$ it will create $P$ sub-domains, each of size $N / P$. If $N<P$ then there will be $N$ sub domains of size 1 ;

- partition_blocked : used by pArray. For a given domain of size $N$ and a block size $B S$ there will be $N / B S$ sub domains created, each of size $B S$

- partition_blocked_explicit: used by pArray. The constructors will accept an explicit decomposition of a domain in sub-domains of arbitrary sizes.

- pv_unbalanced_partition: used by pVector. It is initially constructed similar to balanced_partition of the pArray. Subsequent insert or delete operations may lead to unbalanced blocked partitions.

- p_matrix_partition: used by pMatrix. Allows user to specify block or block cyclic decompositions, with row or column wise decompositions.

\section{User-Partition Interaction}

Advanced users will interact with the partition to specify the granularity of data for different computations and to control how data will map on the machine. Creating a new partition object will be performed by invoking the appropriate constructors. For example, for static array-like data structures the users can choose from among the following partition strategies:

Examples: We assume a domain $\mathrm{D}=[1 . .10]$.

1. partition_balanced(domain, $2 / *$ num_subdomains*/);

$$
\mathrm{P}=\{0 . .5,6 . .10\}
$$


2. partition_blocked(domain, 3/*block size*/);

$$
\mathrm{P}=\{0 . .2,3 . .5,6 . .8,9 . .10\}
$$

3. partition_block_cyclic(domain, 2, BLOCK_CYCLIC (3));

$$
\mathrm{P}=\{\{0,1,2,, 6,7,8\}\{3,4,5,, 9,10\}\} / / \text { two domains, cyclic, group size } 3
$$

4. partition_block_cyclic(domain, 2, BLOCK_CYCLIC(1));

$$
\mathrm{P}=\{\{0,2,4,6,8,10\}\{1,3,5,7,9\}\} / / \text { two domains, cyclic, block } 1
$$

5. partition_blocked_explicit(domain, $\operatorname{BLOCK}(\mathrm{v}\{3,4,4\}))$;

$$
\mathrm{P}=\{0 . .2,3 . .6,7 . .10\}
$$

The above examples can be correspondingly extended to multi-dimensional domains such as 2D or 3D arrays.

\section{Associative pContainer}

Containers in this category are dynamic pContainers that have associated two data types: Key and Value. The interface supported by an associative container extends the interface provided by the base and dynamic to accommodate the fact that we have key/value pairs and it is included in Table XVI.

Table XVI.: Associative pContainer interface.

\begin{tabular}{|l|l|}
\hline Template Arguments & Description \\
\hline Traits & pContainer traits; \\
\hline Key & Key type \\
\hline Value & Value type \\
\hline Methods & Description \\
\hline $\begin{array}{l}\text { reference operator[](const } \\
\text { gid_type\& gid) }\end{array}$ & $\begin{array}{l}\text { Returns a reference to the element corresponding to } \\
\text { the GID }\end{array}$ \\
\hline $\begin{array}{l}\text { void erase_async(const key_type\& } \\
\text { key) }\end{array}$ & Erases key asynchronously \\
\hline
\end{tabular}




\begin{tabular}{|l|l|}
\hline void clear() & $\begin{array}{l}\text { Clears the pContainer; equivalent to } \\
\text { erase(begin,end) }\end{array}$ \\
\hline $\begin{array}{l}\text { iterator find(const key_type\& } \\
\text { key) }\end{array}$ & $\begin{array}{l}\text { Returns an iterator pointing to an element identified } \\
\text { by its key. If the key does not exist the pContainer's } \\
\text { end is returned. }\end{array}$ \\
\hline $\begin{array}{l}\text { pair<value_type,bool }> \\
\text { find_val(const key_type\& key) } \\
\text { const }\end{array}$ & $\begin{array}{l}\text { Find value_type corresponding to key_type. The } \\
\text { boolean of the pairs signals the fact that the ar- } \\
\text { gument key exists in the pContainer (true) or not } \\
\text { (false) }\end{array}$ \\
\hline $\begin{array}{l}\text { pc_future<value_type> } \\
\text { split_phase_find(const key_type\& } \\
\text { key) const }\end{array}$ & $\begin{array}{l}\text { Split phase find value corresponding to the key; It } \\
\text { returns a future that can be queried if the value is } \\
\text { available or not }\end{array}$ \\
\hline
\end{tabular}

\section{Relational pContainer}

Containers in this category store elements (e.g., vertices in a graph) and relationships between elements (e.g., edges). The interface extends the base classes with methods to specify relationships between elements and it is included in Table XVII.

Table XVII.: Relational pContainer interface.

\begin{tabular}{|l|l|}
\hline Template Arguments & Description \\
\hline Traits & pContainer traits; \\
\hline Define Type & Description \\
\hline vertex_property & Vertex property type \\
\hline vertex_descriptor & Vertex descriptor type \\
\hline edge_descriptor & Edge descriptor type \\
\hline edge_property & Edge property type \\
\hline vertex_iterator & Vertex iterator type \\
\hline const_vertex_iterator & Const vertex Iterator \\
\hline adj_edge_iterator & Adjacency edge iterator \\
\hline const_adj_edge_iterator & const adjacency edge iterator \\
\hline Method & Description \\
\hline
\end{tabular}


Table XVII continued

\begin{tabular}{|c|c|}
\hline Method & Description \\
\hline gid_type add_vertex(void) & $\begin{array}{l}\text { Add a new vertex into the graph; returns the vertex } \\
\text { descriptor. }\end{array}$ \\
\hline $\begin{array}{l}\text { gid_type add_vertex(const ver- } \\
\text { tex_property\& vp) }\end{array}$ & $\begin{array}{l}\text { Add a new vertex into the graph with the specified } \\
\text { property. }\end{array}$ \\
\hline $\begin{array}{l}\text { void add_vertex(const gid_type\& } \\
\text { gid, const vertex_property\& vp) }\end{array}$ & $\begin{array}{l}\text { Add a vertex with value val and vertex descriptor } \\
\text { gid. }\end{array}$ \\
\hline $\begin{array}{l}\text { void delete_vertex(const } \\
\text { gid_type\&_vd) }\end{array}$ & $\begin{array}{l}\text { The following method is not a transaction; Deleting } \\
\text { edges and vertices are atomic but the whole method } \\
\text { is not atomic since the execution of this method may } \\
\text { be done across different locations. }\end{array}$ \\
\hline $\begin{array}{lrr}\text { edge_descriptor } & \text { add_edge(const } \\
\text { edge_descriptor\& } & \text { ed, } r \text { const } \\
\text { edge_property\& } & \text { ep, } & \text { bool } \\
\text { multi=true) } & & \end{array}$ & Add an edge. \\
\hline $\begin{array}{l}\text { void add_edge_async(const } \\
\text { edge_descriptor\& ed, bool } \\
\text { multi=true) }\end{array}$ & Add an edge asynchronous. \\
\hline $\begin{array}{l}\text { void add_edge_async(const } \\
\text { edge_descriptor\& ed, const edge } \\
\text { property\& ep, bool multi=true) }\end{array}$ & Add an edge with given property asynchronously. \\
\hline $\begin{array}{l}\text { bool delete_edge(const } \\
\text { edge_descriptor\& ed) }\end{array}$ & Delete the edge identified by its descriptor. \\
\hline $\begin{array}{l}\text { size_t } \\
\text { const }\end{array}$ get_num_vertices(void) & Returns the total number of vertices. \\
\hline $\begin{array}{l}\text { size_t get_local_num_edges(void) } \\
\text { const }\end{array}$ & Returns the number of edges on the local storage. \\
\hline size_t get_num_edges(void) const & Returns the total number of edges. \\
\hline void clear(void) & $\begin{array}{l}\text { Erase all vertices and edges in the graph; reset dis- } \\
\text { tribution/partition. }\end{array}$ \\
\hline $\begin{array}{l}\text { vertex_iterator } \\
\text { find_vertex(gid_type gid) }\end{array}$ & $\begin{array}{l}\text { Returns a vertex iterator corresponding to the GID } \\
\text { argument. }\end{array}$ \\
\hline $\begin{array}{lr}\text { bool } & \text { find_edge(const } \\
\text { edge_descriptor\& } & \text { ed, } \\
\text { vertex_iterator\& } & \text { vi, } \\
\text { adj_edge_iterator\& ei) } & \end{array}$ & $\begin{array}{l}\text { Returns a vertex iterator corresponding to the } \\
\text { source vertex of the edge ed and an edge iterator } \\
\text { of the edge. }\end{array}$ \\
\hline
\end{tabular}

Partitions that can be used with generic relational pContainers are arbitrary 
maps where individual GIDs can be map randomly to sub-domains. Block and block cyclic partitions can be used for regular pGraphs with fixed number of vertices.

\section{Sequence pContainer}

For conformance with the STL taxonomy, we provide the Sequence as a relation container with an implicit relationship (next). The pList implements the sequence interface while the pVector implements both sequence and indexed. The sequence interface is included in Table XVIII.

Table XVIII.: Sequence pContainer interface.

\begin{tabular}{|c|c|}
\hline Template Arguments & Description \\
\hline Traits & pContainer traits; \\
\hline Method & Description \\
\hline $\begin{array}{l}\text { void } \quad \text { push_back(const } \\
\text { value_type\& val) }\end{array}$ & Add a new element at the end of the container. \\
\hline void pop_back() & Remove an element at the end of the container. \\
\hline $\begin{array}{l}\text { void } \quad \text { push_front(const } \\
\text { value_type\& val) }\end{array}$ & $\begin{array}{l}\text { Add a new element at the beginning of the con- } \\
\text { tainer }\end{array}$ \\
\hline void pop_front() & Remove an element at the end of the container. \\
\hline $\begin{array}{l}\text { void insert_element_async(const } \\
\text { gid_type\& gid, const } \\
\text { value_type\& val) }\end{array}$ & $\begin{array}{l}\text { Insert asynchronously a new element before gid } \\
\text { specified. }\end{array}$ \\
\hline $\begin{array}{l}\text { gid_type insert_element(const } \\
\text { gid_type\& gid, const } \\
\text { value_type\& val) }\end{array}$ & $\begin{array}{l}\text { Insert synchronously a new element before gid } \\
\text { specified. Returns the GID of the newly inserted } \\
\text { element. }\end{array}$ \\
\hline $\begin{array}{l}\text { void } \quad \text { erase_element(const } \\
\text { gid_type\& gid) }\end{array}$ & $\begin{array}{l}\text { Erase the element corresponding to the specified } \\
\text { gid. }\end{array}$ \\
\hline $\begin{array}{l}\text { void } \\
\text { push_anywhere_async(const } \\
\text { value_type\& val) }\end{array}$ & $\begin{array}{l}\text { Add an element to the pContainerat an arbitrary } \\
\text { position. }\end{array}$ \\
\hline reference get_anywhere() & $\begin{array}{l}\text { Returns a reference to a random element in the } \\
\text { pContainer. }\end{array}$ \\
\hline void remove_element() & Remove a random selected element \\
\hline
\end{tabular}




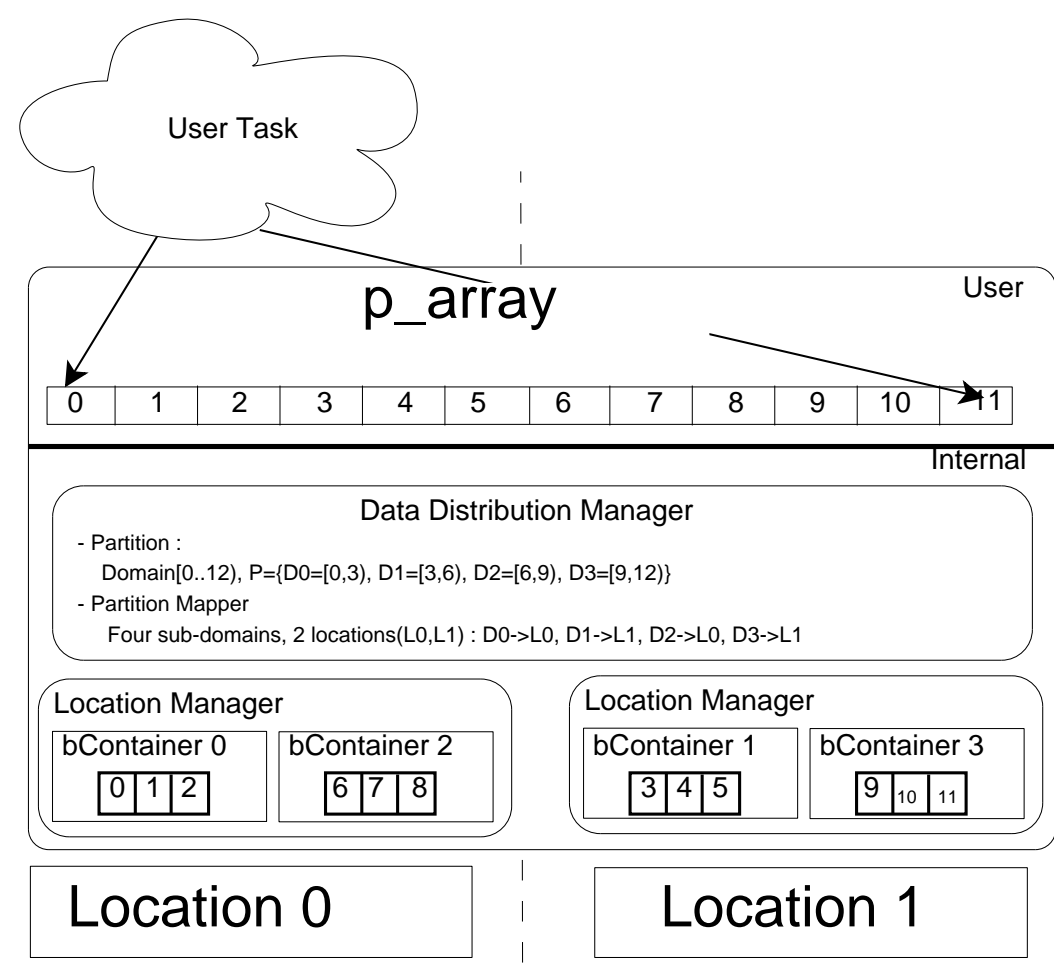

Fig. 10. Example of pContainer deployment on two locations.

E. Integrating all Concepts using pArray Example

In this section we exemplify how all concepts described in Section B are integrated together to implement a simple pArray data structure. The STL valarray container is a fixed size data structure optimized for storing and accessing data based on one dimensional indices. The STAPL pArray is the parallel equivalent of the STL valarray, providing an efficient interface to access data elements using indices. More information about the complete interface is included in Chapter IX.

Global Identifiers (GIDs) and Domains: For the pArray, the GIDs are the indices of the elements. The pArray domain is the universe of GIDsthat identify its elements and is represented as an integer range corresponding to the indices of the 
elements (e.g., 1DRange(0,12) ). The pArray's domain is a total ordered domain that specifies how elements are traversed by iterators of the default view. The pArray constructors accept an unsigned integer as an argument $(N)$ that internally is converted into a finite order domain $(1 \mathrm{DR}$ ange $(0, n))$ and the resulting index space and order of the elements will be as specified by the domain. It is trivial to extend the interface to have the pArray with an arbitrary domain, e.g., p_array $<>($ Domain $(5,12))$. This will declare a pArray whose first and last elements have indices 5 and 11, respectively. In Figure 10, the pArray has the range $[0,12)$ as its domain.

Partition The pArray is a static container, e.g., we can use blocked partitions using a block_size as an argument. Assuming $N$ is the size of the domain to be parti-

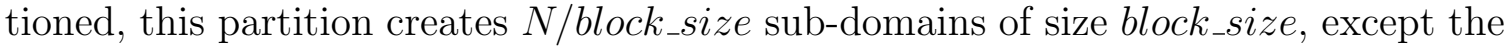
last one which may be smaller. Other partitions for pArray are balanced partitions, that will divide the elements of the domain into the specified number of sub-domains, each of whose size is $N / \#$ sub_domains. Explicit partitions are built by explicitly enumerating the sub-domains. The STAPL pArray can be built with any of these partitions. An important feature of STAPL is that the well-defined partition interface enables advanced users to implement their own partitions.

In Figure 10 we show the pArray with a blocked partition with blocks of size 3 . The corresponding sub-domains that this partition strategy (split) generates for the input domain $O D=([0,12), \leq)$ are:

$P=\left\{O D_{0}=[0,3), O D_{1}=[3,6), O D_{2}=[6,9), O D_{3}=[9,12)\right\}$

Partition Mapper: A partition is mapped onto a set of locations using a partition-mapper, which maps a sub-domain identifiers to a location. Any of the mappers introduced in Section B.5 can be used with the pArray data structure. Additional mappers with more information about the machine and interconnect can be implemented by users provided the interface included in Table IX is implemented. In 
Figure 10 we show the blocked partition $P=\left\{O D_{0}, O D_{1}, O D_{2}, O D_{3}\right\}$ being mapped onto available locations in a cyclic fashion. Thus sub-domain $O D_{0}$ is mapped to location $0, O D_{1}$ is mapped to location $1, O D_{2}$ is mapped to location 0 and $O D_{3}$ is mapped to location 1.

Storage bContainer: The pArray associates with every sub-domain of the partition a bContainer for data storage. The bContainers are implemented as STL valarrays. In Figure 10 we show the pContainer with two location managers instances, one in each location were the pContainer's data will reside. Each location manager handles the bContainers for the sub-domains that were determined by the partition and partition mapper.

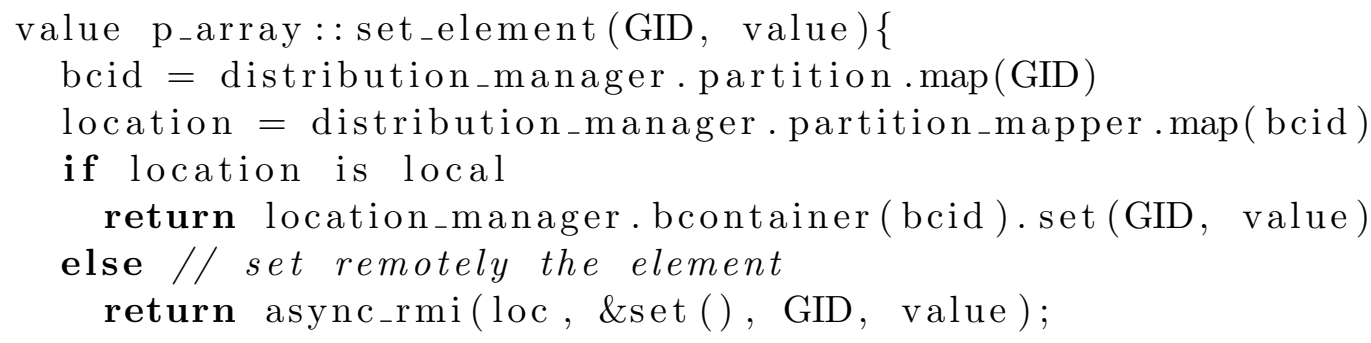

Fig. 11. Pseudocode of pArray set() method.

The simplified code for the pArray method set_element is shown in Figure 11 to illustrate how the pArray modules interact. The complete method performs additional actions as described in this chapter, Section B.6 and Chapter VI, Section B. The runtime cost of the methods in the pArray interface has three main constituents: the time to decide the location and the bContainer in which the element 


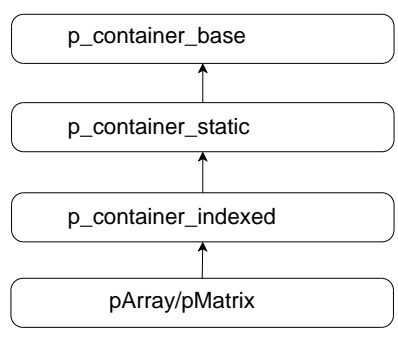

(a)

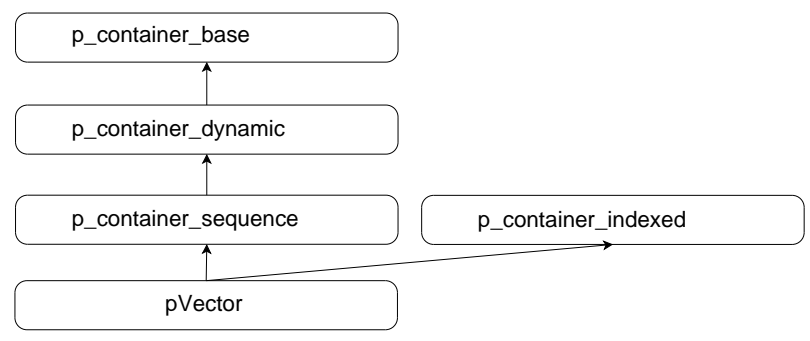

(d)

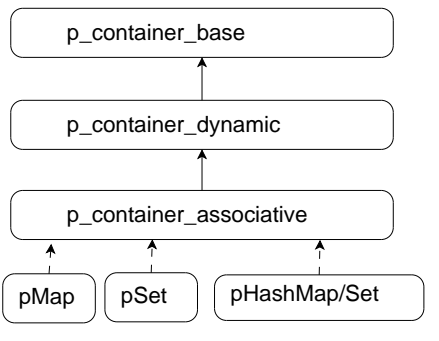

(b)

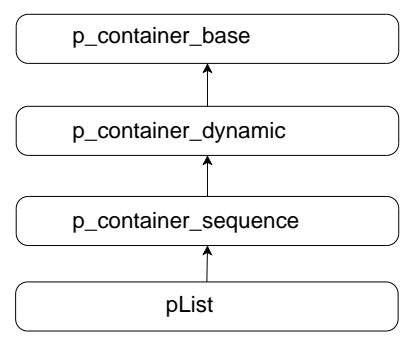

(c)

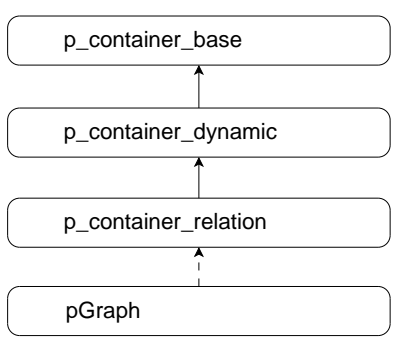

(e)

Fig. 12. pContainers inheritance. The most derived classes inherit all the methods of the base classes. A dotted line denotes that other classes are inheritted as explained in Chapters XI and XII.

is stored (Figure 11, lines 2-3), the communication time to get/send the required information (Figure 11, line 7), and the time it takes to perform the operation within a bContainer, which is currently an STL valarray (Figure 11, line 5).

F. pContainers Implemented in the Framework

STAPL provides a collection of commonly used pContainers that are constructed using the PCF. This includes counterparts of sTL containers (e.g., pArray [63], pVector, pList [65], and associative containers such as pSet, pMap, pHashMap, pMultiSet, pMultiMap [64] and additional containers such as pMatrix [15], and pGraph. In Figure 12 we depict the relationship between these pContainers and the classes of the 
framework.

The pArray and pMatrix (Figure 12(a)) are indexed containers using one or two dimensional indices (GIDs) respectively. The interface for these simple data structures is mainly provided by the base classes with the final classes providing only constructors and necessary type definitions. The complete interface for the pArray is discussed in Chapter IX. The pMatrix is described in more detail in [15], and provides a similar interface with the pArray. The pList (Figure 12(c)) derives from the sequence pContainer from which it inherits a reach interface to add and erase elements at the begining, the end and an intermediate point in the sequence. The complete interface for pList, discussed in more detail in Chapter X implements all the methods in the sequence interface in constant time. The pVector is a sequence pContainer that also implements the indexed pcontainer interface as shown in Figure $12(\mathrm{~d})$. Due to constraints on complexity for the indexed interface the pVector incurs a bigger overhead when implementing insert operations. While both pList and pVector are two sequence pContainers providing similar interfaces, there is a well known performance/usability tradeoff between the two. The pVector provides constant access time to the elements based on their indices, linear time for inserts, and amortized constant time for push back type methods. The pList does not provide random access to the data based on indices but implements dynamic operations such as insert and push back in constant time. Depending on the particular needs of an application, these two data structures can be used for different computational phases with possible conversions from one to the other. More details about the tradeoffs between pList and pVector are included in [65].

Associative pContainers provide efficient storage for elements based on keys. They include sorted associative containers, which guarantee logarithmic access time to the elements, and hashed associative containers that guarantee amortized constant 
time. They provide a simple interface that includes insert find and erase as described in more detail in Chapter XII. The pGraph is a relational pContainer consisting of a collection of vertices and relations between vertices called edges. The framework provides a relational pContainer base that implements a minimal interface to add and delete vertices and edges. Additional functionality is supported by the pGraph specific classes as described in Chapter XI. 


\section{G. pContainer Support for Redistribution}

One of the design goals of the PCF is to allow pContainers with various partitions and mapping on the machine. This can be achieved by specifying the desired partitions and partition mapper as template arguments at compile time. The data redistribution is the process of reorganizing the data of a pContainer based on a new data distribution (new partition and/or partition mapping) as described in Chapter IV, Section C. We integrate into the PCF support for allowing an individual pContainer to change its partition and partition mapping dynamically during the execution. This is achieved by using polymorphic implementations for both the partition and partition mapper and providing the necessary support to move data across different locations. For example, for pArray we mentioned we currently support balanced, blocked and explicitly blocked partitions. These three types of partitions can be interchanged within the same pContainer instance dynamically. In this section we describe the support implemented in the PCF to allow this functionality.

The Partition Proxy is a polymorphic wrapper for real partitions and provides the necessary support to change the underlying partition at runtime. There are some trade-offs when using a partition proxy. While giving users more flexibility it involves virtual methods with the associated overhead and missed opportunities for compile time optimizations. The default partitions of all pContainers are proxy partitions and the framework provides proxies for all components of the taxonomy: partition_proxy_indexed, partition_proxy_dynamic, partition_proxy_sequence, partition_proxy_associative, and partition_proxy_relation. When a partition proxy is used, the interface of the pContainer is automatically extended with the the following interface:

1 void redistribute (new_partition [, partition_mapper ]);

2 void redistribute (redistribution_map); 


\begin{tabular}{|c|c|c|c|}
\hline Partition 1 & SD_0 & $\mathrm{S}$ & SD_1 \\
\hline Partition 2 & SD_0 & $\mathrm{s}$ & $\overline{\mathrm{SD} 1}$ \\
\hline
\end{tabular}

Fig. 13. Redistribution for two given partitions. Section $S$ (one or more elements) of sub-domain 1 in first partition will migrate to sub-domain 0 in the second partition.

Trying to invoke the above methods on a pContainer with a non proxy partition will generate a compiler error.

While performing the redistribution, there is the new partition and/or partition mapper and the original ones. A naive redistribution approach can simply create a new pContainer organized according to the new partition, copy data from the old storage and delete the old storage. However this is a very inefficient approach. To assist users in performing efficient redistribution, we introduce the redistribution map which contains only the elements that will migrate from a sub-domain to a new sub-domain. A common case occurs when the repartition moves elements across neighboring sub-domains. The redistribution map will benefit the redistribution in this situation. In Figure 13, we depict a case were the redistribution will have to migrate only the data corresponding to sub-domain $S$ in order to match the second partition. The framework will provide a set of predefined constructs for common redistribution maps while the users will provide their own for more specific patterns.

Some simple redistribution patterns that can be easily provided by the framework are:

- rebalance() : Redistribute the $N$ elements of the pContainer across $P$ locations such that each location will own $N / P$ elements

- rotate (how many positions, direction) : Redistributes the elements of the 
pContainer by cyclically rotating them a given number of locations in a given direction.

- custom redistribution for certain pContainers: transpose for two dimensional containers, graph redistributions, etc.

\section{Data Marshaling}

To support the default redistribution, the framework requires that both pContainer data elements and bContainers be marshaled. Support for data marshaling is provided by the STAPL RTS[54] and requires users to implement a define_type() method as part of the class that needs to be marshaled. The bContainer interface includes the define_type as part of its required interface. This can easily be achieved as STAPL provides built in support for all STL containers and these are the building blocks for most bContainers we employ in our pContainers.

In Figure 14 we include examples showing the define_type() implementation for some simple classes and for the pArray bContainer. The method receives as input an object of type stapl: : typer. Subsequently, the typer is made aware of all the class data members and this process continues in a recursive fashion. In Figure 14(a), class classB has as a data member an object of classA so the define type of classB will recursively invoke the define type of it. In Figure 14(b), we show the define_type for the pArray bContainer which invokes recursively the define type of the STL valarray data member.

With bContainer marshaling support available, the pContainer redistribution implementation is greatly simplified. The redistribution map specifies the sub-domains and their new locations. Data corresponding to sub-domains is appropriately packed in bContainers and shipped to the destination 


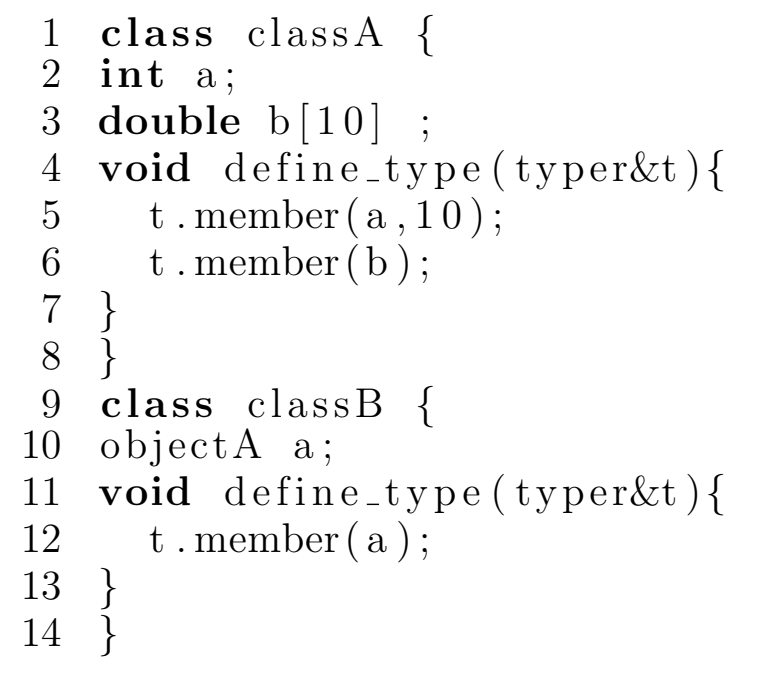

(a) Simple classes

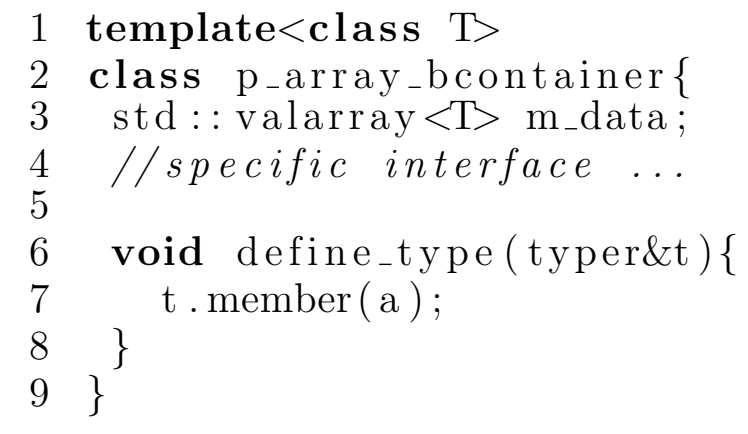

(b) pArray bContainer

Fig. 14. Marshaling interfaces.

\section{H. pContainer Customization using Traits}

When building a pContainer, a developer has the ability to customize the pContainer main functional modules as described in Chapter IV, Section B. For example, a developer may want to use a certain storage or certain partition and mapping on the machine, enforce a certain memory consistency model, or enable/disable thread safety, etc. This leads us to design the PCF in a very configurable way where users can select individual functional modules. This is accomplished using traits classes that are passed as template arguments to pContainer base classes. The traits can be customized by developers and end users for classes of pContainers or even on a per pContainer instance.

In addition to the data structure specific arguments, all pContainer classes take as template arguments the partition and the pContainer traits. For example, in 


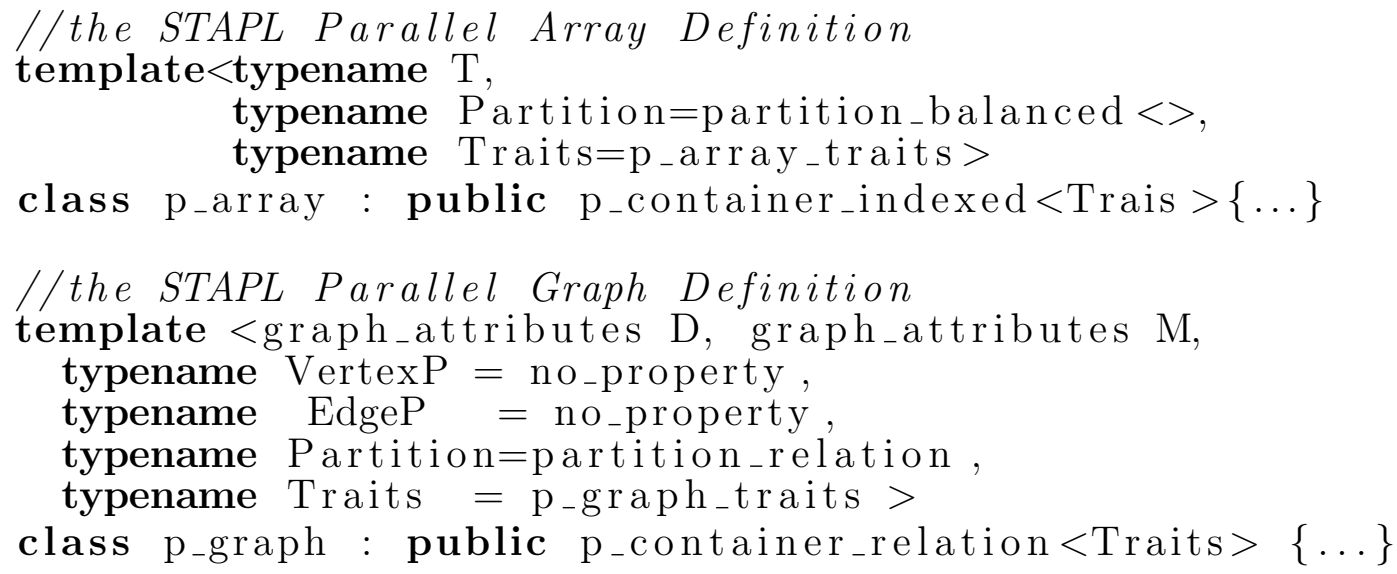

Fig. 15. pContainer template arguments.

Figure 15 we list the definition of the pArray and pGraph; more detail on these specific data structures is provided in Chapter IX and XI, respectively:

The partition specifies the GID, domain, bContainer, and thread_safety_manager. The pContainer will use the definition for these types from the partition. The pContainer traits can be used to specify lower level details such as partition_mapper, data-distribution-manager and location-manager. We expect these to be less often customized by users than the storage and partition.

When interacting with the framework users can provide alternative implementations for domain, partition, bContainer, partition mapper and location-manager using the template arguments. The custom provided modules need to implement the required interfaces as specified in Sections B and D.

In Figure 16 we show an example of STAPL pseudocode illustrating how users can customize an existing pGraph implementation. Users can select the storage by providing the type of an existing bContainer and similarly for the partition. Figure 16, line 5, shows the declaration of a directed pGraph allowing multiple edges between 


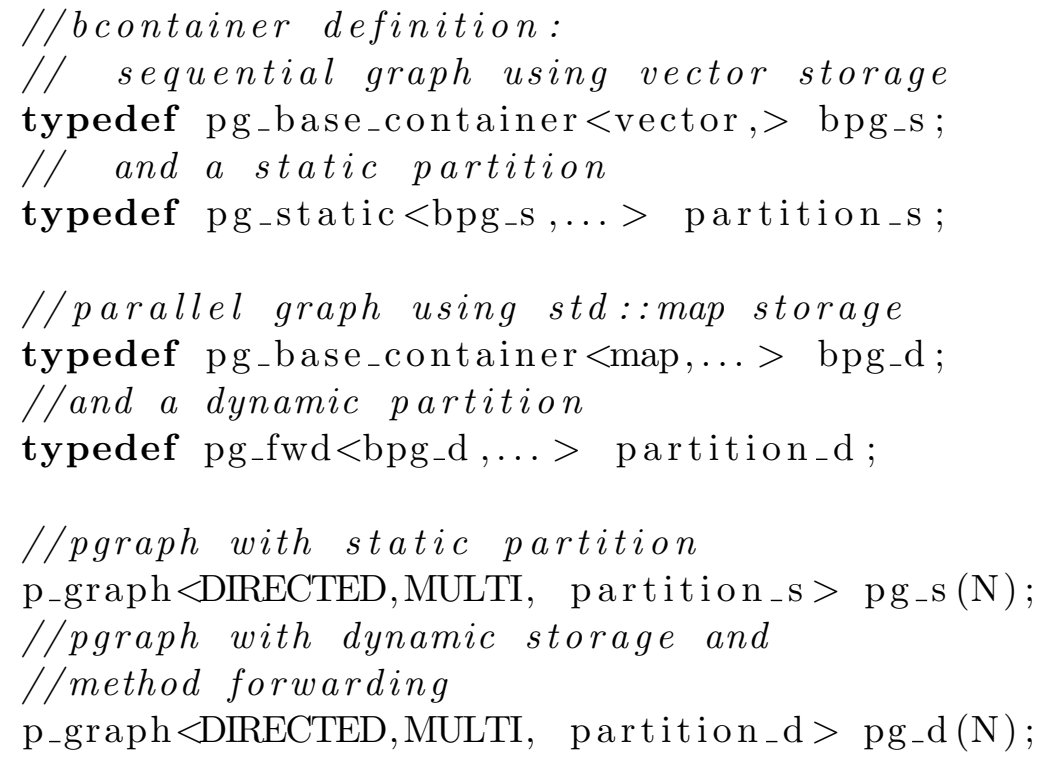

Fig. 16. pGraph customization.

the same source and a target vertex and using a static partition. With a static partition, users need to declare the size of the pGraph at the construction time and subsequent invocations of the add_vertex method will trigger an assertion. Figure 16, line 6, shows the declaration of a pGraph using a dynamic partition that allows for addition and deletion of both vertices and edges. More details and performance results regarding the benefits of having different partitions and types of storage are discussed in Chapter XI, Section F.2 in the context of a dynamic pGraph data structure. 


\section{CHAPTER VI}

\section{THREAD SAFETY}

In this chapter, we describe the infrastructure provided by the pContainer framework to implement thread safe pContainers. The goal of STAPL is to allow users to easily develop thread safe containers while giving them the possibility to override the default locking policies implemented by the framework. We define that a pContainer is thread safe $[33,34,35]$ if concurrent method invocations from various threads are perceived as being atomic thus always leaving a pContainer in a consistent state.

We start by first motivating the need for thread safety. Data stored in pContainers is accessed by pAlgorithms and at this level STAPL employs the task dependency graph (TDG) to encode data dependences across various tasks of a computation. However there are a large number of parallel algorithms employing commutative tasks. In this case, two tasks $\mathrm{A}$ and $\mathrm{B}$ are safe to be executed in $A \rightarrow B$ order (A followed by B) or $B \rightarrow A$ but not $A \| B$ (A in parallel with B). For such scenarios the dependencies between two tasks can be eliminated under certain conditions as long as atomicity of the pView and pContainer method invocations are guaranteed.

As a simple example consider the sample sort parallel algorithm where each task inserts elements from an input pArray into a pArray of pVectors (buckets). This computation can be expressed as a no dependency TDG where each task determines the bucket an element belongs to and inserts the element into that bucket. This algorithm will properly insert all the elements into buckets as long as atomicity at the bucket level is guaranteed. 


\section{A. pContainer Thread Safety Design}

As introduced in Chapter V, Section A, a pContainer is implemented as a collection of existing data structures to store data (e.g., bContainers) augmented with information for parallelism management or meta data (e.g., partition, partition mapper, thread safety manager).

When designing the pContainer thread safety mechanisms we considered the following:

- A pContainer method in general accesses and/or modifies both metadata and data so these two entities need to be considered in an integrated approach.

- A pContainer method invocation typically involves more than one location due to the forwarding mechanism introduced in Chapter V, Section C. This means that a pContainer method may access only metadata on certain locations while on other locations it may access both data and metadata.

- For most pContainers, the bContainer implementation is a black box. This is encapsulation and it is key to object oriented programming. When performing operations using the public interface we do not know what memory addresses are touched inside the bContainer, especially by dynamic operations such as insert/erase. For example, while an insert in a vector may touch all memory addresses for all elements encompassed in it, a user is not generally aware of it. For this reason, the framework takes a high level general approach where thread safety can be provided with or without support at the bContainer level.

- The framework has to be able to integrate existing thread safe and non-thread safe data structures to store both data and metadata. This is a crucial requirement in order to incorporate a large body of work that has been done in the 
area of thread safe data structures for shared memory machines and multicores [20, 24, 26, 33, 34, 37].

- The framework needs to provide a generic, customizable solution, where different custom locking policies can be used with a particular pContainer. A desired thread safety manager can be selected by the user using custom traits.

Based on the above requirements we implemented the pContainer thread safety as a set of specifications across the following PCF modules:

- Distribution Manager: implements pContainer methods providing call back points to classes responsible for thread safety. This allows for customizable locking policies. See Section B.

- Thread Safety Manager: provides a generic interface such that users can control the type of mutual exclusion mechanism desired. See Section C.

- Partition: specification for the locking modes and the locking granularity performed by the thread safety manager.

- bContainer: locking at the base container level.

\section{B. Data Distribution Manager}

This module ensures the safe access to data and metadata by employing a thread safety manager. The data distribution implements a generic method skeleton such that individual pContainer element-wise methods are implemented as invocations of this generic method with customized functors to determine the location where the method will be executed and how it will be effectively executed. This was described in more detail in Chapter V, Sections C.4 and C.6. In Figure 17, we include the generic 
method invoke presented in Chapter IV, Section B.6 but this time augmented with the locking support.

As depicted in Figure 17, the pContainer framework informs the thread safety manager about the actions it is about to perform. All the decisions on the granularity and type of locking to be performed are completely managed by the thread safety manager. This is either a default implementation or is provided by the user. It uses knowledge about the particular metadata and data implementation used to perform adequate locking. Please note that the framework doesn't implement a particular locking algorithm (there is no best one) but it enables users/developers to perform custom locking according to their specific data structure.

\section{Thread Safety Manager}

A thread safety manager class will be associated with every pContainer. The thread safety manager maintains the necessary information to ensure atomic updates to data and metadata of the pContainer. The functionality of this module will be employed by the PCF when thread safe access is required. The interface of the thread safety manager is described next:

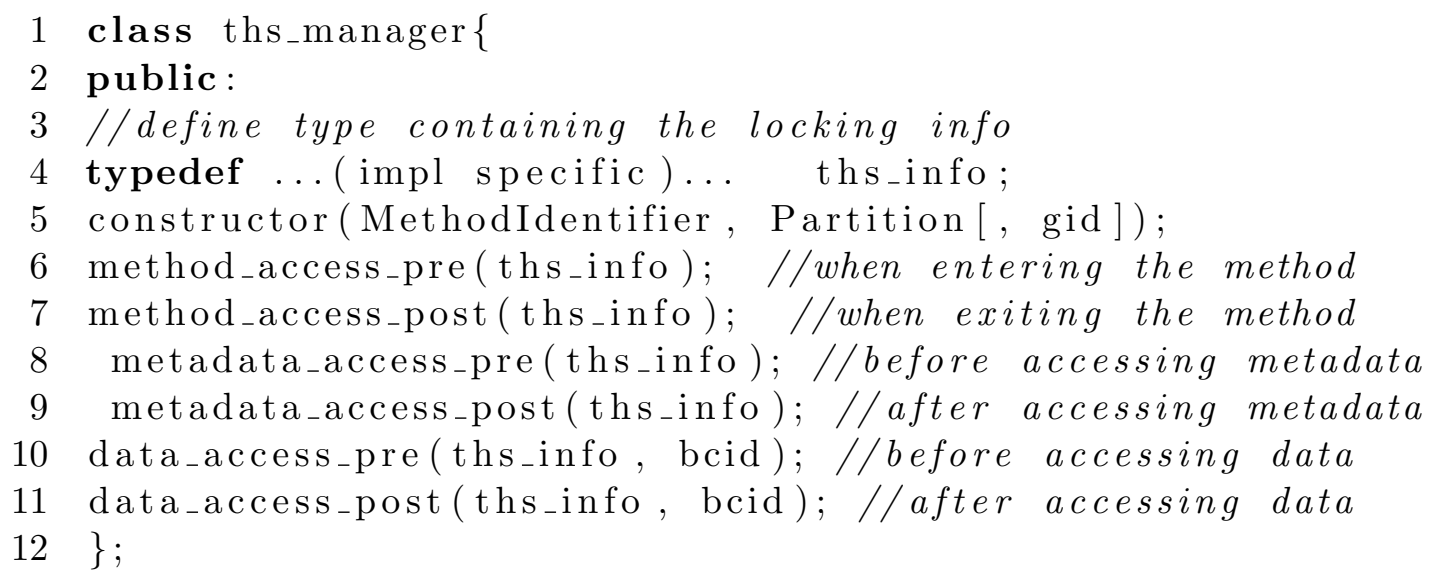




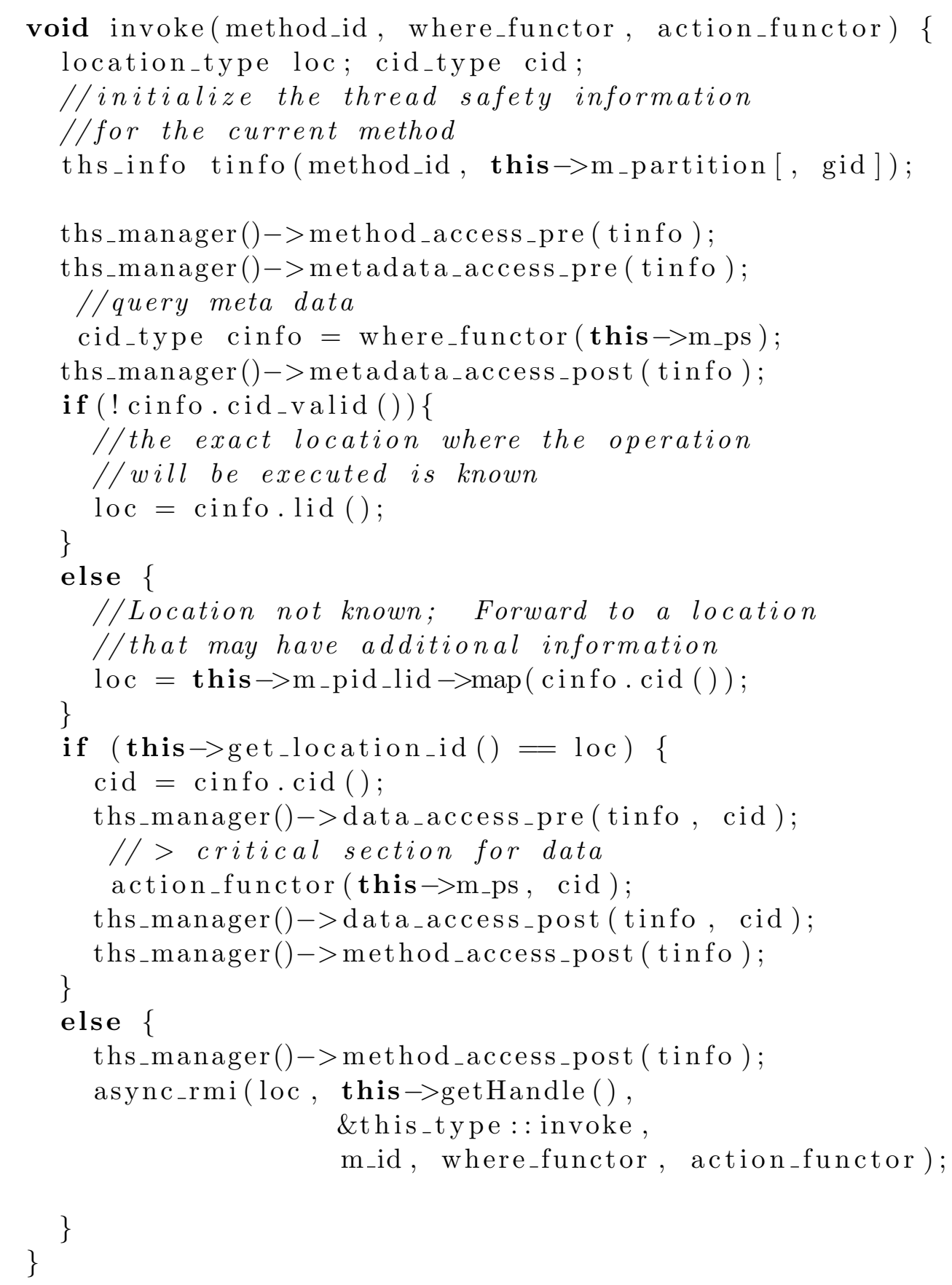

Fig. 17. Generic invoke method implementation with locking statements. 
In the specification above, the MethodIdentifier is an integer value uniquely associated with a pContainer method. The partition will define appropriate locking attributes for each of the pContainer methods as described in the next section.

\section{Partition Locking Specification}

A STAPL pContainer is designed to support different partitions with different locking modes for the methods in its interface. This is accomplished by routing all pContainer methods to the distribution manager, which in turn forwards to the partition class. Users can customize this behavior by specifying partition classes. This way they can specialize existing partitions or implement new ones with custom locking.

The partition class allows pContainer users to specify attributes for each individual method regarding the granularity of data accesses and the type of access. For example different methods may access an element in a bContainer (e.g., get_element()), the entire bContainer (e.g., insert into a vector) or all bContainers (e.g., size()). Corresponding to these three situations, the framework specifies the following identifiers: ELEMENT, BCONTAINER, LOCAL. Additionally each individual method accesses the data and metadata in a READ or WRITE mode, information that can be used in conjunction with read/write locks to allow multiple readers shared access to the data.

A special tag that can be used when specifying the granularity is NONE, which is used to inform the thread safety manager than no action is required. This can be the case for example for a read only static data structure such as pArray or pMatrix. Advanced uses of different thread safety attributes are envisioned where users can modify them dynamically for different computation phases. Below we include the 
specification of the method attributes used by default by the pVector.

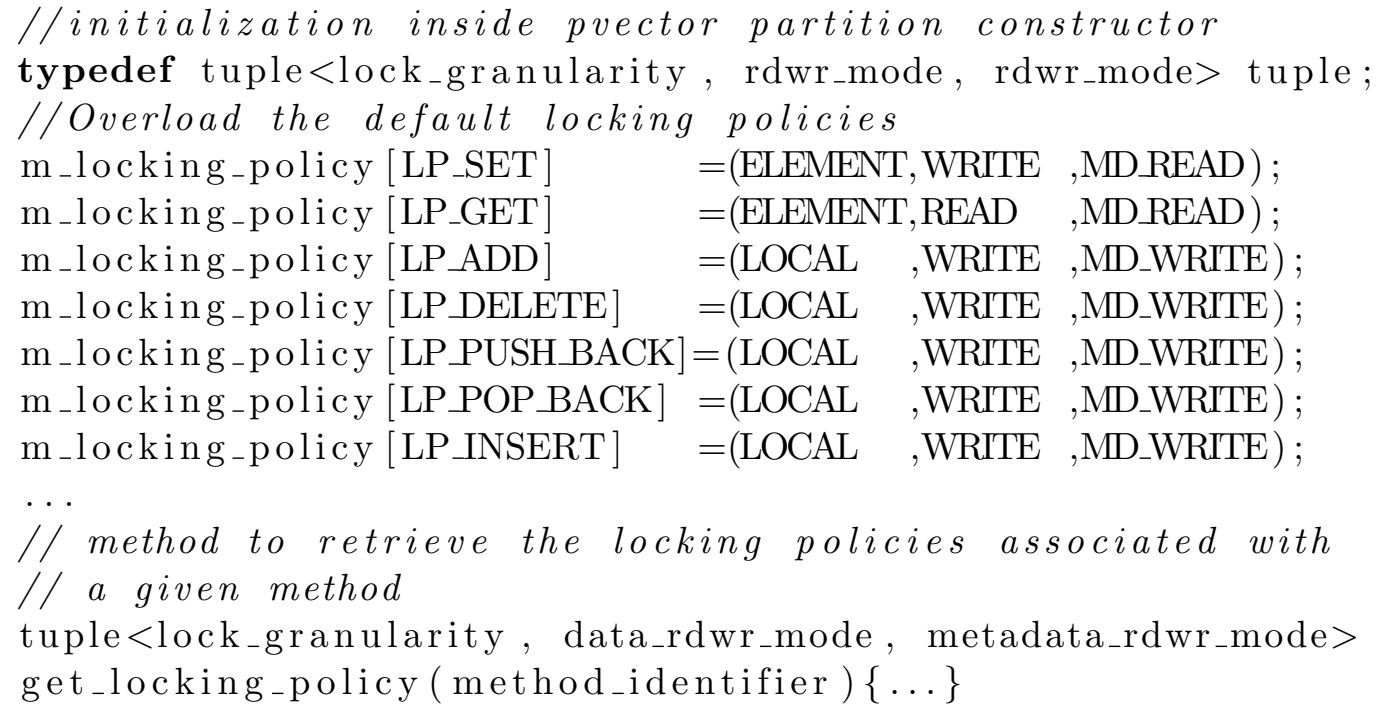

A thread safety manager uses the above specification to perform appropriate actions (e.g., locking) when correspondingly invoked by the pContainer methods. In the remainder of this chapter we discuss various thread safety managers that can be used with STAPL pContainers.

E. pArray and pMatrix

Default implementation: The meta data is static and read only for all elementwise methods. Only data/bContainers are locked when accessing elements for read or write.

Customizations: Using traits, a custom thread safety manager can be specified that performs no locks. This may be useful for read only pContainers or for the case when concurrent accesses are taken care of by the task dependency graph of the application.

An interesting optimization that can be used to refine the lock granularity is to 
provide a thread safety manager that uses $K$ locks where $K$ is arbitrary and each individual data access for a GID is hashed to one of the locks and that lock will be used when accessing the data for the given GID.

F. pList

Default implementation: The meta data is read only for all element-wise methods. Only data/bContainers are locked when accessing elements for read or write. Meta data is modified by collective operations and guarded by fence.

Customizations: Using traits, a custom thread safety manager can be specified that performs no locks. This may be useful for read-only pContainers or for the case when concurrent accesses are taken care of by the task dependency graph of the application.

Thread safe bContainers can be used, in which case no locking is performed by the framework.

G. Associative pContainers

Default implementation: The meta data is either static or closed form. Only data/bContainers are locked when methods are executed.

Customizations: Using traits, a custom thread safety manager can be specified that performs no locks. This is useful for the case when concurrent accesses are taken care of at the prange level.

Thread safe bContainers can be used, in which case no locking is performed by the framework. 
H. pGraph

Default implementation: The meta data for a pGraph is maintained in a parallel map. It is dynamic and modified by every add/delete vertex. In general the vertices are added on one location and meta data inserted on another location. So adding a vertex is naturally broken into two individual operations. Since the meta data holder is thread safe, the pGraph performs no locking for it. Only when accessing the data in a bContainer locking is invoked.

Customizations: A static partition where the number of vertices is fixed at construction time. This kind of graph will allow for edges to be added/deleted. The atomicity can be enforced using a set of locks (or a set of locks per bContainer) and a hashing scheme to decide the lock to use based on the GID.

A thread safe graph bContainer can be employed to eliminate the locking performed by the framework. 


\section{CHAPTER VII}

\section{MEMORY CONSISTENCY MODEL}

In this section we describe the memory consistency model (MCM) provided by STAPL pContainers. This consists of a set of guarantees about the termination, ordering and values returned by the pContainer method invocations that users rely upon when reasoning about the correctness of their applications $[6,46,1,3,2]$. The pContainer provides a shared memory view to the user while storing its data distributed on different memory address spaces. In Figure 18 we depict a pContainer that stores a pContainer representative on all locations where it will distribute its data. Each representative stores a subset of the data and there is no data replication across locations. The pContainer methods hide the distributed nature of a pContainer and allow users to access the data elements in a shared memory fashion. Users interact with a pContainer through a series of method invocations and corresponding responses. The memory consistency model is the set of guarantees provided to the users about the possible values returned by methods or when responses or acknowledgments that an operation is finished are received.

For the STAPL PCF, we considered a modular design where different memory consistency models can be employed by different pContainers. Similar to other pContainer properties this can be customized using the traits template arguments. In Section A we show general considerations for all pContainer methods and in Sections $\mathrm{B}$ and $\mathrm{C}$ we introduce the default memory consistency model provided by STAPL pContainers. In Section E we show how the default memory consistency model can be constrained or relaxed to implement other models. 


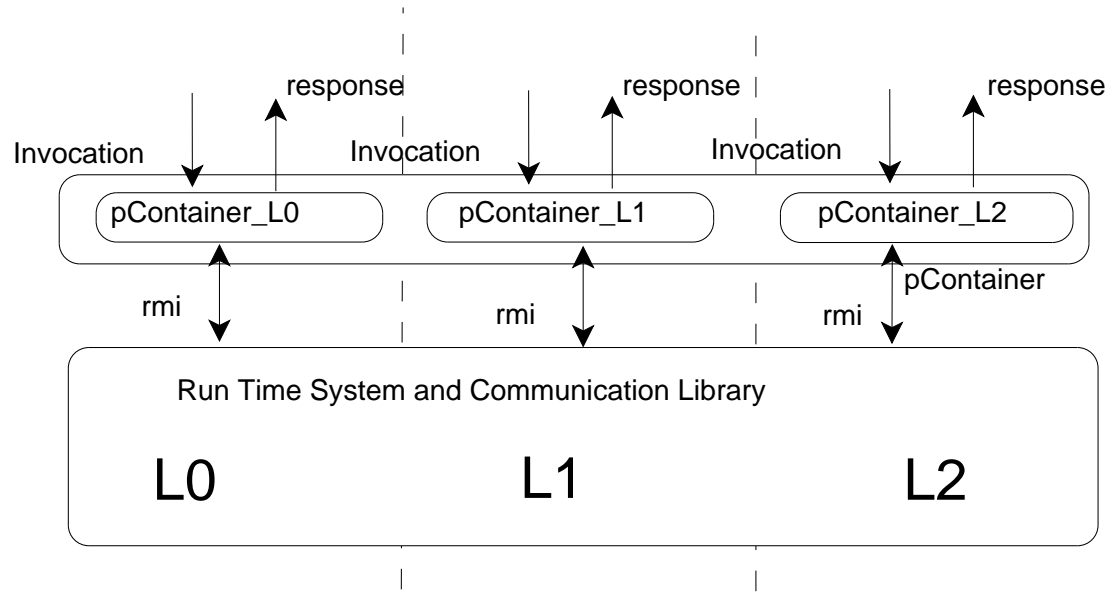

Fig. 18. User, pContainer, run time system interaction.

\section{A. pContainer Interfaces}

As described in Chapter V, Section B the pContainer interface contains the following categories of methods: collective (COLL), synchronous (SM), asynchronous (AM) and split phase (SPM). Each of these types of methods provides different guarantees about the completion of the invocations and ordering among them when invoked concurrently from multiple threads. The complete specification of the guarantees provided to the user is the pContainer memory consistency model (MCM).

To further motivate the need for a MCM for the pContainers we include in Figure 19 an example of a simple STAPL program to exemplify how users can rely on the MCM guarantees to reason about the correctness of their application. The simple program starts by instantiating a pArray container. This is a collective operation that will build a pArray representative on all locations. The acknowledgment that the constructor is finished is received when the constructor returns. Next we build a pView on the pArray's data. Similarly, there is a pView representative on all 
locations and the acknowledgment the pView is built is received when the pView constructor returns. Next, we spawn a number of parallel tasks using the map_func STAPL construct. The map_func takes as input a pView representing the data and a work function that is applied to data in the pView. For the map_func included in Figure 19 we assume the STAPL infrastructure creates a task for each location where the pView has a representative. The tasks will be scheduled and run in parallel by the STAPL RTS. The code that each task executes does not have to be the same across all tasks.
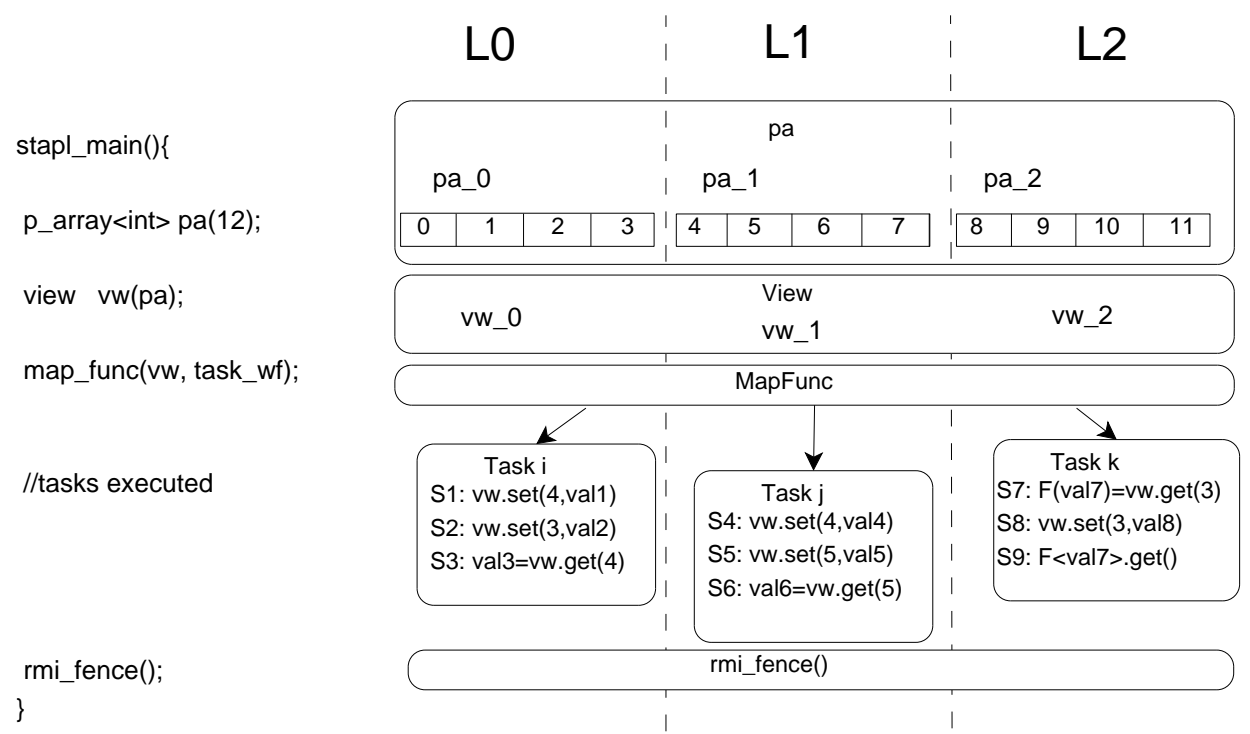

Fig. 19. STAPL program execution.

For the example in Figure 19 we assume all initial elements in the pArray are zero. When Task $_{i}$ executes $S 1$ we notice that this can be executed concurrently with $S 4$ from Task $_{j}$ and the question is what value the read of pa [4] in $S 3$ may 
return. The MCM describes the completion and ordering guarantees of operations that are concurrently executed by multiple threads of computation as we describe in the following sections. We come back to the example in Figure 19 in Section D.

\section{B. Completion Guarantees}

In Chapter III, Section B we introduced the RTS and described the sync_rmi, async_rmi and rmi_fence as the main communication and synchronization primitives. The rmi_fence construct is provided by the RTS to ensure that previously spawned messages are received on their target locations. There are currently two fence calls available in STAPL: rmi_fence collective call, where all threads in the execution group need to perform the invocation and one sided os_fence that can be invoked independently of the other threads of computation. From the individual thread's point of view, both fences ensure all acknowledgments for the pending operations are received. The global fence ensures the acknowledgments are globally received by all pending operations. The STAPL runtime provides additional primitives that subsume the fence semantic such as one-sided/collective reduce/broadcast.

We introduce in this section a terminology to specify the start and the termination of the pContainer methods and the specification of when pContainer methods are completed.

- Collective methods: start CollM and termination ACK_CollM (e.g., constructor). ACK_CollM is received by the invoking thread when the method returns.

- Synchronous methods: start SM and the termination is denoted as ACK_SM (e.g., get_element). For a $\operatorname{SM}(\mathrm{x})$, the $\mathrm{ACK}_{\mathrm{S}} \mathrm{SM}(\mathrm{val})$ is received by the invoking thread when the method returns. 
- Asynchronous methods: start AM and termination ACK_AM (e.g., set_element). For a $\mathrm{AM}(\mathrm{x})$ there is no explicit acknowledgment sent from the pContainer to the user. To reason about correctness we logically assume that the ACK_AM is received by the invoking thread at any point in the program order before any one of the following events:

- Encountering a fence call

- A subsequent $\operatorname{SM}(x)$ or $\operatorname{SPM}(x)$ receives its acknowledgment. Synchronous and split phase methods on an element $x$ of a pContainer forces acknowledgments for all pending asynchronous methods operating on the same element $x$.

- A subsequent $\operatorname{AM}(\mathrm{x})$ receives its acknowledgment. Asynchronous method invocations from the same thread and on the same element $x$ of a pContainer receive their acknowledgments in order.

- Split phase methods: start SPM and termination ACK_SPM (e.g., split phase get element). The invocation of a split phase method returns immediately to the user a future. We denote the creation of the future as SFuture. The return value corresponding to the future is obtained by invoking the method get and we represent this as Future.get. The acknowledgment for the Future.get is denoted as ACK_F and it is received when the method returns. The acknowledgment of a split phase method and implicitly the value returned, is logically received by the invoking thread at any point in the program order after the future creation and before any one of the following events:

- Encountering a fence call.

- A subsequent $\operatorname{SM}(\mathrm{x}), \operatorname{AM}(\mathrm{x})$ or $\operatorname{SPM}(\mathrm{x})$ receives its acknowledgment. 
- When Future.get receives its acknowledgment ACK_F.

The fence receives its acknowledgment when it returns together with the acknowledgments of all pending asynchronous and split phase methods. Our framework guarantees that for all method invocations there is an acknowledgment and this is an important property of a distributed system referred to as liveness[6].

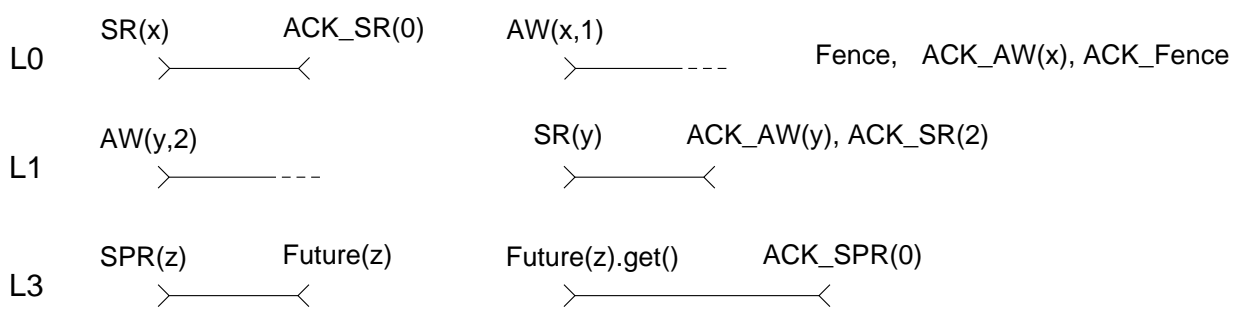

Fig. 20. Completion guarantees. The time increases from left to right.

In the following sections we use $\operatorname{SR}_{L_{i}}(x)$ to denote the beginning of a synchronous method that will read an element $x$ of a pContainer. We use $\mathrm{ACK}_{-} \mathrm{SR}_{L_{i}}($ val $)$ to denote the acknowledgment and the returned value. The index $L_{i}$ is a thread identifier and is used to distinguish among invocations in different threads. Similarly, for asynchronous write operations we use AW and ACK_AW and for split phase reads we use SPR and ACK_SPR. In Figure 20 we include a picture to exemplify the termination guarantees introduced in the previous section. We exemplify using asynchronous writes (AW), synchronous reads (SR), and split phase reads (SPR), but the same holds for any element-wise pContainer method. The example accesses elements corresponding to three different pContainer elements identified by their GIDs, $x, y, z$. For all examples 
considered in this chapter the elements have all value 0 initially. Each row in the figure contains method invocations in program order from left to right. Each invocation takes a certain amount of time which is represented as the length of the segment between start and acknowledgment. Location L0 performs a synchronous read of element $x$. The invocation returns ACK_SR(0) when the method returns. The next AW operation receives the acknowledgment before the subsequent fence returns. Location L1 performs an AW on $y$ followed by a SR on $y$. The SR operation since it is on $y$ implies the ACK_AW(y) and the ACK_SR(2). Location L2 performs a split phase read of $z$. The invocation returns immediately to the user a future. When the get method of the future is invoked the ACK_SPR(2) is received.

\section{Memory Consistency Conditions}

In sequential programming, invocations are completed in the order in which they were issued in the program. In a parallel system it is often the case that this requirement is relaxed in order to provide improved performance.

\section{1. pContainer Default Memory Consistency Model}

Based on the termination guarantees introduced in the previous section we now describe the default memory consistency conditions of the pContainer methods. We first introduce a set of notations and rules and later in this section we show the interaction of this rules with a set of examples.

We adapted from [6] a set of notations to formally specify the pContainer memory consistency model. Let $E$ be an execution of a program which has concurrent method invocations by multiple threads in multiple locations and on multiple pContainer elements. $E$ is a sequence of method invocations as they occurred as 
a result of interleavings of the actions of all the threads in the system. $E$ can be thought as a trace of a particular execution of a program. We use the notation $E \mid i$ to denote the subsequence of $E$ consisting of all invocations performed by and responses received by a thread $i$. Similarly we use $E \mid x$ to indicate the subsequence of $E$ consisting of all invocations and responses that are performed on an element $x$ of the pContainer.

To simplify reasoning about the possible method interleavings and values returned by an execution $E$, we introduce the notion of a permutation of the method invocations as a linear sequence of all method invocations in the system. The MCM specifies the restrictions on the possible permutations corresponding to a particular execution E. Fences serve as global synchronization points that force the completion of all previous pContainer methods. In the following we discuss the guarantees for the method invocations between fences.

The pContainer MCM: For an execution E, a pContainer guarantees that there is a permutation $P$ of all method invocations in $E$ such that:

1. The methods in $P$ occur sequentially (no overlapping).

2. For each element $x$, the restriction of $P$ to just those methods on $x$, denoted $P \mid x$, satisfies the specification of the data type of $x$. (E.g., if $x$ is a register that supports Read and Write, then each Read returns the value of the latest preceding Write invoked on $x$.)

3. For each thread $i$, the restriction of $E$ to just the Coll and Synch methods invoked by $i$, denoted $E \mid(C o l l \cup$ Synch $) \mid i$, must equal $P \mid($ Coll $\cup$ Synch $) \mid i$. That is, the permutation $P$ has all the collective and synchronous methods by $i$ in the same order as they were invoked. However, no guarantee is given as to how Synch methods at different locations are ordered in $P$. 
4. For each element $x$ and each thread $i$, the restriction of $P$ to the methods on $x$ invoked by $i$, denoted $P|x| i$, consists of all the Synch, Asynch, and Split Phase methods on $x$ invoked by $i$ in $E$, in the order of their invocation.

5. Consider any element $x$ and let $O_{i}$ and $O_{j}$ be two operations on $x$ in $E$ such that $O_{i}$ is invoked by some thread $i, O_{j}$ is invoked by some other thread $j$, and $O_{i}$ completes (i.e., receives its ACK) before $O_{j}$ is invoked. Then $O_{i}$ is ordered in $P$ before $O_{j}$.

In the remaining of this section we exemplify some of the ordering relations for pContainer methods as derived from the consistency conditions previously introduced.

For asynchronous methods the PCF guarantees that subsequent invocations from the same thread affecting the same element $x$ will receive their implicit acknowledgments in the order in which they were invoked (condition 4). For example, let us assume a thread in location $L 0$ performs the sequence of asynchronous write invocations depicted in Figure 21(a). The acknowledgment for two consecutive writes on $x\left(\mathrm{AW}^{0}(x), \mathrm{AW}^{1}(x)\right)$ are guaranteed to be in the order in which they were issued. The superscript is used to distinguish subsequent invocations of the same type on the same element/location. The acknowledgment for the write to $y$ has no relationship with the acknowledgment for $x$. Figure 21(a) shows three possible valid interleavings with the acknowledgment for $y$ received in an arbitrary order relative to the acknowledgments for writes to $x$. Corresponding to Figure 21(a) we include in Figure 21(b) three possible valid interleavings as perceived by the user.

When invoking methods concurrently on the same pContainer element from threads in different locations there is no guarantee about the order in which they will terminate. Let us assume the following method invocations from two different 
$L 0: \mathrm{AW}^{0}(x, 1), \operatorname{AW}(y, 1), \operatorname{AW}^{1}(x, 2)$ fence $\operatorname{ACK} \_A W^{0}(x), \operatorname{ACK}_{A} \mathrm{AW}^{1}(x), \operatorname{ACK} \_\mathrm{AW}(y)$

or

$L 0: \operatorname{AW}^{0}(x, 1), \operatorname{AW}(y, 1), \operatorname{AW}^{1}(x, 2)$ fence $\mathrm{ACK} \_\mathrm{AW}^{0}(x), \operatorname{ACK} \_\mathrm{AW}(y), \mathrm{ACK}_{\mathrm{AWW}}{ }^{1}(x)$

or

$L 0: \mathrm{AW}^{0}(x, 1), \mathrm{AW}(y, 1), \mathrm{AW}^{1}(x, 2)$ fence $\mathrm{ACK} \_\mathrm{AW}(y), \mathrm{ACK}_{\mathrm{AWW}}(x), \mathrm{ACK}_{\mathrm{AWW}}{ }^{1}(x)$

(a) Asynchronous method relative execution order

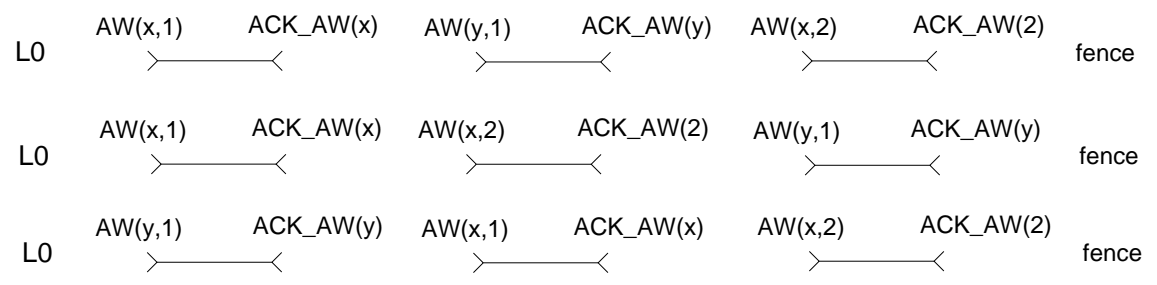

(b) Interleavings as perceived by user

Fig. 21. Asynchronous methods ordering. (a) Relative order for acknowledgments. The superscript is used to distinguish subsequent invocations of the same type on the same element/location (e.g., two writes or reads of the same variable). The or is used to denote that any interleaving is a valid one (b) Possible interleavings.

locations:

$$
\begin{aligned}
& L i: \operatorname{AW}(x, 1), \mathrm{SR}^{0}(x), \operatorname{ACK} \_\mathrm{SR}(1 \text { or } 2) \mathrm{ACK} \_\mathrm{AW}(x) \text { fence } \mathrm{SR}^{1}(x), \operatorname{ACK} \_\mathrm{SR}(a) \\
& L j: \mathrm{AW}(x, 2), \mathrm{SR}^{0}(x), \mathrm{ACK} \_\mathrm{SR}(1 \text { or } 2) \mathrm{ACK} \_\mathrm{AW}(x) \text { fence } \mathrm{SR}^{1}(x), \operatorname{ACK} \_\mathrm{SR}(a)
\end{aligned}
$$


The first read invocations $\left(S R^{0}\right)$ on both locations do not have a deterministic result. It can be either 1 or 2 and the result can be different on the two locations. After the fence, it is guaranteed that both reads $\left(S R^{1}\right)$ will return the same value $a$, though is not known if it is 1 or 2 . Assuming the element $x$ was initially zero it is guaranteed that none of the reads in the example will return 0 because of the ordering guarantees provided for accesses to the same element in a thread. The reads are always executed after the previous writes in the program order.

\section{Memory Consistency Example}

In Figure 19 we show an example of a simple STAPL program to exemplify how users can rely on the completion and ordering guarantees introduced in the previous sections to reason about the correctness of their application. For Task invocation $S 1$ can be executed concurrently with $S 4$ from Task $_{j}$. Thus the read in $S 3$ is not deterministic. The answer can be either val1 or val4. The read from S6 is deterministic and it returns val5. Invocations $S 7$ and $S 8$ respect the program order so the read in $S 9$ will return zero. After the map_func construct finishes it is guaranteed that a read of an element of the pArray will return the same value on all threads provided there is no other concurrent write in the system.

The example in Figure 19 is for illustration purposes only to describe various possible interleavings. In practice individual tasks access an exclusive set of elements from a pContainer which simplifies the reasoning about the correctness of the application. 


\section{E. Other Memory Consistency Models}

The default STAPL pContainer MCM is relaxed and similar to weak consistency $(W C)[3,23]$ discussed in the context of shared memory architectures. Under WC model there are regular memory accesses (method invocations in our case) and synchronization operations. Reordering of the operations is allowed in between synchronization points and no operation is allowed to be reordered relative to synchronization operations. As described in Sections B and C, pContainers provide additional guarantees about the ordering of the methods affecting the same data element that make the model stronger than weak consistency.

In this section we show that the default pContainer MCM is more relaxed than sequential consistency $(S C)$ or processor consistency $(P C)$ and describe how individual pContainers can restrict their interfaces to provide the requirements of these stricter models. Additionally, we show how we can further relax the ordering of the methods on the same data element to further relax the default MCM.

\section{The Default pContainer MCM is not Sequentially Consistent}

A natural memory consistency model for a parallel system is sequential consistency as introduced by Lamport[46].

Definition 13. A multiprocessor system is sequentially consistent if the result of any execution is the same as if the operations of all the processors were executed in some sequential order, and the operations of each individual processor appear in this sequence in the order specified by its program.

Using our formalism, an execution $E$ is sequentially consistent if there exists a permutation $P$ of the operations in $E$ such that 


\section{$L i: \operatorname{AW}(x, 1), \operatorname{AW}(y, 1), \operatorname{ACK} \_A W(y), \operatorname{SR}(y), \operatorname{ACK} \_S R(1)$ fence ACK_AW $(x)$}

$$
\begin{aligned}
& L 1: \operatorname{AW}(f 1,1), \operatorname{SR}(f 2), \text { ACK_SR }(0), f \text { ence, ACK_AW }(f 1) \\
& L 2: \operatorname{AW}(f 2,1), \operatorname{SR}(f 1), \text { ACK_SR }(0), f \text { ence, ACK_AW }(f 2)
\end{aligned}
$$

Fig. 22. Relaxed completion order: (a) Operations on different pContainer elements receive their acknowledgments out of order (b) Dekker's mutual exclusion.

1. For every element $x$, the restriction $P \mid x$ satisfies the specification of the data type of $x$.

2. If the acknowledgment for operation $o_{1}$ at thread $i$ occurs in $E$ before the invocation for operation $o_{2}$ at thread $i$, then $o_{1}$ appears before $o_{2}$ in $P$ (e.g., $E|i=P| i$, for all threads $i)$.

In STAPL when both synchronous and asynchronous methods are invoked from the same thread on a given location $L i$ on different elements, then there is no guarantee about the order in which these operations will be executed. In the example in Figure 22(a) we show that even though the read and write on the variable $y$ is after the asynchronous write to $x$ in the program order, the operations on $y$ may finish before the write operation on $x$ finishes.

This relaxed order for methods operating on different elements breaks the sequential consistency semantic. In Figure 22 (b) we show the Dekker mutual exclusion 
algorithm described in various memory consistency model papers [1, 2, 3]. The flags $f 1$ and $f 2$ are two elements in a pArray both of which are initially zero. Dekker's exclusion algorithm guarantees that in a SC system the reads for flags should not return both zero. Using the pArray methods however it is possible that both reads for the flags return zero due to the relaxation described in the previous paragraph.

Claim 1 : When the pContainer interface includes SM and AM methods, concurrent invocations of them on different locations do not satisfy sequential consistency.

\section{The Default pContainer MCM is not Processor Consistent}

Processor consistency $[1,2]$ guarantees that writes from a thread are seen by all other threads in the order in which they were issued. This is not guaranteed by STAPL for example in the case where the writes are on two different elements. In this situation, threads on other locations may perceive the writes on the two distinct variables in different orders. In Figure 23 we depict how threads on different locations may see the effects of two writes from location L0. Location L3 may see the element $y$ being written before element $x$ is written. This would violate the processor consistency assumptions.

Claim 2 : When the pContainer interface includes SM and AM methods, concurrent invocations of them on different locations break processor consistency.

\section{Modifying the Default pContainer MCM}

Claim 3 : When the pContainer interface includes synchronous methods only (SM), concurrent invocations of them on different locations satisfy sequential consistency.

With this constrained interface no pContainer methods are allowed to be reordered. Each method receives its acknowledgment before the next instruction in the program order is executed. This restriction, coupled with the fact that each 


$$
\begin{aligned}
& L 0: \operatorname{AW}(x, 1), \operatorname{AW}(y, 2) \ldots \ldots \ldots \ldots \ldots \ldots \ldots . . \\
& L 1: \operatorname{SR}(x) \text { ACK_SR}(1), \operatorname{SR}(y) \text { ACK_SR}(2) \\
& L 2: \operatorname{SR}(x) \text { ACK_SR }(0), \operatorname{SR}(y) \text { ACK_SR }(2) \\
& L 3: \operatorname{SR}(y) \text { ACK_SR }(2), \operatorname{SR}(x) \text { ACK_SR}(0)
\end{aligned}
$$

Fig. 23. Processor consistency counter example.

pContainer method is executed in an atomic manner, provides the necessary conditions for a SC model.

Claim 4 : The pContainer can relax its ordering constraints for methods operating on the same memory element by allowing them to proceed in an arbitrary order. With this relaxation the following interleaving is possible:

$$
L i: \operatorname{AW}(x, 1), \mathrm{SR}^{0}(x), \operatorname{ACK} \_\mathrm{SR}(0) \text { fence } \mathrm{SR}^{1}(x), \operatorname{ACK} \_\mathrm{SR}(1)
$$

Above, the synchronous read of element $x$ follows an asynchronous write but it does not return the value 1 that was previously written in the program order. After the fence, the result of the write becomes visible and the second read $\left(\mathrm{SR}^{1}\right)$ returns the value 1 .

The framework currently provides a default memory consistency model introduced in this chapter. Other more relaxed memory consistency models are possible but their impact on performance needs to be carefully analyzed and judged against 
the complexity it may bring to the user code when reasoning about correctness. Supporting stricter memory consistency models for element-wise methods is straight forward and it requires all methods to be synchronous. Applications where this will be a benefit need to be identified and analyzed.

F. pContainer Method: Developer Side

The pContainer developer expresses a method as a composition of invocations on data that may reside on different locations. When implementing a pContainer method the pContainer first decides if the element is local or not. If it is local, then the operation is performed atomically on the corresponding bContainer. Otherwise the operation is requested to be executed on a remote location. The remote location can in turn forward the execution to alternative locations in a recursive manner. In the following, we describe the semantics of the main pContainer methods in terms of requests and acknowledgments. We provide examples for read, write and split phase read operations, but the specification is the same for any element-wise pContainer method (synch, async or split phase).

Synchronous Reads (SR): A synchronous operation SR can be described as: 


$$
\begin{aligned}
& \operatorname{SR}_{L_{i}}(x), \mathrm{ACK}_{-} \mathrm{SR}_{L_{i}}(\text { val }) \equiv\left[\mathrm{SR}_{L_{i}}(m D(x))\right] / / \text { read metadata locally } \\
& ( \\
& \text { //if } x \text { is in local bContainer } \\
& \mathrm{ACK}_{\_} \mathrm{SR}_{L_{i}}\left(b \text { Cont }_{k}\right),\left[\mathrm{SR}_{L_{i}}\left(b \text { Cont }_{k}, x\right)\right], \mathrm{ACK}_{-} \mathrm{SR}_{L_{i}}(\text { val }) \\
& \text { //else } x \text { lives on location } L_{j}(\text { forwarding }) \\
& \mathrm{ACK}_{-} \mathrm{SR}_{L_{i}}\left(L_{j}\right), \mathrm{SR}_{L_{j}}(x), \mathrm{ACK}_{-} \mathrm{SR}_{L_{j}}(v a l) \mathrm{ACK} \_\mathrm{SR}_{L_{i}}(v a l) \\
& )
\end{aligned}
$$

where a $[\ldots]$ denotes an atomic execution, $L_{i}$ location $i, m D(x)$ the set of memory addresses accessed when requesting information about element $x, b$ Cont $_{k}$ bContainer owning the element $x,\left(b\right.$ Cont $\left._{k}, x\right)$ the set of memory addresses touched by the current operation on the bContainer. Everything following // is a comment.

We observe that an SR operation receives an acknowledgment before the method is finished.

Asynchronous writes (AW): An AW method is modeled similarly to a SR method except that the acknowledgment is not immediately received on the location that initiates the method. This is described next: 


$$
\begin{aligned}
& \operatorname{AW}_{L_{i}}(x) \equiv\left[\mathrm{SR}_{L_{i}}(m D(x))\right] \\
& ( \\
& / / \text { if } x \text { is in local bContainer }
\end{aligned}
$$

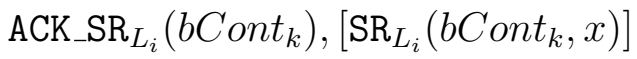

$$
\begin{aligned}
& \text { //else } x \text { lives on location } L_{j} \text { (forwarding) } \\
& \operatorname{ACK}_{-} \mathrm{SR}_{L_{i}}\left(L_{j}\right), \mathrm{AW}_{L_{j}}(x) \\
& )
\end{aligned}
$$

The acknowledgment that an $A W(x)$ operation is finished is received when encountering:

- A fence call.

- When a subsequent $\mathrm{SR}(\mathrm{x})$ or future(x).get() receives its acknowledgment.

Split phase reads (SPR): A SPR method is modeled similar to SR except that the acknowledgment is not immediately received on the location that initiates the method. This is described next: 


$$
\begin{aligned}
& \operatorname{SPR}_{L_{i}}(x), \text { Future }_{L_{i}}(x, \text { val }) \equiv\left[\mathrm{SR}_{L_{i}}(m D(x))\right] \\
& ( \\
& \operatorname{ACK} \_S R_{L_{i}}\left(b \operatorname{Cont}_{k}\right),\left[\operatorname{SR}_{L_{i}}\left(b \operatorname{Cont}_{k}, x\right)\right] \\
& \text { or } \\
& \operatorname{ACK}_{-} \mathrm{SR}_{L_{i}}\left(L_{j}\right), \operatorname{SPR}_{L_{j}}(x) \\
& ) \\
& \text { Future }_{L_{i}}(x, \text { val }) \cdot g e t() \equiv \text { ACK_SPR }_{L_{i}}(x, v a l)
\end{aligned}
$$

The Future $_{L_{i}}(x, v a l)$ is a handle that can be queried later for the acknowledgment of the SPR operation. The acknowledgment that an $S P R(x)$ operation is finished is received when encountering:

- A fence call.

- When a subsequent $\mathrm{SR}(\mathrm{x})$ receives its acknowledgment.

- When get() method of the future returned by $S P R(x)$ is invoked.

G. Consistency of Other pContainer Methods

In this section we discuss the semantics of pContainer methods that do not process individual elements. These are collective operations (e.g., constructors, destructors, etc.) and methods that return global properties of the data structure (e.g., size(), empty, etc.).

Methods such as size() or empty() for dynamic pContainers require information about the entire pContainer globally. When designing these methods, we 
considered the following alternatives: (1) a one sided reduction across all locations to perform the accumulation of all local sizes. (2) maintaining a data member size on all locations. Both these options are prohibitively expensive in terms of communication traffic generated. The solution we decided to support as part of our default implementation is to have the size stored in a replicated fashion across all locations but to update it in a lazy fashion. Dynamic operations that modify the pcontainer through inserts and deletes make the content of the size variable obsolete. The pContainer re-synchronizes the size data member upon the invocation of the post_execute() method. This is a collective call that guarantees, when finished, that pContainer size is properly reflected across all locations. The insertion of the synchronization points and calls to post_execute() is simplified by STAPL as described in the next section.

\section{H. Enforcing Synchronization Points Automatically}

We mentioned previously that acknowledgments for asynchronous methods invoked on a pContainer are not received until a synchronization point is reached. To simplify the user's effort in controlling synchronization points in STAPL, the pViews and the executor introduce them automatically while executing the tasks corresponding to a given computation. As introduced in Chapter III a computation is represented in STAPL as a collection of tasks and eventual dependencies between them. The pViews are used to represent the data stored in a pContainer and all method invocations on the container are done through pViews.

At the end of a task execution, the STAPL executor invokes a fence thus ensuring that all acknowledgments for all asynchronous methods are received before executing the next available task in the TDG. 
Additionally when all the tasks of a particular computation are finished, the post_execute() method of the pView is invoked by the executor. As part of this phase, the pViews can commit pending operations and re-synchronize the pContainer to reflect the changes done through methods in the tasks. Currently, in the post_execute() phase, pViews of dynamic pContainers update their size data member. 


\section{CHAPTER VIII}

\section{PCONTAINER PERFORMANCE EVALUATION}

In this chapter, we describe the methodology used to evaluate the performance of representative pContainers developed using the PCF: pArray, pList, pMatrix, pGraph pHashMap and composed pContainers. For all these data structures, we will look at the performance of individual methods using artificial kernels, generic algorithms and real applications.

\section{A. Experimental Setup}

We conducted our experimental studies on various parallel machines comprising various processor architectures and network interconnects. This includes a 38,288 core Cray XT4 (CRAY4), a 5312 core Cray XT5 (crayh), both available at NERSC, and a 832 core Power5 Cluster (P5-CLuster) available at Texas A\&M University. The CRAY4 has 9,572 compute nodes each with a quad core Opteron running at $2.3 \mathrm{GHz}$ and a total of $8 \mathrm{~GB}$ of memory (2 GB of memory per core). The compute nodes are connected to a dedicated SeaStar2 router through Hypertransport with a 3D torus topology which ensures low-latency, high bandwidth communication. The CRAY5 has 664 compute nodes, each containing two $2.4 \mathrm{GHz}$ AMD Opteron quad-core processors (5,312 total cores). The P5-CLUSTER is a 832 processor IBM cluster with p575 SMP nodes and 16 cores per node. In all experiments, a location contains a single processor core, and the terms can be used interchangeably. 


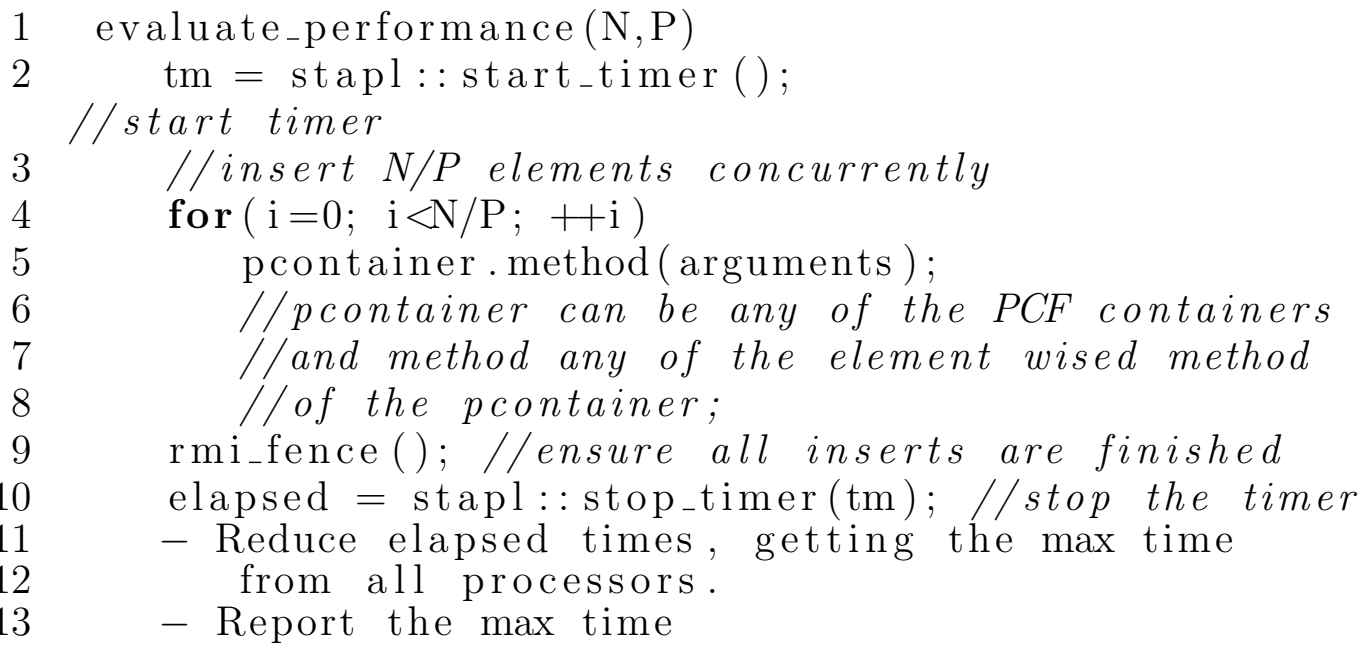

Fig. 24. Kernel used to evaluate the performance of pContainer methods.

\section{B. Evaluation of pContainer Methods}

To evaluate the scalability of individual methods we designed the kernel shown in Figure 24. The figure shows a generic method being invoked, and the same kernel is used to evaluate all methods. For a given number of elements $N$, all $P$ available processors (locations) concurrently perform $N / P$ method invocations. We report the time taken to perform all $N$ methods globally. The measured time includes the cost of a fence call which, as stated in Chapter III, is more than a simple barrier. Each experiment is executed 32 times on P5-CLUSTER and CRAY4, and 10 times on CRAY4 due to large scale experiments performed there and limited execution time available on the machine. The times reported in the graphs in the following chapters are average times with confidence intervals. Due to the stability of the machine the confidence intervals are small for certain experiments and are not always visible in the graphs. 


\section{Evaluation of Generic Algorithms}

We evaluated the performance of generic non-mutating pAlgorithms, p_generate, p_for_each and p_accumulate, when applied to data stored in various different STAPL pContainers. For all the algorithms considered in this section, for all platforms, we conducted weak scaling experiments. Strong scaling would be difficult to evaluate due to the short execution times of the algorithms even when run on very large input sizes. Additionally, when scalling to very large number of processors the problem would not fit in memory for lower processor counts.

The p_generate algorithm takes as argument a functor that generates a random number and a pView. It assigns a random number to every element in the pView. The p_for_each algorithm increments the elements of the container with a given constant performing read, add and store operations on each individual element without the need of any remote accesses. The p_accumulate accumulates all the elements in the container using a generic map reduce operation available in STAPL. These three algorithms are representative of a large class of algorithms that are either map or map reduce patterns.

\section{Specific Applications}

For various pContainers we look at the performance in the context of more complex applications. These are examples of how programmers may use pContainers in applications. 


\section{CHAPTER IX}

THE STAPL PARRAY

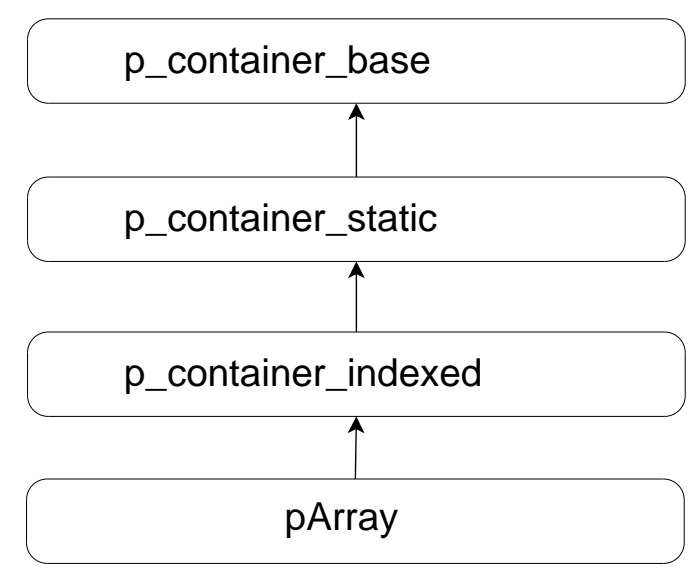

Fig. 25. Derivation chain for pArray.

The STL valarray container is a fixed size data structure optimized for storing and accessing data based on one dimensional indices and iterators. The STAPL pArray is the parallel equivalent of the STL valarray, providing an efficient interface to access data elements using indices and pViews. An important property of the pArray is that it is a static data structure, i.e., the number of elements is known at instantiation and doesn't change during execution. As described in Chapter V, Section A, this enables a number of optimizations such as closed form solutions for partitions and partition mappings. In Figure 25, we show the derivation chain for the pArray container. Correspondingly, the pArray inherits the interfaces and the default functionality provided by all its base classes. In Chapter V, Section E, we introduced the 
default pArray specification for the main functional modules. In this section we show a simple example of pArray usage, provide the user interface and discuss experimental results.

A. Example

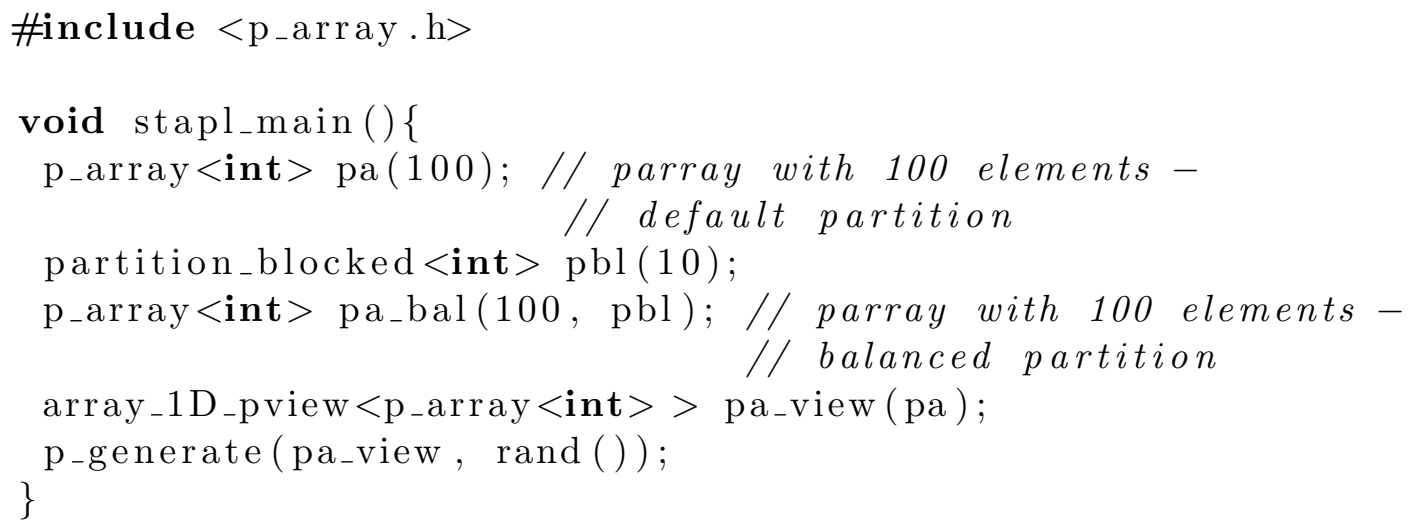

Fig. 26. pArray example.

In Figure 26 we show a simple example of pArray usage. The program declares a pArray of 100 integers (Figure 26, Line 4) and another pArray of 100 integers with a blocked partition with blocks of size 10 (Figure 26, Line 7). A pView is declared next over a pArray (Figure 26, Line 9) and a generic algorithm is invoked over the data of the pArray(Figure 26, Line 10). 
B. The pArray Specification

The pArray template declaration is :

template $<$ class $T$, class Partition=Default, class Traits=Default $>$ class p_array;

We include in Table XIX the complete interface of the pArray.

Table XIX.: pArray interface.

\begin{tabular}{|c|c|}
\hline Template Arguments & Description \\
\hline $\mathrm{T}$ & $\begin{array}{l}\text { The array's value type: the type of object } \\
\text { that is stored in the array }\end{array}$ \\
\hline Partition & $\begin{array}{l}\text { Partition used to define the blocks which the } \\
\text { pArray is divided }\end{array}$ \\
\hline Traits & $\begin{array}{l}\text { pArray traits for specifying the low level } \\
\text { base container used and distribution fea- } \\
\text { tures }\end{array}$ \\
\hline Define Type & Description \\
\hline value_type & The type of stored objects. \\
\hline index_type & The type of the indices of pArray \\
\hline Method & Description \\
\hline p_array () & Default constructor. $O(\log (P))$ \\
\hline p_array (size_t m) & $\begin{array}{l}\text { Create a p_array of } m \text { elements. } O(m / P+ \\
\log (P))\end{array}$ \\
\hline p_array(size_t m, value_type _val) & $\begin{array}{l}\text { Create a p_array of } m \text { elements and initialize } \\
\text { the elements to _val. } O(m / P+\log (P))\end{array}$ \\
\hline p_array (const p_array\& other) & $\begin{array}{l}\text { Copy constructor. } \quad O(\text { size }(\text { other }) / P+ \\
\log (P))\end{array}$ \\
\hline $\begin{array}{l}\text { template <class PS> } \\
\text { p_array (size_t m, const PS\&) }\end{array}$ & $\begin{array}{l}\text { Construct a p_array of a given size and using } \\
\text { the specified partition. } O(m / P+\log (P))\end{array}$ \\
\hline $\begin{array}{l}\text { void set_element ( index_type i, } \\
\text { const value_type\& v) }\end{array}$ & $\begin{array}{l}\text { Set the element of index } i \text { to the value } \mathrm{v} \text {. } \\
O(1)\end{array}$ \\
\hline $\begin{array}{l}\text { value_type get_element ( index_type } \\
\text { i) const }\end{array}$ & $\begin{array}{l}\text { Return the value corresponding to index } i \text {. } \\
O(1)\end{array}$ \\
\hline
\end{tabular}


Table XIX continued

\begin{tabular}{|c|c|}
\hline Method & Description \\
\hline $\begin{array}{l}\text { pc_future <value_type }> \\
\text { get_element_split (const index_type\& } \\
\text { i) const }\end{array}$ & $\begin{array}{l}\text { Returns immediately (non blocking) a fu- } \\
\text { ture for the value corresponding to index } \\
\text { i. The future will return the actual value } \\
\text { when queried using the corresponding get() } \\
\text { method. } O(1)\end{array}$ \\
\hline $\begin{array}{l}\text { template<class Functor }>\text { typename } \\
\text { Functor: :result_type apply_get } \\
\text { (index_type i, Functor f) }\end{array}$ & $\begin{array}{l}\text { Apply a functor } f \text { to the data corresponding } \\
\text { to index } i \text {. The returned value is the value } \\
\text { returned by functor } f . O(1)\end{array}$ \\
\hline $\begin{array}{l}\text { template<class Functor> void } \\
\text { apply_set (index_type i, Functor } \\
\text { f); }\end{array}$ & $\begin{array}{l}\text { Apply a functor } f \text { to the data corresponding } \\
\text { to index i. } O(1)\end{array}$ \\
\hline $\begin{array}{l}\text { p_container_indexed_ref operator }[] \\
\text { (index_type i) }\end{array}$ & $\begin{array}{l}\text { Access operator that returns a reference to } \\
\text { element i. } O(1)\end{array}$ \\
\hline $\begin{array}{l}\text { template <class NPS> void } \\
\text { set_partition(const NPS\& ps) }\end{array}$ & $\begin{array}{l}\text { Reset the partition of the pArray to be ps. } \\
O(\text { size } / P+\log (P))\end{array}$ \\
\hline bool is_local (index_type i) & $\begin{array}{l}\text { Return true if the element with index } i \text { is } \\
\text { stored locally, false if it is remote. } O(1)\end{array}$ \\
\hline
\end{tabular}

C. pArray Partitions

The pArray partitions model the partition_indexed concept. Users can provide their own partitions or select among the ones included in the table XX.

Table XX.: pArray partitions.

\begin{tabular}{|l|l|}
\hline Partition Type & Description \\
\hline partition_balanced $<\mathrm{T}>$ & $\begin{array}{l}\text { Partition the pArray into evenly sized } \\
\text { ranges; } \mathrm{T} \text { is the p_array value type }\end{array}$ \\
\hline partition_blocked<T> (size_t bs) & $\begin{array}{l}\text { Split the pArray of size } \mathrm{n} \text { into } \\
\text { ceiling }(\mathrm{n} / \mathrm{bs})-1 \text { blocks of size } \\
\mathrm{bs} \text { the last block will have size } \\
\mathrm{n}-(\text { ceiling }(\mathrm{n} / \mathrm{bs})-1) * \mathrm{bs}\end{array}$ \\
\hline
\end{tabular}


Table XX continued

\begin{tabular}{|l|l|}
\hline Partition Type & Description \\
\hline partition_blocked_explicit<T> & Partition a pArray into blocks whose sizes \\
(const std: :vector<size_t> sizes) & are defined in the vector sizes (the sum of \\
& the sizes have to be equal to the size of the \\
& pArray \\
\hline
\end{tabular}

D. pArray Customization

Low level details about a pArray data structure are customized using the traits mechanism. In Table XXI we describe the modules that are customizable.

Table XXI.: pArray traits.

\begin{tabular}{|l|l|}
\hline bContainer & pArray storage \\
\hline partition_type & pContainer partition \\
\hline mapper_type & $\begin{array}{l}\text { Class to specify the mapping of the } \\
\text { bContainers into locations }\end{array}$ \\
\hline distribution_type & Data distribution manager \\
\hline iterators_type & Type of iterators exported by pContainer \\
\hline loc_dist_metadata & $\begin{array}{l}\text { Class used to specify pContainer coarsen- } \\
\text { ing information }\end{array}$ \\
\hline
\end{tabular}

The partition type can be any of the ones described in the Table XX. The partition mapper type can be any of the partition_mapper_generic, partition_mapper blocked, partition_mapper_cyclic or partition_mapper_identity. iterators_type is a struct defining the iterator, const iterator, reference and const reference (global iterator and reference). The default implementation for pArray iterators 
is the pc_iterators class that provides a generic random access iterator. The loc_dist_metadata is required by pView to extract the pContainer locality metadata. The default provided implementation for this module is a class that specifies to the pView to create a sub view for each bContainer.

\section{E. Performance Evaluation}

We evaluate the performance of the pArray parallel methods and STL algorithms operating on data stored inside pArrays. To evaluate the performance of the methods, we use weak scaling experiments according to the methodology described in Chapter VIII.

CRAY: pArray Construction Weak Scaling $10,20,50 \mathrm{M}$ elements/location

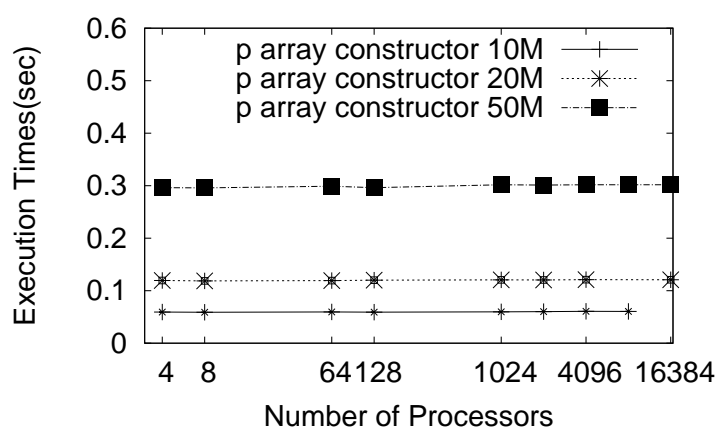

(a) pArray constructor on CRAY4
P5: pArray Constructor Weak Scaling 10, 20, 50M elements/location

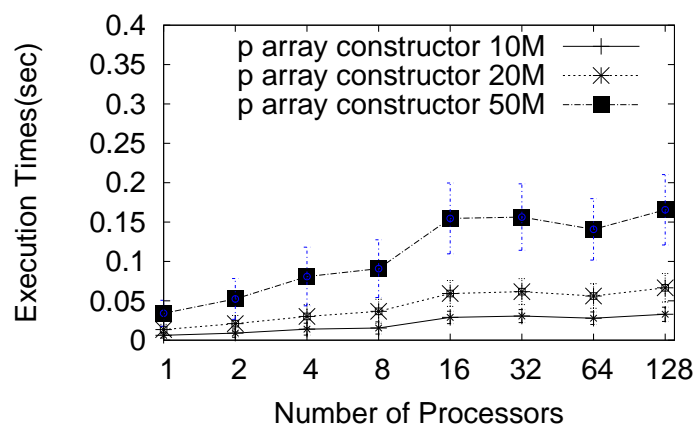

(b) pArray constructor on P5-CLUSTER

Fig. 27. pArray constructor execution time for various input sizes on (a) CRAY4 and (b) P5-CLuster. 


\section{Methods}

In this section we analyze the performance of pArray collective operations (e.g., constructors) and representative element-wise methods set_element, get_element and split_phase_get_element. In a first experiment included in Figure 27 we show the performance of the pArray constructor on two different parallel architectures for 10, 20 and 50 million elements per location. The pArray constructor is a fully parallel operation with none of its modules requiring global synchronizations. On CRAY4 (Figure 27(a)) we observe very good scaling from 4 processors (the size of a compute node) up to 16384 . When using $50 \mathrm{M}$ elements per location the total size of the allocated pArray on 16384 processors is 819.2 billion requiring 1.19 terabytes of storage. On P5-Cluster (Figure 27(b)) we observe the performance slowly degrading as we increase the number of processors from 1 to 16, which uses up a shared memory node of the cluster. From 16 processors up the scaling improves. This is a well known behavior on this architecture and it is due to memory bandwidth limitations within a shared memory node as we use an increasing number of processors.

In Figure 28, we show the scalability of pArray set_element and get_element for various input sizes and numbers of invocations. The number of method invocations is the same as the input size $N$ and each location performs concurrently $N / P$ invocations, where $P$ is the number of locations. The times in this figure are for the case when all invocations are executed locally. In this situation, both methods scale well as they don't involve any additional communication. In Figure 29, we show in the same plot the pArray constructor, set_element and get_element, respectively, to see the relative performance difference of these methods. set_element is an asynchronous method with no return type, while the get_element returns the value read. For this reason, the set_element is faster than get_element even though all invocations are 


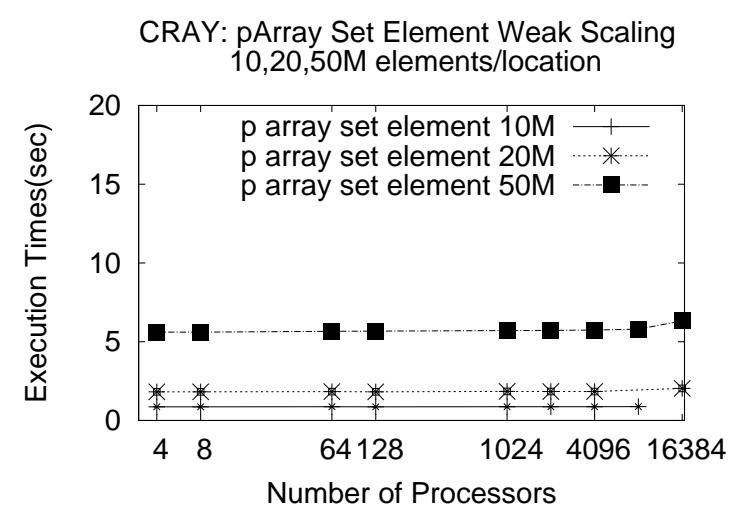

(a) pArray set_element

P5: pArray SetElement Weak Scaling 10, 20, 50M elements/location

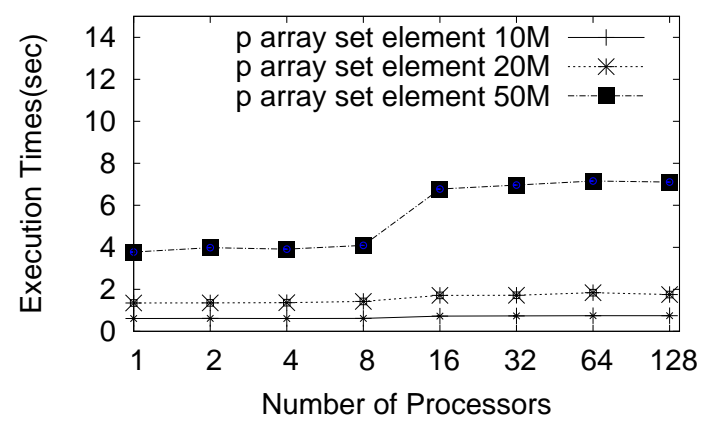

(c) pArray set_element

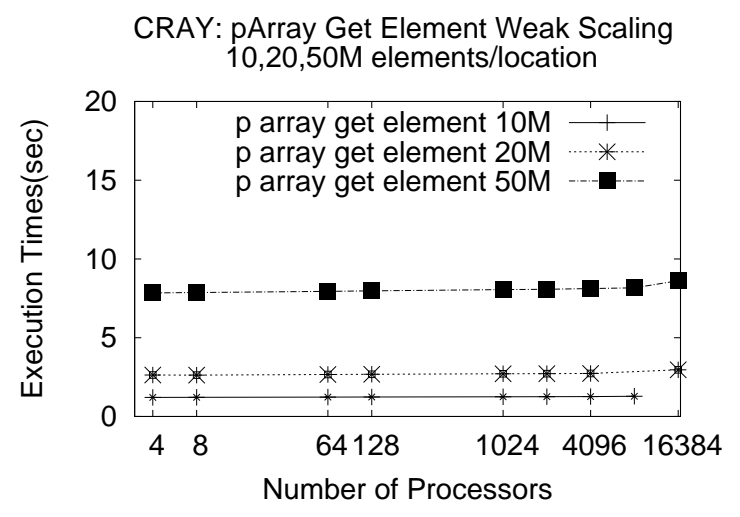

(b) pArray get_element

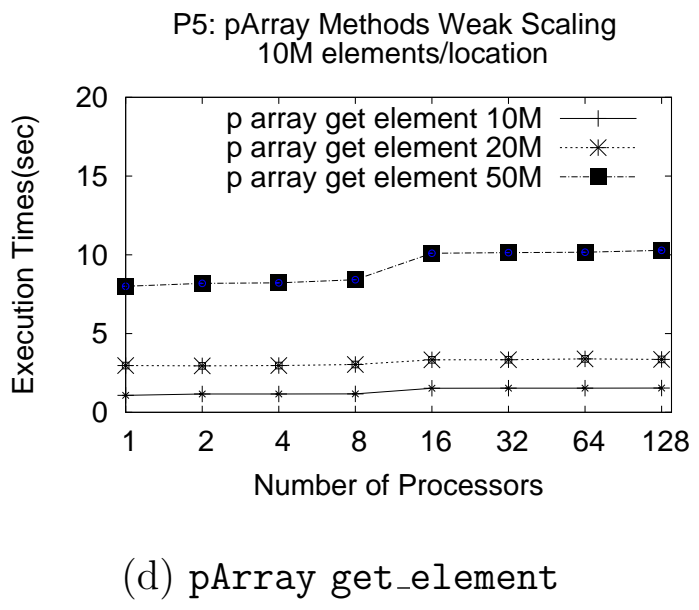

Fig. 28. CRAY4: pArray local method invocations for various container sizes. The number of invocations is the same as the pArray size (a) set_element (b) get_element.

local.

Split phase method study: In this experiment we study the performance of the pArray methods when there are $1 \%$ remote accesses. The results are included in Figure 30. We observe good scalability with only a $5.8 \%$ execution time increase for the asynchronous invocations as we scale the number of processors from 


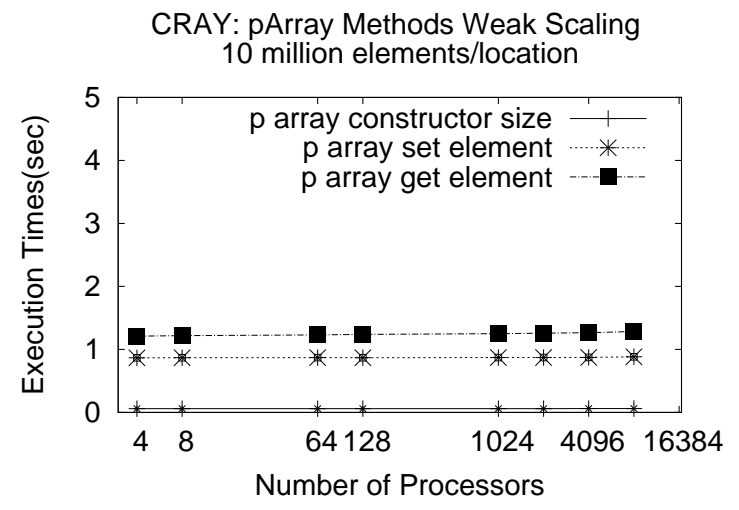

(a) $10 \mathrm{M}$ elements per location

CRAY: pArray Methods Weak Scaling 50 million elements/location

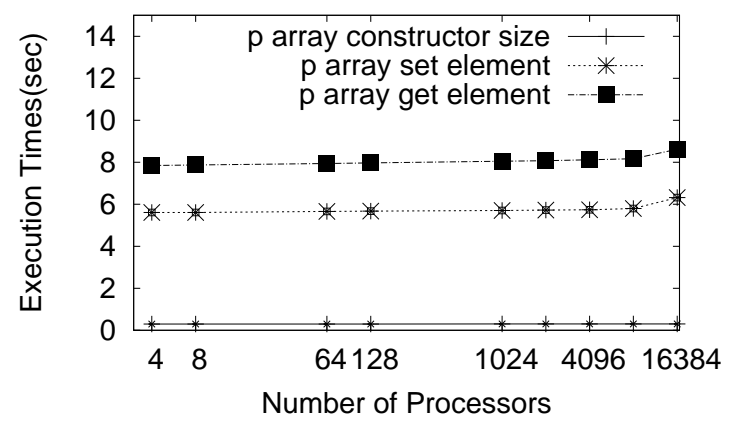

(b) 50M Elements per location

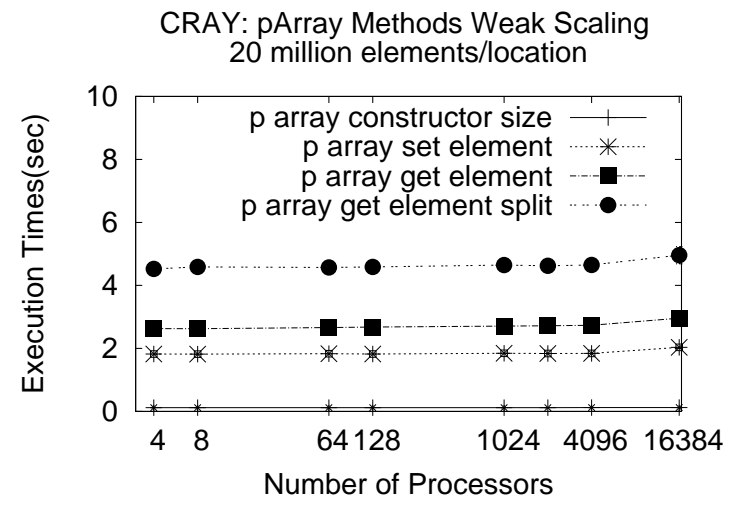

(b) 20M Elements per location

Fig. 29. CRAY4: pArray methods for various input sizes. All invocations are local. 


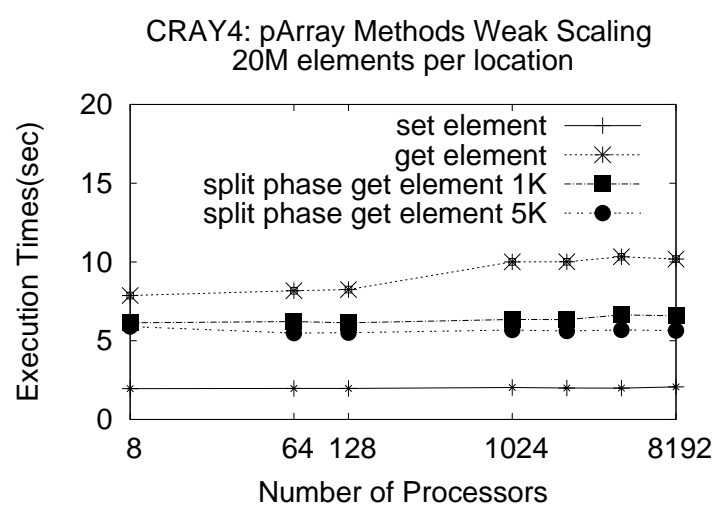

Fig. 30. CRAY4: pArray methods set_element, get_element and split phase get_element. $20 \mathrm{M}$ method invocations per location with $1 \%$ remote.

8 to 8192 . For the synchronous methods the execution time increases $29 \%$. For the split_phase_get_element we performed two experiments where we invoke groups of 1000 or 5000 split phase operations before waiting for them to complete. The split phase methods have an inherent overhead for allocating the futures on the heap but they do enable improved performance and scalability relative to the synchronous methods. Split phase execution enables the aggregation of the requests in the runtime as well as allowing communication computation overlap. For the split_phase_get_element the overall execution time increases $7.1 \%$ and $4.5 \%$ when 1000 and 5000 invocations, respectively, are started before waiting the result.

Remote methods study: In this experiment we study the performance of the pArray element-wise methods for various percentages of remote invocations. The results for the two architectures considered are included in Figure 31. The percent of remote method invocations is either $2 \%$ or $5 \%$. As we increase the number of methods that are executed remotely, the execution time for all methods increases correspondingly. The set_element scales well on both architectures as we increase the 


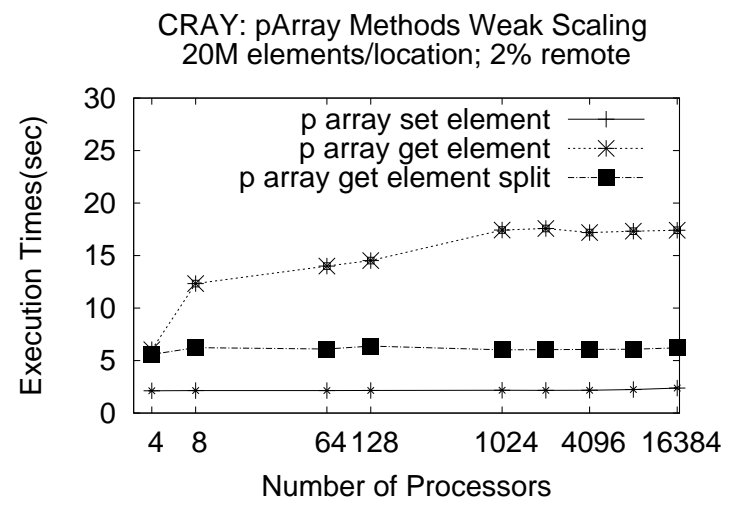

(a) $2 \%$ remote

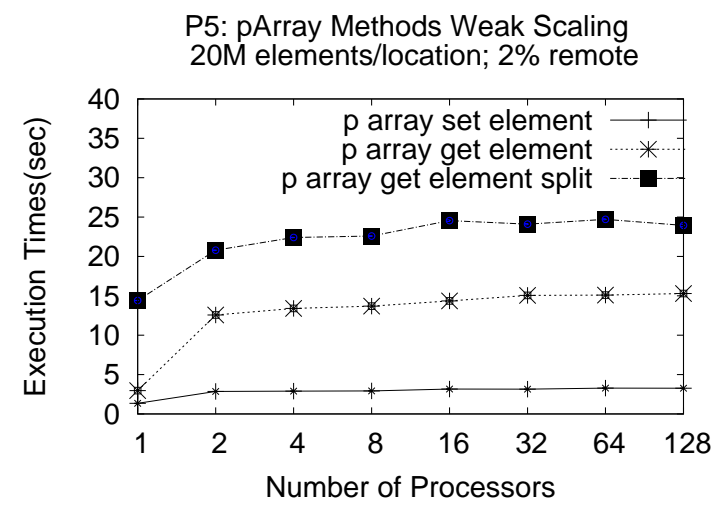

(c) $2 \%$ remote

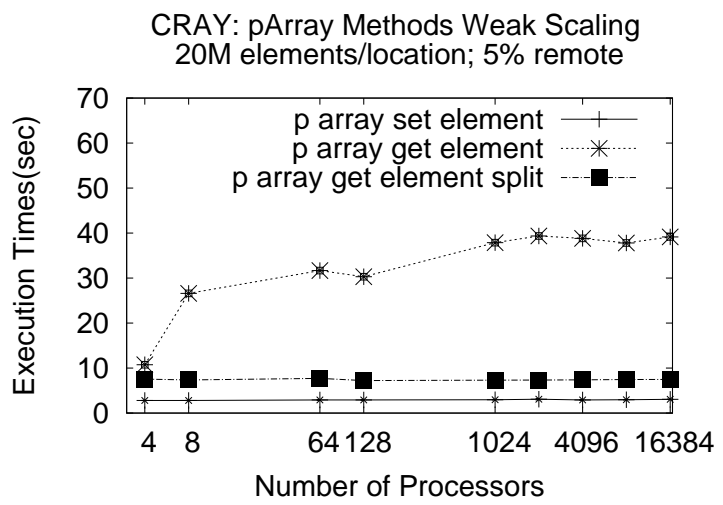

(b) $5 \%$ remote

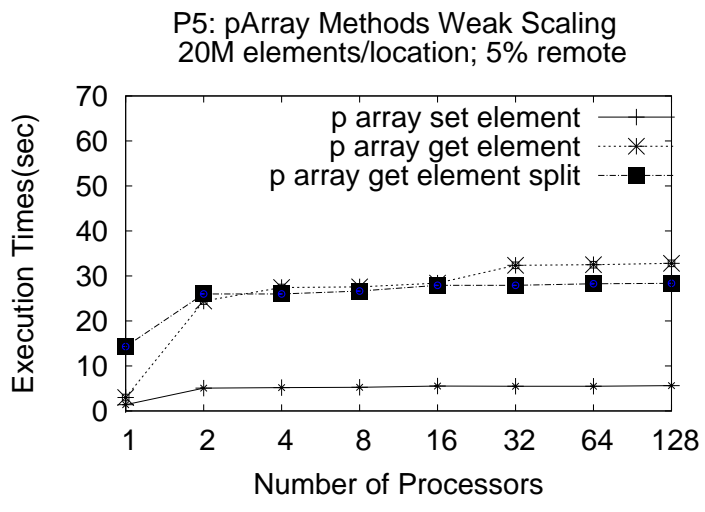

(d) $5 \%$ remote

Fig. 31. pArray methods for various percentage of remote invocations. Execution times for (a)(b) CRAY4 and (c)(d) P5-CLUSTER. 


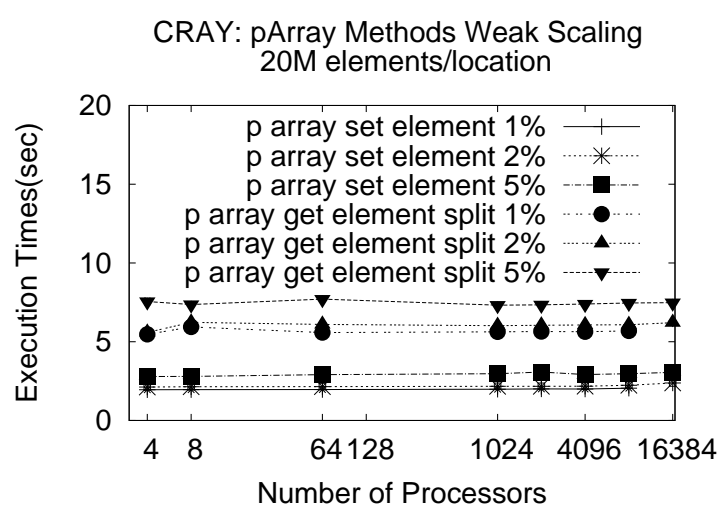

(a)

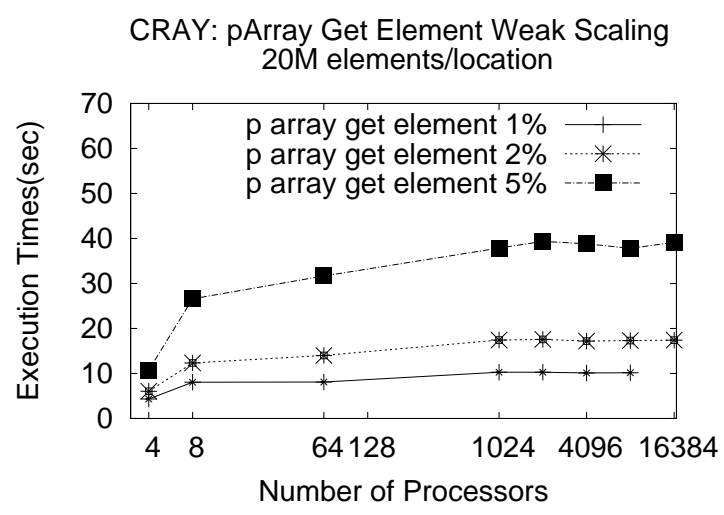

(b)

Fig. 32. CRAY4: pArray local and remote method invocations for various container sizes. The number of invocations is the same as the pArray size. The number of remote invocations is varied from $1 \%$ to $5 \%$ (a) set_element and split_phase_get_element (b) get_element.

number of processors. For the split_phase_get_element we aggregate 5000 methods before waiting for their completion. On CRAY4 this operation scales well and overall is faster that the synchronous get_element. On the P5-CLUSTER architecture, the overhead of allocating the futures for the return values is bigger than on CRAY4 and on this platform the benefits of split phase execution are visible only when the number of remote methods is $5 \%$. Overall, we observe that the asynchronous and split phase methods scale better than the synchronous methods but all methods considered scale well up to 16384 processors.

In Figure 32, we show for CRAY4 the impact of increasing the remote method invocations for individual methods. We observe that the runtime increases for all methods. The set_element and split_phase_get_element scale well while increasing the number of processors while the get_element performs slightly worse. 


\section{Algorithms}

In Figure 33(a), we show the execution times for the pAlgorithms p_generate, p_for_each and p_accumulate on pArray. The figure shows a weak scaling experiment with 20M elements per processor. From the plot we observe that for the pArray the performance degradation is $5 \%$ when scaling from 128 to 16,385 processors for p_generate and p_for_each. For p_accumulate, which performs a global reduction to provide the result, the performance degradation is about $33 \%$. This is due to the limited amount of computation performed to access and add local elements relative to the communication cost of the reduction. Figure 33(b) shows a generic inner product algorithm operating on the data in two pArrays. For two given pArrays $A[N]$ and $\mathrm{B}[\mathrm{N}]$ the algorithm computes res $=\sum_{i=0 . . N-1}(A[i] * B[i])$. the complexity of the parallel algorithm is $O(N / P+\log (P))$ where $N$ is the number of elements and $P$ is the number of available locations.

A more complex algorithm that we analyze in this section is the string matching. It takes as input two pViews defined over two pContainers and counts the number of occurrences of the data of the second pView (e.g., pattern) in the first one (e.g., sequence). This algorithm incurs communication that can be at most the size of the pattern. In Figure 33(c) we include results for two weak scaling experiments. In a first experiment, we search for a pattern that doesn't exist in the input sequence. The input sequence is filled with "A" and the pattern is "ZZZ". In this case there is no communication. In a second scenario we search for the pattern "AAA" that occurs $N-2$ times in the input sequence. As we see from the figure, this computation scales well as we scale the number of processors from 4 to 16384. 


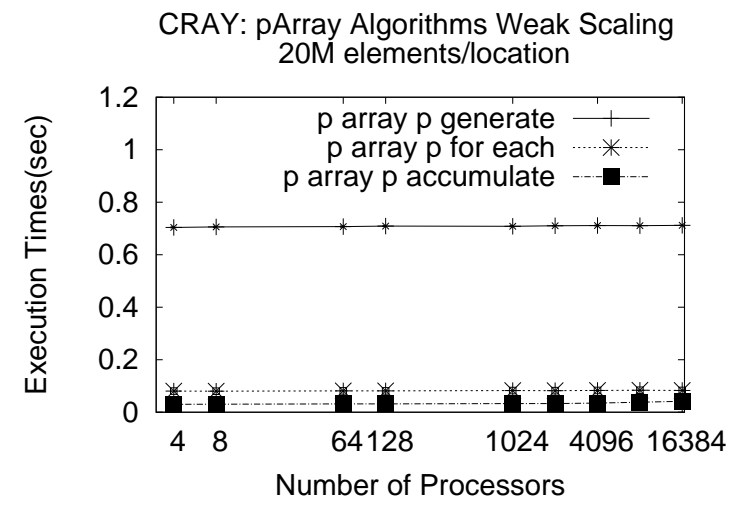

(a) p_generate, p_for_each, p_accumulate

CRAY: String matching alg. weak scaling $10 \mathrm{M}$ chars. per location

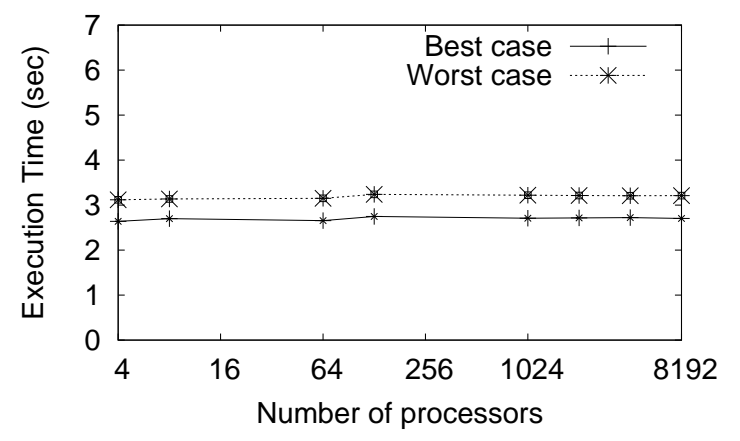

(c) String matching

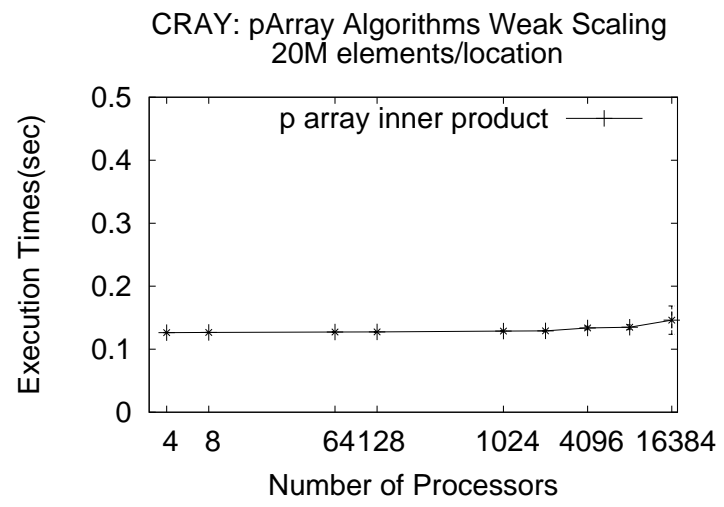

(b) Inner product

Fig. 33. Execution times for generic algorithms on CRAY4 for a pArray with 20M elements per processor. 


\section{F. Memory Consumption Study}

In this section we analyze the memory consumption of the pArray. We analyze this using pContainer internal methods that report memory usage and using the Integrated Performance Monitoring (IPM) [62] tool available for CRAY4. All memory sizes reported in this section are in MB.

Table XXII.: pArray memory consumption: The first two columns shows the IPM reported memory usage for a simple STAPL program that performs only one fence. The next two columns shows the memory usage for a STAPL program that declares an std: : valarray of 20M integers on each location; The last two columns shows the memory usage for a STAPL program that declares a pArrayof size $\mathrm{P}^{*} 20 \mathrm{M}$ integers. All sizes are in $\mathrm{MB}$

\begin{tabular}{|r|r|r|r|r|r|r|}
\hline & \multicolumn{2}{|c|}{ stapl_main } & \multicolumn{2}{|c|}{ stap_main and vallarray } & \multicolumn{2}{|c|}{ stapl_main and pArray } \\
\hline Processors & Total & PerLoc & Total & PerLoc & Total & PerLoc \\
\hline 4 & 155.72 & 38.93 & 460.89 & 115.22 & 461.14 & 115.29 \\
\hline 8 & 838.96 & 104.89 & 1449.3 & 181.19 & 1449.8 & 181.25 \\
\hline 64 & 6721.94 & 105.08 & 11604.68 & 181.37 & 11608.78 & 181.5 \\
\hline 128 & 13477.89 & 105.3 & 23243.37 & 181.59 & 23251.35 & 181.66 \\
\hline 1024 & 110880.77 & 108.29 & 189004.8 & 184.59 & 189310.98 & 185.58 \\
\hline 2048 & 229049.34 & 111.91 & 385298.43 & 188.21 & 386081.79 & 190.33 \\
\hline 4096 & 487207.94 & 118.97 & 799720.45 & 195.26 & 802568.19 & 199.66 \\
\hline 8192 & 1088993.28 & 133.05 & 1714012.16 & 209.35 & 1724835.84 & 218.73 \\
\hline
\end{tabular}

In Table XXII, column 3 and 4, we include the memory usage as reported by IPM for a simple STAPL program that only performs a fence and exits. IPM reports the maximum memory used (watermark) across all processes of the application. We observe that even though there is no data allocated by the program, the memory used is still considerable. This is mainly due to the communication and synchronization buffers allocated by the underlying run time system and MPI. The maximum mem- 
ory used per processor increases with the number of processors from $38.93 \mathrm{MB}$ on 4 processors to $133.05 \mathrm{MB}$ on 8192 . A considerable jump happens from 4 to 8 processors when inter-node communication is required.

In Table XXII, columns 4 and 5, show the memory usage as reported by IPM when a sequential array of integers of size 20 million is allocated inside STAPL main. Columns 6 and 7 show the memory used when STAPL main declares a pArray with $20 \mathrm{M}$ elements per location. We notice very similar memory usage as we increase the number of processors for these two situations. The pArray does allocate slightly more memory than a simple valarray to store the data distribution information, the location manager and the infrastructure required for synchronization (e.g., thread safety manager). In Table XXIII we show the theoretical minimum memory used by a distributed array with $20 \mathrm{M}$ elements per location. This is computed as $20 M * P *$ sizeof (int).

Table XXIII.: Theorethical memory usage for pArray: minimum required memory (no meta data) and pArray reported memory consumption (data and meta data). All sizes are in $\mathrm{MB}$

\begin{tabular}{|r|r|r|r|r|}
\hline & \multicolumn{2}{|c|}{ Theoretical } & \multicolumn{2}{c|}{ pArray reported } \\
\hline Processors & Total & PerLoc & Total & PerLoc \\
\hline 4 & 305.176 & 76.294 & 305.178 & 76.295 \\
\hline 8 & 610.352 & 76.294 & 610.357 & 76.295 \\
\hline 64 & 4882.813 & 76.294 & 4882.850 & 76.295 \\
\hline 128 & 9765.625 & 76.294 & 9765.700 & 76.295 \\
\hline 1024 & 78125.000 & 76.294 & 78125.600 & 76.295 \\
\hline 2048 & 156250.000 & 76.294 & 156251.000 & 76.295 \\
\hline 4096 & 312500.000 & 76.294 & 312503.000 & 76.295 \\
\hline 8192 & 625000.000 & 76.294 & 625005.000 & 76.295 \\
\hline
\end{tabular}




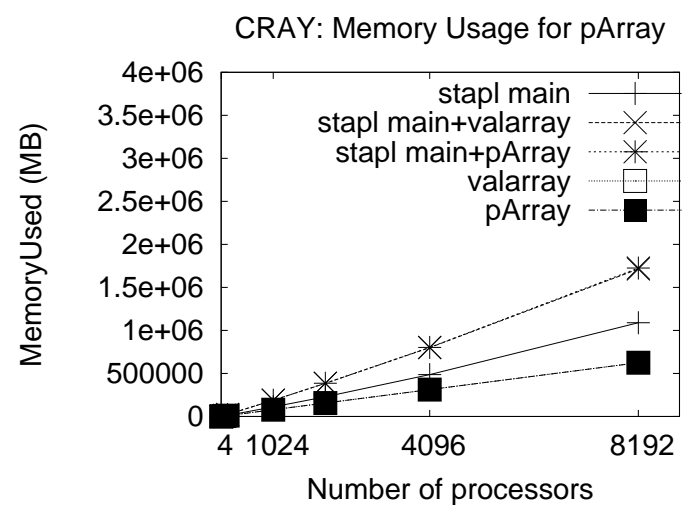

(a) Total across processors

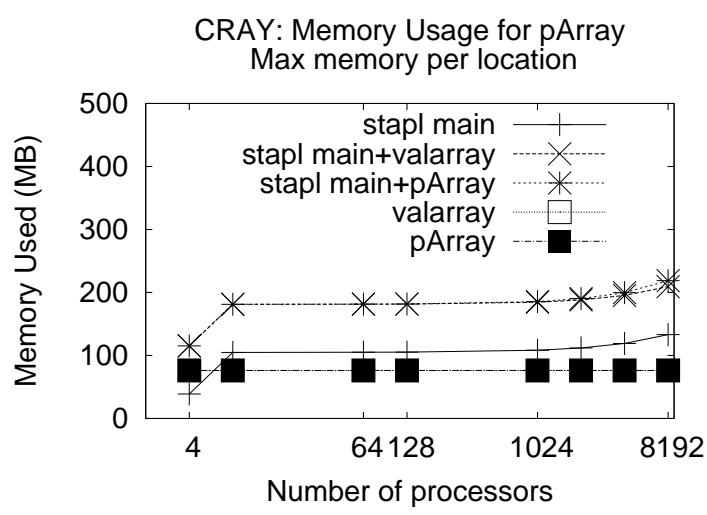

(b) Memory used per location

Fig. 34. CRAY4: pArray memory usage study. The simple program contains either no data (stapl main), a valarray of 20M elements per location (stapl main+valarray), a pArray of size $\mathrm{P}^{*} 20 \mathrm{M}$ (stapl main+pArray). Theoretical memory usage for a valarray of size $\mathrm{P}^{*} 20 \mathrm{M}$ (valarray) and pArray reported memory usage (pArray). Lines 2,3 overlap and similar lines 4,5. (a) Total memory used; (b) Memory used per location.

In Figure 34(a), we plot the data from Tables XXII and XXIII. We observe the memory per location increasing for all situations measured with IPM. This is an expected trend that is accordingly documented in the MPI user manual of the machine. In Figure 34(b), we show the memory used per location. We notice a slight difference between the STAPL program declaring a valarray and the STAPL program declaring a pArray. The difference increases with the number of processors from $0.06 \%$ on 4 processors to $4 \%$ on 8192 processors. 


\section{CHAPTER X}

\section{THE STAPL PLIST*}

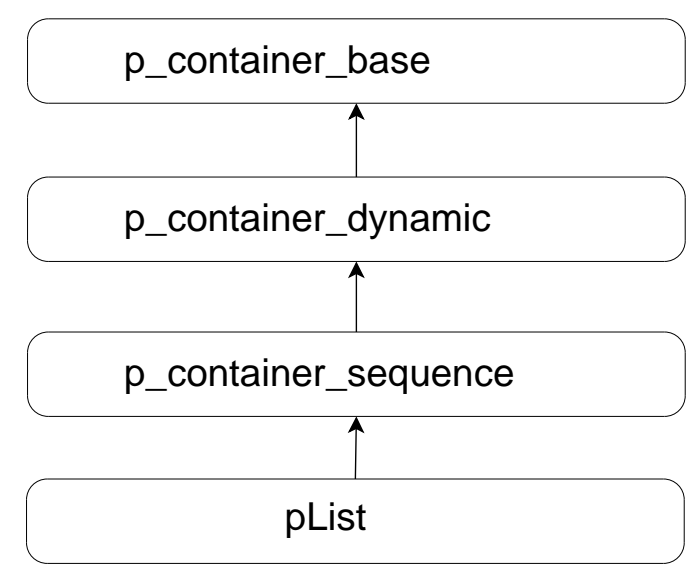

Fig. 35. Derivation chain for pList.

The linked list is a fundamental data structure that plays an important role in many areas of computer science and engineering such as operating systems, algorithm design, and programming languages. A large number of languages and libraries provide different variants of lists with $\mathrm{C}++$ STL being a representative example. The STL list is a generic dynamic data structure that organizes the elements as a sequence and allows fast insertions and deletions of elements at any point in the sequence. The

*Part of the data reported in this chapter is reprinted with the kind permission of Springer Science+Business Media from "The STAPL pList" by G. Tanase, X. Xu, A. Buss, Harshvardhan, I. Papadopoulos, O. Pearce, T. Smith, N. Thomas, M. Bianco, N. M. Amato, and L. Rauchwerger, 2009. Lecture Notes in Computer Science, vol. 5898, pp. 16-30, Copyright 2009 by Springer. 
STAPL pList is a parallel equivalent of the STL list with an interface for efficient insertion and deletion of elements in parallel. the derivation chain for pList is included in Figure 35.

Most STL equivalent methods require a return value, which in general translates into a blocking method. For this reason, we provide a set of asynchronous methods, e.g., insert_async and erase_async. These non-blocking methods allow for better communication/computation overlap and enable the STAPL RTS to aggregate messages to reduce the communication overhead. Since there is no data replication, operations such as push_back and push_front, if invoked concurrently, may produce serialization in the locations managing the head and the tail of the list. For this reason, we added two new methods to the pList interface, push_anywhere and push_anywhere_async, that allow the pList to insert the element in an unspecified location in order to minimize communication and improve concurrency.

\section{A. pList Example}

We include in Figure 36 a simple example that inserts all elements from an input pArray into a pList using their corresponding pViews. The program declares a pArray of size 1000 and an empty pList using their default partitions and traits (Figure 36, Lines 11 and 12). Subsequently, in line 14 the STAPL map_func construct is called to create tasks that take individual elements from the pArray and insert them into the pList. The work function of the tasks created by map_func is included starting with line 4 and it invokes the push_anywhere method of pList.

\section{B. pList Specification}

The pList declaration is: 


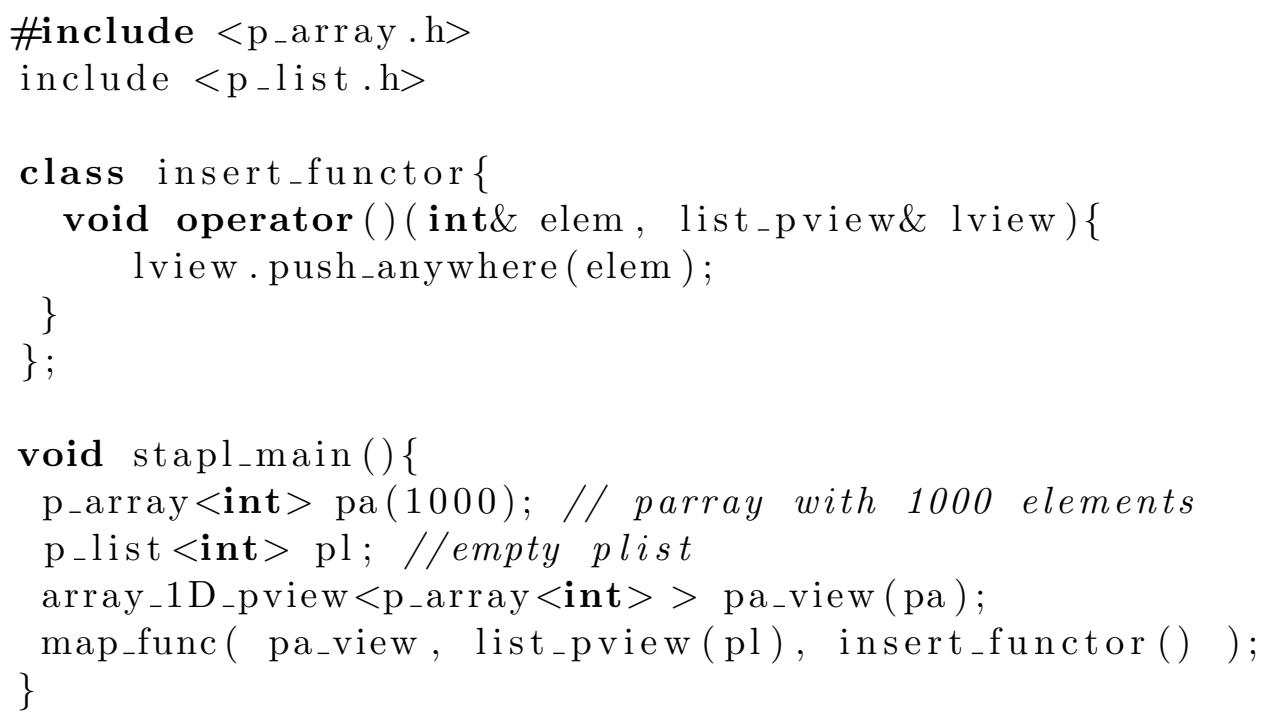

Fig. 36. pList example.

template $<$ class $T$, class Partition=Default, class Traits=Default $>$ class p_list;

The pList has the derivation chain included in Figure 35. We include in Table XXIV the pList interface.

Table XXIV.: pList interface.

\begin{tabular}{|l|l|}
\hline Method & Description \\
\hline p_list(size_t N, const T\& value $=\mathrm{T}())$ & $\begin{array}{l}\text { Creates a pList with N elements, each of } \\
\text { which is a copy of value. } O(N / P+\log (P))\end{array}$ \\
\hline p_list(size_t N, partition_type\& ps) & $\begin{array}{l}\text { Creates a pList with N elements based } \\
\text { on the given partition strategy. } O(N / P+ \\
\log (P))\end{array}$ \\
\hline void splice(iter pos, pList\& pl); & $\begin{array}{l}\text { Splice the elements of pList pl into the cur- } \\
\text { rent list before the position pos. }\end{array}$ \\
\hline size_t size() const & Returns the size of the pList. $O(\log (P))$ \\
\hline bool empty() const & True if the pList's size is $0 . O(\log (P))$ \\
\hline
\end{tabular}


Table XXIV continued

\begin{tabular}{|l|l|}
\hline Method & Description \\
\hline T\& [front|back]() & $\begin{array}{l}\text { Access the first/last element of the se- } \\
\text { quence. } O(1)\end{array}$ \\
\hline void push_[front|back](const T\& val) & $\begin{array}{l}\text { Insert a new element at the beginning/end } \\
\text { of the sequence. } O(1)\end{array}$ \\
\hline void pop_[front|back]() & $\begin{array}{l}\text { Remove the element from the begin- } \\
\text { ning/end of the sequence. } O(1)\end{array}$ \\
\hline iterator insert(iterator pos, const T\& val) & $\begin{array}{l}\text { Insert val before position pos and return the } \\
\text { iterator to the new inserted element. } O(1)\end{array}$ \\
\hline $\begin{array}{l}\text { void insert_async(iterator pos, const T\& } \\
\text { val) }\end{array}$ & $\begin{array}{l}\text { Insert val before pos with no return value. } \\
O(1)\end{array}$ \\
\hline iterator erase(iterator pos) & $\begin{array}{l}\text { Erases the element at position pos and re- } \\
\text { turns the iterator pointing to the new loca- } \\
\text { tion of the element that followed the element } \\
\text { erased. } O(1)\end{array}$ \\
\hline void erase_async(iterator pos) & $\begin{array}{l}\text { Erases the element at position pos with no } \\
\text { return value. } O(1)\end{array}$ \\
\hline $\begin{array}{l}\text { iterator push_anywhere(const value_type\& val on to the last local bContainer } \\
\text { val) }\end{array}$ & $\begin{array}{l}\text { Push return the iterator pointing to the new } \\
\text { inserted element. } O(1)\end{array}$ \\
\hline void push_anywhere_async(const T\&_val) & $\begin{array}{l}\text { Push val on to the last local bContainer } \\
\text { with no return value. } O(1)\end{array}$ \\
\hline
\end{tabular}

\section{C. pList Design and Implementation}

In this section, we describe the pList modules used for storage and data distribution information.

bContainer: For the STAPL pList, we use the STL list as the container of the bContainer. Most pList methods will ultimately be executed on the bContainer using the bContainer's corresponding methods. For example, pList insert will ultimately invoke the STL list insert method. The pList bContainer can also be provided by the user so long as insertions and deletions never invalidate iterators, and the bContainer provides the required domain interface (see below). Additional 
requirements are relative to the expected performance of the methods (e.g., insertions and deletions should be constant time operations).

The pList has a global view of all of the bContainers and knows the order between them in order to provide a unique traversal of all its data. For this reason each bContainer is identified by a globally unique BCID. For static or less dynamic - in terms of number of bContainers - pContainers such as pArray or associative containers, the BCID can be a simple integer. The pList, however, needs a BCID that allows for fast dynamic operations. During the splice operation, bContainers from a pList instance need to be integrated efficiently into another pList instance while maintaining the uniqueness of their BCIDs. For these reasons, the BCID for the pList bContainers is currently defined as follows:

typedef std::pair<plist_bcontainer*, location_identifier> CID

Global Identifiers (GID): Performance and uniqueness considerations similar to those of the bContainer identifier, and the list guarantee that iterators are not invalidated when elements are added or deleted, lead us to use the following definition for the pList GID.

typedef std::pair<std: :list<>: : iterator, BCID> gid;

Since the BCID is unique, the GID is unique as well. With the above definition for GID, the pList can uniquely identify each of its elements and access them independent of their physical location.

Domain: The domain interface for the pList is provided by the pList bContainers. The pList domain is a union of all domains corresponding to individual bContainers. The union domain doesn't replicate any data from the pList but it stores pointers to its bContainers. 


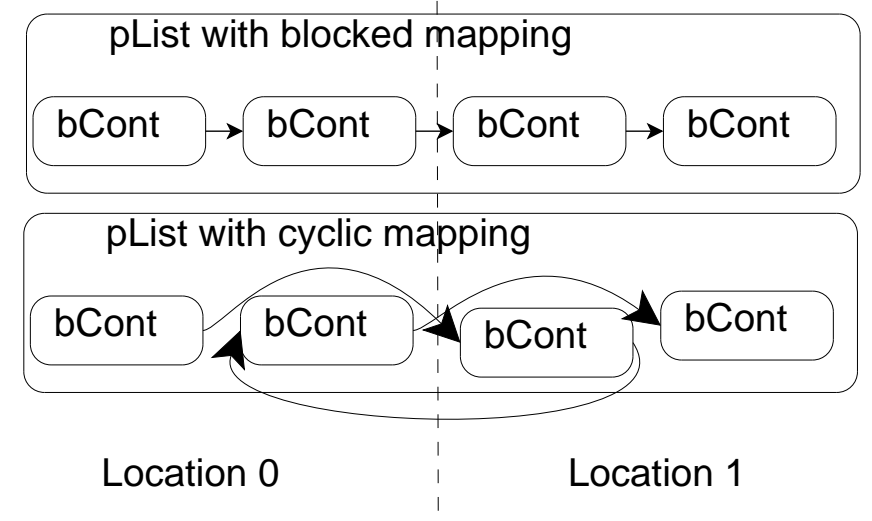

Fig. 37. Different partitions and mappings for pList.

Data Distribution: The data distribution manager for a pList uses a partition and a partition-mapper to describe how the data will be grouped and mapped on locations. The pList specializes the partition mapper to take advantage of the fact that the location identifier is embedded in the bContainer identifier.

The pList uses a dynamic partition that can maintain an arbitrary number of bContainers and elements per location. The partition constructor can take an optional argument, which is the number of desired bContainers and it will allocate them balanced across locations. The allocation can be done in a blocked fashion or in a cyclic fashion as depicted in Figure 37. Subsequent insert and delete operations may lead to imbalanced distributions of elements in the bContainer. The pList provides a method for this situation to redistribute the data so that elements are rebalanced across locations.

pView: The pList currently supports sequence pViews that provide an iterator type and begin() and end() methods. A pView can be partitioned into sub-views. By default the partition of a pList pView matches the subdivision of the list in 
bContainers, thus allowing random access to portions of the pList. This allows parallel algorithms to achieve good scalability as shown in Section D.

pList Container: A typical implementation of a pList method that operates at the element level is included in Figure 38 and uses the invoke skeleton introduced in Chapter IV, Section 6. The run-time cost of the method has three constituents: the time to decide the location and the bContainer where the element will be added (Figure 8, line 5-15), the communication time to get/send the required information (Figure 8, line 10), and the time to perform the operation on a bContainer (Figure 8, line 17).

The complexity of constructing a pList of $N$ elements is $O(M+\log P)$, where $M$ is the maximum number of elements in a location. The $\log P$ term is due to a fence at the end of the constructor to guarantee the pList is in a consistent state. The complexities of the element-wise methods are $O(1)$. Multiple concurrent invocations of such methods may be partially serialized due to concurrent thread-safe accesses to common data. The size and empty methods imply reductions and the complexity is $O(\log P)$, while clear is a broadcast plus the deletion of all elements in each location, so the complexity is $O(M+\log P)$. This analysis relies on the pList bContainer to guarantee that allocation and destruction are linear time operations and size, insert, erase and push_back/front are constant time operations.

The pList also provides methods to rearrange data in bulk. These methods are splice and split to merge lists together and split lists, respectively.

The signature of the pList splice method is:

void pList: splice(iter pos, pList\& pl [, iter it1, iter it2]);

where iter stands for an iterator type, pos is an iterator of the calling pList, pl is another pList, and the optional iterators it1 and it2 are iterators pointing to 


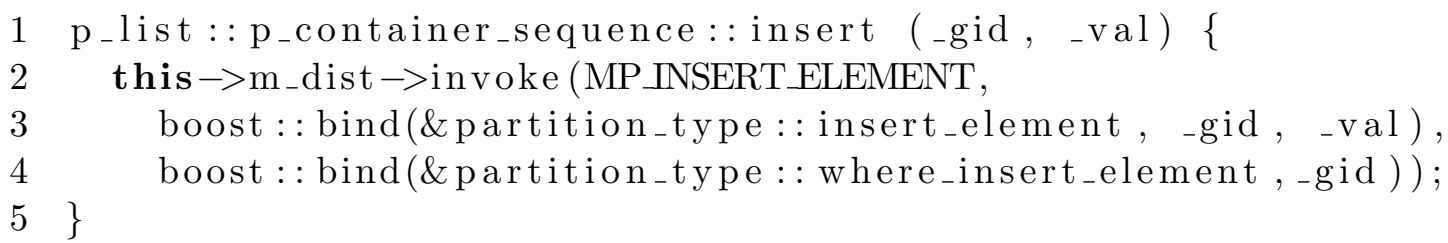

Fig. 38. pList method implementation.

elements of $\mathrm{pl}$. splice removes from $\mathrm{pl}$ the portion enclosed by it1 and it2 and inserts it at pos. By default it1 denotes the begin of $\mathrm{pl}$ and it2 the end.

The complexity of splice depends on the number of bContainers included within it1 and it2. If it1 or it2 points to elements between bContainers, then new bContainers are generated in constant time using sequential list splice. Since the global begin and global end of the pList are replicated across locations, the operation requires a broadcast if either of them is modified.

split is also a member method of pList that splits one pList into two. It is a parallel method that is implemented based on splice with the following signature:

void pList::split(iterator pos, pList\& other_plist)

When pList.split(pos, other_plist) is invoked, the part of pList starting at pos and ending at pList.end() is appended at the end of the other_plist. The complexity of split is analogous to the complexity of splice.

\section{Performance Evaluation}

In this section, we evaluate the scalability of the pList methods. We compare pList and pVector performance, evaluate generic pAlgorithms (p_generate and p_partial_sum) on pList, pArray and pVector, and evaluate an Euler tour imple- 


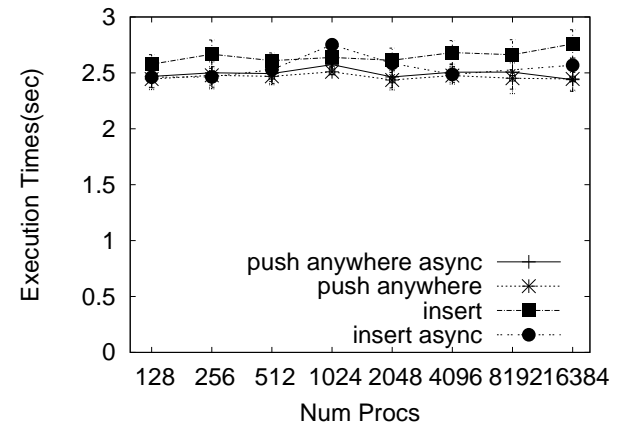

(a) CRAY4: Local Methods

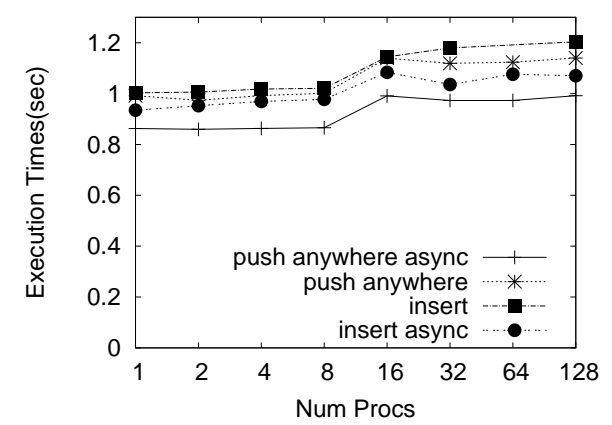

(c) P5-CLuster: Local Methods

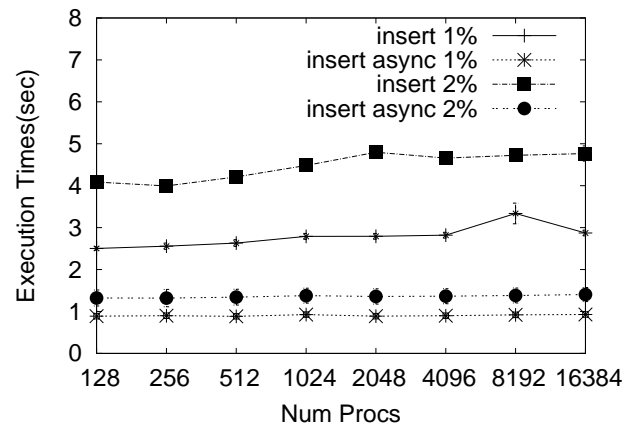

(b) CRAY4: Insert Remote

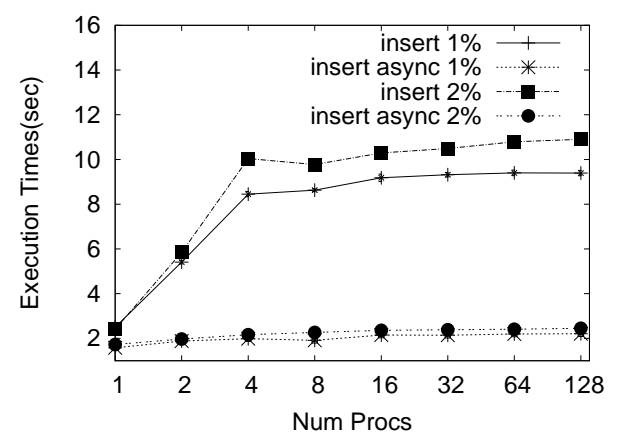

(d) P5-CLuster: Insert Remote

Fig. 39. Execution times for pList methods.

mentation using pList.

E. pList Method Evaluation

Figure 39 shows the execution time of different pList methods. In a first study, all methods are executed locally and we observe in Figure 39(a) that both synchronous and asynchronous methods exhibit scalable performance as they do not incur any communication. In Figure 39(b) we show the execution time for a mix of local and remote method invocations to highlight the advantages of the asynchronous methods over the synchronous methods. In this situation, the synchronous methods incur a 


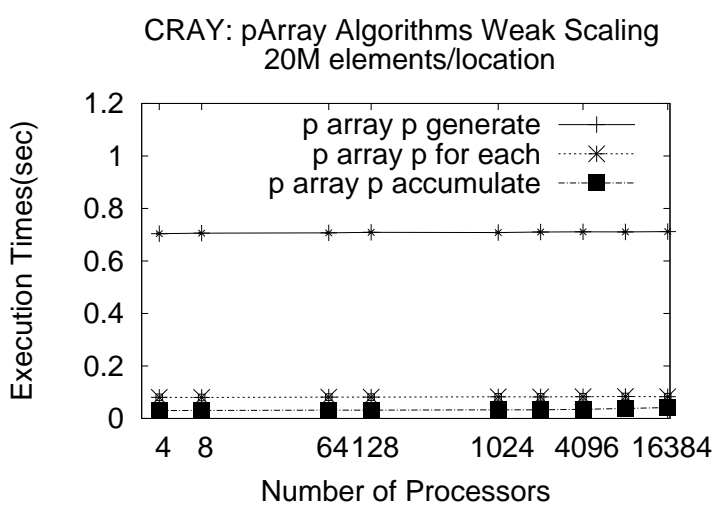

(a) pArray; 20M/Proc

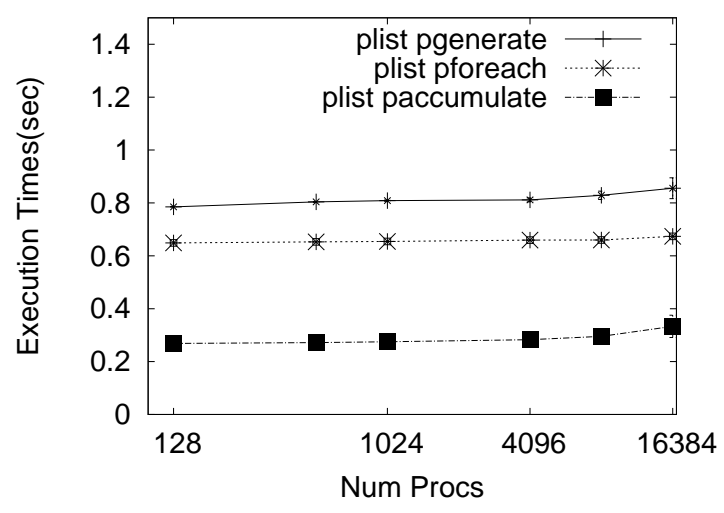

(b) pList; 20M/Proc

Fig. 40. Execution times for p_for_each, p_generate, p_accumulate algorithms on CRAY4 for pArray and pList.

performance overhead being 2.5 times slower. In Figure 39(c) and 39 (d) we show the same experiment on P5-CLUSTER. We observe the same trends as on CRAY4 except that now the difference between synchronous and asynchronous is much larger with the synchronous operations being 5 times slower when using 128 processors.

\section{F. pAlgorithm Comparison}

Figure 40(a) presents the execution times for the p_generate, p_for_each and p_accumulate algorithms on the data of a pArray and a pList on CRAY4 using from 128 to 16384 processors. Figure 40(b) shows the results for the three algorithms on the pList. We observe that the execution time for pList is higher than for pArray, which is the result of the longer access time for the elements of the STL list with respect to the STL valarray. When using 16384 processors there is a $18 \%$ increase in execution time for pArray and a $5 \%$ increase for pList. 


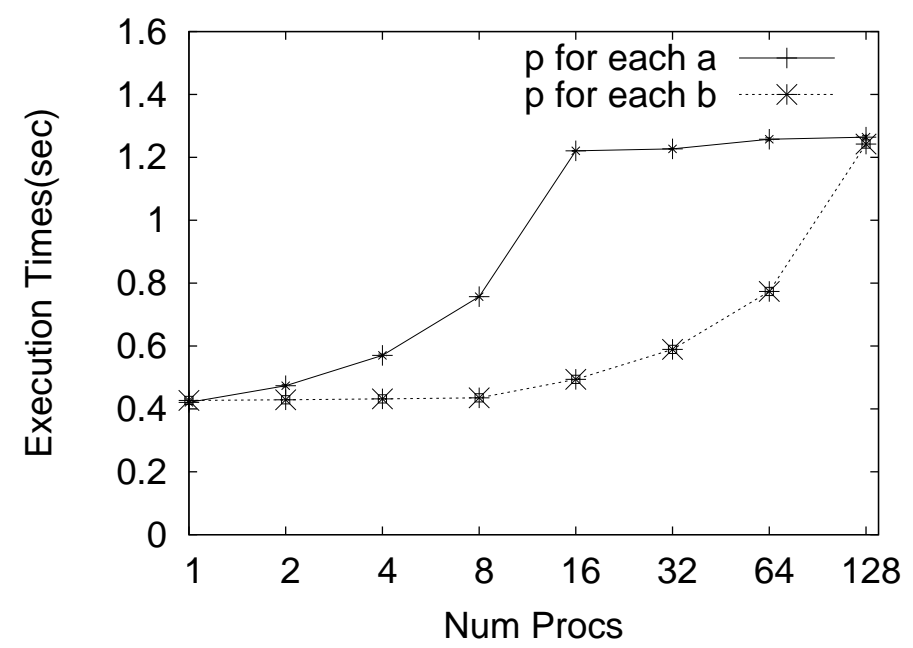

Fig. 41. P5-CLUSTER: Weak scaling for p_for_each allocating processes on the same nodes when possible (curve a) or in different nodes (curve b). Experiments are for 20 million elements/processor.

Figure 41 shows two weak scaling experiments on P5-CLUSTER for two different processor allocation strategies. Each node of P5-CLUSTER has 16 processors. In the figure, p_for_each-a represents the case where all processors are allocated on a single node (possible for 1-16 processors). p_for_each-b represents the case where we use cyclic allocation across 128 processors, e.g., 16 processors would be allocated one per node, and in general, there will be $P / 8$ processors allocated on each node for $P<128$. The reason why the two curves do not match is related to memory bandwidth saturation within a node. In p_for_each-b, the nodes are fully utilized only when running on 128 processors, while for p_for_each-a we use all processors in a node when running on 16 processors or more. These experiments emphasize the importance of a good task placement policy on the physical processors. 


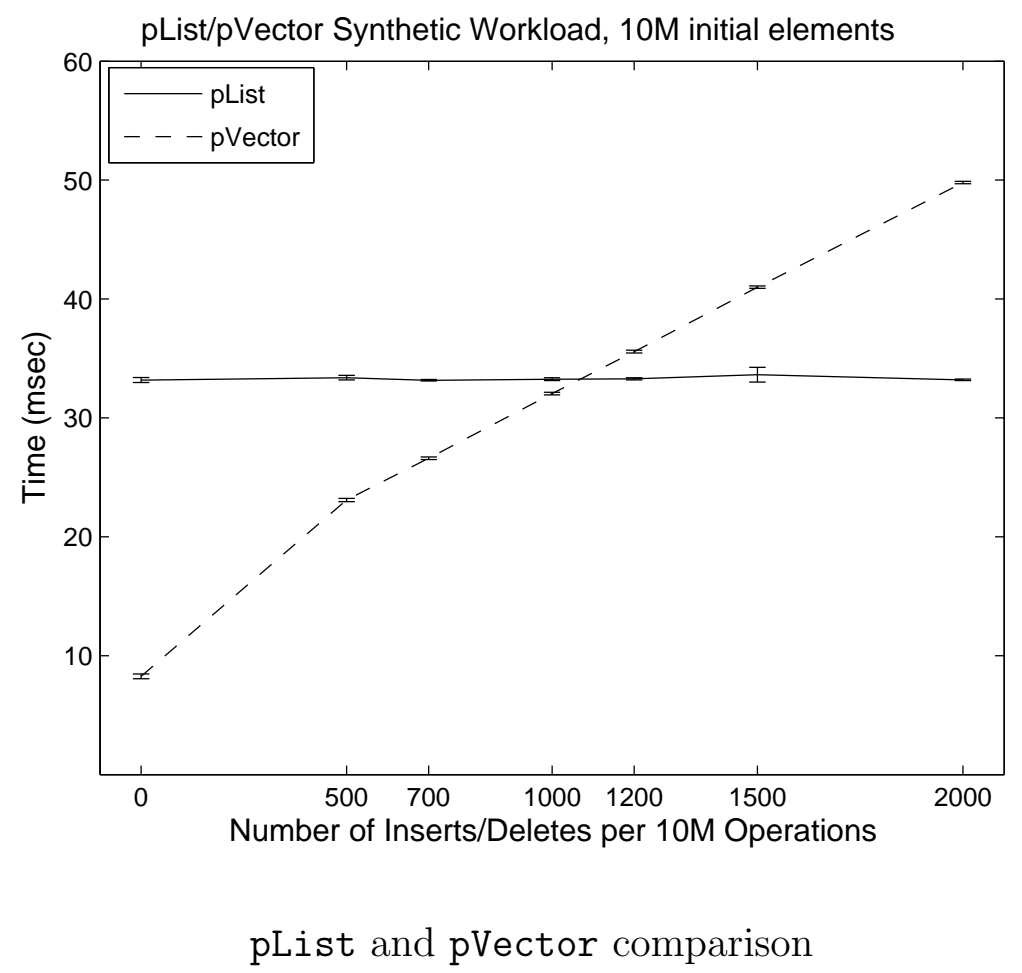

Fig. 42. Comparison pList and pVector dynamic data structures using a mix of 10M operations ( read/write/insert/delete).

G. Comparison of Dynamic Data Structures in STAPL

In this section, we compare the performance of the pList and pVector for various mixes of container operations (i.e., $\operatorname{read}()$, write (), insert () and delete ()$)$. We show that the proportion of operations that modify the container size has substantial effects on runtime, demonstrating the utility of each and that care must be taken in selecting the appropriate parallel data structure.

In Figure 42, we show results for both containers on the P5-CLUSTER for 16 processors and 10 million elements. We perform 10 million operations per container. Each operation is either a read or write of the next element in the container, an 
insertion at the current location, or deletion of the current element. These operations are distributed evenly among the processors, which perform them in parallel. For these experiments, the combined number of insertions and deletions is varied from 0 to 2000, with the remaining operations being an equal number of reads and writes. More insertions or deletions than this cause the runtime of the pVector to increase dramatically.

As expected, the runtime of the pList remains relatively unchanged regardless of the number insertions or deletions, as both operations execute in constant time. The performance of the pVector is better than pList when there are no insertions or deletions. However, at 1200 insertions/deletions, the heavy cost of the operations (all subsequent elements must be shifted accordingly) causes the performance of the two containers to crossover with the pList taking the lead. This experiment clearly justifies the use of the pList in spite of not being a truly random access container like the pVector.

\section{H. Application using pList: Euler Tour}

An Euler Tour (ET) is an important representation of a graph for parallel processing. In particular, the ET, which traverses every edge of the graph exactly once, corresponds to an edge traversal of the graph. Since the ET represents a depth-first-search traversal, when it is applied to a tree it can be used to compute a number of tree functions such as rooting a tree (given a vertex to be root, compute the parent of each vertex in the tree), postorder numbering (compute the postorder number for each vertex in the tree), computing the vertex level (the level of each vertex in the tree), and computing the number of descendants (the number of descendants for each vertex in the tree) [38]. In this application, we convert a tree $T=(V, E)$ into a 
directed tree $T^{\prime}=\left(V, E^{\prime}\right)$ where each edge $(u, v) \in E$ is replaced by two edges $(u, v)$ and $(v, u) . T^{\prime}$ is an Eulerian graph.

The parallel Euler Tour algorithm [38] implemented in STAPL computes an ET of a tree stored in a STAPL pGraph and stores it in a pList. The algorithm executes in parallel traversals on the pGraph pView generating Euler Tour segments that are stored in a temporary pList. Then, the segments are linked together to form the final pList containing the Euler Tour.

The tree ET applications are computed by first using the ET algorithm to compute the ET, and then applying a generic ET algorithm. The generic algorithm first initializes each edge in the tour with a corresponding weight, and then compute prefix sums using the partial sum algorithm. The partial sum result for each edge is copied back to the graph, and the final step is to compute the desired result, e.g., parent, postorder number, vertex level or descendants, using an appropriate function on the prefix sum values.

The performance of the algorithm for computing ET is evaluated by performing a weak scaling experiment on CRAY4 using as input a tree distributed across all locations. The tree is generated by first building a specified number of binary trees in each location and then linking the roots of these trees in a binary tree fashion. The number of remote edges is at most six times of the number of subtrees for each location (for each root of the subtree, one to its root and two to its children in each location, with directed edges for both directions). Figure 43(a) and (b) shows the execution time on CRAY4 for different sizes of the tree and different number of subtrees. The running time increases with the number of vertices per location because the number of edges in the computed ET increases correspondingly. When there are more subtrees specified in each location, there is more communication time taken to link them.

The performance of the ET technique generic algorithms is shown in Figure 44(a), 
CRAY4: Euler Tour Weak Scaling tree: $500 \mathrm{k}$ or $1 \mathrm{M}$ vertices with 1 or 50 subtrees per proc

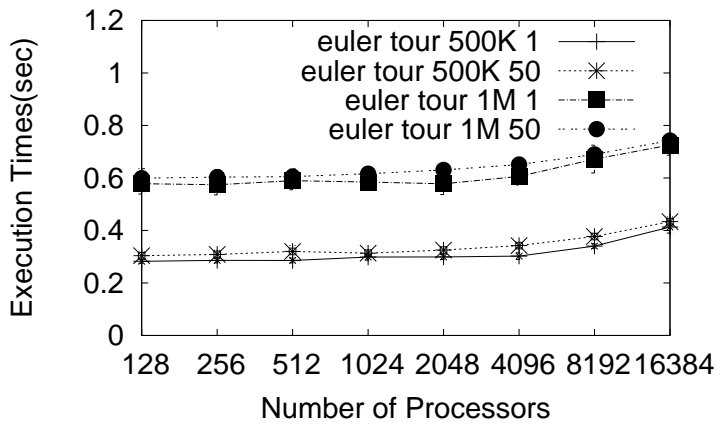

(a) List Graph
CRAY4: Euler Tour Weak Scaling list: $1 \mathrm{M}$ or $2 \mathrm{M}$ vertices with 1 or 1000 lists per proc

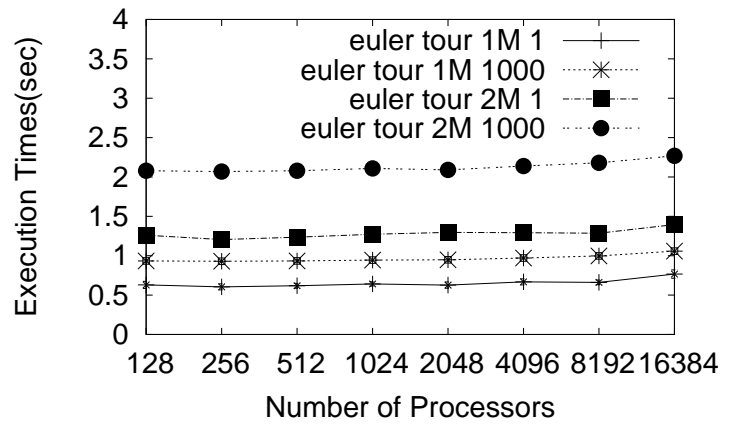

(b) Tree Graph

Fig. 43. Weak scaling of Euler Tour algorithm. Tree made by a single binary tree with $500 \mathrm{k}$ or $1 \mathrm{M}$ subtrees per processor.

(b), (c) and (d) for rooting a tree, computing the postorder numbering, the vertex level, and the number of descendants, respectively. The running time increases with the number of vertices per location because the number of edges increases, which are proportional to the computation. The more subtrees specified in each location, the more segments formed in the pList and the more communication time taken for the partial sum algorithm. 
CRAY4: Rooting Tree Weak Scaling tree: $500 \mathrm{k}$ or $1 \mathrm{M}$ vertices with 1 or 50 subtrees per proc

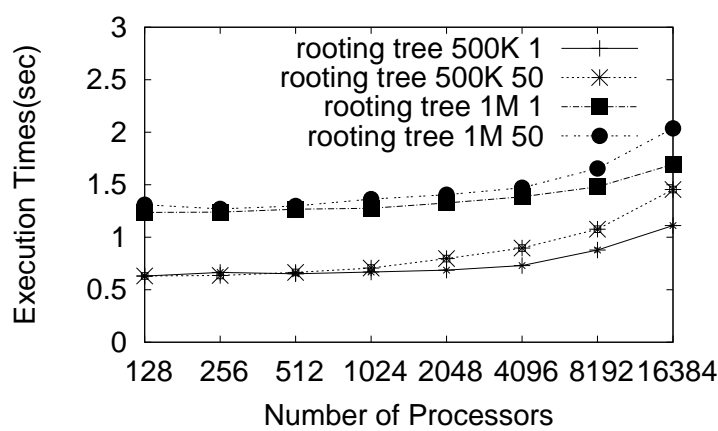

(a) Rooting Tree

CRAY4: Vertex Level Weak Scaling tree: $500 \mathrm{k}$ or $1 \mathrm{M}$ vertices with 1 or 50 subtrees per proc

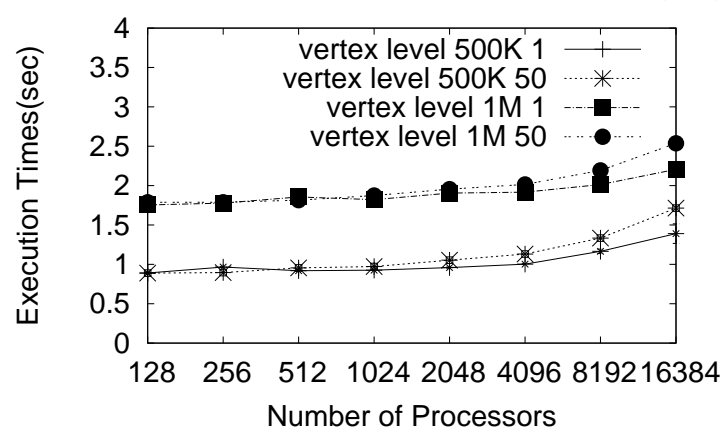

(c) Computing Vertex Level
CRAY4: Postorder Numbering Weak Scaling tree: $500 \mathrm{k}$ or $1 \mathrm{M}$ vertices with 1 or 50 subtrees per proc

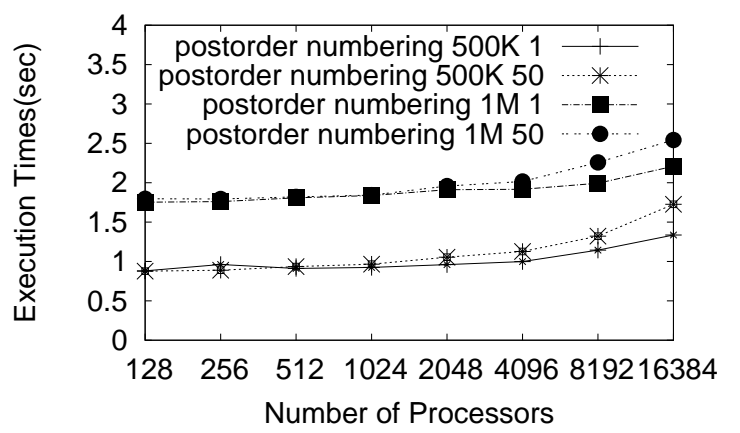

(b) Postorder Numbering

CRAY4: Number of Descendants Weak Scaling tree: $500 \mathrm{k}$ or $1 \mathrm{M}$ vertices with 1 or 50 subtrees per proc

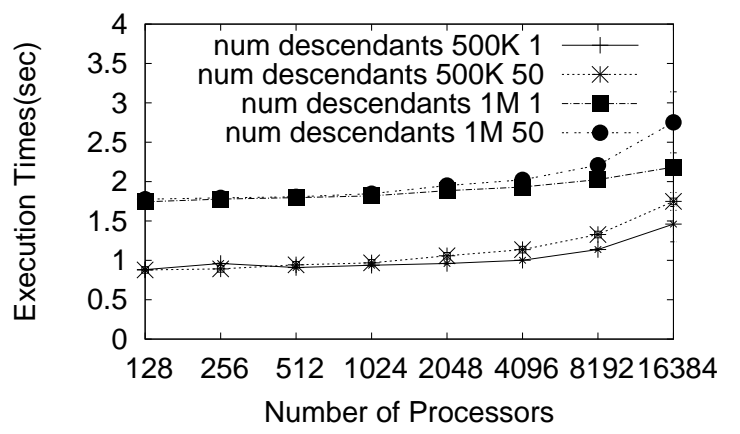

(d) Computing Number of Descendants

Fig. 44. Execution times for Euler Tour and its applications using a tree made by a single binary tree with $500 \mathrm{k}$ or $1 \mathrm{M}$ subtrees per processor. 


\section{CHAPTER XI}

\section{THE STAPL PGRAPH}

The STAPL pGraph is a generic data structures that consists of a collection of vertices and relations between vertices called edges. We use the following notation for a graph: $G=(V, E)$. The pGraph associates a vertex property with each vertex and an edge property with each edge. These are template arguments that are passed by the user when instantiating a pGraph. Additionally, using template arguments, users can indicate if the graph is directed (directedness) and if the graph allows multiple edges between same source and destination (multiplicity). Similar to other STAPL pContainers, users can specify an optional partition and traits. Thus, the pGraph declaration is the following:

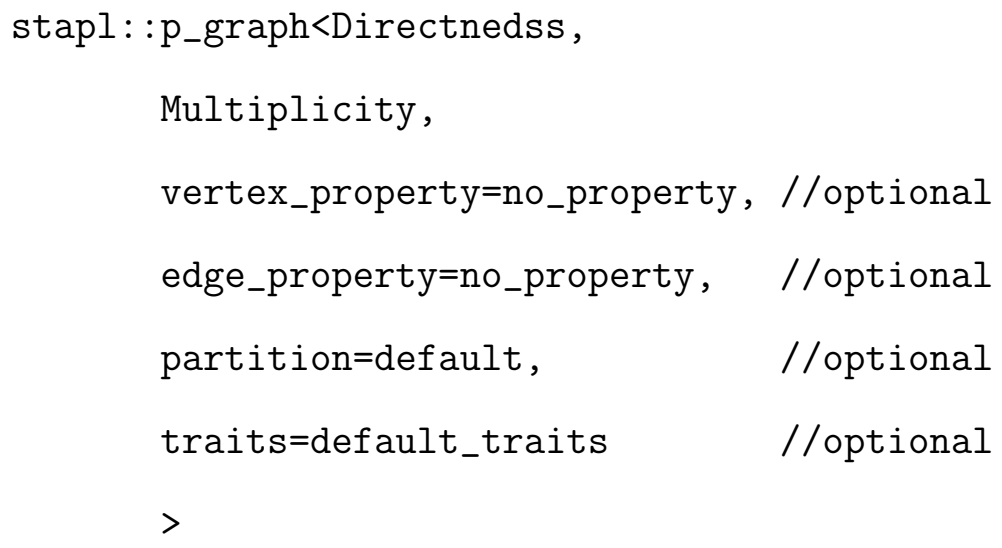

If the user application doesn't require vertex or edge properties, then the special value stapl: :no_property can be used. The traits allow users to customize low level details such as the storage for vertices and edges. Before we introduce the pGraph user interface, we define a set of concepts in the next section that are required to properly describe the methods. 
A. pGraph Example

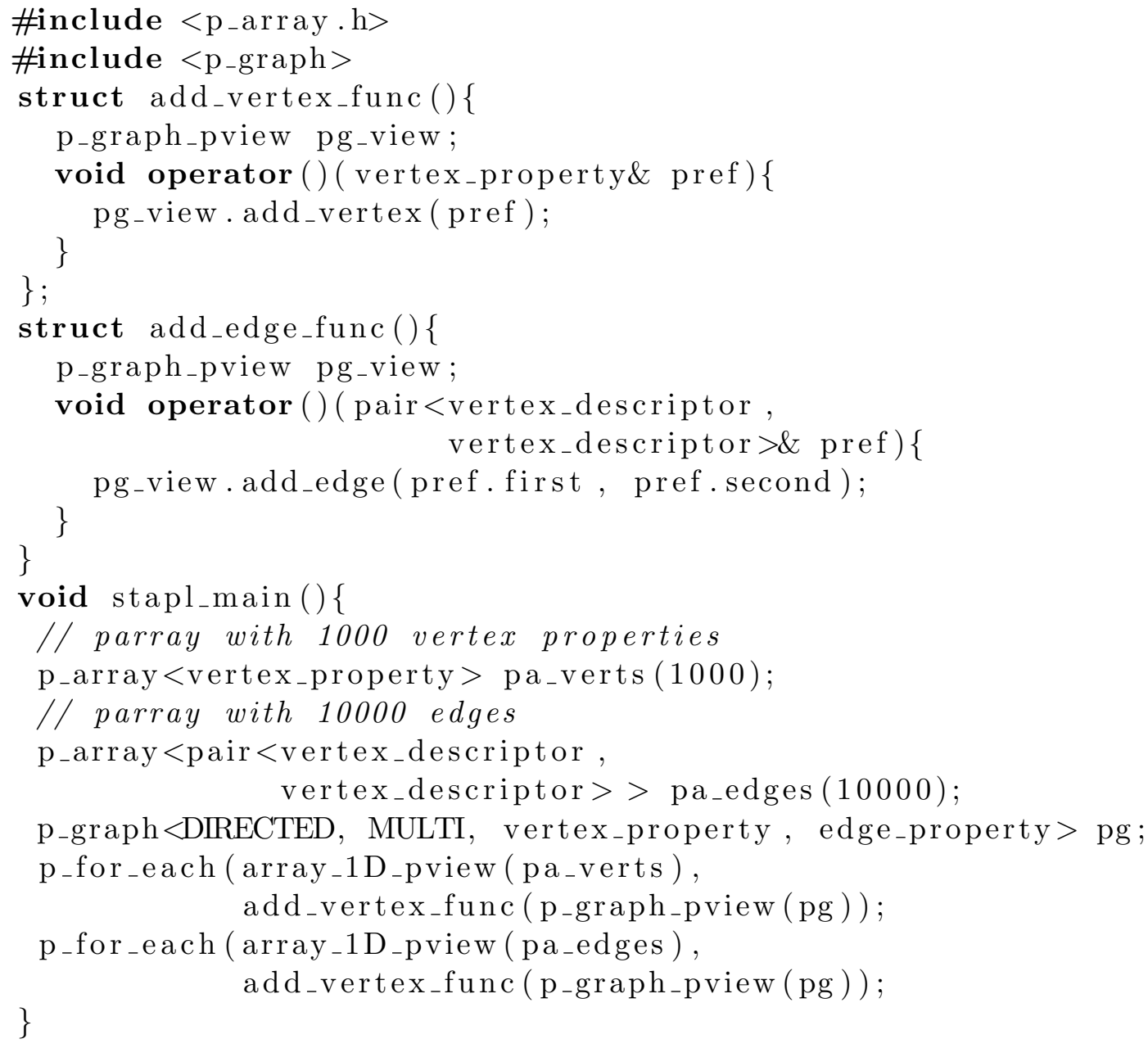

Fig. 45. pGraph example.

In Figure 45 we include a simple example using the pGraph data structure. At line 17 and 19 we declare a pArray of vertex properties and a pArray of edges, respectively. 
Subsequently we invoke two p_for_each invocations with functors to create vertices and edges for all elements in the input pArrays. The functors call add_vertex and add_edge methods of the pGraph pView which recursively invoke the add_vertex method of the pGraph.

B. pGraph Concepts and Interfaces

When implementing or using STAPL graphs the user needs to be aware of a number of additional concepts relative to the pArray and pList. The pGraph concepts and their associated properties are introduced in this section.

Vertex Descriptor: The vertex descriptor uniquely identifies a vertex. The vertex descriptor has to be default constructable, assignable and equality comparable. Adding edges or finding vertices will be based on vertex descriptors.

Edge Descriptor: The edge descriptor uniquely identifies an edge. It provides methods to return the source and target vertex and a unique edge identifier. The reason the edge identifier is needed is to distinguish between vertices with the same source and destination.

Vertex Iterator: The vertex iterator is an overloaded iterator concept. It implements the STL bidirectional access iterator concept (operator,++-- ) and can be dereferenced to provide a Vertex Reference.

Vertex Reference: We provide two vertex reference concepts. They correspond to the case where the graph does not have properties associated with the vertex (vertex_reference $<$ no_property $>$ ) and the case where the graph has properties associated with a vertex (vertex_reference $<$ vertex property $>$ ). The vertex reference is obtained by dereferencing a vertex iterator. It provides the interface in Table XXV. 
Table XXV.: Vertex reference interface. Property related interface is only for vertex_reference $<$ vertex property $>$.

\begin{tabular}{|l|l|}
\hline Defined Type & Description \\
\hline vertex_descriptor & Vertex descriptor type \\
\hline property_type & $\begin{array}{l}\text { Property type for graphs with no property is a special } \\
\text { type no_property }\end{array}$ \\
\hline adj_edge_iterator & $\begin{array}{l}\text { Adjacency edge iterator type. This type of iterator } \\
\text { allows to iterate over the adjacent edges of a vertex } \\
\text { referred by the current iterator }\end{array}$ \\
\hline Method & Description \\
\hline adj_edge_iterator begin() & $\begin{array}{l}\text { Returns an adjacency edge iterator pointing to a first } \\
\text { outgoing edge. } O(1)\end{array}$ \\
\hline adj_edge_iterator end () & $\begin{array}{l}\text { Returns an adjacency edge iterator pointing to a last } \\
\text { outgoing edge. } O(1)\end{array}$ \\
\hline view edges() & Returns a pView over the adjacent edges. $O(1)$ \\
\hline size_t size() const & Returns the number of outgoing edges. $O(1)$ \\
\hline Property\& property() & Returns the property associated with the vertex. $O(1)$ \\
\hline
\end{tabular}

Edge Iterator: Similar to the vertex iterators, the graph provides edge iterators. First, the adj_edge_iterator iterates over the adjacency list of a vertex and secondly, edge_iterator iterates over all edges of the graph. It can be dereferenced to provide an Edge Reference.

Edge Reference: The edge reference is obtained by dereferencing an edge iterator or an adjacent edge iterator. It provides the interface in Table XXVI. 
Table XXVI.: Edge reference interface.

\begin{tabular}{|l|l|}
\hline Defined Type & Description \\
\hline property_type & $\begin{array}{l}\text { Property is of type no_property meaning the edges are } \\
\text { without properties }\end{array}$ \\
\hline vertex_descriptor & Vertex descriptor type \\
\hline $\begin{array}{l}\text { vertex_descriptor source }() \\
\text { const }\end{array}$ & Returns vertex descriptor for the source. $O(1)$ \\
\hline $\begin{array}{l}\text { vertex_descriptor target() } \\
\text { const }\end{array}$ & Returns vertex descriptor for the target. $O(1)$ \\
\hline size_t id() const & Returns the unique id of the edge. $O(1)$ \\
\hline Property\& property() & Returns the property associated with the edge. $O(1)$ \\
\hline
\end{tabular}

\section{C. pGraph Class Hierarchy}

The STAPL pGraph provides the following classes of graphs: incidence_p_graph, static_p_graph, dynamic_p_graph, directed_p_graph, undirected_p_graph, multiedges_p_graph and non_multi_edges_p_graph. Each of these specify an interface and corresponding properties as summarized in Figure 46(a). More details about the functionality of individual methods are included in Table XXVII.

Corresponding to each of the graph concepts in Figure 46(a) there is a pGraph pView interface. Generic parallel graph algorithms need to specify the requirements for the pView that they can operate on. For example certain algorithms may require an incidence_p_graph_view that models the incidence_p_graph concept.

STAPL provides a default implementation of the pGraph concepts from Figure 46(a) as depicted in Figure 46(b). There is a p_container_relation class derived from p_container_dynamic class of the framework that implements the interfaces corresponding to incidence, static and dynamic graph concepts. Directed pGraph specifies additional methods such as get_out_degree(). Undirected guarantees that 


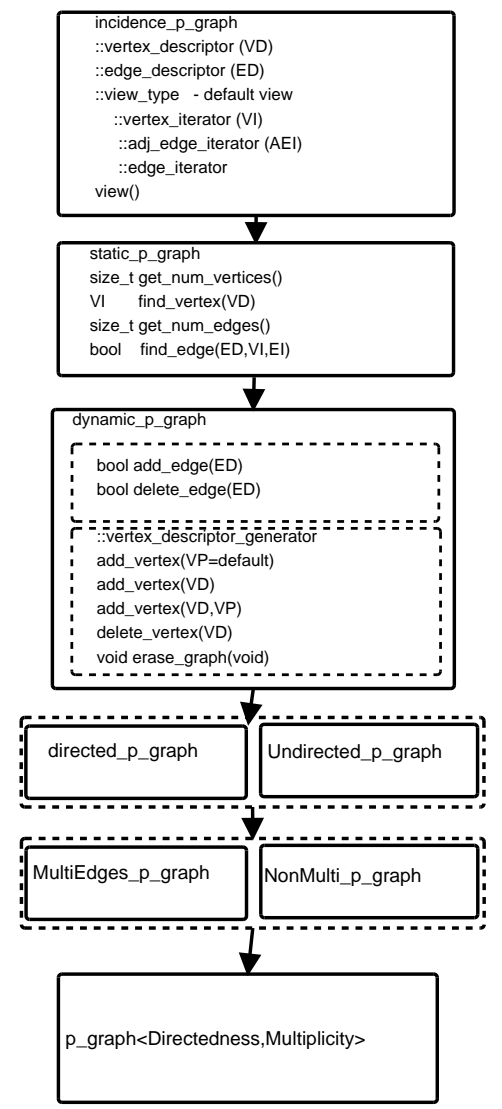

(a)

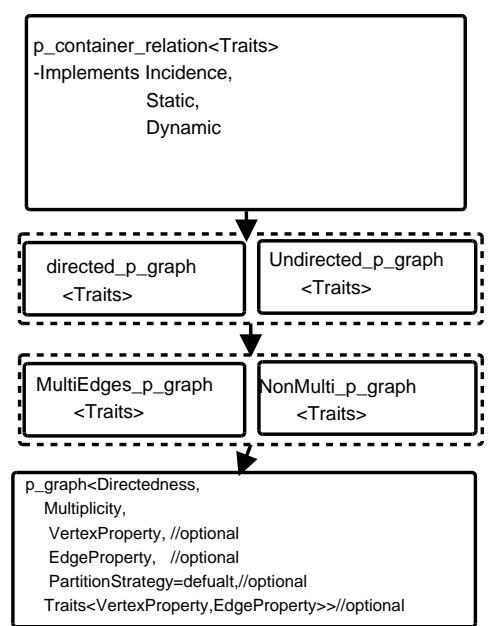

(b)

Fig. 46. Graph hierarchy of concepts (a) and hierarchy design for STAPL implementation of graph (b). AEI is an iterator over adjacent edges only; EI iterates over all edges of a graph and it is an extension of the adjacency edge iterator(AEI).

for every edge $(u, v)$ there is an edge $(v, u)$. Additionally, if the graph has properties the property is shared by the two edges. The non multiple edges class specializes the add_edge method to guarantee that there are no edge duplicates in the pGraph. The pGraph class at the bottom of the hierarchy in Figure 46(b) uses the template arguments to select the proper derivation chain and other internals such as partition and storage. 
Table XXVII.: pGraph interface.

\begin{tabular}{|c|c|}
\hline Define Type & Description \\
\hline vertex_property & $\begin{array}{l}\text { property stored on vertices; it can be of } \\
\text { no_property type }\end{array}$ \\
\hline edge_property & $\begin{array}{l}\text { property stored on edges; it can be of } \\
\text { no_property type }\end{array}$ \\
\hline vertex_descriptor & vertex descriptor type \\
\hline edge_descriptor & edge descriptor type \\
\hline vertex_reference & vertex reference type \\
\hline edge_reference & edge reference type \\
\hline vertex_descriptor add_vertex() & $\begin{array}{l}\text { Add a vertex with default allocated prop- } \\
\text { erty. } O(1)\end{array}$ \\
\hline $\begin{array}{l}\text { vertex_descriptor add_vertex(const ver- } \\
\text { tex_property\& pref) }\end{array}$ & $\begin{array}{l}\text { Add a vertex with the specified property. } \\
O(1)\end{array}$ \\
\hline $\begin{array}{lll}\text { vertex_descriptor } & \text { add_vertex(const ver- } \\
\text { tex_descriptor vd) } & & \end{array}$ & $\begin{array}{l}\text { Add a vertex with the specified descriptor. } \\
O(1)\end{array}$ \\
\hline $\begin{array}{l}\text { vertex_descriptor add_vertex(const ver- } \\
\text { tex_descriptor vd, const vertex_property\& } \\
\text { pref) }\end{array}$ & $\begin{array}{l}\text { Add a vertex with the specified descriptor } \\
\text { and property. } O(1)\end{array}$ \\
\hline $\begin{array}{l}\text { bool delete_vertex(const vertex_descriptor } \\
\text { vd) }\end{array}$ & $\begin{array}{l}\text { Delete the vertex with the specified descrip- } \\
\text { tor. } O(N V \text { erts })\end{array}$ \\
\hline $\begin{array}{l}\text { edge_descriptor } \\
\text { edge_descriptor ed) }\end{array}$ & $\begin{array}{l}\text { Add an edge with the given descriptor spec- } \\
\text { ifying the source and target vertices. } O(1)\end{array}$ \\
\hline $\begin{array}{l}\text { edge_descriptor } \\
\text { edge_descriptor ed, const } \\
\text { pref) }\end{array}$ & $\begin{array}{l}\text { Add an edge with the specified descriptor } \\
\text { and property. } O(1)\end{array}$ \\
\hline $\begin{array}{l}\text { void add_edge_async(const edge_descriptor } \\
\text { ed) }\end{array}$ & $\begin{array}{l}\text { Asynchronously add an edge with the given } \\
\text { descriptor specifying the source and target } \\
\text { vertices; Faster than the synchronous vari- } \\
\text { ant. } O(1)\end{array}$ \\
\hline $\begin{array}{l}\text { void add_edge_async(const edge_descriptor } \\
\text { ed, const edge_property\& pref) }\end{array}$ & $\begin{array}{l}\text { Asynchronously add an edge with the spec- } \\
\text { ified descriptor and property; Faster than } \\
\text { the synchronous variant. } O(1)\end{array}$ \\
\hline bool delete_edge(const edge_descriptor ed) & $\begin{array}{l}\text { Delete the edge with the specified descrip- } \\
\text { tor. } O(N E d g e s / N V \text { erts }) \text { - proportional } \\
\text { with the number of edges per vertex. }\end{array}$ \\
\hline $\begin{array}{l}\text { vertex_iterator find_vertex(const } \\
\text { tex_descriptor vd) }\end{array}$ & $\begin{array}{l}\text { Find the vertex with the given ver- } \\
\text { tex_descriptor. Returns graph. end () if ver- } \\
\text { tex not found. } O(1)\end{array}$ \\
\hline
\end{tabular}


Table XXVII continued

\begin{tabular}{|l|l|}
\hline Method & Description \\
\hline $\begin{array}{l}\text { edge_iterator find_edge(const } \\
\text { edge_descriptor ed, vertex_iterator* vi, } \\
\text { edge_iterator* ei) }\end{array}$ & $\begin{array}{l}\text { Find the edge with the given } \\
\text { edge_descriptor. Returns graph.end() } \\
\text { if vertex not found. Also returns the } \\
\text { vertex iterator of the source vertex, and } \\
\text { the corresponding adjacency edge iterator. } \\
O(N \text { edges } / N V \text { erts })\end{array}$ \\
\hline size_t get_num_vertices () & $\begin{array}{l}\text { Returns the number of vertices in the graph. } \\
O(\log (P))\end{array}$ \\
\hline size_t get_num_edges () & $\begin{array}{l}\text { Returns the number of edges in the graph. } \\
O(N V \text { erts } / P+\log (P))\end{array}$ \\
\hline void clear () & $\begin{array}{l}\text { Clears the graph, erasing all edges and ver- } \\
\text { tices. } O(N / P+\log (P))\end{array}$ \\
\hline bool empty () & $\begin{array}{l}\text { Returns if the graph is empty (has no ver- } \\
\text { tices and edges). } O(\log (P))\end{array}$ \\
\hline
\end{tabular}

In comparison with other graph libraries such as PBGL[29], for better encapsulation, we decided to have each concept correspond to a $\mathrm{C}++$ class rather than having a mix of classes and stand alone functions. For example, to add a vertex or an edge in STAPL the user would say graph.add_vertex() and graph.add_edge(source, target), rather than add_vertex (graph) or add_edge (source, target, graph) as is done in PBGL. In addition to these syntactic differences, we now briefly summarize some other important semantic differences between the STAPL pGraph and PBGL that may favor our approach for certain applications. First, the vertex and edge descriptor are uniquely associated with vertices and edges of the graph and will not change during the lifetime of the container. For PBGL, the descriptors are recomputed when a vertex is removed for certain storages leading to possible inconsistencies. Also, the vertex descriptor changes from BGL to PBGL by incorporating knowledge about the location where the vertex lives. In STAPL, we use the same vertex descriptor for 
the sequential and the parallel graph and store location information in the data distribution manager. By not associating location information with the vertex descriptor, we can migrate vertices in our framework without invalidating descriptors to which other locations may have references.

Another important difference between pGraph and PBGL is the fact that pGraph provides a shared memory view to the user allowing threads on one location to access the entire graph in a shared memory fashion.

\section{D. pGraph Design and Implementation}

In this section we describe the pGraph default implementation of the main concepts required by the framework

bContainer: We use the sequential STAPL generic graph implementation for bContainers but other existing graph libraries such as BGL can be easily integrated. Most pContainer methods will ultimately be executed at the bContainer level. For example, pGraph add_vertex will ultimately invoke the sequential add_vertex method in one of the pGraph bContainers.

Global Identifiers (GID): The vertex descriptor is used as the GID and the default pGraph implementation uses an unsigned integer for this. An important property that differentiates the pGraph from other libraries is the fact that the vertex descriptor associated with a vertex remains valid as long as the vertex exists in the pGraph. Other libraries such as PBGL, depending on the storage chosen, rename all vertices in the graph to guarantee that they are in a contiguous range from zero to the number of vertices.

Partition: For pGraph, the partition of the set of vertices and edges into subsets is of crucial importance. The performance of the pGraph algorithms is often directly 
related with the number of edges crossing the different sets. The default partitions provided by the framework partition the set of available vertices. The edges are implicitly partitioned based on their source vertices. We introduce next three partitions that can be used with the pGraph. First, a static balanced partition with a closed form solution mapping GIDs to bContainers. This is similar to the pArray balanced partition introduced in Chapter IV, Section E. While this partition performs fast mappings of vertex descriptors to sub-domains, it can be used only with graphs with a fixed number of vertices that only allow edges to be inserted or deleted.

The framework also provides two dynamic distributed partitions that allow arbitrary mappings of vertices to sub-domains. These two partitions can form the basis of smarter partitions that can integrate external tools such as Metis [42] or Chaco [32] to specify the decomposition (partition) of vertices. They are implemented using a distributed directory where a certain location is responsible for always knowing where a particular element lives and the location that actually stores the element needs to inform the directory with information about the element. When invoking a method on a specific element the partition may not have locally all the information about where the element is located. When this happens the partition has two options: (1) perform necessary communication and retrieve the full information about where the bContainer that owns the element, followed by the invocations of the method on the bContainer or (2) provide to the framework partial information about a new location that may know more information about the given element. In the second situation the pContainer simply forwards the method invocation to the provided location and the lookup procedure is recursively applied there. For asynchronous methods, forwarding the method based on partial information rather than trying to completely perform the address resolution, possibly using synchronous communication, has performance benefits that we analyze in Section F. 


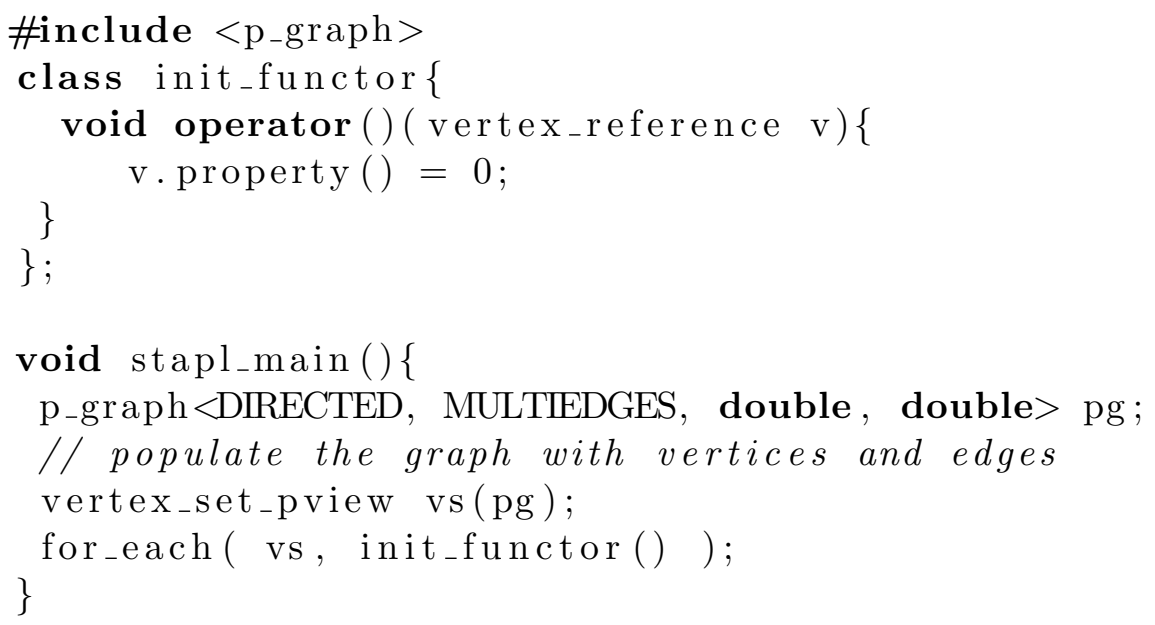

Fig. 47. pGraph pViews example.

Partition Mapper: Similar to other STAPL pContainers the pGraph can use any of the following partition mapper: partition_mapper_generic, partition mapper_blocked, partition_mapper_cyclic or partition_mapper_identity.

\section{E. pGraph pViews}

In Section $\mathrm{C}$ we introduced a taxonomy of various graph concepts. Corresponding to each of the concepts there is a well defined interface and graph algorithms are written in terms of these interfaces (e.g., pViews). These pViews can be defined on top of the STAPL pGraph but they can also be defined on top of other containers or on the composition of containers. For example, an incidence_p_graph_pview can be defined on top of a pArray of vertices where a vertex is a list of edges. In this section, we describe the pViews that can be defined on top of the pGraph data structure.

For simple algorithms that access all vertices or all edges, we define a vertex_set pview and edge_set_pview. As illustrated in Figure 47 these pViews can be passed as 


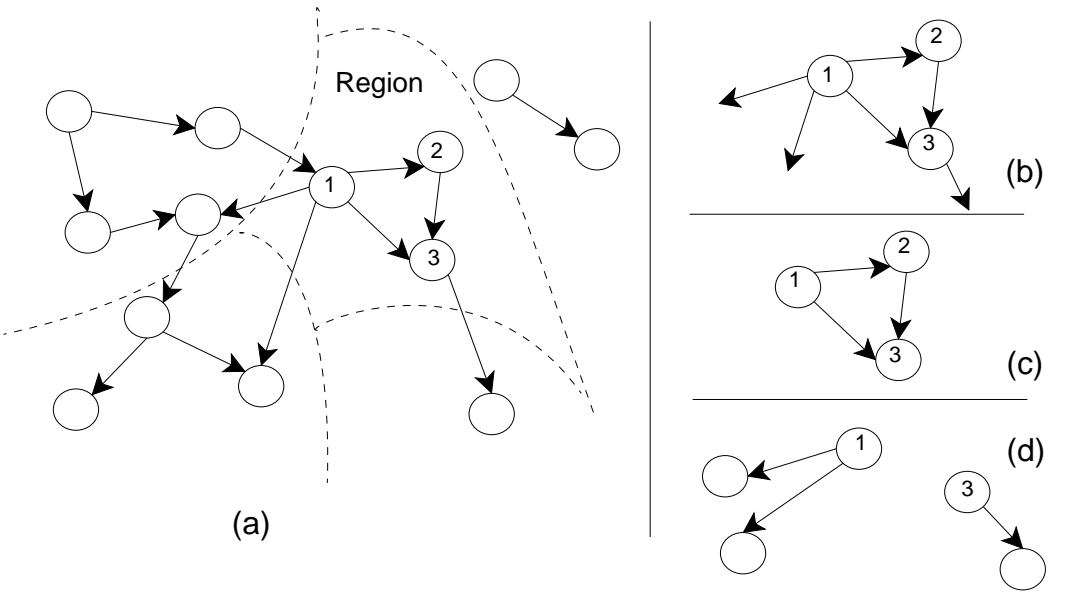

Fig. 48. pGraph pViews: (a) pGraph partitioned pView, (b) region_pview, (c) inner_pview and (d) boundary_pview.

arguments to STL like pAlgorithms. The p_for_each computation is used to initialize all vertex properties to zero.

pGraph Partitioned pView: In Figure 48(a) we depict a possible partition of a pGraph according to the dotted lines. For each of the regions of the decompositions we distinguish two categories of edges: normal edges where source and destination vertex are within the same sub domain and boundary edges where the target of the edge is not within the current region.

The framework provides a partitioned pGraph pView that can be used by various pGraph algorithms. Each of the sub views of the partitioned graph pView provides methods to obtain the following pViews:

- Region pView: (Figure 48(b)) Contains normal and boundary edges; it is not a complete graph. This is an intuitive way of partitioning a pGraph and leads to efficient parallel algorithms.

- Inner pView: (Figure 48(c)) A pView with the boundary edges stripped out. 
This is a complete graph and can be passed to sequential graph algorithms.

- Boundary pView: (Figure 48(d)) An edge pView containing only the boundary edges.

\section{F. Performance Evaluation}

In this section, we evaluate the scalability of the pGraph parallel methods introduced in this chapter, discussing different performance trade-offs. We evaluate several graph pAlgorithms such as Euler tour and different traversals.

\section{1. pGraph Methods Evaluation}

The pGraph is represented as an adjacency list and depending on its properties, different bContainers can be used to optimize the data access. Here, we evaluate a static and a dynamic pGraph. The static pGraph allocates all its vertices in the constructor and subsequently only edges can be added or deleted. It uses a static partition that is implemented as a closed-form solution and has a bContainer that uses a std: : vector to store the vertices and std: :list to store edges. The dynamic pGraph uses a distributed directory to implement its partition and its bContainer uses std: :hash map for vertices and std: :list for edges. We chose the std: :hash_map in the dynamic case because it allows for fast insert and find operations. As described in Chapter V, in Figure 16 the pGraph container is one class and the static versus dynamic behavior is achieved by passing the corresponding template arguments to it.

We include in this section results for a weak scaling experiment on CRAY4 and P5-CLUSTER using a 2D torus where each processor holds a stencil of $1500 \times 1500$ vertices and corresponding edges, a stencil of $15 \times 150000$ and a random graph as specified in the SSCA2 benchmark [7]. SSCA2 generates a set of clusters where each 
CRAY: Static pGraph Methods Weak Scaling SSCA\#2, 500K vertices, $11 \mathrm{M}$ edges/location

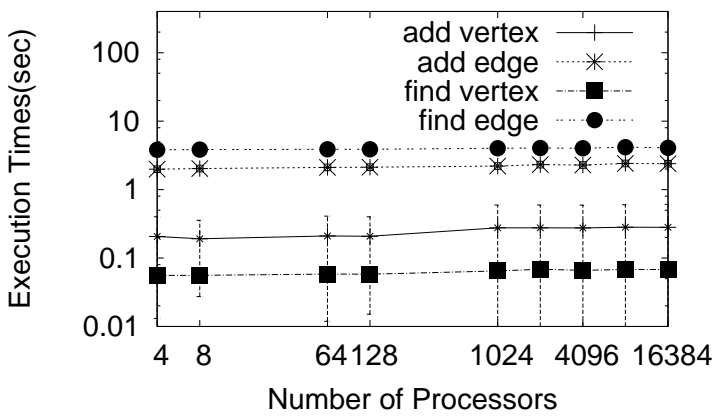

(a) SSCA Static pGraph

CRAY: pGraph Methods Weak Scaling Mesh,2.25M vertices,4.5M edges/location

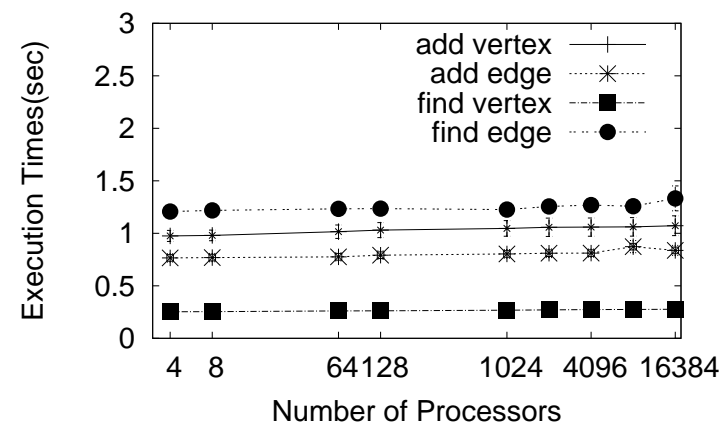

(c) $1500 \times 1500$ Mesh Static

CRAY: pGraph Methods Weak Scaling Mesh,2.25M vertices,4.5M edges/location

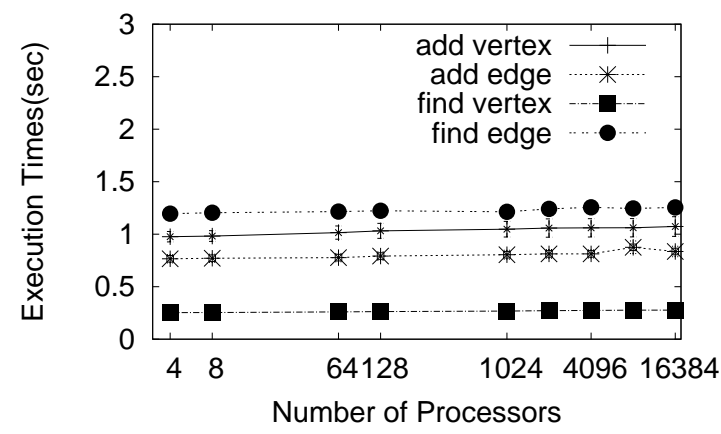

(e) $15 \times 150000$ Mesh Static
CRAY: Dynamic pGraph Methods Weak Scaling

SSCA\#2, 500K vertices, $11 \mathrm{M}$ edges/location

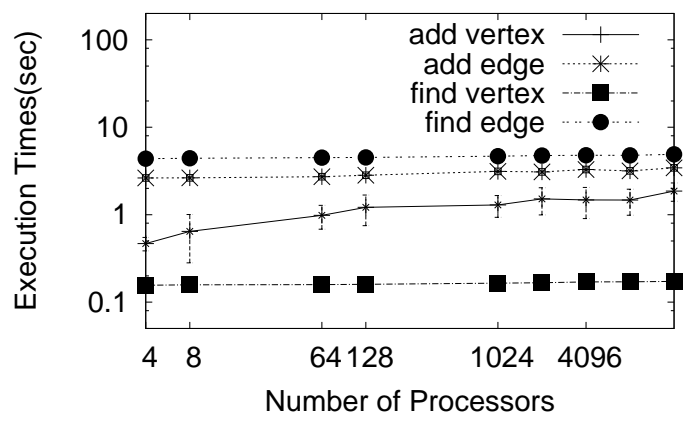

(b) SSCA Dynamic pGraph

CRAY: Dynamic pGraph Methods Weak Scaling Torus, $2.25 \mathrm{M}$ vertices, $4.5 \mathrm{M}$ edges/location

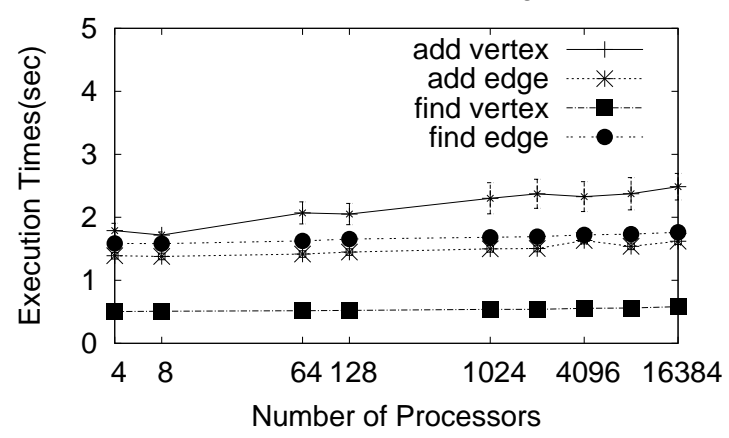

(d) $1500 \times 1500$ Mesh Dynamic

CRAY: Dynamic pGraph Methods Weak Scaling Torus, $2.25 \mathrm{M}$ vertices, $4.5 \mathrm{M}$ edges/location

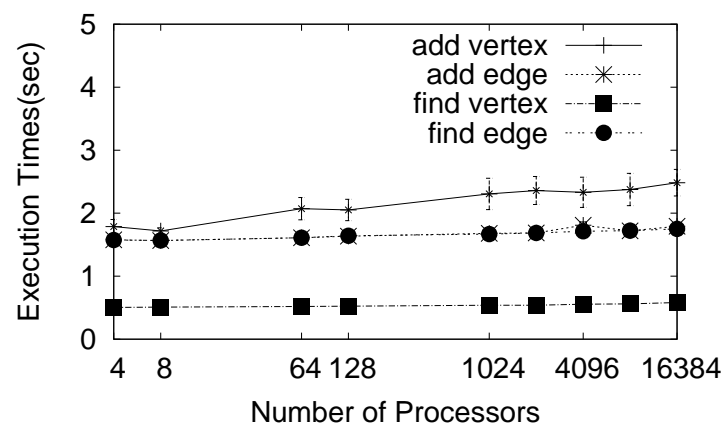

(f) $15 \times 150000$ Mesh Dynamic

Fig. 49. CRAY4: Evaluation of static and dynamic pGraph methods while using the SSCA2 graph generator. The input graph has $500 \mathrm{k}$ vertices, $11.5 \mathrm{M}$ edges, $\sim 40$ remote edges per location, $\sim 23$ edges per vertex. (a) For the static pGraph all vertices are built in the constructor; (b) The dynamic pGraph inserts vertices using add_vertex method. 


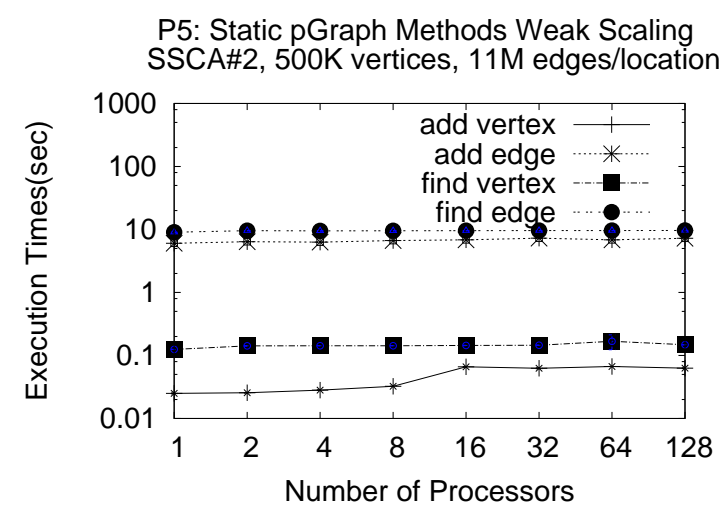

(a) SSCA Static pGraph

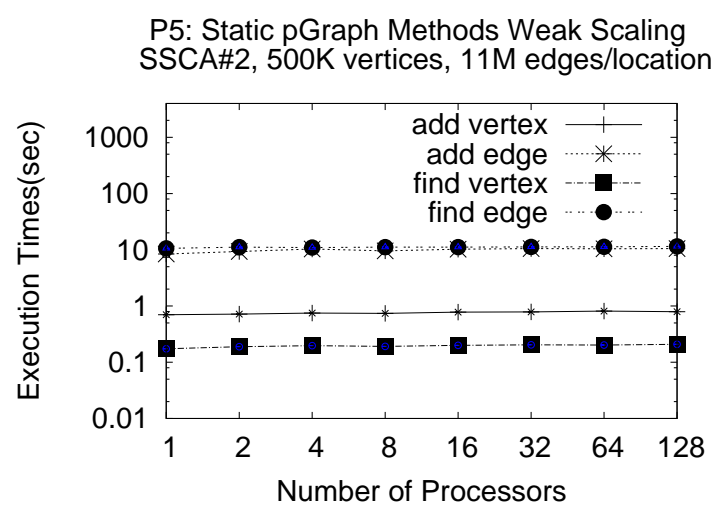

(b) SSCA Dynamic pGraph

Fig. 50. P5-CLUSTER: Evaluation of static and dynamic pGraph methods while using the SSCA2 graph generator. The input graph has 500k vertices, $11.5 \mathrm{M}$ edges, $\sim 40$ remote edges per location, $\sim 23$ edges per vertex. (a) For the static pGraph all vertices are built in the constructor; (b) The dynamic pGraph inserts vertices using add_vertex method.

cluster is densely connected and the inter cluster edges are sparse. We use the following parameters for SSCA2: cluster size $=(V / P)^{1 / 4}$, maximum number of parallel edges is 3, maximum edge weight is $V$, probability of intra clique edges is 0.5 and probability of an edge to be unidirectional 0.3 . Figure 49 shows the execution time for add_vertex, add_edge, find_vertex and find_edges on CRAY4 and Figure 50 for P5-CLUSTER. We include results for a static pGraph where vertices are allocated in the constructor and a dynamic pGraph where the container is initially empty and vertices are added using add_vertex. As seen in the plots, the methods scale well up to 24000 processors on CRAY4 and up to 128 on the P5-CLuster. The addition of edges is a fully asynchronous parallel operation. Adding vertices in the dynamic pGraph causes asynchronous communication to update the directory infor- 
mation about where vertices are actually stored. The asynchronous communication overlaps well with the local computation of adding the vertices in the bContainer, thus providing good scalability up to a very large number of processors. There is only $36 \%$ increase in execution time for add_vertex in the dynamic pGraph as we scale from 4 to 16384 processors.

\section{Evaluation of Address Translation Mechanisms}

In this section we evaluate the performance of the three types of address translation mechanisms introduced in Section D: a static partition with a closed form solution mapping GIDs to bContainers, and distributed dynamic partitions with and without method forwarding. When method forwarding is not allowed, the partition fetches the GID mapping information using synchronous operations. When method forwarding is allowed, the method is asynchronously sent first to the location owning the directory which in turn forwards the method to the actual location where the element resides.

We evaluate the performance of the three partitions using a simple pGraph algorithm that finds source vertices (i.e., vertices with no incoming edges) in a directed graph. The algorithm traverses the adjacency list of each vertex and increments a counter on the target vertex of each edge. The communication incurred by this algorithm depends on the number of remote edges, i.e., edges connecting vertices in two different bContainers. We considered four graphs, all 2D tori, which vary according to the percentage of remote edges: $.03 \%, .33 \%, 3.4 \%$, and $50 \%$. This was achieved by having each processor hold a stencil of 1,500×1,500, $150 \times 15,000,15 \times 150,000$, $1 \times 2,250,000$, respectively.

Figure 51(a) provides a summary of the execution times for the different percentages of remote edges and different numbers of processors, where scalability can 


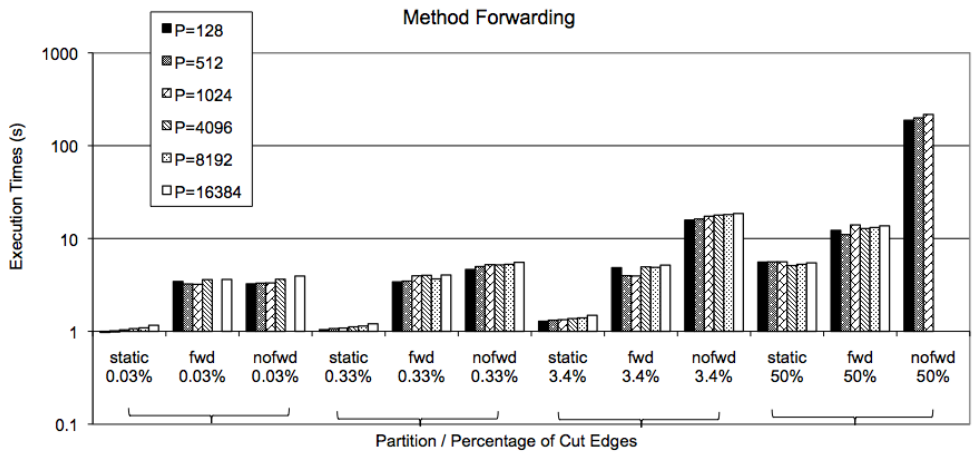

(a)

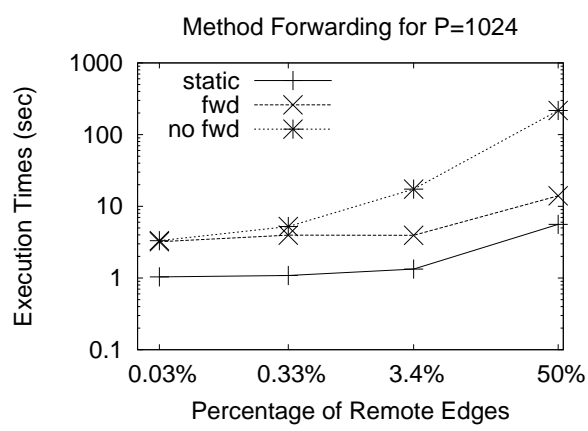

(b)

Fig. 51. Find sources in a directed pGraph using static, dynamic with forwarding and dynamic with no forwarding partitions. Execution times for graphs with various percentages of remote edges for (a) various processor counts and for (b) 1024 processors.

be appreciated together with the increasing benefit of forwarding as the percentage of remote edges increases. In Figure 51(b) we include results for the three approaches on all four types of graphs for 1024 processors. As can be seen, for the methods with no forwarding and synchronous communication, the execution time increases as the percentage of remote edges increases. The static method and the method with forwarding track one another and do not suffer as badly as the percentage of remote edges increases. This indicates that the forwarding approach can scale similarly to the optimized static partition.

In Figure 52 we show weak scaling experiments for all four graph types. While the scalability is good for all methods, the static partition is always superior and, again, it is seen that for the dynamic partitions, the benefit of method forwarding increases as the percentage of remote edges increases, though it is quite significant even if the percentage of remote edges is quite low (e.g., 3.4\%). When we use a 1x2250000 
stencil (50\% remote edges), partition_static is around 5 seconds, partition_fwd is 12 seconds, while partition nofwd is 190 seconds.

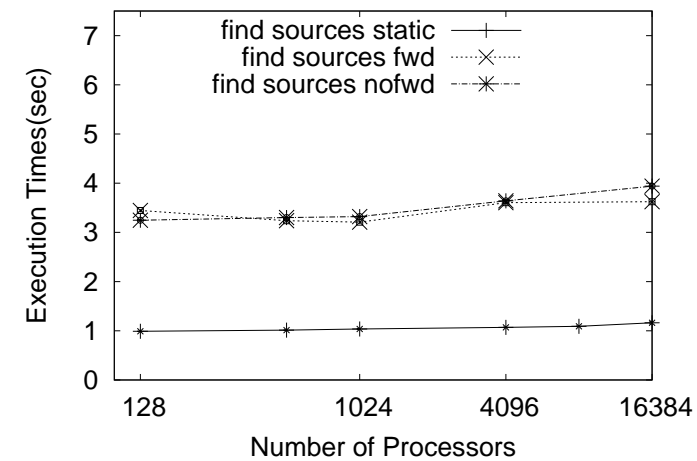

(a) $0.03 \%$ remote edges

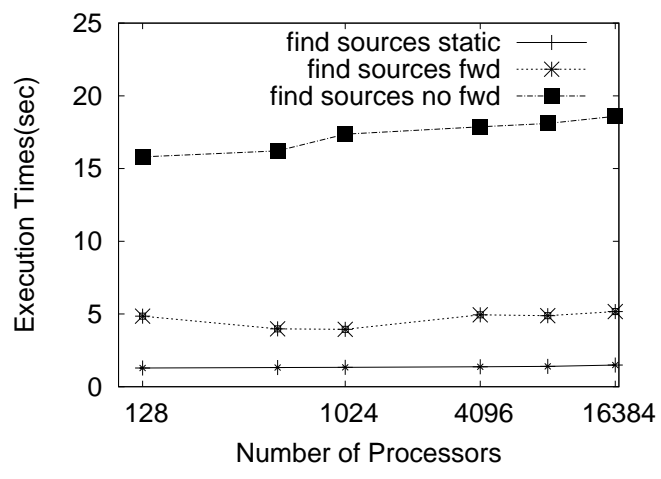

(c) $3.4 \%$ remote edges

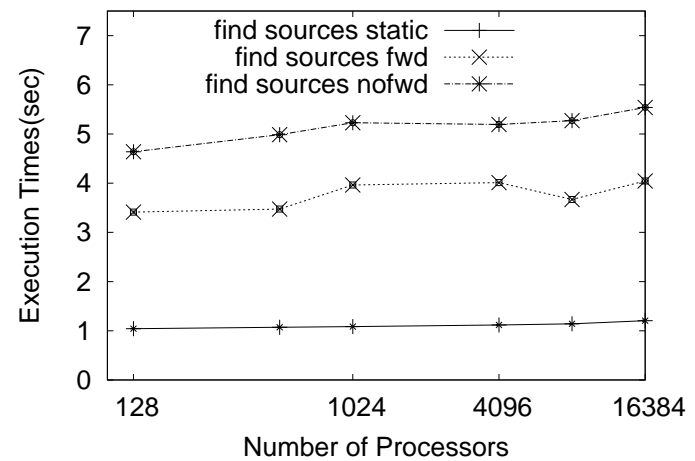

(a) $0.33 \%$ remote edges

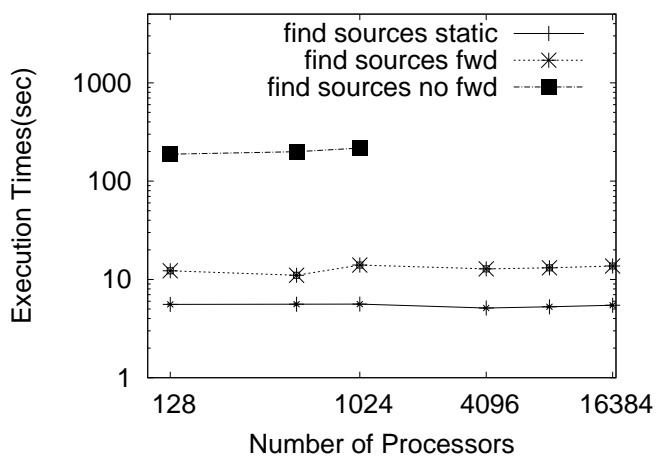

(c) $50 \%$ remote edges

Fig. 52. Comparison of various pGraph partitions. Execution times (weak scaling) for graphs with (a) $.03 \%$, (b) $.33 \%$, (c) $3.4 \%$, and (d) $50 \%$ remote edges. 


\section{3. pGraph Algorithms}

In this section we analyze the performance of several generic STL algorithms and pGraph specific algorithms for various input types and pGraph characteristics. In Figure 53 we show results for three generic STL algorithms. p_for_each applies to every vertex in the graph a functor that sets the vertex property which is a double to zero. The theoretical complexity for this operation is $O(|V| / P)$, where $|V|$ is the number of vertices in the graph and $P$ is the number of processors. p_accumulate accumulates all vertex properties. The theoretical complexity for this operation is $O(|V| / P+\log (P))$. The $\log (P)$ factor is due to the reduction performed. The p_max_weight find the edge with the maximum edge weight. Its complexity is $O(|E| / P)$, where $|E|$ is the total number of edges. We observe from the figure good scaling for all three algorithms for both static and dynamic pGraphs. The p_accumulate execution time slowly increases as we increase the number of processors and this is due to the reduction step. For all pGraphs considered the number of edges is bigger than the number of vertices and this is properly reflected in the graphs.

In Figures 54 and 55 we include results for graph specific algorithms on CRAY4 and P5-CLUSTER, respectively. The find_edges collects all edges with maximum edge weight into an output pList (SSCA2 benchmark); find_sources collects all vertices with no incoming edges into an output pList. find_sources takes as input a collection of vertices and performs graph traversals in parallel. The traversal proceeds in a DFS style. When a remote edge is encountered, a new task is spawned to continue the traversal on the location owning the target. The current traversal will continue in parallel with the spawned one. This is useful for example when we want to compute all vertices and edges accessible from a set of starting points. trim is another useful computation when computing cycles or strongly connected compo- 
CRAY: Static pGraph Algorithms Weak Scaling

SSCA2, 500K vertices, $11 \mathrm{M}$ edges/location

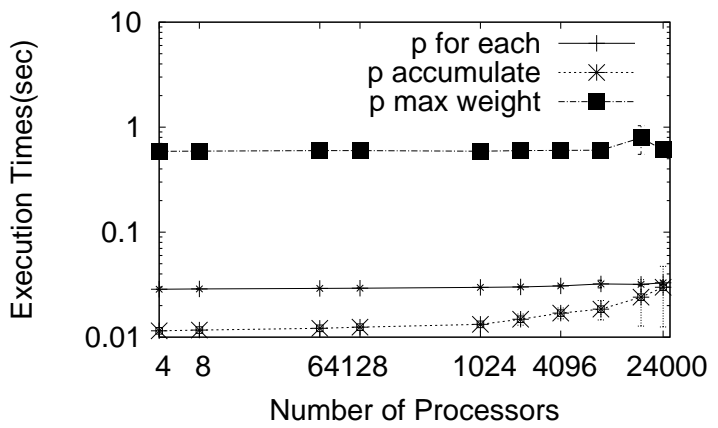

(a) SSCA Static

CRAY: Static pGraph Algorithms Weak Scaling Sparse Mesh,2.25M vertices,3M edges/location

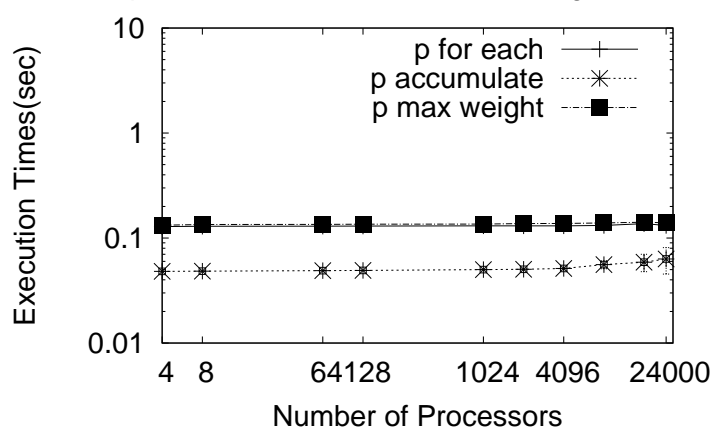

(c) $1500 \times 1500$ Mesh Static

CRAY: Static pGraph Algorithms Weak Scaling Sparse Mesh,2.25M vertices,3M edges/location

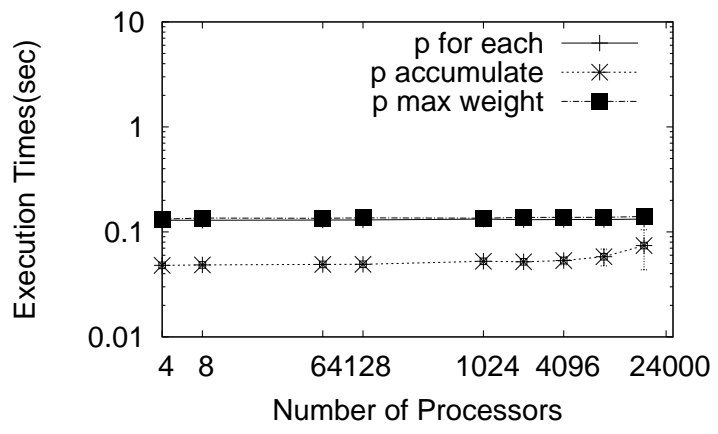

(e) $15 \times 150000$ Mesh Static
CRAY4: Dynamic pGraph Algorithms Weak Scaling SSCA\#2,500K vertices,23M edges/location

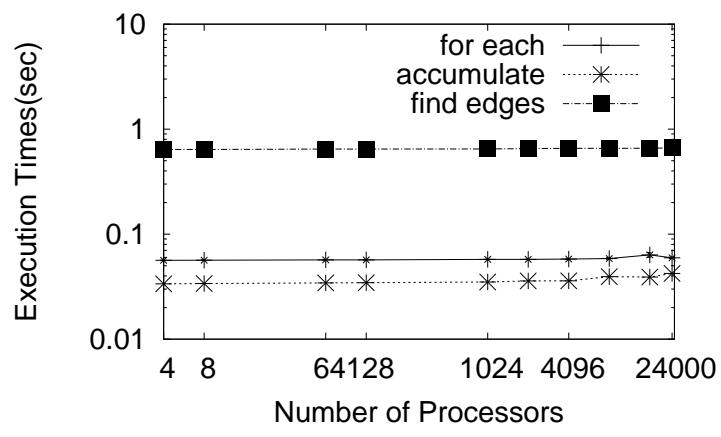

(b) SSCA Dynamic

CRAY: Dynamic pGraph Algorithms Weak Scaling Sparse Mesh,2.25M vertices,3M edges/location

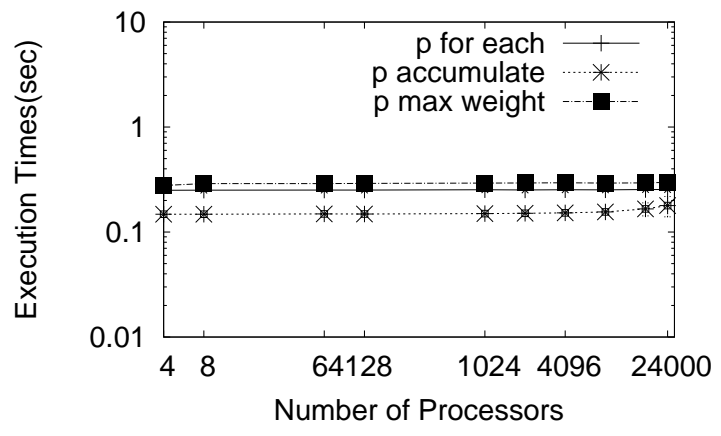

(d) $1500 \times 1500$ Mesh Dynamic

CRAY: Dynamic pGraph Algorithms Weak Scaling Sparse Mesh,2.25M vertices,3M edges/location

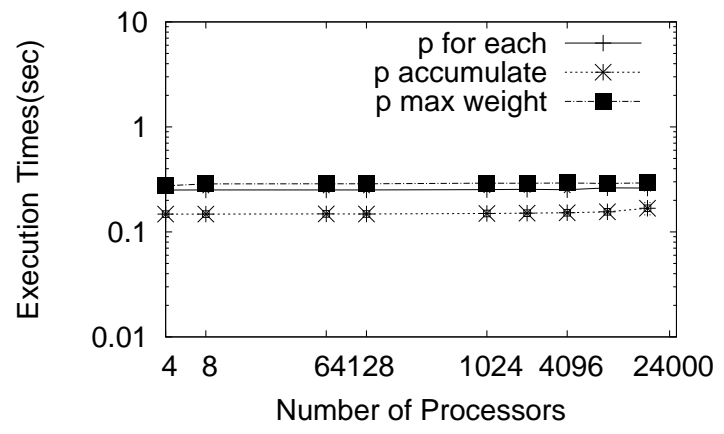

(f) $15 \times 150000$ Mesh Dynamic

Fig. 53. CRAY4: Execution times for different pGraph algorithms. Static versus dynamic pGraph comparison. The input is a sparse mesh or generated using the SSCA2 scalable generator with $500 \mathrm{~K}$ vertices per processor. 
CRAY4: Static pGraph Algorithms Weak Scaling SSCA2, 500K vertices, $11 \mathrm{M}$ edges/location

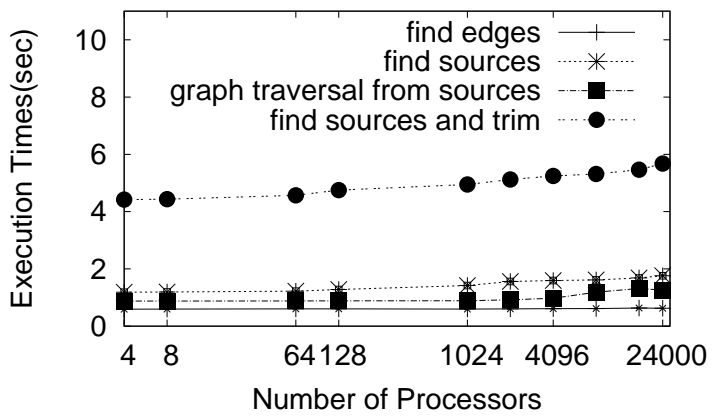

(a) SSCA Static

CRAY: Static pGraph Algorithms Weak Scaling Sparse Mesh,2.25M vertices,3M edges/location

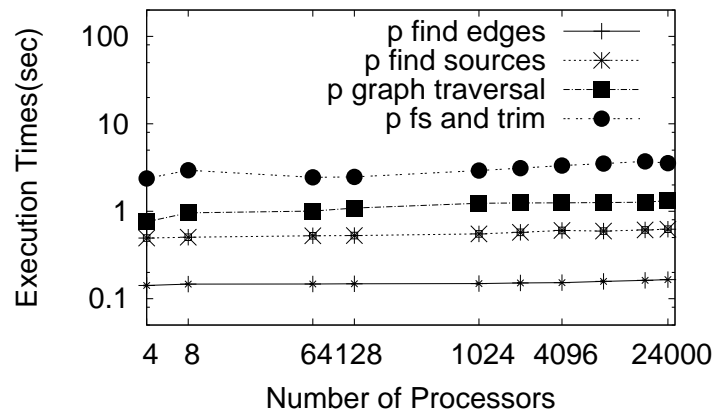

(c) $1500 \times 1500$ Mesh Static

CRAY: Static pGraph Algorithms Weak Scaling Sparse Mesh,2.25M vertices,3M edges/location

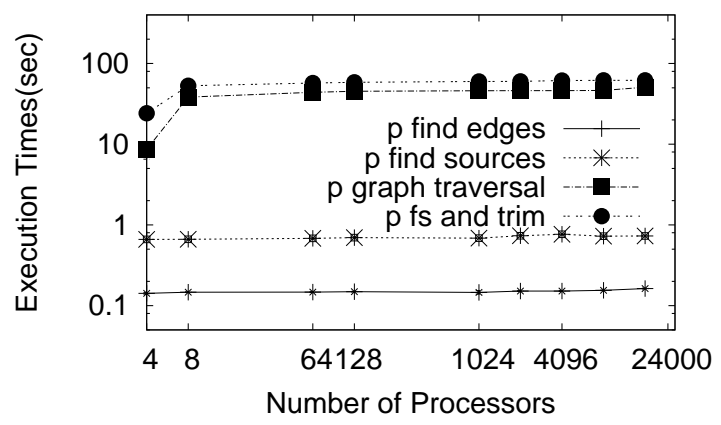

(e) $15 \times 150000$ Mesh Static
CRAY4: Dynamic pGraph Algorithms Weak Scaling SSCA2, 500K vertices, $11 \mathrm{M}$ edges/location

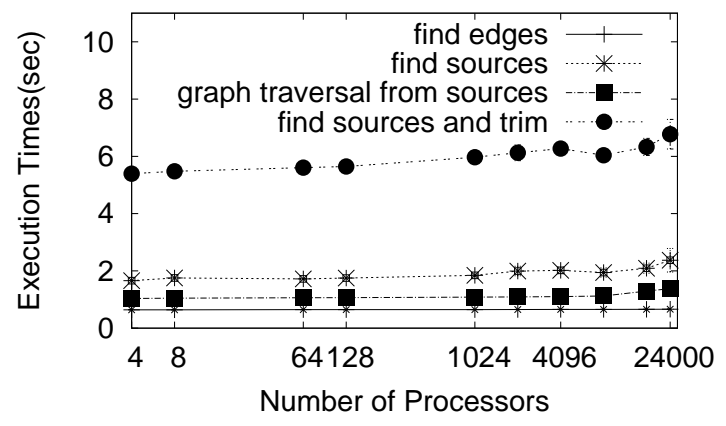

(b) SSCA Dynamic

CRAY: Dynamic pGraph Algorithms Weak Scaling Sparse Mesh,2.25M vertices,3M edges/location

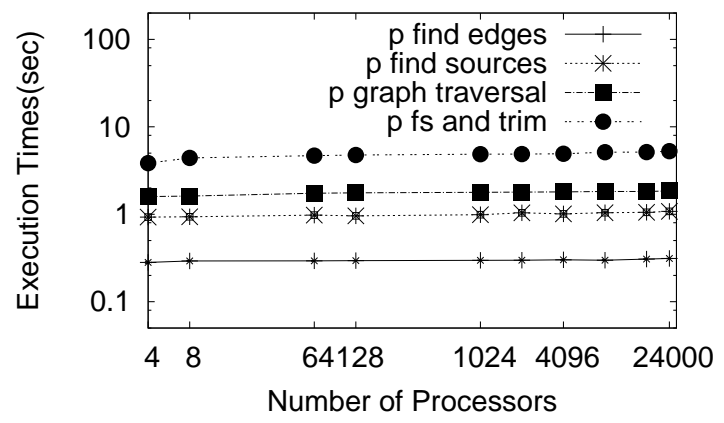

(d) $1500 \times 1500$ Mesh Dynamic

CRAY: Dynamic pGraph Algorithms Weak Scaling Sparse Mesh,2.25M vertices,3M edges/location

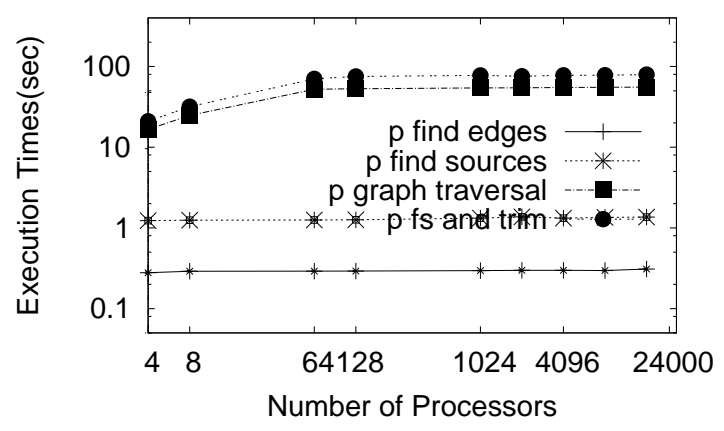

(f) $15 \times 150000$ Mesh Dynamic

Fig. 54. CRAY4: pGraph algorithms. Static versus dynamic pGraph comparison. The input is a sparse mesh or generated using the SSCA2 scalable generator with $500 \mathrm{~K}$ vertices per processor. 
P5: Static pGraph Algorithms Weak Scaling SSCA\#2, 500K vertices, $11 \mathrm{M}$ edges/location

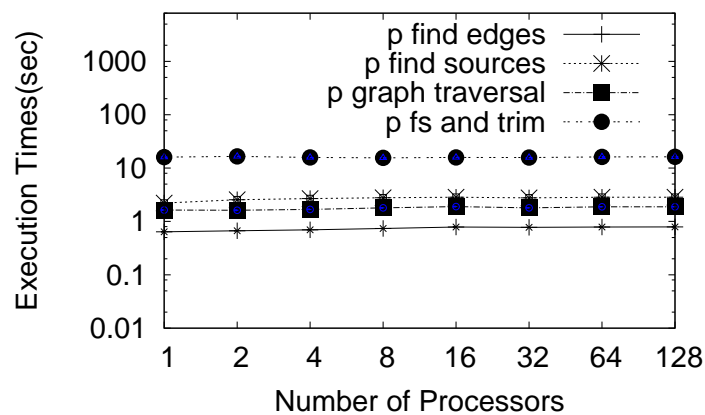

(a) SSCA Static

P5: Static pGraph Algorithms Weak Scaling Sparse Mesh,2.25M verts, $4.5 \mathrm{M}$ edges/location

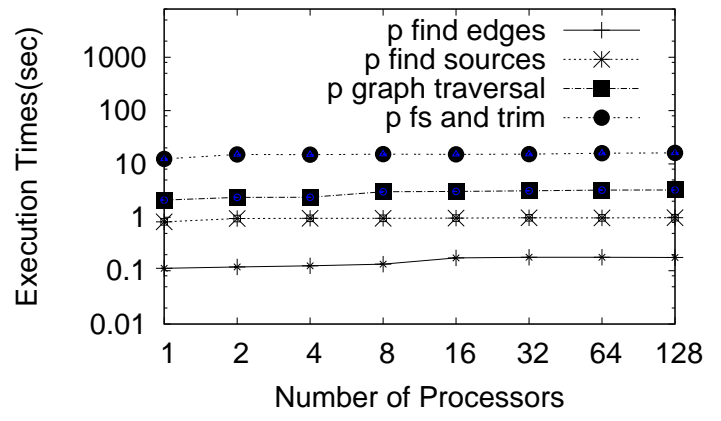

(c) $1500 \times 1500$ Mesh Static

P5: Static pGraph Algorithms Weak Scaling Sparse Mesh,2.25M verts,4.5M edges/location

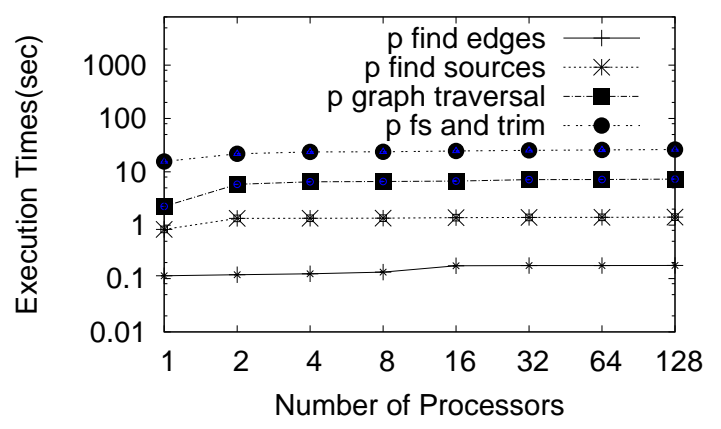

(e) $15 \times 150000$ Mesh Static
P5: Dynamic pGraph Algorithms Weak Scaling SSCA\#2, 500K vertices, $11 \mathrm{M}$ edges/location

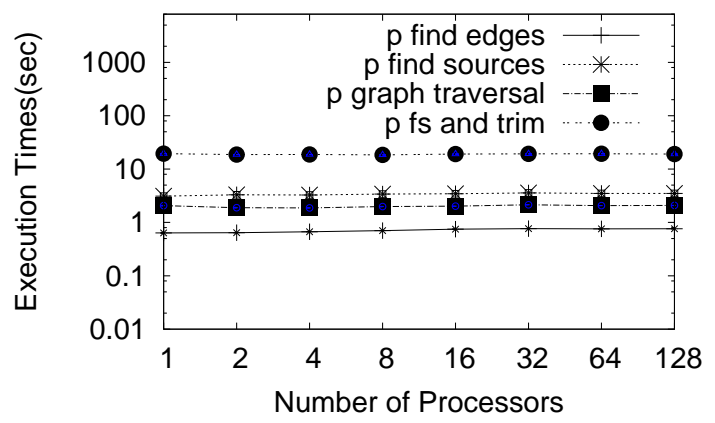

(b) SSCA Dynamic

P5: Dynamic pGraph Algorithms Weak Scaling Sparse Mesh,2.25M verts,4.5M edges/location

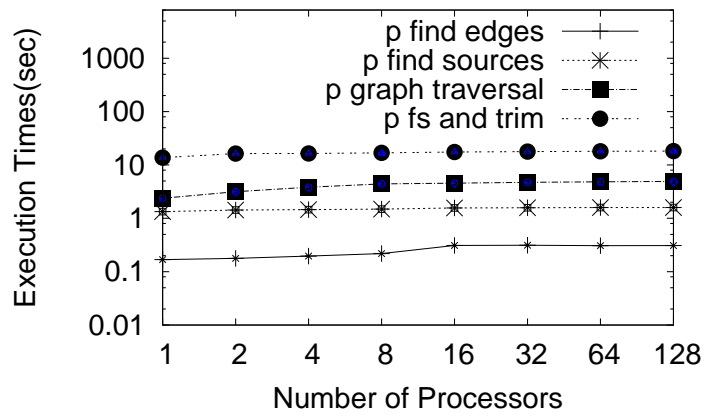

(d) $1500 \times 1500$ Mesh Dynamic

P5: Dynamic pGraph Algorithms Weak Scaling Sparse Mesh,2.25M verts,4.5M edges/location

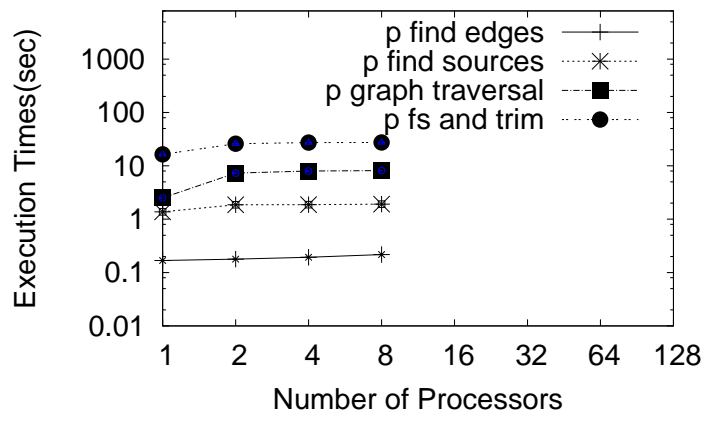

(f) $15 \times 150000$ Mesh Dynamic

Fig. 55. P5-CLUSTER: Execution times for different pGraph algorithms. Static versus dynamic pGraph comparison. The input is a sparse mesh or generated using the SSCA2 scalable generator with $500 \mathrm{~K}$ vertices per processor. 
nents. It computes the set of sources for a directed graph and removes all their edges, recursively continuing with the newly created sources. The process will stop when there are no more sources.

We run the algorithms on various input types including a sparse mesh and SSCA2 random graphs. In Figure 54(a), (b), and Figure 55(a), (b), weak scaling results are shown for SSCA2 for both static and dynamic pGraphs. The number of processors varied from 4 to 24000. For all algorithms considered, the static graph performed better due to the faster address resolution and std: : vector storage for vertices versus std: :hash_map. find_edges, a fully parallel algorithm, exhibits good scalability with less than $5 \%$ increase in execution time for both types of graphs on CRAY4. find_sources incurs communication proportional to the number of remote edges. The algorithms use two containers, traversing an input pGraph and generating an output pList. The traversal from sources and trim algorithm spawn new computation asynchronously as it reaches a remote edge. Additionally, the trim algorithm removes pGraph edges, which negatively impacts performance. The increase in execution time for the trim algorithm is $28 \%$ for static and $25 \%$ for dynamic pGraphs on CRAY4.

Figure 54(c), (d), (e) and (f) illustrate that the execution time of pGraph algorithms increases with the number of remote edges. When the $1500 \times 1500$ stencil is used the number of remote edges is small relative to the local ones and there is good communication computation overlap enabling the algorithms to scale up to a large number of processors. When the stencil used is $15 \times 150000$, the remote to local edges ratio is $3.4 \%$. The increased number of remote edges is reflected in the execution times of the algorithms because they incur communication proportional to the number of edges. Despite an increased execution time we observe that the algorithms scale well up to 24000 processors, proving that the STAPL pGraph data structure can be successfully used to solve very large graph problems. 


\section{Page Rank}

In this section we examine the performance of the page rank [12] algorithm. The algorithm performs a number of iterations and in each iteration, for all vertices, we update the ranks of all neighbor vertices based on the rank of the current vertex. The algorithm incurs communication proportional to the number of remote edges. In Figure 56 we show experimental results for two different meshes, one with $0.03 \%$ remote edges and one with $3.4 \%$ remote edges per location. The algorithm scales well as we scale the number of processors from 4 to 8192 , the communication cost being visible only on the larger number of processors.

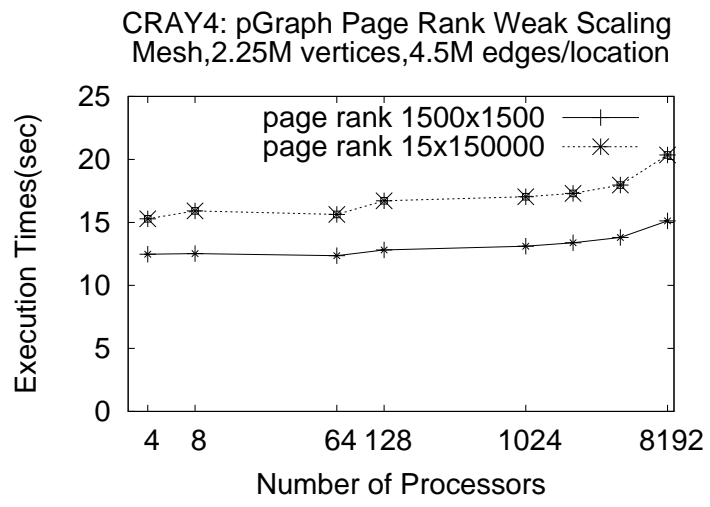

Fig. 56. Page rank for two different input meshes: 1500x1500 and 15x150000. 


\section{CHAPTER XII}

\section{ASSOCIATIVE PCONTAINERS*}

An associative container provides optimized methods for storing and retrieving data using keys. In STAPL, similar to STL [49], we consider the following six basic associative container concepts: simple, pair, sorted, hashed, unique and multiple. Simple specifies that the container will store only keys while pair means that the container will store pairs of keys and values. Sorted guarantees that the internal organization allows logarithmic time implementations for insert, delete and find operations, while hashed containers guarantee asymptotic constant time for these operations. In addition, traversing the data of a sorted associative container from begin to end guarantees that the elements are traversed in sorted order. Unique guarantees that all data elements have unique keys, while multi allows for duplicate keys. Each of these concepts specifies properties and interfaces, e.g., simple associative pContainer methods have keys in the interface (e.g., sets), while pair associative pContainers have methods with both keys and values (e.g., maps), hashed and sorted associative pContainers specify complexity requirements, and single or multi specify the semantics of the operations.

Based on this taxonomy, STAPL provides six associative pContainers that are compositions of the basic concepts (see Figure 57(b)): pSet (simple, sorted, unique), pMap (pair, sorted, unique), pMultiSet (simple, sorted, multiple), pMultiMap (pair, sorted, multiple), pHashMap (pair, hashed, unique), and pHashSet (simple, hashed,

*Part of the data reported in this chapter is reprinted with the kind permission of Springer Science+Business Media from "Associative parallel containers in STAPL," by G. Tanase, C. Raman, M. Bianco, N. M. Amato, and L. Rauchwerger, 2008. Lecture Notes in Computer Science, vol. 5234, pp. 156-171, Copyright 2008 by Springer. 


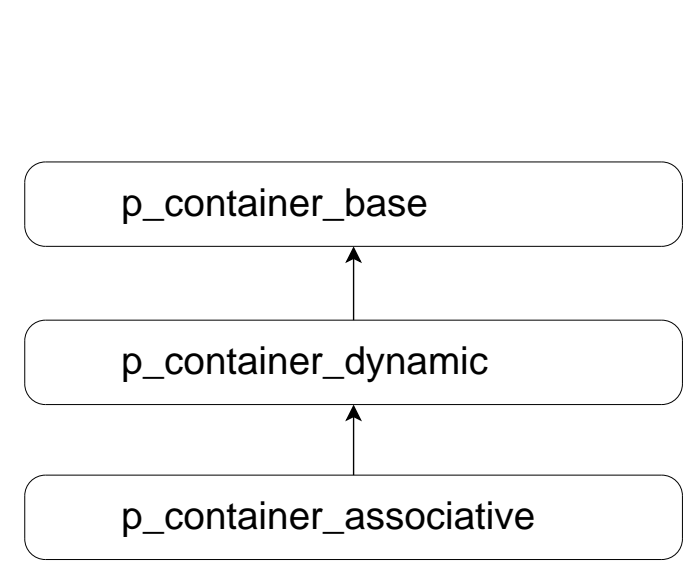

(a)

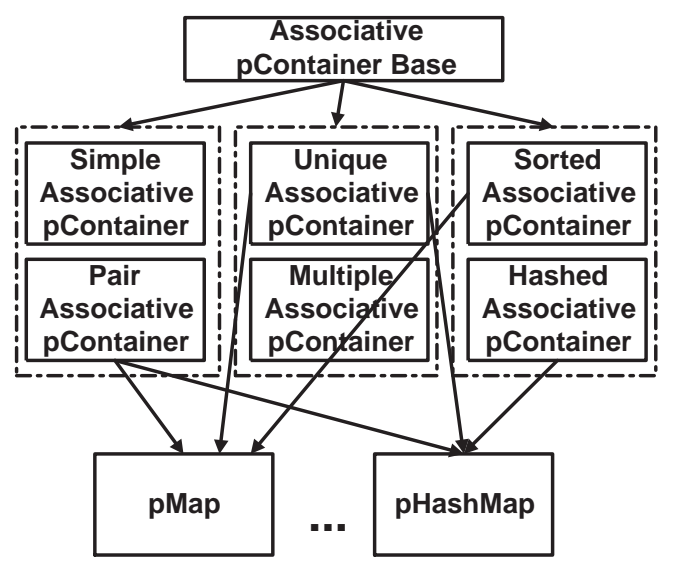

(b)

Fig. 57. Associative pContainer: (a) derivation from the framework base classes (b) associative pContainers internal hierarchy.

unique).

A. Associative pContainer Specification

The template declarations for the six STAPL associative pContainers are:

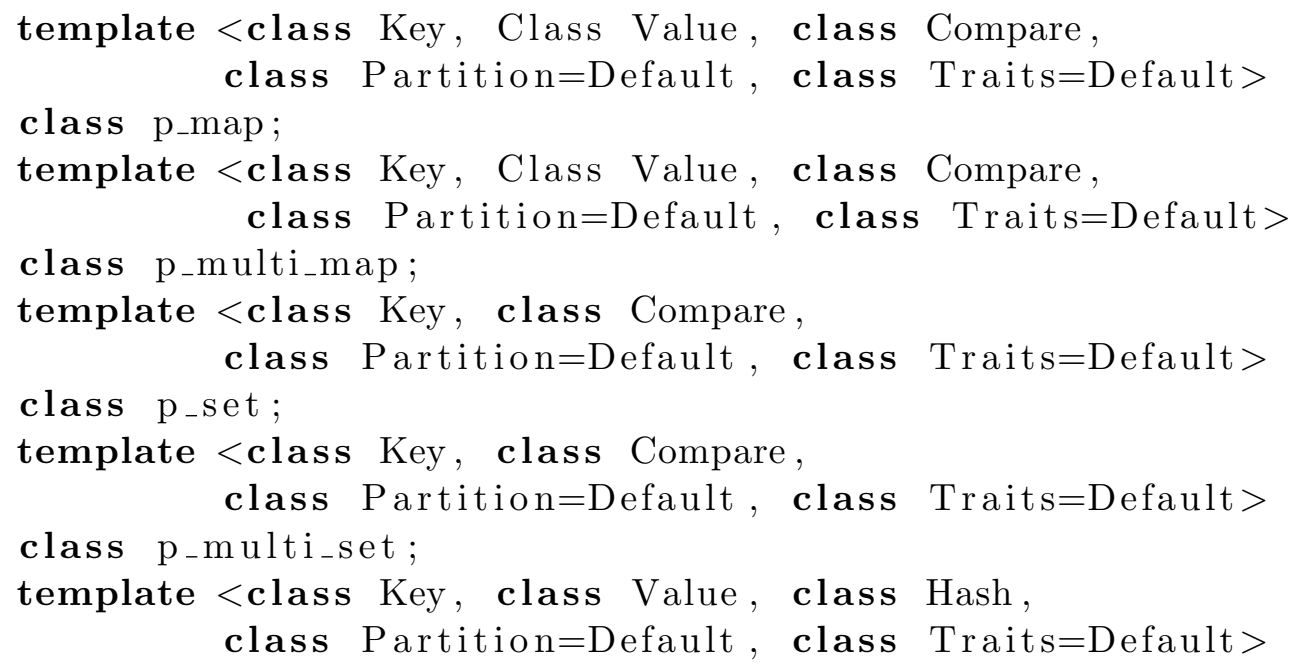


class p_hash_map;

template < class Key, class Hash

class Partition=Default, class Traits $=$ Default $>$

class p_hash_set;

The STAPL associative pContainers provide the generic specification (data types and methods) included in table XXVIII. The complexity of all element-wise methods is $O(\log (N))$ for sorted where $\mathrm{N}$ is the pContainer size, and amortized constant time for hashed.

Table XXVIII.: Associative pContainers interface.

\begin{tabular}{|l|l|}
\hline Template Arguments & Description \\
\hline Traits & $\begin{array}{l}\text { Traits to specify the low level base container } \\
\text { used and distribution features. }\end{array}$ \\
\hline Define Type & Description \\
\hline key_type & the type of the Key \\
\hline value_type & $\begin{array}{l}\text { the type of the Value (not available for sim- } \\
\text { ple associative) }\end{array}$ \\
\hline key_compare & $\begin{array}{l}\text { the type for key comparisons (not available } \\
\text { for hashed) }\end{array}$ \\
\hline Method & Description \\
\hline iterator insert(key[,value]) & $\begin{array}{l}\text { insert the (key, value) pair (no value for } \\
\text { simple associative). Return iterator point- } \\
\text { ing to inserted item. }\end{array}$ \\
\hline size_t erase(key) & $\begin{array}{l}\text { Erases all elements with key equal to } k . \text { Re- } \\
\text { turn number of erased elements. }\end{array}$ \\
\hline iterator find(key) & $\begin{array}{l}\text { Return an iterator pointing to an element } \\
\text { with key equal to } k \text { or end() if no such ele- } \\
\text { ment is found. }\end{array}$ \\
\hline $\begin{array}{l}\text { void insert_async(key[,value]), } \\
\text { erase_async(key) }\end{array}$ & $\begin{array}{l}\text { Non-blocking insert/erase (no value for } \\
\text { simple associative) }\end{array}$ \\
\hline key find_val(key) & $\begin{array}{l}\text { blocking operations returning values (in- } \\
\text { stead of iterators). }\end{array}$ \\
\hline
\end{tabular}


All STL equivalent methods require a return type, which in general translates into a synchronous (blocking) method. For this reason, we provide a set of asynchronous methods as part of the associative pContainer, e.g., insert_async and erase_async. These non-blocking methods allow for better communication/computation overlap and enable the STAPL RTS to aggregate messages to reduce the communication overhead.

We also introduce new associative pContainer methods that return values instead of iterators. These methods are provided because in STAPL a remote call will be issued when an iterator to a remote element is dereferenced. Hence, if a programmer knows the value will be needed, they should use the method that returns a value rather than the method that returns an iterator.

\section{B. Associative pContainer Design and Implementation}

In this section, we describe the pList modules used for storage and data distribution information.

bContainer: We have implemented the associative pContainer bContainers by extending the corresponding sequential container (typically STL containers) with functionality needed to implement domain instances.

Global Identifier (GID): For a simple associative pContainer the GID associated with each element is a key, whereas it is a (key, $m$ ) pair for a multi associative pContainer, where $m$ is an integer used to manage multiplicity

Domain and Domain Instance: The domain of the associative pContainer is given by the range of possible keys the pContainer can hold. For example, for a pMap over strings the domain can be the set of all possible strings or the set of all possible strings between two boundaries according to some order relation (e.g., lexicographical 


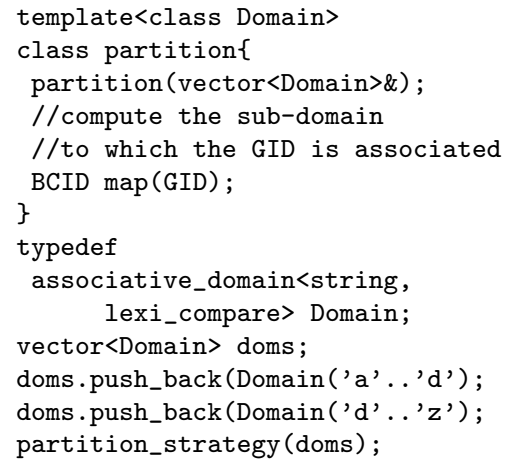

Fig. 58. Value based partition for sorted associative pContainers.

order). At any instant, there is only a finite set of elements in the container. The GIDs associated with these elements is the domain instance of the pContainer. For example AssociativeDomain<string>(' $a$ ' , ' $\mathrm{k}$ ') is a domain comprising all strings that are greater than ' $a$ ' and strictly smaller than ' $\mathrm{k}$ ' according to the lexicographical order. A domain instance corresponding to the previously defined associative domain might be $\{$ 'a', 'aa', 'abc', 'joe' $\}$.

Data Distribution: The data distribution manager uses (i) a partition to decide for every key in the domain to which sub-domain it has been allocated, and (ii) a partition-mapper to decide to which location each sub-domain has been allocated.

Partition: Associative pContainers are dynamic containers supporting concurrent additions and deletions of elements, thus the corresponding partitions have to provide functionality to add or delete GIDs to/from the corresponding domain instance or, e.g., to perform repartitions to ensure load balance. The default partition implemented by STAPL sorted associative pContainers is a static blocked partition over the key space. Users can provide additional partitions for associative 
pContainers by explicitly enumerating the corresponding sub-domains as illustrated in Figure 58. For a hashed associative pContainer, the partition can be specified by providing a hash function that will map a key to a sub-domain ID (e.g., hash(key) mod num_subdomains).

Partition Mapper: Similar to other STAPL pContainers associative containers can use any of the following partition mappers: partition_mapper_generic, partition

mapper_blocked, partition_mapper_cyclic or partition_mapper_identity.

Associative pContainer pViews: pViews are defined as the accessors for the data elements stored in the pContainer. The pViews over pMap, pMultiMap, and pHashMap support mutable iterators over data. This allows the value field to be modified. The others (pSet, pMultiSet, and pHashSet) provide read only pViews with const iterators.

Associative pContainer Base Class: To automate and standardize the process of developing associative pContainers, we designed a common base that is responsible for maintaining the data and the distribution manager. The associative pContainer base is generic and uses template parameters and traits classes to tailor the data structure to the user's needs. Each basic associative concept (simple, pair, unique, multi, sorted, hashed) is implemented as a class derived from the associative pContainer base to provide the specified functionality and enforce the required properties. Each associative pContainer (e.g., pMap), inherits from three corresponding classes as depicted in Figure 57(b).

The time for performing the operation on the bContainers is logarithmic or amortized constant time for sorted and hashed pContainers, respectively. The memory overhead depends on the partition used. A blocked partition for a sorted pContainer requires space proportional to the number of sub-domains, while for a 


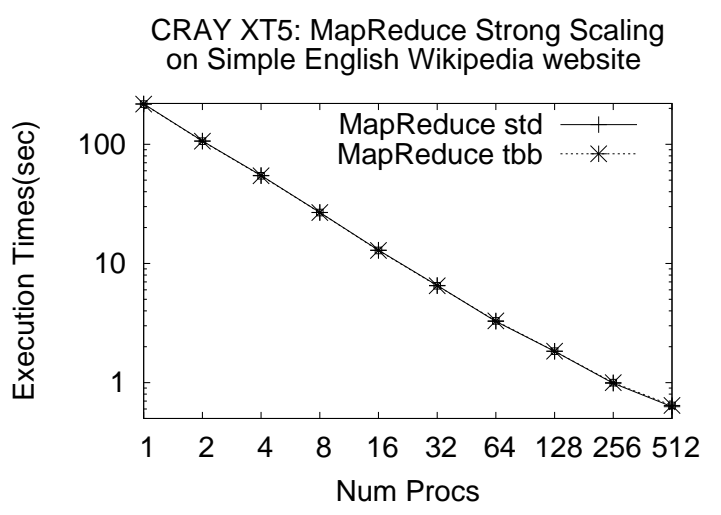

(a) MapReduce Execution Times

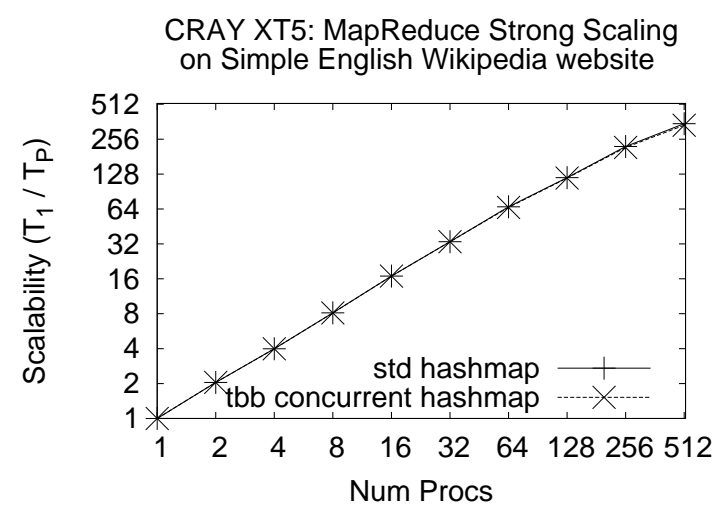

(b) MapReduce Scalability

Fig. 59. MapReduce used to count the number of occurrences of every word in Simple English Wikipedia website (1.5GB).

hashed partition the overhead is constant in each location. Different partitions, with more complex invariants, may incur different computational and memory overheads. C. Performance Evaluation

In this section, we evaluate the scalability of the parallel methods using a map reduce application and we evaluate two generic pAlgorithms, p_for_each and p_accumulate.

\section{MapReduce}

Here we examine the performance of a simple application implemented on top of a MapReduce framework developed in STAPL. The MapReduce uses the pHashMap[64], a dynamic associative pContainer. The application splits the input data across the available processors and first applies the map and reduce functions locally. After the local MapReduce phase is finished, the processor asynchronously inserts its locally reduced data into a pHashMap. The asynchronous insert calls the user's reduce func- 
tion if the key being inserted already exists in the pHashMap. The communication and data distribution is taken care of entirely by the pContainer. We ran a computation that computes the mutliplicity of each word in a $1.5 \mathrm{~GB}$ text input of the Simple-English Wikipedia website (simple.wikipedia.org). Because the input size was fixed and given, we include a strong scaling study where we measure the time taken to compute the multiplicity for all input words on CRAY5. In Figure 59 we show experiments corresponding to two different pHashMap storages, one using the STL std: : hash_map and another using the TBB concurrent hash map. We observe that the application scales well up to 512 processors and there is no noticeable difference when using different storages. The slowdown on 256 and 512 processors is due to the small computation performed per processor relative to the communication required to insert the data into the pHashMap.

\section{Generic Algorithms}

In this section, we examine the performance of various generic parallel algorithms operating on a linearization of the associative pContainer's data. Figure 60 shows the performance for p_for_each when operating on a single element pView defined over the pMap. As we scale the number of processors from 4 to 16384 we observe good scalability for all three algorithms considered: p_for_each, p_accumulate, and finding the maximum value in the container. 


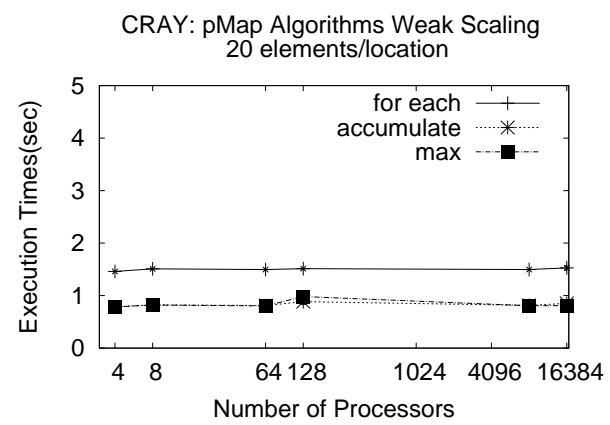

Fig. 60. CRAY4: Scalability for generic algorithms when using associative pContainers. 


\section{CHAPTER XIII}

\section{PCONTAINER COMPOSITION EVALUATION}

In Chapter V, Section $\mathrm{C}$ we introduced the pContainer composition as one of the main mechanisms for extending our library with custom data structures. We argue that composition helps increase the programmer productivity. Instead of directly building a complex pContainer, the programmer can compose one from the basic pContainers available in the PCF and shorten the development and debug time. For example, multi-dimensional arrays can be expressed as the composition of pArrays or pMatrices or a large scale distributed pGraph can be expressed as a pList of vertices where each vertex stores another pList of edges. In this section, we include experimental results to study the performance overhead of composed data structures relative to custom made ones.

For this comparison, we use a simple application that computes the minimum element in each row of a matrix using a pMatrix pContainer (which is available in the PCF library), a composed pArray of pArrays, and a composed pList of pArrays. The algorithm code is the same for all pContainers used, due to the access abstraction mechanism provided by STAPL pViews. It calls a parallel for_each on each row, and within each row, a map-reduce to compute the minimum value. The code for the algorithm is shown in Figure 61. We measure also the time to create and initialize the storage. The pMatrix allocates the entire structure in a single step (Figure 61, line 16), while the pArray of pArrays allocates the outer structure first (Figure 61, line 14) and subsequently allocates the nested pArrays in parallel using a parallel for_each (Figure 61, line 20). In Figure 61, line 23 a parallel for_each is invoked on a pView defined over the elements of the outer pArray. The functor of the parallel for each contains a nested pAlgorithm invocation (find minimum) that 


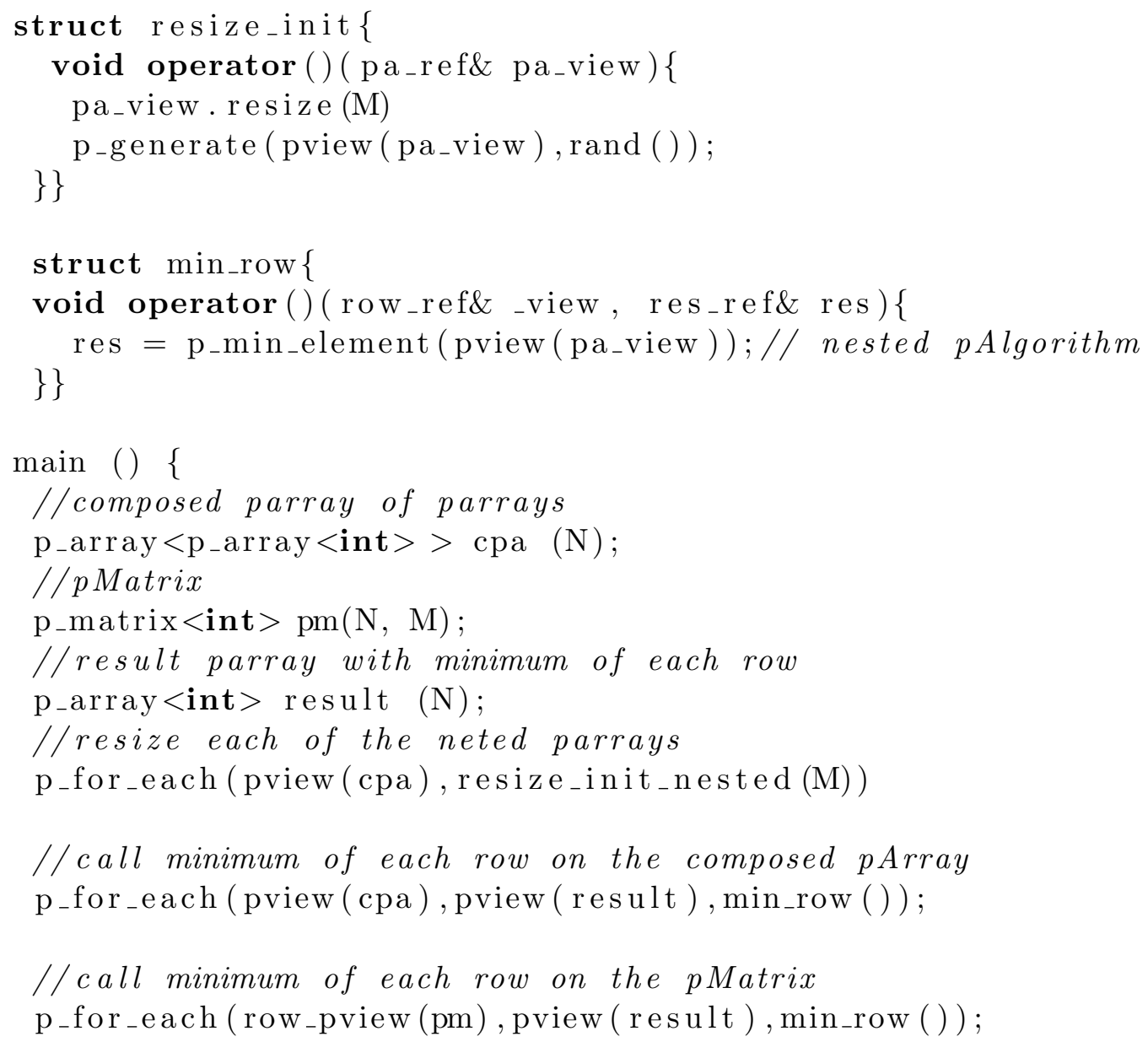

Fig. 61. Example of pContainer composition and nested pAlgorithm invocation.

is applied to each of the nested pArrays. In Figure 61, Line 26 the exact code used for computing the minimum of each nested pArray is used to compute the minimum of each row of the pMatrix by using appropriate pViews. This time the parallel for_each is invoked over a rows pView defined over pMatrix data and the functor to be applied to each row contains a nested parallel minimum algorithm. This simple 


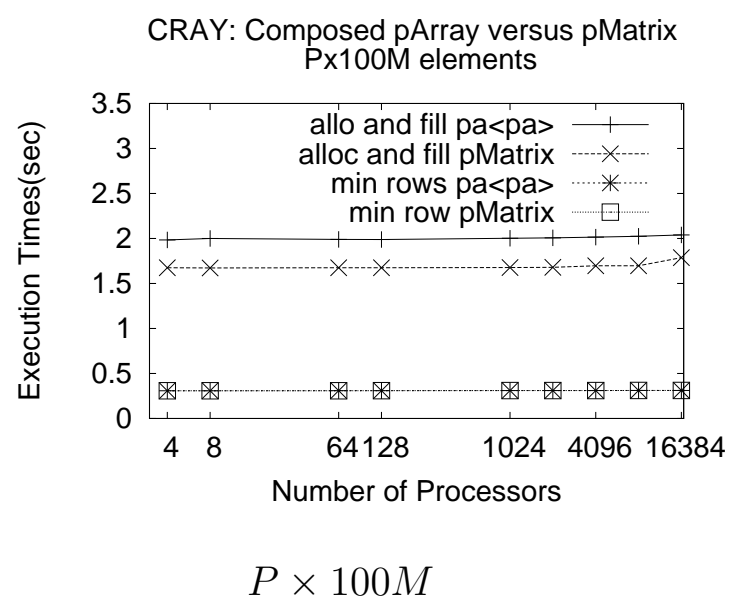

(a)

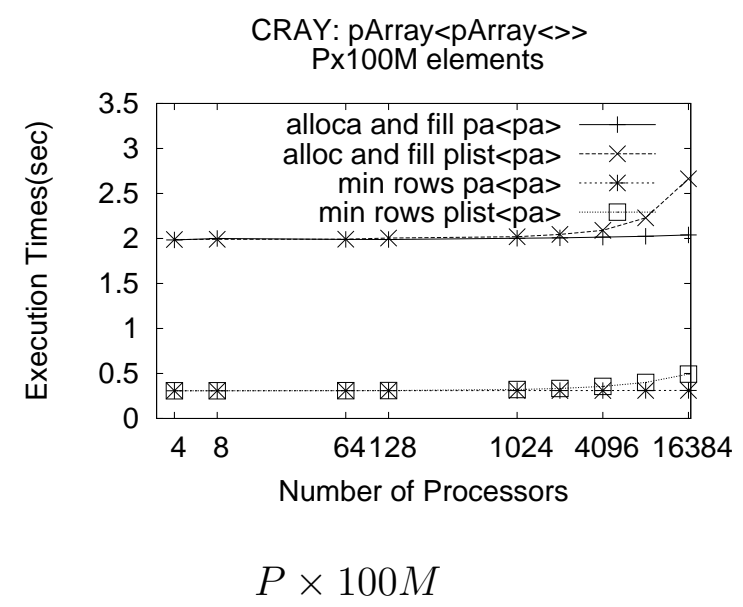

(b)

Fig. 62. Comparison of pArray<pArray<>> $(p a<p a>)$, pList<pArray<>> $($ plist $<p a>$ ) and pMatrix on computing the minimum value for each row of a matrix. Weak scaling experiment with $P \times 100 M$ elements. For the composed pContainer the outer pContainer is of size $P$ and the inner pContainer is of size 100M (a) pArray<pArray<>> versus pMatrix: pArray<pArray<>> takes longer to initialize while the algorithm executions are very similar (b) pArray<pArray<>> versus plist<pArray<>>: The plist<pArray<>> has additional overhead when creating the pViews over its data

example shows both the benefits of composition and the abstraction power provided by the pViews that allows users to easily assemble a parallel application.

In Figure 62(a) we include, for CRAY4, the execution times for allocating and initializing the composed pArray and the pMatrix and the times to run the min-ofeach-row algorithm, in a weak scaling experiment. The input used is a $P \times 100 M$ matrix where $P$ is the number of processors. In all experimens considered in this section the nested pArrays store their data on a single location and the nested pAlgorithms are executed by a single processor. These are preliminary results of the prototype 
of this mechanism. As expected, the pArray of pArrays allocation and initialization time is higher than that for a pMatrix. The time for the composed pArray includes the time of executing a parallel algorithm. The time for min-of-each-row algorithm, is very similar for the two data structures and scales well up to 16384 processors.

In Figure 62(b) we compare two composed pContainers. The composed pArray described in the previous paragraph and a composed pList of pArrays. In this case we observe similar times for the initialization of the data and computing the minimum for the low processor counts. While increasing the number of processors both the time for initialization and the time to run the algorithm increase for the composed pList much faster than the composed pArray. This is mainly due to the overhead of creating the pView abstractions on top of the pList container. Some of the overhead in the pView creation is subject to further research.

While we cannot state with certainty that our PCF allows for efficient composition (no additional overhead) for any combination of pContainers, the presented experiments indicate it is possible. 


\section{CHAPTER XIV}

\section{CONCLUSION AND FUTURE WORK}

In this dissertation, we presented the STAPL Parallel Container Framework (PCF), an infrastructure to facilitate the development of parallel and concurrent data structures. The salient features of this framework are: (a) a set of classes and rules to build new pContainers and customize existing ones, (b) mechanisms to generate wrappers around any sequential or parallel data structure, enabling its use in a distributed, concurrent environment and their use in cooperation with other libraries, (c) support for the (recursive) composition of pContainers into nested, hierarchical pContainers which can support arbitrary degrees of nested parallelism and (d) a library of basic pContainers constructed using the PCF as initial building blocks. We have shown how we have implemented a shared object view of the pContainers on distributed systems in order to relieve the programmer from managing and dealing with the distribution explicitly, unless so desired. The PCF allows users to customize its pContainers and adapt to dynamic and irregular environments, e.g., a pContainer can dynamically change its data distribution or adjust its thread safety policy to optimize the access pattern of the algorithms accessing the elements. Alternatively, the user can request certain policies and implementations which can override the provided defaults or adaptive selections. The PCF is an open ended project where users can add features as well as to the library and thus continuously improve the PCF's performance and utility.

Our experimental results on a very large parallel machine available at NERSC and a Power 5 cluster at Texas A\&M University supercomputing center show that pContainers provide good scalability for both static and dynamic pContainers.

The pContainer framework we developed enables a large number of new research 
directions that can be further pursued. First, our research will help users be more productive while developing new pContainers tuned for specific applications. For example motion planning [68] applications use a roadmap as their main data structure which is a natural extension of the graph. Using the PCF researchers in this area are provided with automatic support for parallelism by deriving the roadmap from the pGraph pContainer. Other applications such as particle transport use regular or arbitrary discretizations of the space called grids which can be naturally expressed as extensions of the pGraph data structure. We envision that for applications like this and numerous others, deriving data structures from base classes already provided by a library will be an important boost for user productivity.

Implementing various thread safety policies is another dimension that can be further exploited in our framework. The framework provides the proper interfaces for the thread safety manager as described in Chapter VI. Additionally, a set of predefined implementations are available but they have been minimally evaluated due to current limitations of the STAPL runtime system. With multithreading support available where multiple threads can be active within one locations all this functionality can be exercised and novel solutions proposed.

We described in Chapter VII the default relaxed memory consistency model provided by the pContainers developed in our framework. We have chosen the current model as it provides a good trade off between programmability and performance. However as mentioned in Chapter VII, Section E, other memory models more restrictive or more relaxed are possible. An interesting research direction is to study alternative models and evaluate their impact on productivity and performance.

pContainer composition as described in Chapter IV, Section C and Chapter XIII is a novel feature proposed in our framework as a modality to express new data structures. The composition opens a large number of research directions that require 
further exploration. These include deciding what is the optimal data distribution at different levels of the hierarchy, how data can be accessed by nested pAlgorithms and how composed pViews can be defined on data of a composed pContainer.

Another research dimension that our framework enables is the possibility to adapt a data structure to specific applications and architectures. We mentioned throughout this thesis that one of the major design goals of the library is to allow users to specialize functional modules of a pContainer by implementing well defined interfaces. Adaptivity will allow a pContainer to select among various modules with similar functionality. For example, different partitions and distributions may be available for a particular pContainer and currently the user decides which one to use. We envision that a pContainer can be augmented with the necessary support to perform the selection automatically.

In addition to providing users with a very large number of data structures, thus improving productivity, we believe that STAPL and the PCF are flexible infrastructures allowing researchers to experiments with data structures as a whole or with the individual functional modules that make up a parallel data structure. 


\section{REFERENCES}

[1] S. V. Adve and K. Gharachorloo, "Shared memory consistency models: A tutorial," IEEE Computer, vol. 29, no. 12, pp. 66-76, 1996.

[2] S. V. Adve and H. J. Boehm, "Memory models: A case for rethinking parallel languages and hardware," Commun. ACM, vol. 53, no. 8, pp. 90-101, 2010.

[3] S. V. Adve and M. D. Hill, "Weak ordering - a new definition," In Proc. of the 17th annual Int. Symposium on Computer Architecture, New York, NY, USA, 1990, pp. 2-14.

[4] P. An, A. Jula, S. Rus, S. Saunders, T. Smith, G. Tanase, N. Thomas, N. Amato, and L. Rauchwerger, "STAPL: A standard template adaptive parallel C++ library," In Proc. of the Int. Workshop on Advanced Compiler Technology for High Performance and Embedded Processors (IWACT), Bucharest, Romania, Jul 2001, pp. 37-46.

[5] P. An, A. Jula, S. Rus, S. Saunders, T. Smith, G. Tanase, N. Thomas, N. Amato, and L. Rauchwerger, "STAPL: An adaptive, generic parallel $\mathrm{C}++$ library," In Int. Workshop on Languages and Compilers for Parallel Computing, in Lecture Notes in Computer Science, vol. 2624, pp. 195-210, 2003.

[6] H. Attiya and J. Welch, Distributed Computing: Fundamentals, Simulations and Advanced Topics, London: McGraw-Hill, 1998.

[7] D. A. Bader and K. Madduri, "Design and implementation of the hpcs graph analysis benchmark on symmetric multiprocessors," In The 12th Int. Conf. on High Performance Computing (HiPC 2005), pp. 465-476. Springer, 2005. 
[8] G. Bikshandi, J. Guo, C. Praun, G. Tanase, B. B. Fraguela, M. J. Garzaran, D. Padua, and L. Rauchwerger, "Design and use of htalib: A library for hierarchically tiled arrays," In Int. Workshop on Languages and Compilers for Parallel Computing, in Lecture Notes in Computer Science, vol. 4382, pp. 17-32, 2007.

[9] G. Bikshandi, J. Guo, D. Hoeflinger, G. Almasi, B.B. Fraguela, M.J. Garzaran, D. Padua, C. Praun, "Programming for parallelism and locality with hierarchically tiled arrays," In Proc. ACM SIGPLAN Symp. Prin. Prac. Par. Prog., New York, NY, 2006, pp. 48-57.

[10] G. Blelloch, "NESL: A nested data-parallel language," Dept. Comp. Sci., Carnegie Mellon Univ., Pittsburgh, PA, Tech. Rep., CMU-CS-95-170, 1993.

[11] G. E. Blelloch, S. Chatterjee, J. C. Hardwick, J. Sipelstein, and M. Zagha, "Implementation of a portable nested data-parallel language," In Proc. ACM SIGPLAN Symp. Prin. Prac. Par. Prog., 1993, pp. 102-111.

[12] S. Brin and L. Page, "The anatomy of a large-scale hypertextual web search engine," Comput. Netw. ISDN Syst., vol. 30, no. 1-7, pp. 107-117, 1998.

[13] A. Buss, A. Fidel, Harshvardhan, T. Smith, G. Tanase, N. Thomas, X. Xu, M. Bianco, N. M. Amato, and L. Rauchwerger, "The STAPL pView," In Int. Workshop on Languages and Compilers for Parallel Computing, Houston, TX, 2010.

[14] A. Buss, A. Fidel, Harshvardhan, T. Smith, G. Tanase, N. Thomas, X. Xu, M. Bianco, N. M. Amato, and L. Rauchwerger, "The STAPL pView," Dept. Comp. Sci., Texas A\&M Univ., College Station, TX, Tech. Rep., TR10-001, July 2010. 
[15] A. Buss, T. Smith, G. Tanase, N. Thomas, M. Bianco, N. M. Amato, and L. Rauchwerger, "Design for interoperability in STAPL: pMatrices and linear algebra algorithms," In Int. Workshop on Languages and Compilers for Parallel Computing, in Lecture Notes in Computer Science, vol. 5335, pp. 304-315, July 2008.

[16] A. Buss, Harshvardhan, I. Papadopoulos, O. Pearce, T. Smith, G. Tanase, N. Thomas, X. Xu, M. Bianco, N. M. Amato and L. Rauchwerger "STAPL: Standard template adaptive parallel library," In Proc. of the 3rd Annual Haifa Experimental Systems Conf., pp. 1-10, 2010.

[17] D. Callahan, B. L. Chamberlain, and H. P. Zima, "The cascade high productivity language," In The Ninth Int. Workshop on High-Level Parallel Programming Models and Supportive Environments, vol. 26, pp. 52-60, 2004.

[18] P. Charles, C. Grothoff, V. Saraswat, C. Donawa, A. Kielstra, K. Ebcioglu, C. Praun, and V. Sarkar, "X10: An object-oriented approach to non-uniform cluster computing," In Proc. of the 20th annual ACM SIGPLAN Conf. on Object-Oriented Programming, Systems, Languages, and Applications, New York, NY, 2005, pp. 519-538.

[19] D. Culler, A. Dusseau, S. C. Goldstein, A. Krishnamurthy, S. Lumetta, T. Eicken, and K. Yelick, "Parallel programming in Split-C," In Int. Conf. on Supercomputing, November 1993.

[20] D. Dechev, P. Pirkelbauer, and B. Stroustrup. Lock-free dynamically resizable arrays. In Proc. of OPODIS, Bordeaux, France, 2006, pp. 142-156.

[21] J. Desouza and L. V. Kale, "MSA: Multiphase specifically shared arrays," In 
Int. Workshop on Languages and Compilers for Parallel Computing, in Lecture Notes in Computer Science, vol. 3602, pp. 268-282, 2005.

[22] R. E. Diaconescu and H. P. Zima, "An approach to data distributions in chapel," Int. J. of High Performance Computing Applications, vol. 21, no. 3, pp. 313-335, 2007.

[23] M. Dubois, C. Scheurich, and F. Briggs, "Memory access buffering in multiprocessors," In ISCA '98: 25 years of the Int. Symposium on Computer Architecture, New York, NY, 1998, pp. 320-328.

[24] H. Gao, J. F. Groote, and W. H. Hesselink. Almost wait-free resizable hashtables. 18th International Parallel and Distributed Processing Symposium, Santa Fe, New Mexico, 2004.

[25] T. El-Ghazawi, W. Carlson, T. Sterling, and K. Yelick, UPC: Distributed SharedMemory Programming. Hoboken, NJ: Wiley-Interscience, 2003.

[26] M. Greenwald. Two-handed emulation: how to build non-blocking implementations of complex data-structures using DCAS. In Proc. of the Twenty-first Annual Symposium on Principles of Distributed Computing (PODC), Monterey, Ca, 2002, pp. 260-269.

[27] M. Fomitchev and E. Ruppert, "Lock-free linked lists and skip lists," In Proc. Symp. on Princ. of Distributed Programming, New York, NY, 2004, pp. 50-59.

[28] P. Gottschling, D. S. Wise, and M. D. Adams, "Representation-transparent matrix algorithms with scalable performance," In Proc. Int. Conf. on Supercomputing, Seattle, Washington, 2007, pp. 116-125. 
[29] D. Gregor and A. Lumsdaine, "Lifting sequential graph algorithms for distributed-memory parallel computation," In Proc. of the 20th annual ACM SIGPLAN Conf. on Object-Oriented Programming, Systems, Languages, and Applications, New York, NY, 2005, pp. 423-437.

[30] D. Gregor and A. Lumsdaine, "The parallel BGL: A generic library for distributed graph computations," In Proc. of Workshop on Parallel Object-Oriented Scientific Computing, July 2005.

[31] T. L. Harris, "A pragmatic implementation of non-blocking linked-lists," In Proc. Int. Conf. Dist. Comput., London, UK, 2001, pp. 300-314.

[32] B. Hendrickson and R. Leland, The Chaco User's Guide Version 2. Sandia National Laboratories, Albuquerque NM, 1995.

[33] M. Herlihy, "A methodology for implementing highly concurrent data structures," In Proc. of the Second ACM SIGPLAN Symposium on Principles and Practice of Parallel Programming, Seattle, Washington, 1990, pp. 197-206.

[34] M. Herlihy, "A methodology for implementing highly concurrent data objects," ACM Trans. Prog. Lang. Sys., vol. 15, no. 5, pp. 745-770, 1993.

[35] M. Herlihy and N. Shavit, The Art of Multiprocessor Programming. San Francisco, CA: Morgan Kaufmann Publishers Inc., 2008.

[36] Intel. Reference Manual for Intel Threading Building Blocks, version 1.13. Intel Corp., Santa Clara, CA, 2009.

[37] Intel. Reference Manual for Intel Threading Building Blocks, version 1.0. Intel Corp., Santa Clara, CA, 2006. 
[38] J. JàJà, An Introduction Parallel Algorithms. Reading, MA: Addison-Wesley, 1992.

[39] E. Johnson, "Support for Parallel Generic Programming". PhD thesis, Indiana University, Indianapolis, 1998.

[40] E. Johnson and D. Gannon, "HPC++: Experiments with the parallel standard template library," In Proc. Int. Conf. on Supercomputing, Vienna, Austria, 1997, pp. 124-131.

[41] L. V. Kale and S. Krishnan, "CHARM++: A portable concurrent object oriented system based on C++," SIGPLAN Not., vol. 28, no. 10, pp. 91-108, 1993.

[42] G. Karypis and V. Kumar, "Multilevel k-way partitioning scheme for irregular graphs," J. of Parallel and Distributed Computing, vol. 48, no. 1, pp. 96-129, 1998.

[43] H. T. Kung and P. L. Lehman. "Concurrent manipulation of binary search trees," ACM Trans. Database Syst., vol. 5, no. 3, pp. 354-382, 1980.

[44] K. Mehlhorn and S. Naher, LEDA: A Platform for Combinatorial and Geometric Computing. New York: Cambridge University Press, 1999.

[45] M. D. Lam, E. E. Rothberg, and M. E. Wolf, "The cache performance and optimizations of blocked algorithms," In Proc. of the fourth Int. Conf. on Architectural Support for Programming Languages and Operating Systems, New York, NY, 1991, pp. 63-74.

[46] L. Lamport, "How to make a multiprocessor computer that correctly executes multiprocess programs," Computers, IEEE Transactions on, vol. C-28, no. 9, 
pp. 690 -691, Sep. 1979.

[47] P. L. Lehman and S. B. Yao. "Efficient locking for concurrent operations on b-trees," ACM Trans. Database Syst., vol. 6, no. 4, pp. 650-670, 1981.

[48] M. M. Michael, "High performance dynamic lock-free hash tables and list-based sets," In Proc. of the Fourteenth Annual ACM Symposium on Parallel Algorithms and Architectures, Winnipeg, Manitoba, Canada, 2002, pp. 73-82.

[49] D. Musser, G. Derge, and A. Saini, STL Tutorial and Reference Guide, Second Edition. Reading, MA: Addison-Wesley, 2001.

[50] J. Moreira, V. Salapura, G. Almasi, C. Archer, R. Bellofatto, P. Bergner, R. Bickford, M. Blumrich, J. Brunheroto, A. Bright, M. Brutman, J. Castanos, D. Chen, P. Coteus, P. Crumley, S. Ellis, T. Engelsiepen, A. Gara, M. Giampapa, T. Gooding, S. Hall, R. Haring, R. Haskin, P. Heidelberger, D. Hoenicke, T. Inglett, G. Kopcsay, D. Lieber, D. Limpert, P. McCarthy, M. Megerian, M. Mundy, M. Ohmacht, J. Parker, R. Rand, D. Reed, R. Sahoo, A. Sanomiya, R. Shok, B. Smith, G. Stewart, T. Takken, P. Vranas, B. Wallenfelt, M. Blocksome and J. Ratterman, "The Blue Gene/L supercomputer: A hardware and software story," International Journal of Parallel Programming, vol. 35, no. 3., pp. 181206.

[51] W. Pugh, "Concurrent maintenance of skip lists," Univ. of Maryland at College Park, Tech. Rep., UMIACS-TR-90-80, 1990.

[52] L. Rauchwerger, F. Arzu, and K. Ouchi "Standard templates adaptive parallel library (STAPL)," In Wkshp. on Lang. Comp. and Run-time Sys. for Scal. Comp., in Lecture Notes in Computer Science, vol. 1511, pp. 402-410, 1998. 
[53] J. W. Reynders, P. J. Hinker, J. C. Cummings, S. R. Atlas, S. Banerjee, W. F. Humphrey, S. R. Karmesin, K. Keahey, M. Srikant, and M. D. Tholburn, "POOMA: A framework for scientific simulations of paralllel architectures," In Gregory V. Wilson and Paul Lu, editors, Parallel Programming in $C++$ Cambridge, MA: MIT Press, 1996, pp. 547-588.

[54] S. Saunders and L. Rauchwerger, "Armi: An adaptive, platform independent communication library," In Proc. ACM SIGPLAN Symp. Prin. Prac. Par. Prog., San Diego, California, 2003, pp. 230-241.

[55] S. Saunders and L. Rauchwerger, "A parallel communication infrastructure for STAPL," In Wkshp. on Perf. Opt. for High-Level Languages and Libraries, New York, NY, Jun 2002.

[56] S. R. Alam, J. A. Kuehn, R. F. Barrett, J. M. Larkin, M. R. Fahey, R. Sankaran, P. H. Worley, "Cray XT4: An early evaluation for petascale scientific simulation," In Proc. of Supercomputing, Reno, NV, 2007, pp. 1-12

[57] N. Thomas, S. Saunders, T. Smith, G. Tanase, and L. Rauchwerger "Armi: A high level communication library for STAPL," Parallel Processing Letters, vol. 16, no. 2, pp. 261-280, 2006.

[58] S. Saunders, "Object Oriented Abstractions for Communication in Parallel Programs". M.S. thesis, Texas A\&M University, College Station, 2003.

[59] T. J. Sheffler, "The amelia vector template library," In G. V. Wilson and P. Lu, editors, Parallel Programming in $\mathrm{C}++$, Scientific and Engineering Computation Series, pages 43-90, Cambridge, MA: MIT Press, 1996. 
[60] J. Siek, L. Lee and A .Lumsdaine, The Boost Graph Library: User Guide and Reference Manual. Reading, MA: Addison-Wesley, 2001.

[61] J. Siek and A. Lumsdaine, "The matrix template library: Generic components for high-performance scientific computing," Computing in Science and Eng., vol. 1, no. 6, pp. 70-78, 1999.

[62] D. Skinner, "Performance monitoring of parallel scientific applications," National Energy Research Scientific Computing Center, Lawrence Berkeley National Laboratory, Berkeley, CA, Tech. Rep., LBNL-5503, 2005.

[63] G. Tanase, M. Bianco, N. M. Amato, and L. Rauchwerger, "The STAPL pArray," In Proc. of the 2007 Workshop on Memory Performance (MEDEA), Brasov, Romania, 2007, pp. 73-80.

[64] G. Tanase, C. Raman, M. Bianco, N. M. Amato, and L. Rauchwerger, "Associative parallel containers in STAPL," In Int. Workshop on Languages and Compilers for Parallel Computing, in Lecture Notes in Computer Science, vol. 5234, pp. 156-171, 2008.

[65] G. Tanase, X. Xu, A. Buss, Harshvardhan, I. Papadopoulos, O. Pearce, T. Smith, N. Thomas, M. Bianco, N. M. Amato, and L. Rauchwerger, "The STAPL pList," In Int. Workshop on Languages and Compilers for Parallel Computing, in Lecture Notes in Computer Science, vol. 5898, pp. 16-30, 2009.

[66] G. Tanase, A. Buss, A. Fidel, Harshvardhan, I. Papadopoulos, O. Pearce, T. Smith, N. Thomas, X. Xu, N. Mourad, J. Vu, M. Bianco, N. M. Amato, and L. Rauchwerger, "The STAPL pContainer Framework," In Proc. ACM SIGPLAN Symp. Prin. Prac. Par. Prog., San Antonio, TX, 2011, to appear. 
[67] N. Thomas, G. Tanase, O. Tkachyshyn, J. Perdue, N. M. Amato, and L. Rauchwerger, "A framework for adaptive algorithm selection in STAPL," In Proc. ACM SIGPLAN Symp. Prin. Prac. Par. Prog., Chicago, IL, 2005, pp. 277-288.

[68] S. Thomas, G. Tanase, L. K. Dale, J. M. Moreira, L. Rauchwerger, and N. M. Amato, "Parallel protein folding with STAPL," Concurrency and Computation: Practice and Experience, vol. 17, no. 14, pp. 1643-1656, 2005.

[69] J. D. Valois, "Lock-free linked lists using compare-and-swap," In Proc. ACM Symp. on Princ. of Dist. Proc. (PODC), New York, NY, 1995 , pp. 214-222.

[70] K. Yelick, L. Semenzato, G. Pike, C. Miyamoto, B. Liblit, A. Krishnamurthy, P. Hilfinger, S. Graham, D. Gay, P. Colella, and A. Aiken, "Titanium: A highperformance Java dialect," In ACM, editor, ACM 1998 Workshop on Java for High-Performance Network Computing, New York, NY, 1998. 


\section{VITA}

Gabriel Tanase received his Bachelor of Science from the Polytechnic University of Bucharest, Romania in May 1999. He graduated in the top 5\% of his class and his thesis was titled "Adaptive Parallelism using TupleSpace". In May, 2000 he received his Master of Science from the Polytechnic University of Bucharest, Romania. His Master's thesis was titled "Parallel Algorithms for STAPL".

Gabriel Tanase did his Ph.D. studies in the Department of Computer Science at Texas A\&M University working with Dr. Lawrence Rauchwerger and Dr. Nancy Amato in the Software \& Systems Group of the Parasol Lab. His research interests are in the area of high performance computing, including parallel programming languages and libraries, parallel algorithms and generic programming. He received his Ph.D. in computer science from Texas A\&M University in December 2010.

More information about Gabriel Tanase' research and publications may be found at http://parasol.tamu.edu/people/gabrielt. He may be reached at: Parasol Lab, 301 Harvey R. Bright Bldg, 3112 TAMU, College Station, TX 77843-3112.

The typist for this dissertation was Gabriel Tanase. 